\title{
DYNAMICS OF SINGULAR COMPLEX ANALYTIC VECTOR FIELDS WITH ESSENTIAL SINGULARITIES I
}

\author{
ALVARO ALVAREZ-PARRILLA AND JESÚS MUCIÑO-RAYMUNDO \\ Dedicated to Luz Ximena; she loves the pictures.
}

Abstract. We tackle the problem of understanding the geometry and dynamics of singular complex analytic vector fields $X$ with essential singularities on a Riemann surface $M$ (compact or not). Two basic techniques are used. (a) In the complex analytic category on $M$, we exploit the correspondence between singular vector fields $X$, differential forms $\omega_{X}$ (with $\omega_{X}(X) \equiv 1$ ), orientable quadratic differentials $\omega_{X} \otimes \omega_{X}$, global distinguished parameters $\Psi_{X}(z)=\int^{z} \omega_{X}$, and the Riemann surfaces $\mathcal{R}_{X}$ of the above parameters. (b) We use the fact that all singular complex analytic vector fields can be expressed as the global pullback via certain maps of the holomorphic vector fields on the Riemann sphere, in particular, via their respective $\Psi_{X}$.

We show that under certain analytical conditions on $\Psi_{X}$, the germ of a singular complex analytic vector field determines a decomposition in angular sectors; center $C$, hyperbolic $H$, elliptic $E$, parabolic $P$ sectors but with the addition of suitable copies of a new type of entire angular sector $\mathscr{E}$, stemming from $X(z)=\mathrm{e}^{z} \frac{\partial}{\partial z}$. This extends the classical theorems of A. A. Andronov et $a l$. on the decomposition in angular sectors of real analytic vector field germs.

The Poincaré-Hopf index theory for $\mathfrak{R e}(X)$ local and global on compact Riemann surfaces, is extended so as to include the case of suitable isolated essential singularities.

The inverse problem: determining which cyclic words $\mathcal{W}_{X}$, comprised of hyperbolic, elliptic, parabolic and entire angular sectors, it is possible to obtain from germs of singular analytic vector fields, is also answered in terms of local analytical invariants.

We also study the problem of when and how a germ of a singular complex analytic vector field having an essential singularity (not necessarily isolated) can be extended to a suitable compact Riemann surface.

Considering the family of entire vector fields $\mathcal{E}(d)=\left\{X(z)=\lambda \mathrm{e}^{P(z)} \frac{\partial}{\partial z}\right\}$ on the Riemann sphere, where $P(z)$ is a polynomial of degree $d$ and $\lambda \in \mathbb{C}^{*}$, we completely characterize the local and global dynamics of this class of vector fields, compute analytic normal forms for $d=1,2,3$, and show that for $d \geq 3$ there are an infinite number of topological (phase portrait) classes of $\mathfrak{R e}(X)$, for $X \in \mathcal{E}(d)$. These results are based on the work of R. Nevanlinna, A. Speisser and M. Taniguchi on entire functions $\Psi_{X}$.

Finally, on the topological decomposition of real vector fields into canonical regions, we extend the results of L. Markus and H. E. Benzinger to meromorphic on $\mathbb{C}$ vector fields $X$, with an essential singularity at $\infty \in \widehat{\mathbb{C}}$, whose $\Psi_{X}^{-1}$ have $d$ logarithmic branch points over $d$ finite asymptotic values and $d$ logarithmic branch points over $\infty$.

Received by the editors February 20, 2014 and, in revised form, July 8, 2016, July 29, 2016, December 14, 2016.

2010 Mathematics Subject Classification. Primary 32S65, Secondary 30F20, 58K45, 32M25.

Key words and phrases. Entire functions, entire vector fields, Riemann surfaces, essential singularities.

The first author was partially supported by UABC projects 1273 and 0196 .

The second author was partially supported by LAISLA. 


\section{Contents}

1. Introduction

2. The basic correspondences 134

2.1. Notation and conventions 134

2.2. Vector fields, differential forms, orientable quadratic differentials, flat metrics, distinguished parameters, Riemann surfaces

3. Vector fields under local and global maps

3.1. Pullback of vector fields by singular analytic maps

3.2. Every singular analytic vector field admits a global flow box and is a

\section{Newton vector field}

4. Vector field analytic invariants

4.1. Class, $\mathrm{p}$-order and type at an isolated essential singularity

4.2. Residues and semi-residues 144

4.3. Asymptotic values 145

5. Angular sectors at an isolated essential singularity 149

5.1. Angular sectors of vector fields in the real analytic category 149

5.2. Angular sectors $C, H, E, P, \mathscr{E}$ of vector fields in the complex analytic category

5.3. Attributes of the angular sectors.

5.4. Angular sectors on a Riemann surface

5.5. Angular sectors in the flat surfaces category 155

5.6. Isometric glueing

5.7. Cyclic words $\mathcal{W}_{X}$ at poles and zeros

5.8. Angular sectors of vector fields in the combinatorial category 163

5.9. Admissible words $\mathcal{W}$ at isolated essential singularities $\quad 165$

6. Poincaré-Hopf index theory

7. Extending vector field germs to compact Riemann surfaces

8. Entire structurally finite 1 -order $d$ vector fields $\mathcal{E}(d)$

8.1. Revisiting the Riemann surface $\mathcal{R}_{X}$ associated to $X \in \mathcal{E}(d)$

8.2. Why is the description of $\mathcal{R}_{X}$, for $X \in \mathcal{E}(d)$ difficult?

8.3. $d$-configuration trees

8.4. Natural equivalences in $\mathcal{E}(d)$

8.5. Description of the family $\mathcal{E}(d)$ via combinatorial scheme

8.6. Normal forms for $X \in \mathcal{E}(d)$, when $d=1,2,3$

8.7. The quotient space $\mathcal{E}(d) / A u t(\mathbb{C})$ and flat metrics

8.8. On the cardinality of the topological classes of $\mathfrak{R e}(X)$, for $X \in \mathcal{E}(d)$

9. From germs to admissible words and back

9.1. Admissible words from germs $((\mathbb{C}, 0), X)$

9.2. Germs from admissible words $\mathcal{W}_{X}$

10. A better vector field germ extension result

11. Appendix: Global decomposition into canonical regions, following L. Markus and H. E. Benzinger 


\section{INTRODUCTION}

We consider singular complex analytic vector fields $X$, having local expressions $\left\{f_{\mathrm{j}}(z) \frac{\partial}{\partial z}\right\}$ on a Riemann surface $M$ (compact or not), admitting a singular set $\mathcal{S}_{X}$. The singular set $\mathcal{S}_{X}$ consists of:

- zeros, poles, isolated essential singularities and

- accumulation points of the above kind of points on $M$, for the sake of simplicity we will call these essential singularities.

As brief terminology, analytic means complex analytic.

In order to describe the global structure of the real trajectory solutions of $X$, consider the following naive question:

How explicitly can we globally describe a singular analytic vector field $X$ on a Riemann surface $M$ ?

We use two main techniques. The first technique is the one-to-one correspondence on $M$ (respectively on $(\mathbb{C}, 0)$ in the case of germs) between:

1) Singular analytic vector fields $X$.

2) Singular analytic differential forms $\omega_{X}$, where $\omega_{X}(X) \equiv 1$.

3) Singular analytic orientable quadratic differentials $\omega_{X} \otimes \omega_{X}$.

4) Flat metrics $g_{X}$ associated to the quadratic differentials $\omega_{X} \otimes \omega_{X}$, with suitable singularities, provided with a real geodesic vector field.

5) Global singular analytic (additively automorphic, probably multivalued) distinguished parameters

$$
\Psi_{X}(z)=\int^{z} \omega_{X}
$$

6) The Riemann surfaces $\mathcal{R}_{X}$ associated to the maps $\Psi_{X}$.

Which enables us to transfer results from one area to the others; see Lemma 2.6 for the detailed correspondence statement.

As far as we know, unifying (1)-(5) came from the idea of (local) distinguished parameters of $\omega_{X} \otimes \omega_{X}$, see K. Strebel [66], pp. 20-21, where many of the geometric and dynamical aspects encoded in meromorphic quadratic differentials are described. The brothers Nevanlinna [53, pp. 298-303, A. Speiser 64 and M. Taniguchi 67, 68, have studied the relationship between (5) and (6) for entire and meromorphic functions. We look at the larger family of singular analytic objects, where the study of the global nature of (5) and (6) brings new insight into the description of vector fields.

The second technique is presented $₫ 3$. We follow Riemann's idea: "every compact hyperelliptic Riemann surface $M$ can be described as a ramified covering on the sphere $\widehat{\mathbb{C}}$, where the placings and orders of the ramification values determine $M$ "; see [51, Lecture I for this synthesis in the general case. Our assertion is:

"Every singular analytic vector field $X$ on $M$ (respectively on $(\mathbb{C}, 0)$ ) can be expressed as the pullback, via certain singular analytic probably multivalued maps $\Psi_{X}$ and $\Phi_{X}$, of the simplest analytic vector fields $\frac{\partial}{\partial t}$ or $-w \frac{\partial}{\partial w}$ on the Riemann sphere $\widehat{\mathbb{C}}$." 
As a concrete answer to the naive question, one has that the following commutative diagram holds true:

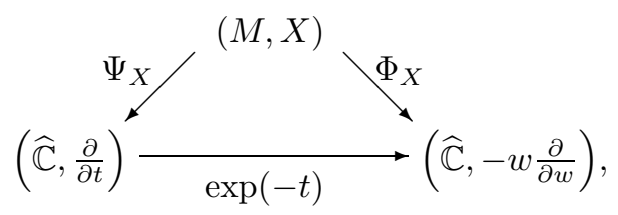

where $\Phi_{X}=\exp \circ\left(-\Psi_{X}\right)$; see $₫ 3.2$, in particular Remark 3.6 for the accurate statement of the diagram. In the language of differential equations:

- $X=\Psi_{X}^{*}\left(\frac{\partial}{\partial t}\right)$ means that $X$ has a global flow-box, i.e. the local rectificability can be analytically continued to $M$ minus the singular set $\mathcal{S}_{X}$.

- $X=\Phi_{X}^{*}\left(-w \frac{\partial}{\partial w}\right)$ states that $X$ is the global Newton vector field of $\Phi_{X}$. The usual interest stems from the fact that Newton vector fields $X$ have sinks exactly at the zeros of $\Phi_{X}$, and sources exactly at the poles of $\Phi_{X}$.

Even in the case of univalued maps, by Picard's Theorem, in the vicinity of an isolated essential singularity of $X$ the (a priori) local maps $\Psi_{X}$ and $\Phi_{X}$ cover $\widehat{\mathbb{C}}$ minus two points. Hence, the local complexity of $X$ at an isolated essential singularity on $(\mathbb{C}, 0)$ must be studied using the global maps $\Psi_{X}$ and $\Phi_{X}$. This can be readily seen in the example of $X(z)=\mathrm{e}^{z^{d}} \frac{\partial}{\partial z}$ where the essential singularity is at $\infty \in \widehat{\mathbb{C}}$.

Given a germ $((\mathbb{C}, 0), X)$ of a singular analytic vector field with an isolated essential singularity at 0 , roughly speaking, we have the following analytic invariants of $X$ :

- class $\mathrm{p}$, taking values in $\mathbb{N} \cup\{0, \infty\}$,

- $\mathrm{p}$-order, with values in $\mathbb{R} \cup\{\infty\}$,

- residue and semi-residues (of the respective $\omega_{X}$ ), taking values in $\mathbb{C}$,

- configurations of critical and asymptotic values (of the respective $\Psi_{X}$ ),

see $\$ 4$ and Corollary 4.18

We study local topological/analytical invariants of $((\mathbb{C}, 0), X)$ in $\$ 5$, We convene that the real trajectories of the associated real analytic vector field $\mathfrak{R e}(X)$ are to be called trajectories of the singular analytic vector field $X$ (the phase portraits of $X$ and $\mathfrak{R e}(X)$ coincide). Recall that a germ of a real analytic vector field $Z$ on $\left(\mathbb{R}^{2}, 0\right)$ having one isolated zero, admits a decomposition in angular sectors: hyperbolic, elliptic, and parabolic (see 55.1 ), thanks to a classical theorem of A. A. Andronov et al. (see [8, pp. 86, 37, pp. 144).

We propose complex analytic angular sectors; center $C$, hyperbolic $H$, elliptic $E$, parabolic $P$ and a new type of entire angular sector $\mathscr{E}$ (see Figure 3 ) based upon the entire vector field $X(z)=\mathrm{e}^{z} \frac{\partial}{\partial z}$ at the point $\infty \in \widehat{\mathbb{C}}$. Using these new sectors, a large family of germs of singular analytic vector fields $X$ on $(\mathbb{C}, 0)$, with 0 an isolated essential singularity, admit a similar decomposition. Roughly speaking, a germ $X$ determines an admissible word whenever it has an associated cyclic word $\mathcal{W}_{X}$ in the alphabet $C, H, E, P, \mathscr{E}$, arising from the topology of the trajectories of $X$ at the singularity, diagrammatically (see (5.15)):

$$
((\mathbb{C}, 0), X) \longmapsto \mathcal{W}_{X}=W_{1} W_{2} \cdots W_{k}, \quad W_{\iota} \in\{C, H, E, P, \mathscr{E}\}
$$


Essentially $\mathcal{W}_{X}$ is well defined modulo the relation $E \mathscr{E} H \sim \mathscr{E}$ (see Remark 5.18). The relation arises from to the ambiguity in the choice of representative for the germ of $X$.

As a concrete example, recall that $((\mathbb{C}, 0), X)$ having a pole, a simple zero with pure imaginary linear part or a multiple zero with zero residue determine the words

$$
\frac{1}{z^{k}} \frac{\partial}{\partial z} \longmapsto \underbrace{H \cdots H}_{2 k+2}, \quad i z \frac{\partial}{\partial z} \longmapsto C, \quad z^{s} \frac{\partial}{\partial z} \longmapsto \underbrace{E \cdots E}_{2 s-2} \quad s \geq 2 .
$$

An accurate description in the meromorphic case is in Table 2, §5. An example with an isolated essential singularity on $(\widehat{\mathbb{C}}, \infty)$ is

$$
\frac{\mathrm{e}^{z^{d}}}{d z^{d-1}} \frac{\partial}{\partial z} \longmapsto \underbrace{\mathscr{E} \cdots \mathscr{E}}_{2 d} \quad \text { for } d \geq 1
$$

see Example 5.15 , 2.

Let $h, e, p, \epsilon$ denote the number of hyperbolic $H$, elliptic $E$, parabolic $P$ and entire $\mathscr{E}$ angular sectors, respectively, in an admissible word $\mathcal{W}_{X}$. In 86 the Poincaré-Hopf index theory for $\mathfrak{R e}(X)$ is extended, starting from the classic formula of I. Bendixson, to vector fields with isolated essential singularities.

Theorem (A) (Local and global Poincaré-Hopf theory).

1) Let $((\mathbb{C}, 0), X)$ be a germ of a singular analytic vector field with an isolated singularity at 0 and further suppose that $X$ determines an admissible word $\mathcal{W}_{X}$. Then the Poincaré-Hopf index of $X$ at 0 is

$$
P H(X, 0)=1+\frac{e-h+\epsilon}{2} .
$$

2) Let $X$ be a singular analytic vector field on a compact $M$ having a discrete set of poles, zeros and isolated essential singularities determining admissible words at each singularity, then

$$
\chi(M)=\sum_{q \in M} P H(X, q) .
$$

Exploration of the conditions under which a germ of a singular analytic vector field can be extended to a compact Riemann surface yields the following result.

Theorem (B) (Extension of vector field germs to compact Riemann surfaces). Let $((\mathbb{C}, 0), X)$ be a germ of a singular analytic vector field having a nonnecessarily isolated singularity at 0 .

1) There exists an extended singular analytic vector field $\tilde{X}$ on a compact Riemann surface $M_{\mathfrak{g}}$, for each genus $\mathfrak{g} \geq 0$, such that:

the germ of $\widetilde{X}$ at some $p \in M$ is holomorphically equivalent to the germ $X$, with $\widetilde{X}$ having an additional finite number of zeros and poles.

2) If, in addition, $X$ determines an admissible word $\mathcal{W}_{X}$, then there exists an extended $\widetilde{X}$ on $\widehat{\mathbb{C}}$, having at most an additional pole and a finite number of simple zeros.

3) If, in addition, $X$ determines an admissible word $\mathcal{W}_{X}$, having Poincaré-Hopf index $P H(X, 0)=2-2 \mathfrak{g}$, for $\mathfrak{g} \in \mathbb{N} \cup\{0\}$, and residue Res $\left(\omega_{X}, 0\right)=0$, then there exists an extended $\widetilde{X}$ on a compact Riemann surface $M_{\mathfrak{g}}$, of genus $\mathfrak{g}$, having a) no other singularities on $M_{\mathfrak{g}}=\widehat{\mathbb{C}}$, when $\mathfrak{g}=0$, 
b) two new simple poles and no other singularities on $M_{\mathfrak{g}}$, when $\mathfrak{g} \geq 1$.

Where assertions (1), (2) and (3) correspond to Theorem [7.1, Corollary 7.3 and Corollary 10.3, respectively.

In 98 , we provide a complete answer to the problem, which was originally considered by K. Hockett and S. Ramamurti [34], of describing the families of vector fields on $\widehat{\mathbb{C}}$,

$$
\mathcal{E}(d)=\left\{X(z)=\lambda \mathrm{e}^{P(z)} \frac{\partial}{\partial z} \mid P(z) \text { a polynomial of degree } d \geq 1, \lambda \in \mathbb{C}^{*}\right\} .
$$

Explicit examples of $X \in \mathcal{E}(d)$ are presented in Figures 1, 3, 15, 16, and 19,

It is natural to hope that vector fields in the family $\mathcal{E}(d)$ should have a description as admissible words at $(\widehat{\mathbb{C}}, \infty)$. In order to answer this, and to also analytically classify the family $\mathcal{E}(d)$, in 8.3 , we introduce the main global combinatorial object: the $d$-configuration tree $\Lambda_{X}$ associated to $X \in \mathcal{E}(d)$.

Very roughly speaking, $d$-configuration trees are weighted trees with complex parameters as their weights; compare with [65] and [54. They provide an accurate description of the Riemann surface $\mathcal{R}_{X}$, as a ramified covering $\pi_{X, 2}: \mathcal{R}_{X} \rightarrow\left(\widehat{\mathbb{C}}, \frac{\partial}{\partial t}\right)$ (see diagram (2.6) ), by encoding the placement and ramification index of the ramification points of $\mathcal{R}_{X}$. The main results of this section are incorporated, without all the technical details; see the following.

Theorem (C) (Analytical and topological classification of $\mathcal{E}(d)$ ).

1) Singular analytic vector fields in $\mathcal{E}(d)$ are in one-to-one correspondence with classes of $d$-configuration trees, i.e.

$$
\mathcal{E}(d) \cong\left\{\left[\Lambda_{X}\right] \mid \Lambda_{X} \text { is a d-configuration tree }\right\} .
$$

2) The normal forms in $\mathcal{E}(d) / A u t(\mathbb{C})$, for $d \leq 3$, can be given as follows:

$$
\begin{array}{ccl}
\text { For } d=1, & e^{z} \frac{\partial}{\partial z} . & \\
\text { For } d=2, & \mu e^{z^{2}} \frac{\partial}{\partial z}, & \mu \in \mathbb{C}^{*} . \\
\text { For } d=3, \quad \mu e^{\left(-\frac{1}{3} z^{3}+p z\right)} \frac{\partial}{\partial z}, & \mu \in \mathbb{C}^{*}, p \in \mathbb{C},
\end{array}
$$

and in particular, the geometry of $\mathcal{E}(3) / A u t(\mathbb{C})$ is related to Airy's function.

3) For $d=1,2$, there are exactly d topological classes of $\mathfrak{R e}(X)$ for $X \in \mathcal{E}(d)$.

4) For each $d \geq 3$, there are an infinite number of topological classes of $\mathfrak{R e}(X)$ for $X \in \mathcal{E}(d)$.

5) $A \operatorname{germ}((\widehat{\mathbb{C}}, \infty), X)$ is the restriction of $X \in \mathcal{E}(d)$ if and only if $\infty \in \widehat{\mathbb{C}}$ is an isolated essential singularity and the admissible word $\mathcal{W}_{X}$ satisfies that

1) the residue of the word $\operatorname{Res}\left(\mathcal{W}_{X}\right)=0$,

2) the Poincaré-Hopf index of the word $P H\left(\mathcal{W}_{X}\right)=2$,

3) it has exactly $\epsilon=2 d$ class 1 entire sectors $\mathscr{E}$.

The correspondence of (1) through (5) and the text being: (1) with Theorem 8.16. (2) with Theorem 8.24 and 8.6.1, (3) and (4) with Theorem 8.31, and (5) with Corollary 10.1 
From Theorem (C.5) it follows that not all admissible words arise from singular analytic vector fields in $\mathcal{E}(d)$. Roughly speaking, the relationship between a germ of a singular analytic vector field and the admissible word is that: half the number $\epsilon$ of class 1 entire sectors $\mathscr{E}$ in the admissible word $\mathcal{W}_{X}$ corresponds to the 1 -order of the distinguished parameter $\Psi_{X}$. This is the contents of Theorems 9.1 and 9.8 .

Theorem (D) (Local realization and recognition of analytical invariants from admissible words).

1) A germ $((\mathbb{C}, 0), X)$ of a singular analytic vector field having an isolated essential singularity at 0 , and whose distinguished parameter $\Psi_{X}$ satisfies that $\Psi_{X}^{-1}$ has as unique singularities $d$ logarithmic branch points over $d$ finite asymptotic values $\left\{a_{j}\right\} \subset \mathbb{C}_{t}$ and $d$ logarithmic branch points over $\infty$, determines an admissible word $\mathcal{W}_{X}$ composed of sectors of type $H, E, P$ and $\mathscr{E}$. In particular, there are $\epsilon=2 d$ sectors of type $\mathscr{E}$.

2) Every admissible word $\mathcal{W}_{X}$ in the alphabet $C, H, E, P$, $\mathscr{E}$ comes from a germ $((\mathbb{C}, 0), X)$ of an isolated essential singularity of a complex analytic vector field.

For $\epsilon \geq 2$, the distinguished parameter $\Psi_{X}$ satisfies that $\Psi_{X}^{-1}$ has $\epsilon / 2$ logarithmic branch points over $\epsilon / 2$ finite asymptotic values and $\epsilon / 2$ logarithmic branch points over $\infty$.

In $\$ 11$ Appendix, we recall that the topological decomposition of the phase portrait for real vector fields into canonical regions (spiral, annulus and strips), essentially provided by L. Markus [47] for $C^{1}$ plane vector fields, has been enhanced by H. E. Benzinger [10] to include the rational category. We extend these results to the case of meromorphic vector fields on $\mathbb{C}$ with an essential singularity at $\infty \in \widehat{\mathbb{C}}$, whose $\Psi_{X}^{-1}$ have $d$ logarithmic branch points over $d$ finite asymptotic values and $d$ logarithmic branch points over $\infty$. See Corollary 11.3. The reader is encouraged to consult Table 3 in $₫ 11$, it provides a quick reference to the canonical regions used coherently throughout the work.

Naturally, the space of singular analytic vector fields, on $\widehat{\mathbb{C}}$, with an essential singularity is extremely complex. As an example of this complexity, we look at the class 2 vector field $X(z)=\mathrm{e}^{z} \mathrm{e}^{-\mathrm{e}^{z}} \frac{\partial}{\partial z}$ (see Figures 2 and 5) which also has an isolated essential singularity at $\infty \in \widehat{\mathbb{C}}$. With this example in mind, a new family of angular sectors can be constructed. The theory presented here is not enough to completely understand the class 2 case, except Theorem (B.1) which does apply and Theorem (A) which applies with minor modifications. This could be the subject of a further study.

The different categories in which we work: Our approach to singular analytic vector fields uses different techniques. As an attempt to clarify where things are going, let us recall that we are working in: the complex analytic category $(X$, $\left.\omega_{X}, \Psi_{X}\right)$; the flat singular surfaces category $\left(g_{X}\right.$ on $\left.M\right)$; the combinatorial and topological categories $\left(d-\right.$-configuration trees $\Lambda_{X}$, admissible words $\mathcal{W}_{X}$, topological phase portraits classes of $\mathfrak{R e}(X))$.

In particular, for the family $\mathcal{E}(d)$, diagram (1.2) shows some of the main relations between the combinatorial, analytical, topological and geometrical categories. Here, $\left\{d\right.$-configuration trees $\left.\left[\Lambda_{X}\right]\right\}$ means the space of classes of $d$-configuration trees; the normal forms are $\mathcal{E}(d) / A u t(\mathbb{C})$; the quotient $\mathcal{E}(d) / A u t(\mathbb{C}) \times S^{1}$ means the space of classes of flat metrics $\left\{g_{X}\right\}$ up to orientation preserving isometries; and the quotient $\mathcal{E}(d) / \mathrm{Homeo}(\mathbb{C})^{+}$means the space of phase portrait classes 
of $\mathfrak{R e}(X)$ up to orientation preserving homeomorphisms (see Definition [3.1]1).

$(1.2)$

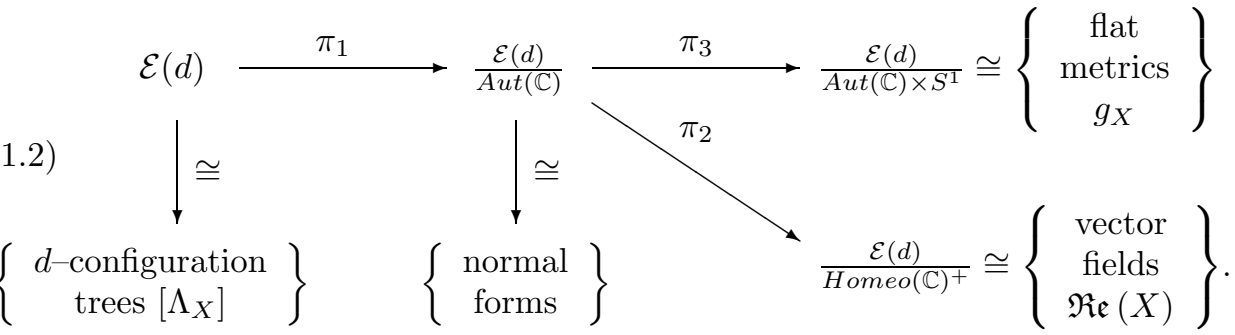

The following sources provided us enlightenment on many of the topics considered in the present work. We apologize in advance for our extremely brief and necessarily incomplete list.

As far as we are aware, the roots of the geometrical study of differential forms started in 1857 with B. Riemann: he distinguished differentials of the 1st kind (holomorphic), 2nd kind (meromorphic, zero residues) and 3rd kind (meromorphic, nonzero residues); see 58 pp. 96-97. Later on F. Klein implicitly described the geometrical behaviour of trajectories of $\omega_{X}$, see [43] pp. 1-9.

Modern treatises focusing on the trajectory structure of quadratic differentials: J. A. Jenkins [39, K. Strebel 66].

Transcendental singularities of meromorphic functions and Riemann surfaces: R. Nevanlinna [53, 54, M. Taniguchi 67], 68, W. Bergweiler et al. 12.

The point of view of differential equations (meromorphic vector fields): J. Gregor [26], 27], O. Hájek 31, 32, 33], N. A. Lukashevich [46, L. Brickman et al. 14], M. Sabatini 59, J. Muciño-Raymundo et al. 50, D. J. Needhan et al. 52, E. P. Volokitin et al. [70], A. Alvarez-Parrilla et al. [4, A. Garijo et al. 24], B. Branner et al. [13, E. Frías-Armenta et al. 23].

Partial versions of the correspondence (1)-(6): J. Muciño-Raymundo et al. [50], J. Muciño-Raymundo [49], A. Bustinduy et al. [17, [18.

Modern explicit proofs of the normal forms for zeros and poles of vector fields and quadratic differentials: J. A. Jenkins [39] ch. 3, J. Gregor [26, 27, L. V. Ahlfors [1 pp. 111, L. Brickman et al. 14], K. Strebel 66] ch. III, A. Garijo et al. 24].

The case of singularities for entire vector fields: K. Hockett et al. 34, A. Garijo et al. [25].

Relations with discrete dynamical systems: S. Smale [63], M. Shub et al. 61, H. E. Benzinger [10, H. T. Jonger et al. [40], A. Douady et al. 19], X. Buff et al. [16].

Flat metrics: K. Strebel [66, R. Peretz [57, J. Muciño-Raymundo et al. 50, H. Masur et al. [48, J. Muciño-Raymundo [49, M. Kontsevich et al. [44, J. P. Bowman et al. [15].

Graphs associated to global analytic functions: A. Speiser 65, R. Nevanlinna [54], G. Elfving [22, M. Shub et al. [61, H. T. Jongen et al. [40].

Acknowledgment. The authors are grateful to the referee for detailed comments and suggestions that greatly improved the exposition. 


\section{THE BASIC CORRESPONDENCES}

2.1. Notation and conventions. The material covered in this section can be found, for the meromorphic category, in [66, [50, 49]. Our present contribution is to consider essential singularities.

$\widehat{\mathbb{C}}=\mathbb{C} \cup\{\infty\}$ is the Riemann sphere.

$M$ denotes a connected Riemann surface, not necessarily compact.

Let $\left\{\phi_{\mathrm{j}}: V_{\mathrm{j}} \subset M \rightarrow \mathbb{C} \mid \mathrm{j} \in \mathcal{J}\right\}$ be a holomorphic atlas for $M$.

We use the canonical orientation of the Riemann surface, in particular, all maps between surfaces preserve orientation and paths that enclose points will have counterclockwise orientation.

Let $\mathcal{M}$ be a $C^{\infty}$ compact, oriented two manifold without boundary and let $q \in \mathcal{M}$ be a point. Consider a Riemann surface structure $M$ on $\mathcal{M} \backslash\{q\}$. Then, $q$ is a conformal puncture of $M$ when there exists a neighborhood $V \subset \mathcal{M}$ of $q$ and a biholomorphism $\varphi: V \backslash\{q\} \rightarrow\{0<|z|<1\} \subset \mathbb{C}$ such that $\lim _{p \rightarrow q} \varphi(p)=0$; or $a$ conformal hole when $\varphi: V \backslash\{q\} \rightarrow\{0<\varepsilon<|z|<1\}$.

Definition 2.1. A singular analytic vector field on $M$,

$$
X=\left\{f_{\mathrm{j}}(z) \frac{\partial}{\partial z} \mid z \in \phi_{\mathrm{j}}\left(V_{\mathrm{j}}\right)\right\},
$$

is a holomorphic vector field $X$ (on $M \backslash \mathcal{S}_{X}$ ), with singular set $\mathcal{S}=\mathcal{S}_{X}$ consisting of: zeros denoted by $\mathcal{Z}$; poles denoted by $\mathcal{P}$; isolated essential singularities denoted by $E$; and accumulation points in $M$ of zeros, poles and isolated essential singularities of $X$, so

$$
\mathcal{S}_{X}=\overline{(\mathcal{Z} \cup \mathcal{P} \cup E)} \subset M .
$$

Recall that $\overline{(~)}$ denotes the closure in $M$, and every $q \in \mathcal{S}_{X}$ determines a conformal puncture of $M$. We will define by

$$
\begin{array}{ll}
M^{\diamond}=M \backslash \bar{E}, & M^{0}=M \backslash \mathcal{S}_{X}, \\
M^{\prime}=M \backslash \overline{(\mathcal{Z} \cup E),} & M^{*}=M \backslash \overline{(\mathcal{P} \cup E)} .
\end{array}
$$

$(M, X)$ denotes a pair, Riemann surface and singular analytic vector field. $((M, q), X)$ denotes a germ of $X$ at $q \in M$.

Sometimes it will be convenient to consider germs at a conformal puncture, thus $((M \backslash\{q\}, q), X)$ denotes a germ of singular analytic vector field $X$ with $q \in M$ removed.

Remark 2.2. Due to the fact that the zeros of a vector field $X$ on $M$ are part of the singular set of $X$, we will require that an isolated essential singularity of $X$ be a point $q \in M$ where $X$ is holomorphic at a conformal punctured neighborhood $V \backslash\{q\}$ and $q$ is not an accumulation point of zeros.

Example 2.3. 1. Let $X(z)=\sin (1 / z) \frac{\partial}{\partial z}$ be a singular analytic vector field on $\widehat{\mathbb{C}}$. Then, $0 \in \widehat{\mathbb{C}}$ is not an isolated essential singularity of $X$, even though it is an isolated essential singularity of the function $f(z)=\sin (1 / z)$.

2. A natural example of a singular analytic vector field determining a conformal hole is

$$
X(z)=\left(\sum_{\nu=0}^{\infty} z^{2^{\nu}}\right) \frac{\partial}{\partial z} \quad \text { on } D(0,1)=\{|z|<1\} .
$$


The associated function $f(z)$ converges on $D(0,1)$ and diverges at the $2^{n}$-th roots of unity.

From the local coordinate expression in Definition 2.1. as usual the complex structure $J$ on $M$ is the automorphism of the real smooth tangent bundle $T_{\mathbb{R}} M$, locally given by

$$
J\left(\frac{\partial}{\partial x}\right)=\frac{\partial}{\partial y}, \quad J\left(\frac{\partial}{\partial y}\right)=-\frac{\partial}{\partial x} \quad \text { on } \quad \phi_{j}\left(V_{j}\right) .
$$

$T^{\prime} M$ denotes the holomorphic tangent line bundle of the Riemann surface $M$. If we provide $T_{\mathbb{R}} M$ with the complex structure from $J$, then we obtain an isomorphism of line bundles (having fibers $\mathbb{C}$ ) locally as

$$
\mathcal{I}: T_{\mathbb{R}} M \longrightarrow T^{\prime} M, \quad \frac{\partial}{\partial x} \longmapsto \frac{\partial}{\partial z} \quad \text { on } \quad \phi_{\mathrm{j}}\left(V_{\mathrm{j}}\right) ;
$$

see [42, ch. IX, 28, pp. 16-17, 71, pp. 70-72. Following the naming in [41, pp. 93 theorem 4.3, we have the real and imaginary parts of $X$ :

$$
\mathfrak{R e}(X):=u_{\mathfrak{j}}(x, y) \frac{\partial}{\partial x}+v_{\mathfrak{j}}(x, y) \frac{\partial}{\partial y}, \quad \mathfrak{I m}(X):=-v_{\mathrm{j}}(x, y) \frac{\partial}{\partial x}+u_{\mathrm{j}}(x, y) \frac{\partial}{\partial y} .
$$

Convention. By trajectories of a singular analytic vector field $X$, we understand the real maximal trajectory solutions

$$
\left\{z(\tau):\left(\tau_{\min }, \tau_{\max }\right) \subset \mathbb{R} \longrightarrow M^{0}\right\} \text { of } \dot{z}(\tau)=\mathfrak{R e}(X)(z(\tau)) .
$$

2.2. Vector fields, differential forms, orientable quadratic differentials, flat metrics, distinguished parameters, Riemann surfaces. Let $X=\left\{f_{\mathrm{j}}(z)\right.$ $\left.\frac{\partial}{\partial z}\right\}$ be a singular analytic vector field on $M$. The associated singular analytic differential form $\omega_{X}$ is such that $\omega_{X}(X) \equiv 1$. Furthermore, a geometrical interpretation is as follows. Slightly abusing notation 11 , for every simply connected $V_{\mathrm{j}} \subset M^{\prime}$,

$$
\Psi_{X, j}(z)=\int_{z_{0}}^{z} \omega_{X}: V_{\mathrm{j}} \subset M^{\prime} \longrightarrow \mathbb{C}_{t}
$$

sends small rectangles in $V_{\mathfrak{j}}$ whose sides are trajectories of $\mathfrak{R e}(X)$ and $\mathfrak{I m}(X)$, to euclidean rectangles in $\mathbb{C}_{t}$, whose sides are segments of horizontal and vertical lines, respectively. One also notices that on $V_{\mathrm{j}} \cap V_{\mathrm{k}}, \Psi_{X, \mathrm{j}}(z)=\Psi_{X, \mathrm{k}}(z)+a_{\mathrm{jk}}$ for some $a_{j \mathrm{k}} \in \mathbb{C}$.

A holomorphic analytic quadratic differential $\mathcal{Q}$ on $M$ is orientable if it is globally given as $\omega \otimes \omega$, for some singular analytic differential form $\omega$ on $M$. We say that $\mathcal{Q}_{X}=\omega_{X} \otimes \omega_{X}$ is a singular analytic orientable quadratic differential arising from $X$, and hence obtain a holomorphic atlas $\left\{\left(V_{j}, \Psi_{X, j}\right)\right\}$ for $M^{0}$ as above. The real foliation of $\frac{\partial}{\partial x}$ on $\mathbb{C}_{t}$ defines a horizontal foliation $\mathcal{F}_{\mathcal{Q}_{X}}$ on $M^{0}$, whose leaves correspond to the trajectories of $\mathfrak{R e}(X)$.

Let $g_{X}$ be the flat Riemannian metric on $M^{0}$ defined as the pullback under $\Psi_{X, j}$ of the usual flat metric on $\mathbb{C}_{t}$, i.e. $\Psi_{X, j}:\left(V_{j}, g_{X}\right) \rightarrow\left(\mathbb{C}_{t}, \delta\right)$ are isometries. The trajectories of $\mathfrak{R e}(X)$ and $\mathfrak{I m}(X)$ are orthonormal unitary geodesics in $\left(M^{0}, g_{X}\right)$. By abuse of language, we will say in many places that $\left(M^{0}, g_{X}\right)$ is induced by $\left(\widehat{\mathbb{C}}, \frac{\partial}{\partial t}\right)$ or $\left(\mathbb{C}, \frac{\partial}{\partial t}\right)$.

\footnotetext{
${ }^{1}$ Formally $z, z_{0} \in \phi_{\mathrm{j}}\left(V_{\mathrm{j}}\right)$ so we should be using $p=\phi_{\mathrm{j}}^{-1}(z)$ and $p_{0}=\phi_{\mathrm{j}}^{-1}\left(z_{0}\right)$ in (2.3). However, since in most cases $M=\widehat{\mathbb{C}}$ we use $z$ and $z_{0}$.
} 
In quadratic differentials theory, $\Psi_{X, j}$ is a distinguished parameter near a regular point for $\mathcal{Q}_{X}=\left\{d z^{2} /\left(f_{\mathrm{j}}(z)\right)^{2}\right\}$; see [66] pp. 20 .

It is to be noted that the distinguished parameter $\Psi_{X, j}$ has the following joyful properties: For $z_{1}$ and $z_{2}$ in a sufficiently small disk where $\omega_{X}$ is holomorphic,

$$
\Psi_{X, \mathrm{j}}\left(z_{2}\right)-\Psi_{X, \mathrm{j}}\left(z_{1}\right)=\int_{z_{1}}^{z_{2}} \omega_{X}=\left\{\begin{array}{l}
\text { complex time to travel from } z_{1} \text { to } z_{2} \\
\text { under the local holomorphic flow of } X .
\end{array}\right.
$$

Moreover, if $z_{1}$ and $z_{2}$ belong to the same real trajectory of $\mathfrak{R e}(X)$, then

$$
g_{X}-\text { length }\left(\overline{z_{1} z_{2}}\right)=\int_{\frac{z_{1} z_{2}}{}} \omega_{X}=\left\{\begin{array}{l}
\text { real time to travel from } z_{1} \text { to } z_{2} \\
\text { under the local real flow of } X
\end{array}\right.
$$

where $\overline{z_{1} z_{2}}$ means the geodesic segment in $\left(M^{0}, g_{X}\right)$.

A natural concept when considering multivalued functions is the following.

Definition 2.4. 1. A global analytic function $\Xi$ is a collection of function elements $\left\{\Xi_{j}: V_{j} \subset M \rightarrow \mathbb{C}_{t}\right\}$, which are related by direct analytic continuation (see [2] pp. 284).

2. Let $\Xi: M \rightarrow \mathbb{C}_{t}$ be a global analytic function with a nondense countable singular set $\mathcal{S}(\Xi) \subset M$, we say that $\Xi$ is additively automorphic (see [1], pp. 579) if given two branches one has $\Xi_{\mathrm{j}}(z)=\Xi_{\mathrm{k}}(z)+a_{\mathrm{jk}}$, for $a_{\mathrm{jk}} \in \mathbb{C}_{t}$.

Since we are interested in working with global analytic functions with singularities we shall emphasize this by using the term global singular analytic function (map) $\Xi$, i.e. the function elements $\left\{\left(V_{j}, \Xi_{j}\right)\right\}$ of $\Xi$ which are holomorphic, a priori do not determine an open cover of the whole $M$. When we need to emphasize the singular set of $\Xi$, we shall denote it by $\mathcal{S}(\Xi)$.

Definition 2.5. The global distinguished parameter of $X$ is the global singular analytic additively automorphic function $\Psi_{X}$, obtained from direct analytic continuation of a fixed function element $\Psi_{X, \iota}$ in $\left\{\Psi_{X, j} \mid j \in \mathcal{J}\right\}$, as in (2.3).

The relationship between $\mathcal{S}\left(\Psi_{X}\right)$, the singular set of the distinguished parameter, and $\mathcal{S}_{X}$, the singular set of the vector field $X$, will be discussed in $\$ 4.3$, Table 1 .

Moreover, the graph of $\Psi_{X}$,

$$
\mathcal{R}_{X}=\left\{(z, t) \mid t=\Psi_{X}(z)\right\} \subset M^{0} \times \widehat{\mathbb{C}}_{t},
$$

is the flat Riemann surface, obtained by analytic continuation. Note that the flat metric $\left(\mathcal{R}_{X}, \pi_{X, 2}^{*}\left(\frac{\partial}{\partial t}\right)\right)$ is induced by $\left(\widehat{\mathbb{C}}, \frac{\partial}{\partial t}\right)$ via the projection $\pi_{X, 2}$ in the following diagram:

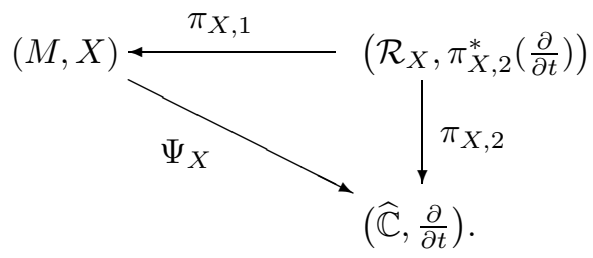

Summing up we get the following result (first introduced for (1-4) in [50, 49, for (5) in the rational case [17, [18 and now expanded to cover (6)). 
Lemma 2.6 (Basic correspondence). On any Riemann surface $M$ there is a canonical one-to-one correspondence between:

1) Singular analytic vector fields $X$, having singularities at $\mathcal{S}_{X}$.

2) Singular analytic differential forms $\omega_{X}$.

3) Singular analytic orientable quadratic differentials $\mathcal{Q}_{X}$.

4) Flat metrics $g_{X}$, with singularities on a nondense countable set $\mathcal{S} \subset M$, trivial holonomy in $M \backslash \mathcal{S}$ and a (real) geodesic unitary vector field $W_{X}$ with singularities exactly on $\mathcal{S}$. In fact, it must be true that $\mathcal{S} \subseteq \mathcal{S}_{X}$ and $W_{X}=\mathfrak{R e}(X)$.

5) Global singular analytic additively automorphic functions

$$
\Psi_{X}(z)=\int_{z_{0}}^{z} \omega_{X}: M^{\diamond} \longrightarrow \widehat{\mathbb{C}}_{t}
$$

where $z_{0} \in M^{\prime}$ and $z \in M^{\diamond}$.

6) The flat Riemann surface $\mathcal{R}_{X}$ associated to the global analytic additively automorphic function $\Psi_{X}$.

We will omit the sub-index $X$, when the context is clear.

Proof. $(1) \Leftrightarrow(2) \Leftrightarrow(3)$. The correspondence follows from

$$
f_{\mathrm{j}}(z) \frac{\partial}{\partial z} \longleftrightarrow \frac{d z}{f_{\mathrm{j}}(z)} \longleftrightarrow \frac{d z^{2}}{f_{\mathrm{j}}^{2}(z)} \quad z \in \phi_{\mathrm{j}}\left(V_{\mathrm{j}}\right) \text { for } V_{\mathrm{j}} \subset M \backslash \mathcal{S}_{X} ;
$$

see [50] and 49]. The above determine holomorphic sections, on $M \backslash \mathcal{S}_{X}$ : of the holomorphic tangent line bundle $T^{\prime} M$ ([28, pp. 16); the canonical line bundle $K_{M}$ ([28], pp. 146); and the line bundle of quadratic differentials $K_{M} \otimes K_{M}$, respectively.

$(2) \Leftrightarrow(5)$. Follows directly from the fundamental theorem of calculus: If $\Psi_{X}(z)=$ $\int_{z_{0}}^{z} \omega_{X}$ is a singular analytic additively automorphic function on $M^{\diamond}$, then $\omega_{X}$ is holomorphic on $M^{*}$. However, for $\Psi$ not additively automorphic the pullback of $\frac{\partial}{\partial t}$ via $\Psi$ will not result in a well-defined vector field (and thus $\omega=d \Psi$ will not be a well-defined differential form).

Note that $\Psi_{X}$ is well defined on $M^{\diamond}$. Furthermore, a pole $z_{0}$ of $X$ determines a holomorphic critical point of $\Psi_{X}$.

$(1) \Rightarrow(4)$. Let $X$ be a holomorphic section of $T^{\prime}\left(M \backslash \mathcal{S}_{X}\right)$ the holomorphic tangent line bundle of $M$ on the punctured $M \backslash \mathcal{S}_{X}$. Using $\mathcal{I}^{-1}$ in (2.1), we recover the real vector field $W_{X}:=\mathfrak{R e}(X)$ as a section of the real smooth tangent bundle $T_{\mathbb{R}}\left(M \backslash \mathcal{S}_{X}\right) . W_{X}$ is a unitary geodesic vector field for the metric $g_{X}$.

$(4) \Rightarrow(1)$. We can reformulate the initial flat surface data in (4) as $\left(\mathcal{M}_{0} \backslash \mathcal{S}_{0}, g_{0}, W_{0}\right)$ where $\mathcal{M}_{0}$ is a smooth oriented surface, $\mathcal{S}_{0} \subset M_{0}$ is a numerable set such that at each $p \in \mathcal{S}_{0}, \mathcal{M}_{0}$ is locally diffeomorphic to $\mathbb{R}^{2} \backslash\{\overline{0}\}, g_{0}$ is a smooth flat metric on $\mathcal{M}_{0} \backslash \mathcal{S}_{0}$, and $W_{0}$ is a unitary geodesic vector field on $\mathcal{M}_{0} \backslash \mathcal{S}_{0}$.

Since the flat metric has trivial holonomy and $\mathcal{M}_{0}$ is oriented, there exists a well-defined counterclockwise $\pi / 2$ rotation map, say

$$
J_{p}: T_{\mathbb{R}, p} \mathcal{M}_{0} \longrightarrow T_{\mathbb{R}, p} \mathcal{M}_{0}, \quad p \in \mathcal{M}_{0} \backslash \mathcal{S}_{0},
$$

on the real smooth tangent bundle of $T_{\mathbb{R}} \mathcal{M}_{0}$, satisfying $J_{p} \circ J_{p}=-I d$. Using this smooth complex structure, we have that $\left(\mathcal{M}_{0} \backslash \mathcal{S}_{0}, J\right)$ is a Riemann surface.

Moreover, $J$ allows us to recognize the points in $\mathcal{S}_{0}=\mathcal{S} \cup \mathcal{H} \subset \mathcal{M}_{0}$, where $\mathcal{S}$ denotes the conformal punctures and $\mathcal{H}$ the conformal holes. 
We recover the Riemann surface $M$ in (1), by defining

$$
M=\left(\mathcal{M}_{0} \cup \mathcal{S}, J\right) ;
$$

thus we disregard $\mathcal{H}$. In particular, if $\mathcal{H} \neq \varnothing$, then $M$ is necessarily noncompact. In order to obtain a singular analytic vector field, recall (2.1),

$$
\mathcal{I}: T_{\mathbb{R}}\left(\mathcal{M}_{0} \cup \mathcal{S}\right) \longrightarrow T^{\prime} M
$$

is a $\mathbb{C}$-linear isomorphism, 71 pp. 70-72. That is, in local coordinates the vector field

$$
W_{\mathrm{j}}=u_{\mathrm{j}}(x, y) \frac{\partial}{\partial x}+v_{\mathrm{j}}(x, y) \frac{\partial}{\partial y} \quad z \in \phi_{\mathrm{j}}\left(V_{\mathrm{j}}\right) \text { for } V_{\mathrm{j}} \subset \mathcal{M}_{0} \backslash \mathcal{S},
$$

considered as a smooth section of the real tangent bundle $T_{\mathbb{R}} \mathcal{M}_{0}$, corresponds to

$$
X_{\mathrm{j}}=f_{\mathrm{j}}(z) \frac{\partial}{\partial z}=\left(u_{\mathrm{j}}(x, y)+\sqrt{-1} v_{\mathrm{j}}(x, y)\right)\left(\frac{1}{2}\left(\frac{\partial}{\partial x}-\sqrt{-1} \frac{\partial}{\partial y}\right)\right) .
$$

Hence, $X_{\mathrm{j}}$ is a holomorphic section of the holomorphic tangent line bundle $T^{\prime}(M \backslash \mathcal{S})$; see [28, pp. 17, 42, pp. 129, and proposition 2.11.

Finally let $\mathcal{S}_{X}=\mathcal{S}$ and $W_{X}=\mathfrak{R e}(X)=W_{0}$.

$(5) \Leftrightarrow(6)$. Follows from the usual construction ([2 pp. 288) via direct analytic continuation of $\Psi_{X}$ and diagram (2.6).

A key observation that follows directly from diagram $(2.6)$ is:

Lemma 2.7. The following assertions are equivalent for $X$ a singular analytic vector field.

1) $\pi_{X, 1}$ is a biholomorphism between $\left(\mathcal{R}_{X}, \pi_{X, 2}^{*}\left(\frac{\partial}{\partial t}\right)\right)$ and $\left(M^{0}, X\right)$.

2) $\Psi_{X}: M^{\diamond} \rightarrow \widehat{\mathbb{C}}_{t}$ is univalued.

3) The residues and periods of $\omega_{X}$ are zero, i.e.

$$
\int_{\gamma} \omega_{X}=0 \quad \text { for every }[\gamma] \in H_{1}\left(M^{\prime}, \mathbb{Z}\right)
$$

\section{VECTOR FIELDS UNDER LOCAL AND GLOBAL MAPS}

\subsection{Pullback of vector fields by singular analytic maps.}

Definition 3.1. Let $X(z)=f(z) \frac{\partial}{\partial z}$ and $Y(z)=g(z) \frac{\partial}{\partial z}$ be two germs of singular analytic vector fields on $(\mathbb{C}, 0)$, let $\varphi_{f}(t, z)$ and $\varphi_{g}(t, z)$ be their local holomorphic flows, $t \in \mathbb{C}$.

1. $X$ and $Y$ are topologically equivalent (i.e. have topologically equivalent phase portraits) if there exists an orientation preserving homeomorphism $\Upsilon:(\mathbb{C}, 0) \rightarrow$ $(\mathbb{C}, 0)$ which takes trajectories of $\mathfrak{R e}(X)$ to trajectories of $\mathfrak{R e}(Y)$ preserving their orientation but not necessarily the real time parametrization.

2. $X$ and $Y$ are holomorphically equivalent if in addition $\Upsilon$ is a biholomorphism such that

$$
\Upsilon\left(\varphi_{f}(t, z)\right)=\varphi_{g}(t, \Upsilon(z))
$$

whenever both sides are well defined.

The global concepts (admitting singularities as in definition 2.1) are analogous.

Under the assumption that $\Upsilon$ is a biholomorphism, (3.1) is equivalent to $X=\Upsilon^{*} Y$. 
Remark 3.2. On Riemann surfaces, the following assertions are equivalent:

1. The singular analytic vector fields $(M, X)$ and $(N, Y)$ are holomorphically equivalent via a biholomorphism $\Upsilon: M \rightarrow N$.

2. There is an orientation preserving isometry $\Upsilon:\left(M^{0}, g_{X}\right) \rightarrow\left(N^{0}, g_{Y}\right)$ that takes geodesic trajectories of $\mathfrak{R e}(X)$ to geodesic trajectories of $\mathfrak{R e}(Y)$ preserving their real time orientation.

Lemma 3.3. Given a singular analytic vector field $Y=\left\{g_{\mathrm{k}}(t) \frac{\partial}{\partial t}\right\}$ on $N$ and a nonconstant, univalued, singular analytic map $\Upsilon: M \rightarrow N$, the pullback vector field $X=\Upsilon^{*} Y$ is a singular analytic vector field well defined on $M$. In particular,

$$
f_{\mathrm{j}}\left(z_{\mathrm{j}}\right)=\frac{g_{\mathrm{k}}\left(\Upsilon_{\mathrm{jk}}\left(z_{\mathrm{j}}\right)\right)}{\Upsilon_{\mathrm{jk}}^{\prime}\left(z_{\mathrm{j}}\right)}
$$

where $\Upsilon_{j \mathrm{k}}=\phi_{\mathrm{k}} \circ \Upsilon \circ \phi_{\mathrm{j}}^{-1}, \Upsilon_{j \mathrm{k}}^{\prime}=\frac{d \Upsilon_{\mathrm{jk}}}{d z}$, and $\left\{\phi_{\mathrm{j}}: V_{\mathrm{j}} \subset M \rightarrow \mathbb{C}\right\},\left\{\phi_{\mathrm{k}}: U_{\mathrm{k}} \subset N \rightarrow \mathbb{C}\right\}$ are charts of $M$ and $N$, respectively.

Example 3.4. Holomorphic vector fields on the Riemann sphere. The holomorphic vector fields on $\widehat{\mathbb{C}}$ are isomorphic to the Lie algebra $\mathfrak{p s l}(2, \mathbb{C})$. According to diagram (1.1), up to pullback by $T(z) \in P S L(2, \mathbb{C})$, we get two nontrivial families.

1. $Y(t)=\frac{\partial}{\partial t}$, hence $\left(\mathbb{C}, g_{Y}\right)$ is isometric to the euclidean plane, provided with the geodesic vector field $\frac{\partial}{\partial x}$.

2. $Y(w)=\lambda w \frac{\partial}{\partial w}, \lambda \in \mathbb{C}^{*}$, hence $\mathfrak{R e}(Y)$ has two centers if $\mathfrak{R e}(\lambda)=0$; one source and one sink otherwise. $\left(\mathbb{C}^{*}, g_{Y}\right)$ is isometric to a euclidean cylinder $S_{\rho}^{1} \times \mathbb{R}$, provided with the geodesic vector field, where $\rho$ is the perimeter which will be explicitly calculated in $\$ 5.7$

3.2. Every singular analytic vector field admits a global flow box and is a Newton vector field.

Proposition 3.5. Let $X$ be a singular analytic vector field on $M$, the following relations are fulfilled.

1) $X=\Psi^{*}\left(\frac{\partial}{\partial t}\right)$ for a (possibly multivalued) global singular analytic map $\Psi$.

2) $X=\Phi^{*}\left(-w \frac{\partial}{\partial w}\right)$ for a (possibly multivalued) global singular analytic map $\Phi$.

Furthermore, $\frac{\partial}{\partial t}$ is the pullback via $\exp (-t)$ of $-w \frac{\partial}{\partial w}$ and we have the commutative diagram (1.1).

Proof. For (1) consider the singular analytic map

$$
\Psi(z)=\int_{z_{0}}^{z} \omega_{X}: M^{\diamond} \longrightarrow \widehat{\mathbb{C}}_{t}, \quad z_{0} \in M^{\prime},
$$

applying Lemma 3.3 for $\left(\widehat{\mathbb{C}}, \frac{\partial}{\partial t}\right)$. Moreover, $\Psi$ is univalued if and only if the periods and residues of $\omega_{X}$ are zero; see Lemma 2.7

Analogously for assertion (2) use Lemma 3.3 and the singular analytic map

$$
\Phi(z)=\exp \left(-\int_{z_{0}}^{z} \omega_{X}\right): M^{\diamond} \backslash \Psi^{-1}(\infty) \longrightarrow \widehat{\mathbb{C}}_{w}, \quad z_{0} \in M^{\prime},
$$

for $\left(\widehat{\mathbb{C}},-w \frac{\partial}{\partial w}\right) . \Phi$ is univalued if and only if the periods and residues of $\omega_{X}$ are integer multiples of a suitable $\Pi \in \mathbb{C}^{*}$, i.e.

$$
n \Pi=\int_{\gamma} \omega_{X} \quad \text { for every }[\gamma] \in H_{1}\left(M^{\prime}, \mathbb{Z}\right) \text { and } n \in \mathbb{Z} .
$$


Remark 3.6. We abuse notation in diagram (1.1) by saying that:

1. The domain of $\Psi$ is $M$, when in reality it should be $M^{\diamond}$ for $\Psi$.

2. The domain of $\exp (-t)$ is $\widehat{\mathbb{C}}_{t}$, when in reality it should be $\mathbb{C}_{t}$.

3. The domain of $\Phi$ is $M$, when in reality it should be $M^{\diamond} \backslash \Psi^{-1}(\infty)$ for $\Phi$.

Thus, strictly speaking, the diagram (1.1) commutes whenever the analytic continuation of the maps $\Psi$ and $\Phi$ is well defined.

Remark 3.7. The vector field $X$ obtained as a pullback via $\Phi$ of $-w \frac{\partial}{\partial w}$ is known as the Newton vector field of the map $\Phi$. These have been extensively studied in several contexts; see 62, 63.

In particular, following H. E. Benzinger [10, who did it for the rational case, we extend his results to the singular analytic category and show that the function $\Phi$ provides a scheme for visualizing singular analytic vector fields $X$. Following Smale's original idea, along a trajectory $z(\tau)$ of $X$ one has that

$$
\Phi(z(\tau))=\Phi\left(z_{0}\right) \mathrm{e}^{-\tau}, \quad \text { for all } \tau \in \mathbb{R},
$$

hence $h(z)=\arg (\Phi(z))$ is a first integral of $\mathfrak{R e}(X)$. This technique is used to visualize the examples given in this paper (see [3, 4], [5] for further details).

Example 3.8. On the torus $M=\mathbb{C} /(\mathbb{Z} \oplus \tau \mathbb{Z})$, where as is usual $\mathfrak{I m}(\tau)>0$, every elliptic function $f(z)$ determines a meromorphic vector field $X(z)=f(z) \frac{\partial}{\partial z}$. Moreover, $X$ is a Newton vector field; see [4].

Example 3.9 (The exponential vector field on $\widehat{\mathbb{C}}$ ). The entire vector field with an isolated essential singularity at $\infty \in \widehat{\mathbb{C}}$,

$$
X(z)=\mathrm{e}^{z} \frac{\partial}{\partial z}
$$

is obtained as the pullback via $\Psi(z)=-\mathrm{e}^{-z}$ from the vector field $\frac{\partial}{\partial t}$. Note that $\Psi$ determines an infinitely ramified cover of $\widehat{\mathbb{C}}_{t}$ with the minimal number of ramification values, hence let us recognize $X$ as the simplest vector field having an isolated essential singularity. On $\mathbb{C}_{z}, \mathfrak{R e}(X)$ determines a two-dimensional Reeb component on each strip $\{2 \pi k \leq \mathfrak{I m}(z) \leq 2 \pi(k+1)\}$ for $k \in \mathbb{Z}$. See Figure 1
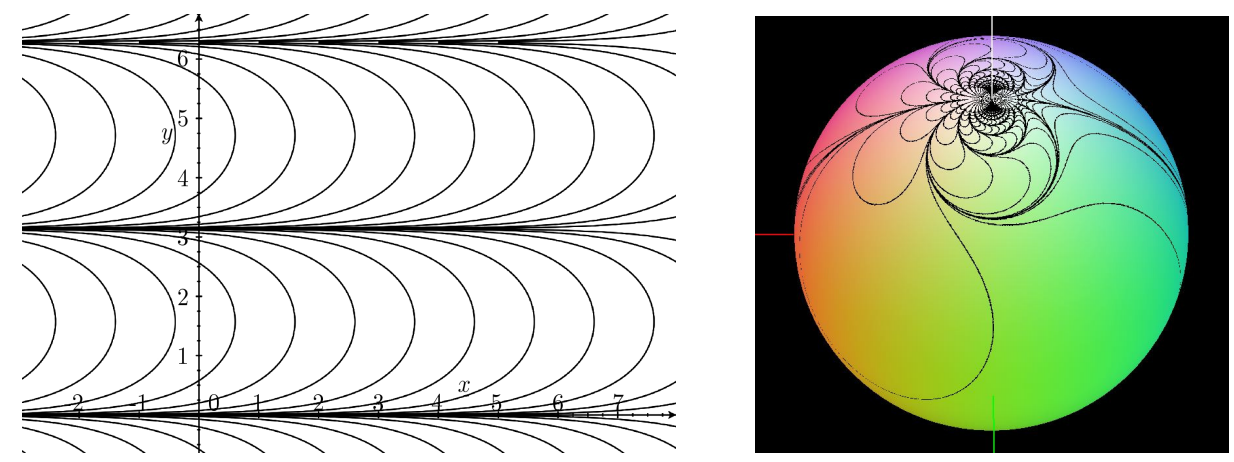

Figure 1. Phase portrait of $X(z)=\mathrm{e}^{z} \frac{\partial}{\partial z}$, (a) in the vicinity of the origin, (b) in the vicinity of $\infty \in \widehat{\mathbb{C}}$. See Example 3.9. 
Example 3.10 (Class 2 essential singularity 2 on $\widehat{\mathbb{C}}$ ). The entire vector field

$$
X(z)=\mathrm{e}^{z} \mathrm{e}^{-\mathrm{e}^{z}} \frac{\partial}{\partial z}
$$

is the pullback via $\Psi(z)=\mathrm{e}^{-\mathrm{e}^{z}}$ of $\frac{\partial}{\partial t}$. It has an isolated essential singularity at $\infty \in \widehat{\mathbb{C}}$. See Figure 2 .

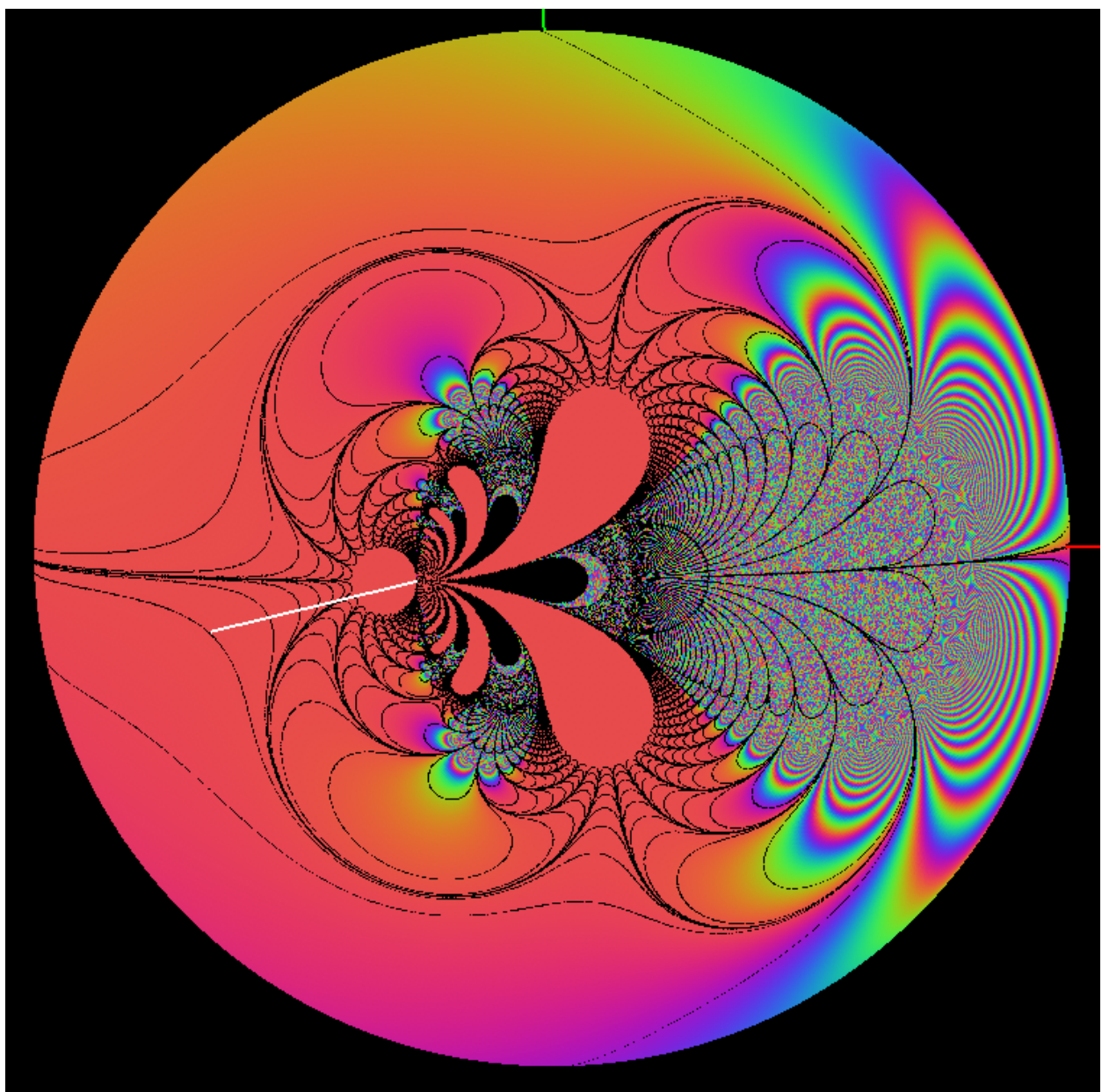

Figure 2. Phase portrait of $X(z)=\mathrm{e}^{z} \mathrm{e}^{-\mathrm{e}^{z}} \frac{\partial}{\partial z}$ in the vicinity of $\infty \in \widehat{\mathbb{C}}$. See Examples 3.10 and 5.9 .

${ }^{2}$ We define the class $\mathrm{p}$ of a vector field in 4.1 see also Example 4.6 


\section{VECTOR FIELD ANALYTIC INVARIANTS}

4.1. Class, $\mathrm{p}$-order and type at an isolated essential singularity. According to the basic correspondence (Lemma 2.6), singular analytic vector fields are related to global singular analytic additively automorphic functions of the form $\left\{\Psi_{X, j}=\right.$ $\left.\int\left(d z / f_{\mathrm{j}}\right)\right\}$, hence it will be convenient to examine, and extend to vector fields, certain analytic invariants of functions.

In the usual literature, the class, order and type (of growth) is defined for entire functions; see [11, pp. 352, [53, ch. 8, 60, ch. 3. As far as we know, A. Garijo et al. in [25], were the first to use the order of growth for vector fields.

Let $\psi:(\mathbb{C} \backslash\{0\}, 0) \rightarrow \mathbb{C}$ be a germ of a complex analytic function with an isolated singularity at $z=0$; i.e. $\psi$ has a pole, or an isolated essential singularity at the origin. We can define for $\varepsilon>0$,

$$
M_{\varepsilon}(\psi)=\max _{|z|=\varepsilon}\{\log |\psi(z)|\}
$$

and

$$
\log _{\mathrm{p}}(x)= \begin{cases}x, & \text { for } \mathrm{p}=0, \\ \underbrace{\log (\log (\cdots \log (x) \cdots))}_{\mathrm{p} \text {-times }}, & \text { for } \mathrm{p} \in \mathbb{N} .\end{cases}
$$

Let $\psi$ be as above, we say that:

1) $\psi$ is of class zero (at 0 ) if

$$
\limsup _{\varepsilon \rightarrow 0} \frac{M_{\varepsilon}(\psi)}{-\log (\varepsilon)}<\infty
$$

(note the choice $-\log$, since $\varepsilon \rightarrow 0$ ).

2) $\psi$ is of finite class $\mathrm{p} \in \mathbb{N}$ (at 0 ), denoted by $\mathrm{p}$-class, if $\mathrm{p}$ is the smallest natural such that

$$
\limsup _{\varepsilon \rightarrow 0} \frac{\log _{\mathrm{p}}\left(M_{\varepsilon}(\psi)\right)}{-\log (\varepsilon)}<\infty .
$$

3) $\psi$ is of infinite class (at 0 ) if no such natural number p exists.

4) For $\psi$ of class $\mathrm{p} \in \mathbb{N} \cup\{0\}$, the number $\rho_{\mathrm{p}} \in \mathbb{R}$ determined by

$$
\rho_{\mathrm{p}}(\psi)=\limsup _{\varepsilon \rightarrow 0} \frac{\log _{\mathrm{p}}\left(M_{\varepsilon}(\psi)\right)}{-\log (\varepsilon)}
$$

is called the $\mathrm{p}$-order of growth of $\psi$ (at 0 ) when $\rho_{\mathrm{p}}(\psi) \geq 0$ or the $\mathrm{p}$-order of vanishing of $\psi$ (at 0 ) when $\rho_{\mathrm{p}}(\psi)<0$.

5) For functions of class $\mathrm{p}$ and nonzero $\mathrm{p}$-order of growth $\rho_{\mathrm{p}} \neq 0$, we also have the concept of $\mathrm{p}$-type $\tau_{\mathrm{p}}$ defined as

$$
\tau_{\mathrm{p}}(\psi)=\limsup _{\varepsilon \rightarrow 0} \frac{\log _{\mathrm{p}-1}\left(M_{\varepsilon}(\psi)\right)}{\varepsilon^{-\rho_{\mathrm{p}}}} .
$$

For convenience of notation we shall allow $\rho_{\mathrm{p}}$ to be $\pm \infty$ when needed. We can now extend the above concepts to germs of vector fields and differential forms.

Definition 4.1. Let $\left((\mathbb{C}, 0), X(z)=f(z) \frac{\partial}{\partial z}\right)$ be a germ of a singular analytic vector field, with 0 an isolated singularity of $X$. We say that:

1. $X$ is of class zero if $f$ is of class zero.

2. $X$ is of class $\mathrm{p}$ if $f$ is of class $\mathrm{p}$.

3. $X$ is of infinite class if $f$ is of infinite class. 
4. For vector fields $X$ of class $\mathrm{p}$, the $\mathrm{p}$-order of $X$ is the corresponding for $f$, i.e. $\rho_{\mathrm{p}}(X):=\rho_{\mathrm{p}}(f)$.

5. Similarly, for the $\mathrm{p}$-type of $X$ we have $\tau_{\mathrm{p}}(X):=\tau_{\mathrm{p}}(f)$.

Analogously, if $\omega_{X}(z)=d z / f(z)$ is a germ of a differential form with 0 an isolated singularity of $\omega_{X}$, then $\omega_{X}$ inherits the class, order and type from that of the function $1 / f$.

Remark 4.2. 1. The definitions of class, order and type of $X$ make sense even in the case when 0 is an accumulation point of zeros for a vector field, respectively, an accumulation point of poles for the differential form $\omega_{X}$.

2. These definitions are invariant under conformal change of coordinates so they make sense for $X$ on a Riemann surface.

Example 4.3. Let $X(z)=z^{n} g(z) \frac{\partial}{\partial z}$ be a germ on $(\mathbb{C}, 0)$, with $g$ holomorphic at 0 such that $g(0) \neq 0$ and $n \in \mathbb{Z}$, then $X(z)$ is of class 0 and

$$
\rho_{0}(X)=-n,
$$

i.e. for finite class zero, the 0 -order corresponds to the negative of the usua 3 order of a zero or pole.

For $X$ an entire vector field, on $\mathbb{C}$, the interesting growth is at infinity. From the above definitions we recover (through the change of variable $z \mapsto 1 / z$ for $\widehat{\mathbb{C}}$ ), the usual theory for entire functions and growth at $\infty \in \widehat{\mathbb{C}}$. For example, let $f$ be an entire function, the respective $X(z)=f(z) \frac{\partial}{\partial z}$ is of finite class $\mathrm{p} \in \mathbb{N}$ at $\infty \in \widehat{\mathbb{C}}$, if $\mathrm{p}$ is the smallest natural number such that

$$
\limsup _{\varepsilon \rightarrow \infty} \frac{\log _{\mathrm{p}}\left(M_{\varepsilon}(f)\right)}{\log (\varepsilon)}<\infty .
$$

Example 4.4. Let $X(z)=\mathrm{e}^{P(z)} \frac{\partial}{\partial z}$, where $P(z)$ is a polynomial of degree $d$. Then at $\infty \in \widehat{\mathbb{C}}, X$ is of class 1 and its 1 -order $\rho_{1}(X)=d$. We will further study the family comprised of these vector fields in 8 .

Example 4.5. Consider the entire vector field of Example 3.10, $X(z)=\mathrm{e}^{z} \mathrm{e}^{-\mathrm{e}^{z}} \frac{\partial}{\partial z}$ at $\infty \in \widehat{\mathbb{C}}$, it is of class 2 and its 2 -order of growth is

$$
\rho_{2}(X)=\limsup _{\varepsilon \rightarrow 0} \frac{\log _{2}\left(M_{\varepsilon}(f)\right)}{\log (\varepsilon)}=1 .
$$

Example 4.6. If

$$
X(z)=\underbrace{\exp (\exp (\cdots \exp (g(z)) \cdots))}_{\text {p-times }} \frac{\partial}{\partial z},
$$

for $g$ an entire function of class zero, then the respective $X(z)$ is of $\mathrm{p}$-class at $z=\infty$. We have that

$$
\rho_{\mathrm{p}}(X)=\rho_{0}(g) \text {. }
$$

In the global case $M=\widehat{\mathbb{C}}$, for entire vector fields, without zeros, the comparison between $X$, and the respective $\omega_{X}$ and $\Psi_{X}$ is simple:

\footnotetext{
${ }^{3}$ In the sense of L. V. Ahlfors 2 pp. 29, 30.
} 
Lemma 4.7 (Order of growth of distinguished parameter). Let $X(z)=f(x) \frac{\partial}{\partial z}$ be an entire vector field on $\mathbb{C}$ without zeros, having finite class $\mathrm{p} \in \mathbb{N}$, and let $\omega_{X}, \Psi_{X}$ be the associated differential form and distinguished parameter, respectively. Then for $\infty \in \widehat{\mathbb{C}}$; the class of $X, \Psi_{X}$ and $\omega_{X}$ agree. Moreover, when either $X, \Psi_{X}$ or $\omega_{X}$ is of finite class $\mathrm{p}$, then

$$
\begin{aligned}
-(\mathrm{p}-\operatorname{order}(X))= & \mathrm{p}-\operatorname{order}\left(\Psi_{X}\right)=\mathrm{p}-\operatorname{order}\left(\omega_{X}\right), \\
& \mathrm{p}-\operatorname{type}\left(\omega_{X}\right)=\mathrm{p}-\operatorname{type}\left(\Psi_{X}\right) .
\end{aligned}
$$

Proof. Note that $X$ has an isolated essential singularity at infinity, $\omega_{X}$ is an entire differential form and $\Psi_{X}$ is a univalued entire function. $\Psi_{X}^{\prime}$ gives the expression of $\omega_{X}$ and $X=\left(1 / \Psi_{X}^{\prime}\right) \frac{\partial}{\partial z}$. The class, order and type of an entire function $\Psi_{X}$ and those of its derivative coincide; see [11], remark 4.5.2. Moreover, $1 / \Psi_{X}^{\prime}$ is without any zeros and of finite order, again following [11], remark 4.5.2; $f=1 / \Psi_{X}^{\prime}$ is of the same class and order as that of $\Psi_{X}$.

4.2. Residues and semi-residues. Let $((\mathbb{C}, 0), X)$ be a germ of singular analytic vector field having a zero or isolated essential singularity at $z=0$. The residue of $X$ is

$$
\operatorname{Res}(X, 0):=\operatorname{Res}\left(\omega_{X}, 0\right)=\frac{1}{2 \pi i} \int_{\gamma} \omega_{X},
$$

where $\gamma$ is a simple closed counterclockwise oriented path that encloses the origin.

Definition 4.8. Let $X$ be a singular analytic vector field on a Riemann surface $M$. The semi-residue associated with a pair of singularities $z_{j}, z_{k} \in \mathcal{S}_{X} \backslash \mathcal{Z}$ of $X$ is

$$
S\left(\omega, z_{j}, z_{k}, \gamma\right)=\int_{\gamma} \omega_{X}
$$

when it is finite, where $\gamma$ is a simple path that starts at $z_{j}$, ends at $z_{k}$ (i.e. $\gamma$ does not enclose; zeros, poles, essential singularities or accumulation points of them).

Note that we mainly use the case $z_{j} \neq z_{k}$; the pair of singularities $z_{j}, z_{k}$ could be two poles (related to saddle connections, homoclinic, heteroclinic trajectories; see [13], 23]), a pole and an isolated essential singularity or even an isolated essential singularity of 1-order greater than 1 , see for instance (8.9).

The semi-residue $S\left(\omega, z_{j}, z_{k}, \gamma\right)$, can be interpreted as the relative position, $\Psi_{X}\left(z_{k}\right)-\Psi_{X}\left(z_{j}\right) \in \mathbb{C}_{t}$, in the metric $g_{X}$ between the ramification values $\Psi_{X}\left(z_{j}\right)$ and $\Psi_{X}\left(z_{k}\right)$.

Moreover, they are of interest in the local scenario when the germ $((\mathbb{C}, 0), X)$ has an accumulation point of poles. In this case, the collection of semi-residues will be an infinite set, and a local invariant will be the tail end of the set. The semi-residues will also play a central role when studying the vector fields of the form $\mathrm{e}^{P(z)} \frac{\partial}{\partial z}$; see 8.1 . Figure [19, Theorem 8.16 and Theorem 8.31.

Example 4.9. Semi-residues and saddle connections. Let $X$ be a singular complex vector field on $M$, such that the flat metric $\left(M^{0}, g_{X}\right)$ contains an open maximal flat cylinder $S_{\rho}^{1} \times(0, h)$, of perimeter $\rho$ and finite height $h>0$ (also called finite height annulus flow 4 ). Two families of vector fields $X$ with this property are as follows.

\footnotetext{
${ }^{4}$ This notation follows the classical concepts of L. Markus; see 47] and Table 3in 411 Appendix for more details.
} 
i) If $X$ has only poles on a compact Riemann surface $M_{\mathfrak{g}}$ of genus $\mathfrak{g} \geq 2$, then $g_{X}$ has cylinders of this type; see [66] $\S 21$ pp. 107 and [48] lemma 1.6.

ii) Certain rational vector fields $X$ on $\widehat{\mathbb{C}}$ have cylinders of this type; see [50].

For these two families, on each boundary component of the closed cylinder $S_{\rho}^{1} \times$ $[0, h] \subset M$ there is at least one pole of $X$; say $z_{j} \in S_{\rho}^{1} \times\{0\}, z_{k} \in S_{\rho}^{1} \times\{h\}$. Moreover, there are an infinite number of geodesics $\gamma_{n}$ in $S_{\rho}^{1} \times[0, h]$, having extreme points $z_{j}, z_{k}$, nonhomotopically equivalent as paths between $z_{j}$ and $z_{k}$ (see [50] proof of theorem 3.2). Whence, $\left(M^{0}, g_{X}\right)$ has an infinite number of semi-residues,

$$
S\left(\omega_{X}, z_{j}, z_{k}, \gamma_{n}\right)=\int_{\gamma_{n}} \omega_{X}, \quad n \in \mathbb{Z}
$$

The geodesics $\left\{\gamma_{n}\right\}$ correspond to saddle connections of suitable rotated vector fields $\left\{\mathrm{e}^{i \theta_{n}} \mathfrak{R e}(X)\right\}$; see [50].

4.3. Asymptotic values. For meromorphic functions in the classical setting, asymptotic values appear in many instances (see [35], pp. 66, 53], pp. 298-303). We follow W. Bergweiler et al. [12, essentially verbatim from Definition 4.10 to Example 4.15, below.

Let $\Psi: \mathbb{C}_{z} \rightarrow \widehat{\mathbb{C}}_{t}$ be a meromorphic function, a priori not related to some vector field. The inverse function $\Psi^{-1}$ can be defined on a Riemann surface which is conformally equivalent to $\mathbb{C}$ via $\Psi^{-1}$. We want to study the singularities of $\Psi^{-1}$. This can be done by adding to $\mathbb{C}_{z}$ some ideal points and defining neighborhoods of these points.

Definition 4.10. Take $a \in \widehat{\mathbb{C}}_{t}$ and denote by $D(a, r)$ the disk of radius $r>0$ (in the spherical metric) centred at $a$. For every $r>0$ choose a component $U(r)$ of the preimage $\Psi^{-1}(D(a, r))$ in such a way that $r_{1}<r_{2}$ implies $U\left(r_{1}\right) \subset U\left(r_{2}\right)$. Note that the function $U: r \rightarrow U(r)$ is completely determined by its germ at 0 . Two possibilities can occur:

1. $\bigcap_{r>0} U(r)=\left\{z_{0}\right\}, z_{0} \in \mathbb{C}_{z}$. Then $a=\Psi\left(z_{0}\right)$. If $a \in \mathbb{C}_{t}$ and $\Psi^{\prime}\left(z_{0}\right) \neq 0$ or if $a=\infty$ and $z_{0}$ is a simple pole of $\Psi$, then $z_{0}$ is called an ordinary point. If $a \in \mathbb{C}_{t}$ and $\Psi^{\prime}\left(z_{0}\right)=0$ or if $a=\infty$ and $z_{0}$ is a multiple pole of $\Psi$, then $z_{0}$ is called a critical point and $a$ is called a critical value. We also say that the critical point $z_{0}$ lies over $a$.

2. $\bigcap_{r>0} U(r)=\varnothing$. Then we say that our choice $r \rightarrow U(r)$ defines a transcendental singularity of $\Psi^{-1}$. We also say that the transcendental singularity $U$ lies over $a$. For every $r>0$ the open set $U(r) \subset \mathbb{C}_{z}$ is called a neighborhood of the transcendental singularity $U$. So if $z_{k} \in \mathbb{C}_{z}$, we say that $z_{k} \rightarrow U$ if for every $\varepsilon>0$ there exists $k_{0}$ such that $z_{k} \in U(\varepsilon)$ where $k \geq k_{0}$.

Definition 4.11. If $U$ is a transcendental singularity, then $a$ is an asymptotic value, which means that there exists a path $\alpha \subset \mathbb{C}$ tending to $\infty$ such that $\Psi(z) \rightarrow a$ as $z \rightarrow \infty, z \in \alpha$. Such $\alpha$ is called an asymptotic path of $a_{j}$.

In particular, it follows that every neighborhood $U(r)$ of a transcendental singularity $U$ is unbounded. If $a$ is an asymptotic value of $\Psi$, then there is at least one transcendental singularity over $a$.

Certainly there can be many different transcendental singularities as well as critical and ordinary points over the same point $a$. We remark that if $\Psi$ is a meromorphic function and $D \subset \widehat{\mathbb{C}}_{t}$ contains no critical or asymptotic values then 
$\Psi: \Psi^{-1}(D) \rightarrow D$ is a covering. This justifies the name "transcendental singularities of $\Psi^{-1} \%$.

The following classification of asymptotic values is due to F. Iversen [38, although the connection between asymptotic values of $\Psi$ and transcendental singularities of $\Psi^{-1}$ was stated by A. Hurwitz [36].

Definition 4.12. A transcendental singularity $U$ over a is called direct if there exists $r>0$ such that $\Psi(z) \neq a$ for $z \in U(r)$ (then this is also true for all smaller values of $r$ ). A transcendental singularity $U$ over a is called indirect if it is not direct, i.e. for every $r>0$ the function $\Psi$ takes the value $a$ in $U(r)$.

In the case of an indirect transcendental singularity, the function $\Psi$ takes the value $a$ infinitely often in $U(r)$.

Definition 4.13. We say that $U$ is a logarithmic branch point over a if $\Psi: U(r) \rightarrow$ $D(a, r) \backslash\{a\}$ is a universal covering for some $r>0$. The (unbounded) neighborhoods $U(r)$ are called exponential tracts.

Example 4.14. The simplest case of a direct transcendental singularity is a logarithmic branch point. If $\Psi(z)=\exp (z)$, then its inverse, the (multivalued) function $\Psi^{-1}(t)=\log (t)$ has two logarithmic branch points: one over 0 and another over $\infty$. Of course 0 and $\infty$ are asymptotic values of $\Psi$ and, for $R>0, U_{0}(R)=$ $\{z \mid \mathfrak{R e}(z)<R\}$ and $U_{\infty}(R)=\{z \mid \mathfrak{R e}(z)>R\}$, are exponential tracts for the asymptotic values 0 and $\infty$, respectively.

Example 4.15. A simple example of an indirect transcendental singularity is given by the inverse function of $\Psi(z)=\sin (z) / z$. Note that, the asymptotic value 0 is a limit point of critical values.

In $\$ 5 \sqrt{6}$ and $₫ 7$ we will be interested in applying some of the above definitions to germs $\left((V \backslash\{q\}, q), \Psi_{X}\right)$ of the distinguished parameter $\Psi_{X}$, associated to a singular analytic vector field $X$ on an arbitrary Riemann surface $M$ as in diagram (2.6), where $q$ will be an isolated singularity of $\Psi_{X}$.

As a first observation, note that if $q$ is an isolated essential singularity of $X$, then $q$ is an isolated essential singularity of $\Psi_{X}$ and there is at least one finite asymptotic value of $\Psi_{X}$ associated to $q$.

Example 4.16. Consider $X(z)=\mathrm{e}^{z} \frac{\partial}{\partial z}$, hence $\Psi_{X}(z)=\int_{0}^{z} \omega_{X}=1-\mathrm{e}^{-z}$ has asymptotic values $1, \infty \in \widehat{\mathbb{C}}_{t}$ ( see $\$ 5.3 .1$ for full details). Of course $q=\infty \in \widehat{\mathbb{C}}_{z}$ is an isolated essential singularity of $X$ and also of $\Psi_{X}$. The (multivalued) function $\Psi_{X}^{-1}(t)=-\log (1-t)$ has two logarithmic branch points: one over 1 and the other over $\infty$.

Our second observation is that poles of $X$ correspond to critical points of $\Psi_{X}$.

The case of zeros of $X$ is more involved: When $q$ is a zero of $X$, we need to consider the associated differential form $\omega_{X}$.

i) If $\operatorname{Res}\left(\omega_{X}, q\right)=0$, then $\Psi_{X}$ is univalued and $q$ is a pole of $\Psi_{X}$.

ii) If $\operatorname{Res}\left(\omega_{X}, q\right) \neq 0$, then $\Psi_{X}$ is multivalued and additively automorphic. In this case either $q$ is a simple zero of $X$ so $\Psi_{X}$ has a logarithmic branch point over $q$ (the logarithmic branch point is of $\Psi_{X}$, not of $\Psi_{X}^{-1}$ ), or $q$ is a multiple zero of $X$ in which case $q$ is a pole of $\Psi_{X}$.

The reverse implications are straightforward using Laurent series.

The above is summarized in Table 1 . 
TABLE 1. Relationship between $X$ and $\Psi_{X}$ in terms of their singularities and values.

\begin{tabular}{|c|c|}
\hline $\begin{array}{c}\text { Behaviour of } X \\
\text { in a neighborhood of } q\end{array}$ & $\begin{array}{c}\text { Behaviour of } \Psi_{X} \\
\text { in a neighborhood of } q\end{array}$ \\
\hline$q$ is a pole of $X$ & $\bar{q} q$ is a critical point of $\Psi_{X}$ \\
\hline $\begin{array}{l}q \text { is a simple zero of } X \\
\quad\left(\text { so } \operatorname{Res}\left(\omega_{X}, q\right) \neq 0\right)\end{array}$ & $\begin{array}{c}\Psi_{X} \text { has a logarithmic branch point over } q \\
\text { and } \Psi_{X} \text { is multivalued and additively automorphic }\end{array}$ \\
\hline $\begin{array}{c}q \text { is a multiple zero of } X \\
\operatorname{Res}\left(\omega_{X}, q\right)=0 \\
\operatorname{Res}\left(\omega_{X}, q\right) \neq 0\end{array}$ & $\begin{array}{c}q \text { pole of } \Psi_{X}: \\
\Psi_{X} \text { is univalued, } \\
\Psi_{X} \text { is multivalued and additively automorphic }\end{array}$ \\
\hline $\begin{array}{c}q \text { is an isolated } \\
\text { essential singularity } \\
\text { of } X\end{array}$ & $\begin{array}{c}q \text { is an isolated essential singularity of } \Psi_{X} \\
\text { and there exists at least one finite asymptotic } \\
\text { value } a \in \mathbb{C} \text { of } \Psi_{X} \text { associated to } q\end{array}$ \\
\hline
\end{tabular}

Thus, the singular set5 $5 \mathcal{S}\left(\Psi_{X}\right) \neq \mathcal{S}_{X}$, since $\mathcal{P}$, the set poles of $X$, does not in general correspond to a singularity of $\Psi_{X}$, and the set of zeros of $\Psi_{X}$ does not in general correspond to a singularity of $X$. However, $\overline{\mathcal{Z} \cup E} \subset \mathcal{S}\left(\Psi_{X}\right)$.

Example 4.17. Consider $X(z)=\frac{\mathrm{e}^{z^{3}}}{3 z^{3}-1} \frac{\partial}{\partial z}$ on $\widehat{\mathbb{C}}_{z}$, note that $X$ has poles at $\left\{\frac{1}{\sqrt[3]{3}}, \frac{\mathrm{e}^{i 2 \pi / 3}}{\sqrt[3]{3}}, \frac{\mathrm{e}^{-i 2 \pi / 3}}{\sqrt[3]{3}}\right\}$. On the other hand a simple calculation shows that

$$
\Psi_{X}(z)=\int_{0}^{z} \omega_{X}=-z \mathrm{e}^{-z^{3}}
$$

so the poles of $X$ are critical points of $\Psi_{X}$ with critical values $\left\{-\frac{1}{\sqrt[3]{3 \mathrm{e}}},-\frac{\mathrm{e}^{i 2 \pi / 3}}{\sqrt[3]{3 \mathrm{e}}}\right.$, $\left.-\frac{\mathrm{e}^{-i 2 \pi / 3}}{\sqrt[3]{3 \mathrm{e}}}\right\}$. Thus, $\mathcal{S}\left(\Psi_{X}\right) \neq \mathcal{S}_{X}$. Also, $X$ has an isolated essential singularity at $\infty \in \widehat{\mathbb{C}}_{z}$ and $a_{1}=0$ is its (finite) asymptotic value with multiplicity 3 , with the exponential tracts given by

$$
\begin{aligned}
& A_{1}=\{z \in \mathbb{C} \mid \arg (z) \in[-\pi / 6, \pi / 6]\}, \\
& A_{2}=\{z \in \mathbb{C} \mid \arg (z) \in[\pi / 2,5 \pi / 6]\}, \\
& A_{3}=\{z \in \mathbb{C} \mid \arg (z) \in[7 \pi / 6,3 \pi / 2]\} .
\end{aligned}
$$

See Figure 21, b for the phase portrait.

As a summary of the material presented in this section we have.

Corollary 4.18 (Local and global invariants). Let $X$ be an analytic vector field on a Riemann surface $M$, having an isolated singularity at $q$, numerable critical and asymptotic values of $\Psi_{X}$. The following are analytic invariants of $X$ :

1) Finite or infinite class, order of growth, order of vanishing for $((M, q), X)$.

2) Residues for $((M, q), X)$.

3) Semi-residues for $(M, X)$.

4) Configuration of critical and asymptotic values of $\Psi_{X}$ modulo translations, for $(M, X)$.

As a prelude of the argument in part (4), we introduce an illustrative family.

\footnotetext{
${ }^{5}$ Recall Definitions 2.1 and 2.4
} 
Example 4.19. Pullback vector fields on hyperelliptic Riemann surfaces. Let $M$ be a hyperelliptic compact Riemann surface of genus $\mathfrak{g} \geq 1$, i.e. $M$ is provided with a meromorphic function $\Psi: M \rightarrow \widehat{\mathbb{C}}_{t}$ of degree 2 ; see [51. Let $\Psi\left(q_{j}\right)=$ $\left\{a_{j}\right\}=\left\{a_{1}, \ldots, a_{2 \mathfrak{g}+2}\right\} \subset \widehat{\mathbb{C}}_{t}$ be the critical values of $\Psi$. It is well known that the configuration of critical values up to $\operatorname{PSL}(2, \mathbb{C})$-action, determines the hyperelliptic $(M, \Psi)$; see [7, pp. 388, and [51], Lecture I.

For a moment we assume that $\infty \neq a_{j}$, and furnish the target with $\left(\widehat{\mathbb{C}}, \frac{\partial}{\partial t}\right)$.

Let $X=\Psi^{*}\left(\frac{\partial}{\partial t}\right)$ be a meromorphic vector field on $M$, whose singularities $\mathcal{S}_{X}$ are $2 \mathfrak{g}+2$ poles of order -1 at $\left\{q_{j}\right\}$, and 2 zeros of order 2 at $\left\{p_{\iota}\right\}=\Psi^{-1}(\infty)$. Thus, $(M, \Psi)$ determines $(M, X)$ and Corollary 4.18,3 holds.

Moreover, the configuration of critical values $\left\{a_{j}\right\}$ is a complete analytic invariant of $(M, X)$.

Let $(M, X),(N, Y)$ be two pullback vector fields on hyperelliptic surfaces. Assume that there exists a biholomorphism $\Upsilon: M \rightarrow N$ with $\Upsilon_{*} X=Y$. We shall now prove that the critical values $\left\{a_{1}^{\prime}, \ldots, a_{2 \mathfrak{g}+2}^{\prime}\right\}$ of $\Psi_{Y}$ coincide with the critical values of $\Psi_{X}$ up to a global translation $T: \mathbb{C}_{t} \rightarrow \mathbb{C}_{t}$.

If $\Psi_{X, \mathrm{j}}: V_{\mathrm{j}} \rightarrow \mathbb{C}_{t}$ is a local biholomorphism with $\Psi_{X, \mathrm{j}}\left(q_{0}\right):=t_{0} \in \mathbb{C}_{t}$, then from the diagram

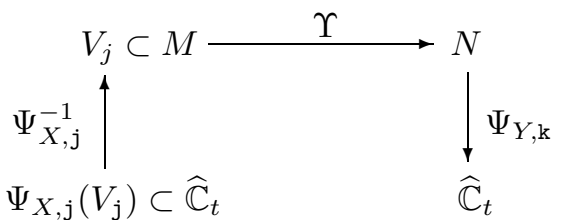

the map $T_{\mathrm{jk}}:=\Psi_{Y, \mathrm{k}} \circ \Upsilon \circ \Psi_{X, \mathrm{j}}^{-1}$ is the restriction of a translation $\{z \mapsto z+b\}$ in $\left(\widehat{\mathbb{C}}, \frac{\partial}{\partial t}\right)$. The analytic continuation $T$ of $T_{j \mathrm{k}}$ is well defined for every path $\gamma \subset$ $\widehat{\mathbb{C}}_{t} \backslash\left\{a_{j}\right\}$ (without loss of generality, we assume that there are not three points $t_{0}, a_{j}, a_{\ell}$ in a straight line of $\left.\mathbb{C}_{t}\right)$. Consider the semi-residues

$$
S\left(\omega_{X}, q_{0}, q_{j}, \gamma_{j}\right)=\int_{\gamma_{j}} \omega_{X}=a_{j}-t_{0},
$$

where $\gamma_{j}$ is any of the two components in the inverse image $\Psi^{-1}\left(\overline{t_{0} a_{j}}\right) \subset M$, of the oriented straight line segment $\overline{t_{0} a_{j}}$.

The analytic continuation of $T$ along the union of paths $\bigcup_{j} \overline{t_{0} a_{j}} \subset \widehat{\mathbb{C}}_{t}$, is the restriction of a translation.

Under $T$ the configuration of critical values of $\Psi_{Y}$ coincide with the critical values of $\Psi_{X}$, as in Corollary 4.18, 4 . The case $a_{j}=\infty$, is left as an exercise for the reader.

Proof of Corollary 4.18,4. Let $(M, X)$ be the vector field and its global $\Psi_{X}$. The configuration of critical and finite asymptotic values $\left\{a_{1}, a_{2}, \ldots\right\} \subset \widehat{\mathbb{C}}$ is a numerable set. There exist a regular value $t_{0}=\Psi_{X, j}\left(q_{0}\right)$ such that there are not three points $t_{0}, a_{j}, a_{\ell}$ in a straight line of $\mathbb{C}_{t}$. Then we consider the analytic continuation $T$ of the a respective $T_{\mathrm{jk}}$, analogous to (4.2), along the union of paths $\bigcup_{j} \overline{t_{0} a_{j}} \subset \mathbb{C}_{t}$. Using arguments as in the example, the conclusion follows.

The following examples show that the numerable hypothesis is needed, and that the set of critical values of $\Psi_{X}$ is not a complete analytic invariant of $X$. 
Example 4.20. W. Gross constructed examples of analytic functions having dense sets of critical and asymptotic values (see [29], also [53, pp. 287), hence the numerable assumption in Corollary 4.18 is necessary.

Obviously, for compact $M$ and (even in the case of) a meromorphic map $\Psi_{X}$, the set of critical values does not determine $M$ (and by consequence $X=\Psi_{X}^{*}\left(\frac{\partial}{\partial t}\right)$ ). Roughly speaking, G. V. Belyǐ [9] showed that nonsingular algebraic curves $M$ are defined by polynomials having algebraic number coefficients if and only if $M$ can be expressed as a ramified covering having only $\{0,1, \infty\} \subset \widehat{\mathbb{C}}$ as critical values.

\section{Angular SeCtors at AN isolated EsSential Singularity}

5.1. Angular sectors of vector fields in the real analytic category. Very roughly speaking, let $\left(\left(\mathbb{R}^{2}, \overline{0}\right), Z\right)$ be a real analytic vector field germ having an isolated zero at the origin, and let $\mathcal{F}$ be its associated singular planar real analytic foliation. Sometimes the local behaviour of $\mathcal{F}$ can be described by using hyperbolic, elliptic, parabolic (real analytic) angular sectors, prototyped from the vector fields

1) $Z_{h}=x \frac{\partial}{\partial x}-y \frac{\partial}{\partial y}$

2) $Z_{e}=\left(x^{3}-3 x y^{2}\right) \frac{\partial}{\partial x}+\left(3 x^{2} y-y^{3}\right) \frac{\partial}{\partial y}$

3) $Z_{p}=x \frac{\partial}{\partial x}+y \frac{\partial}{\partial y}$ on $\{(x, y) \mid 0 \leq x, y<1, x y<1 / 2\}$,

on $\left\{(x, y) \mid 0 \leq x, y<1, x^{2}+y^{2}<1\right\}$, on $\left\{(x, y) \mid 0 \leq x, y<1, x^{2}+y^{2}<1\right\}$,

respectively. The three left drawings in Figure 4 give a topological representation of these kinds of angular sectors. The following nice result is well known.

Theorem 5.1 (A. A. Andronov, E. A. Lentovich, I. I. Gordon, A. G. Mayer, F. Dumortier). A singular planar real analytic foliation $\mathcal{F}$ on $\left(\mathbb{R}^{2}, \overline{0}\right)$ with a characteristic trajectory admits a sectorial decomposition into finitely many sectors, hyperbolic, elliptic and/or parabolic, separated by characteristic trajectories.

Roughly speaking, a characteristic trajectory of $\mathcal{F}$ accumulates to the origin with a well-defined slope. See [6], ch. VIII, [8], pp. 86 and [37], pp. 149 for precise statements and sketch of the proof.

5.2. Angular sectors $C, H, E, P, \mathscr{E}$ of vector fields in the complex analytic category. We want to consider a singular analytic vector field germ $((\mathbb{C}, 0), X)$, having an isolated singularity at 0 . Let us introduce the following conventions.

Consider the closed upper half-plane $\overline{\mathbb{H}}_{+}^{2}=\{z \mid \mathfrak{I m}(z) \geq 0\} \cup\{\infty\} \subset \widehat{\mathbb{C}}$, or the lower half-plane $\overline{\mathbb{H}}_{-}^{2}$ for $\mathfrak{I m}(z) \leq 0$; any of them is called $\overline{\mathbb{H}}^{2}$. Moreover, $\overline{\mathbb{H}}^{2}$ is provided with the singular flat metric inherited from $\left(\widehat{\mathbb{C}}, \frac{\partial}{\partial z}\right)$. For $q=0$ or $\infty$ in the boundary $\mathbb{R} \cup\{\infty\}$ of $\overline{\mathbb{H}}^{2}$, the pair $\left(\overline{\mathbb{H}}^{2}, q\right)$ denotes a domain for germs.

Let $\nu=\nu_{1}+i \nu_{2} \in \mathbb{C} \backslash \mathbb{R}$ be a number and $\overline{0 \nu} \subset \mathbb{C}$ the corresponding straight line segment. When $\nu_{2}<0, \nu$ determines a horizontal right strip

$$
S_{\nu}=\left\{z \in \mathbb{C} \mid \nu_{2} \leq \mathfrak{I m}(z) \leq 0, \mathfrak{R e}(z)>\text { the respective } x \in \overline{0 \nu}\right\} \cup\{\infty\}
$$

or horizontal left strip, when $\nu_{2}>0$,

$$
S_{\nu}=\left\{z \in \mathbb{C} \mid 0 \leq \mathfrak{I m}(z) \leq \nu_{2}, \mathfrak{R e}(z)<\text { the respective } x \in \overline{0 \nu}\right\} \cup\{\infty\} .
$$

We provide each strip $\left(S_{\nu}, \infty\right)$ with the singular flat metric inherited from $\left(\widehat{\mathbb{C}}, \frac{\partial}{\partial z}\right)$. Note that $S_{\nu}$ is a pair, a Riemannian manifold with boundary and a displacement number $\nu$. 
Definition 5.2. Let $((\mathcal{A}, q), X)$ be a representative of a germ of angular sector modelled on:

1. A center

2. A hyperbolic sector

3. An elliptic sector

4. A parabolic sector

5. A class 1 entire sector

Here, $\overline{\mathbb{H}}^{2}$ denotes an upper or lower half-plane.

We will omit the sub-index in $C_{2 \pi / \lambda_{2}}, P_{\nu}$, when the context is clear. The geometric meaning of $2 \pi / \lambda_{2}$ and $\nu$ will be explained in (5.6).

5.3. Attributes of the angular sectors. By simple inspection we note that germ representatives in Definition 5.2 enjoy the following features.

1) $(\mathcal{A}, q) \subset \widehat{\mathbb{C}}_{z}$ is a Riemann surface with boundary

$$
\partial \mathcal{A}=\{q\} \cup \sigma_{1} \cup \gamma \cup \sigma_{2} .
$$

Here, $\gamma$ will be a piecewise $C^{1}$ path (which will have to coincide with a trajectory of $\mathfrak{R e}(X)$ only in the center case $\left.C_{2 \pi / \lambda_{2}}\right)$.

Moreover, $q=0$ in the center $C_{2 \pi / \lambda_{2}}$ or hyperbolic $H$ cases, $q=\infty$ otherwise.

2) The path $\{q\} \cup \sigma_{1} \cup \gamma \cup \sigma_{2}$ is oriented in the counterclockwise sense $(\mathcal{A}$ has a canonical orientation).

3) The distinguished parameter $\Psi:((\mathcal{A}, q), X) \rightarrow\left(\widehat{\mathbb{C}}, \frac{\partial}{\partial t}\right)$ can be chosen as below: for $C_{2 \pi / \lambda_{2}}$ as a (multivalued) logarithm;

for $H, E, P_{\nu}$ as the identity $\Psi(z)=z$;

for $\mathscr{E}$ as $\Psi(z)=\int_{0}^{z} \mathrm{e}^{-\zeta} d \zeta$, we will explore this in $\$ 5.3 .1$

4) In cases different from a center $C_{2 \pi / \lambda_{2}}$,

$$
\sigma_{\iota}(\tau): \mathcal{I}_{\iota} \longrightarrow \mathcal{A} \backslash\{q\}, \quad \iota=1,2,
$$

are trajectories of $\mathfrak{R e}(X)$, where $\mathcal{I}_{\iota}=\left(\tau_{\min }, \tau_{\max }\right) \subset \mathbb{R}$ is the maximal interval of definition. The $\alpha$ or $\omega$-limit of $\sigma_{\iota}(\tau)$ is $q$.

$\sigma_{1}, \sigma_{2}$ will play the role of characteristic trajectories 6 (analogous to Theorem 5.1).

5) The maximal intervals of definition $\mathcal{I}_{1}, \mathcal{I}_{2}$ satisfy the following:

for $H$, both $\mathcal{I}_{1}, \mathcal{I}_{2}$ are bounded (i.e. finite) intervals in $\mathbb{R}$;

for $E, P_{\nu}$, both $\mathcal{I}_{1}, \mathcal{I}_{2}$ are unbounded intervals;

for $\mathscr{E}$, exactly one $\mathcal{I}_{\iota}$ is a bounded interval, equation (5.2) will show this.

The class 1 entire sector $\mathscr{E}$ is more elaborate.

\footnotetext{
6 This concept of characteristic trajectory is weaker than that of A. A. Andronov et al. since our characteristic trajectory can be a spiral trajectory at the singularity. Since we are dealing only with isolated essential singularities $((\mathbb{C}, 0), X)$, in $\widehat{\mathbb{C}}_{t}$ there are always horizontal trajectories that arrive to $\infty \in \widehat{\mathbb{C}}_{t}$, by pulling back via $\Psi$ we obtain a characteristic trajectory $\sigma$ for $((\mathbb{C}, 0), X)$.
} 
5.3.1. Class 1 entire sectors $\mathscr{E}=\left(\left(\overline{\mathbb{H}}^{2}, \infty\right), e^{z} \frac{\partial}{\partial z}\right)$. We make two different choices for the representative $(\mathcal{A}, \infty),\left(\mathcal{A}^{\prime}, \infty\right) \subset\left(\overline{\mathbb{H}}^{2}, \infty\right)$ of this germ, the second will be invariant under $\mathfrak{R e}\left(\mathrm{e}^{z} \frac{\partial}{\partial z}\right)$. Both representatives will appear in different situations along the work.

First representative: Let $\mathcal{A} \subset \overline{\mathbb{H}}_{+}^{2}$ be a sector in the upper half-plane, as in the left-hand side of Figure 3, (for the lower half-plane, details change in an obvious way). The distinguished parameter is

$$
\Psi(z)=1-\mathrm{e}^{-z}=\int_{0}^{z} \mathrm{e}^{-\zeta} d \zeta: \mathcal{A} \rightarrow \widehat{\mathbb{C}}_{t} .
$$

As characteristic trajectories, we use $\left.\sigma_{1}(\tau)=-\log (-\tau):(\infty,-3)\right] \rightarrow \mathcal{A}$, with $(\infty,-\log (3)]$ being its image, and $\sigma_{2}(\tau)=-\log (-\tau):[-1,0) \rightarrow \mathcal{A}$, with image $[0, \infty)$.

Both images under $\Psi$ are inside of $\mathbb{R} \cup\{\infty\} \subset \widehat{\mathbb{C}}_{t}$. The respective times it takes for a characteristic trajectory to reach the singularity $\infty \in \mathcal{A}$ of the vector field are

$$
\int_{-\infty}^{-\log (3)} \mathrm{e}^{-\zeta} d \zeta=\infty, \quad \text { and } \quad \int_{0}^{+\infty} \mathrm{e}^{-\zeta} d \zeta=1 .
$$

Let $\gamma=\gamma_{1} \cup \gamma_{2}$ be in the boundary of $\mathcal{A}$, here; $\gamma_{1}$ is a path that starts at $-\log (3)$ and ends at $i \pi$ and $\gamma_{2}$ is the vertical segment from $i \pi$ to 0 ; they determine the germ representative $\mathcal{A} \subset \overline{\mathbb{H}}_{+}^{2}$; see left drawings in Figure 3. It is to be noted that this choice of $\gamma$ in the boundary of $\mathcal{A}$ is highly nongeneric in the sense that it is touching one of the horizontal homoclinic trajectories, $\{\mathfrak{I m}(z)=i k \pi, k \in \mathbb{N}\}$ when viewed from 0 , with endpoints at $\infty \in \mathcal{A}$. Homoclinic means it is a trajectory which joins $\infty$ to itself. However, as will be shown in Remark [5.18,2, $\gamma$ can be deformed inside $\mathcal{A}$ so as to obtain a path as the one just described.

The image of the boundary of $\mathcal{A}, \Psi\left(\sigma_{1} \cup \gamma_{1} \cup \gamma_{2} \cup \sigma_{2}\right) \subset \widehat{\mathbb{C}}_{t}$ with the orientation inherited from $\sigma_{1} \cup \gamma_{1} \cup \gamma_{2} \cup \sigma_{2}$, is as follows:

$$
\Psi\left(\sigma_{1}\right)=(\infty,-2]
$$

$\Gamma_{1}=\Psi\left(\gamma_{1}\right)$ is an arc of a circle centred at 0 starting at -2 and ending at 2 ;

$\Gamma_{2}=\Psi\left(\gamma_{2}\right)$ is an arc of a circle centred at 1 starting at 2 and ending at 0 ; $\Psi\left(\sigma_{2}\right)=[0,1)$;

see right drawing in Figure 3 .

Definition 5.3. 1. Let $\Gamma=\Gamma_{1} \cup \Gamma_{2}$ be any simple piecewise $C^{1}$ path in $\left(\mathbb{C}, \frac{\partial}{\partial t}\right)$ parametrized by $\Gamma_{1}:\left[\tau_{1}, \tau_{2}\right] \rightarrow \mathbb{C}_{t}, \Gamma_{2}:\left[\tau_{2}, \tau_{3}\right] \rightarrow \mathbb{C}_{t}$, where $\Gamma_{1}\left(\tau_{2}\right)=\Gamma_{2}\left(\tau_{2}\right)=$ $x_{0}+i y_{0}$.

$\Gamma$ bounces off (the horizontal trajectory $\left\{t \mid \mathfrak{I m}(t)=y_{0}\right\}$ ) if:

a) the endpoints $t_{j}=\Gamma\left(\tau_{j}\right), j=1,2,3$, have the same imaginary part $y_{0}$ and either $\mathfrak{R e}\left(t_{1}\right), \mathfrak{R e}\left(t_{3}\right)<\mathfrak{R e}\left(t_{2}\right)$ or $\mathfrak{R e}\left(t_{1}\right), \mathfrak{R e}\left(t_{3}\right)>\mathfrak{R e}\left(t_{2}\right)$;

b) $\Gamma$ is contained in the same upper/lower half-plane $\left\{t \mid \mathfrak{I m}(t) \geq y_{0}\right\}$ (resp. $\leq)$.

2. Analogously, given a local parameter $\Psi_{X}$ as in $\$ 2$, a path $\gamma$, in the domain of $\Psi_{X}$, bounces off iff its image $\Psi_{X}(\gamma)$ in $\left(\mathbb{C}, \frac{\partial}{\partial t}\right)$ bounces off.

The concept of "bounce off" will enable us to recognize the presence of entire sectors in 9 . 
Note that the path $\gamma_{1} \cup \gamma_{2}$ bounces off the horizontal homoclinic trajectory that contains $i \pi$ and its image $\Gamma_{1} \cup \Gamma_{2}$ bounces off the horizontal trajectory (in $\mathbb{C}_{t}$ ) that contains 2 .

The discontinuity in the image of $\Psi\left(\sigma_{2}\right) \cup \Psi\left(\sigma_{1}\right)$, i.e. the jump from 1 to $-\infty$, is due to the fact that $\Psi$ has two asymptotic values:

$$
\lim _{\tau \rightarrow-\infty} \Psi\left(\sigma_{1}(\tau)\right)=\infty \in \widehat{\mathbb{C}}_{t}, \quad \lim _{\tau \rightarrow-\infty} \Psi\left(\sigma_{2}(\tau)\right)=1 \in \widehat{\mathbb{C}}_{t}, \quad \tau \in \mathbb{R}^{-} .
$$

The second asymptotic value 1 is not intrinsic, however the fact that it is finite is intrinsic. The reader can verify the coherence of Figures 1 and 3 ,

Second representative: We make the choice $\mathcal{A}^{\prime}=\{z \mid k \pi \leq \mathfrak{I m}(z)\}$, see the light grey disk in the bottom left drawing, Figure $3, \mathcal{A}^{\prime}$ is invariant under $\mathfrak{R e}\left(\mathrm{e}^{z} \frac{\partial}{\partial z}\right)$, whence $\gamma_{1}, \gamma_{2}$ have reduced to a point.

A remarkable property now becomes apparent: $\Psi: \mathcal{A}^{\prime} \rightarrow \widehat{\mathbb{C}}_{t}$ in (5.1), determines a "half-logarithmic spiral", whose description requires two preliminary concepts in the general framework.

Definition 5.4. A semi-infinite helicoid is a Riemann surface with boundary obtained from a semi-infinite succession of half-planes 7

$$
\overline{\mathbb{H}}_{ \pm}^{2} \cup \overline{\mathbb{H}}_{ \pm}^{2} \cup \ldots
$$

glued together along their boundaries as is usual in the graph of $z \mapsto \exp (z)$. Analogously, a finite helicoid is obtained with an even finite succession of half-planes

$$
\overline{\mathbb{H}}_{ \pm}^{2} \cup \ldots \cup \overline{\mathbb{H}}_{\mp}^{2}
$$

glued similarly, here $\overline{\mathbb{H}}_{ \pm}^{2}$ means $\left(\overline{\mathbb{H}}_{ \pm}^{2}, \frac{\partial}{\partial t}\right)$.

For the readers convenience, the glueing alluded in the definition will be made clear in Corollary 5.11 .

Referring to the top left and right drawings in Figure 3, for each horizontal strip

$$
B_{k}=\left((\{z \mid k \pi \leq \mathfrak{I m}(z) \leq(k+1) \pi\}, \infty), \mathrm{e}^{z} \frac{\partial}{\partial z}\right) \subset \mathcal{A}^{\prime}, \quad k \geq 1,
$$

its image $\Psi\left(B_{k}\right)$ is a lower half-plane $\overline{\mathbb{H}}_{-}^{2}$ when $k$ is odd or an upper half-plane $\overline{\mathbb{H}}_{+}^{2}$ when $k$ is even.

Definition 5.5. Let $\Psi$ be as in equation (5.1), a half-logarithmic spiral associated to the asymptotic values $a=1, \infty$ is the Riemann surface of

$$
\Psi: \bigcup_{k \geq 1} B_{k} \longrightarrow\left(\mathbb{C}^{*}, \frac{\partial}{\partial t}\right)
$$

Remark 5.6. Note that a semi-infinite helicoid is the same Riemann surface as a half-logarithmic spiral, however, they arise from apparently different contexts; the semi-infinite helicoid arises from the isometric gluing of a succession of halfplanes $\left(\overline{\mathbb{H}}_{ \pm}^{2}, \frac{\partial}{\partial t}\right)$, and the half-logarithmic spiral arises as the Riemann surface of $\left(\overline{\mathbb{H}}_{ \pm}^{2}, \mathrm{e}^{z} \frac{\partial}{\partial z}\right)$; see Table 3 in $₫ 11$ Appendix.

\footnotetext{
${ }^{7}$ This notation follows the classical concepts of L. Markus for phase portraits of real vector fields; see [4] and Table 3 in $\$ 11$ Appendix for more details.
} 


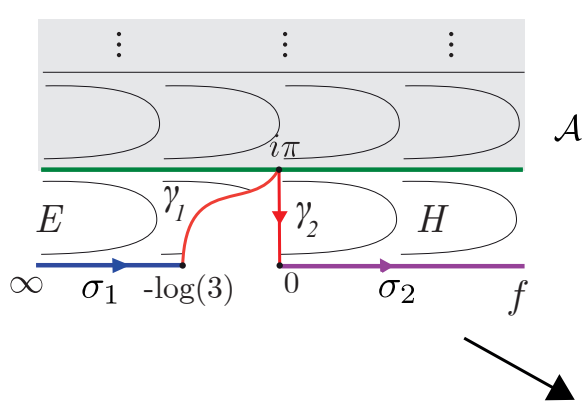

$$
\Psi(z)=\int_{0}^{z} e^{-\zeta} d \zeta
$$

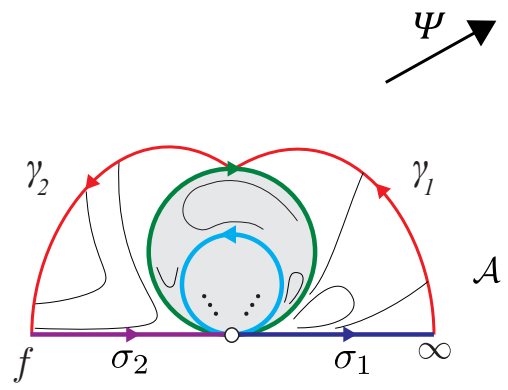

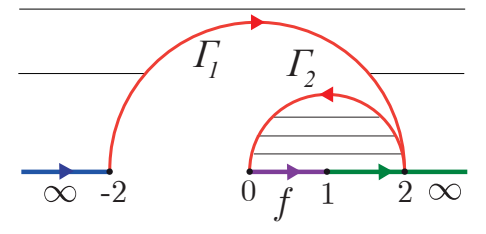
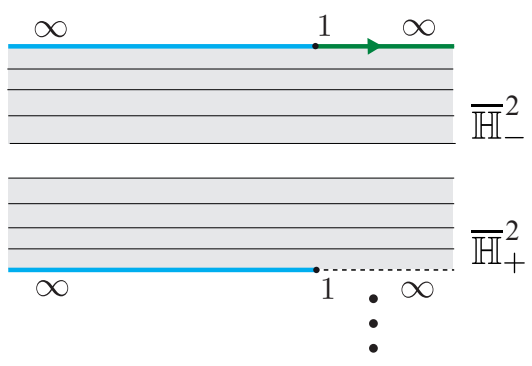

Figure 3. Class 1 entire sector. The sector $\mathscr{E}=\left(\left(\overline{\mathbb{H}}^{2}, \infty\right), \mathrm{e}^{z} \frac{\partial}{\partial z}\right)$, is illustrated viewed from 0 (left upper drawing) and from $\infty$ (left bottom drawing) in $\overline{\mathbb{H}}_{+}^{2}$. The right drawing describes its image $\Psi(\mathcal{A})$. The path $\gamma=\gamma_{1} \cup \gamma_{2}$ is chosen so that its image $\Psi\left(\gamma_{1} \cup \gamma_{2}\right)=\Gamma_{1} \cup \Gamma_{2}$ determines two semi-circles, and it bounces off the horizontal trajectory $\{\mathfrak{I m}(t)=0\}$ that contains the finite asymptotic value $a=1$ and the asymptotic value $\infty$. In $\mathcal{A}$ : the dark blue line segment is an infinite length trajectory $\sigma_{1}$ that starts at $-\infty$ and ends at the beginning of the arc $\gamma_{1}$; while the purple line segment $\sigma_{2}$ is a finite length trajectory that starts at the end of $\gamma$ and ends at $\infty$. In $\Psi(\mathcal{A})$ : the images under $\Gamma_{1} \cup \Gamma_{2}$ conserves the same colors. The purple line segment $\Psi\left(\sigma_{2}\right)$ starts at 0 (the end of $\Gamma$ ) and ends at the finite asymptotic value $a=1$. Furthermore: the closed set $E \subset \mathcal{A}$ determines an elliptic sector; $H \subset \mathcal{A}$ determines a hyperbolic sector. The image of the strip $\{\pi \leq \mathfrak{I m}(z) \leq 2 \pi\} \subset \mathcal{A}$, determines the lower half-plane $\overline{\mathbb{H}}_{-}^{2}$, which in turn is glued (as will be made clear in Corollary 5.11) to another half-plane $\overline{\mathbb{H}}_{+}^{2}$ by the complementary half-ray (light blue). These pieces are repeated infinitely further forming a half-logarithmic spiral (shown as light grey) associated to the finite asymptotic value $a=1$ and the asymptotic value $\infty$. As a metric property; finite $g_{X}$-length trajectories in the boundaries are stressed with sub-index $f$, infinite $g_{X}$-length trajectories with sub-index $\infty$. See $\$ 5.3 .1$ 
Furthermore, if we consider $\mathscr{E}$ as in Definition 5.2 using $\mathcal{A}^{\prime}=\{z \mid k \pi \leq \mathfrak{I m}(z)\}$, then

$$
\mathscr{E}=\left(\left(\mathcal{A}^{\prime}, \infty\right), \mathrm{e}^{z} \frac{\partial}{\partial z}\right) \cong\{\text { semi-infinite helicoid }\} \cong\{\text { half-logarithmic spiral }\},
$$

where $\cong$ means biholomorphism as pairs: Riemann surfaces and vector fields.

$\mathcal{A}$

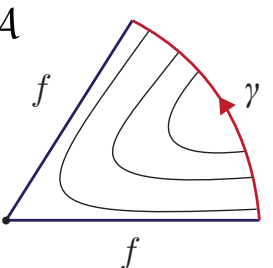

$\mathcal{A}$

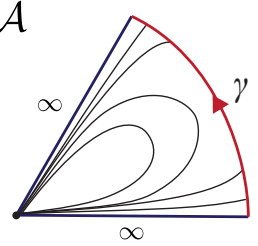

$\mathcal{A}$

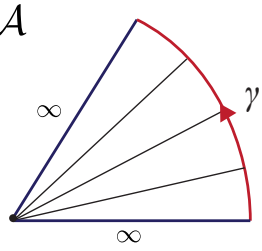

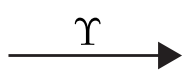

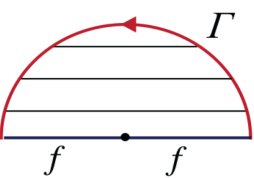

$H$
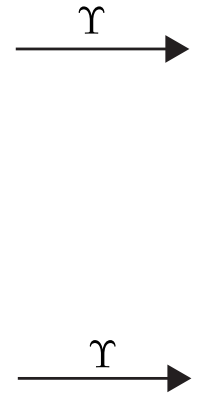

$E$

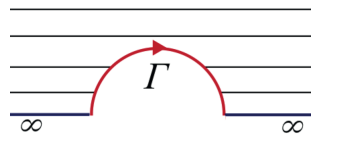

$\infty$

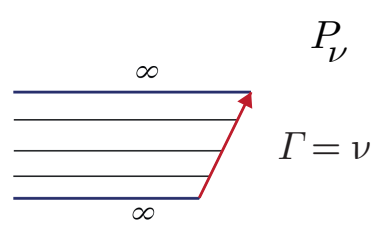

FIGURE 4. Hyperbolic, elliptic and parabolic sectors (in the complex analytic category) of a vector field $X$ on a Riemann surface $M$. There exists a biholomorphism $\Upsilon:((\mathcal{A}, q), X) \subset M \rightarrow W$, for $W \in\left\{H, E, P_{\nu}\right\}$, which are angular sectors as in Definition 5.2. As a metric property; finite $g_{X}$-length trajectories that start or end at the singularity $q$ are stressed with sub-index $f$, infinite $g_{X}$-length trajectories with sub-index $\infty$. See also Definition 5.7

5.4. Angular sectors on a Riemann surface. We enlarge Definition 5.2 for an arbitrary Riemann surface.

Definition 5.7. A singular analytic vector field $X$ on $M$ with an isolated singularity at $q \in M$, has a center, hyperbolic, elliptic, parabolic or class 1 entire angular sector at $q$, if there exist a germ of angular sector $(\mathcal{A}, q) \subset M$ and a biholomorphism

$$
\Upsilon:((\mathcal{A}, q), X) \longrightarrow W, \quad W \in\left\{C_{2 \pi / \lambda_{2}}, H, E, P_{\nu}, \mathscr{E}\right\} \text { respectively }
$$


making both germs of vector fields holomorphically equivalent. See Figure 4 ,

Philosophically, several remarks regarding this definition are in order:

i) Obviously the center case $C_{2 \pi / \lambda_{2}}$ is useful, though strictly speaking, it is not a topological angular sector.

ii) Note that $\Upsilon$ in Definition 5.7 is an analytic equivalence of vector fields; hence the respective (real) time behaviour of the trajectories of $((\mathcal{A}, q), X)$ and $W$ must coincide.

For example, if we consider a parabolic sector $P_{\nu}$, $\mathfrak{I m}(\nu)<0$, having all the trajectories defined for positive time $\left(t_{0}, \infty\right)$, then the same holds for $((\mathcal{A}, q), X)$ when $\Upsilon$ exists.

Remark 5.8. It is to be noted that many other types of angular sectors are not yet considered. For instance, the vector field germs determined by

$$
X_{1}(z)=2(\cosh (z)+1) \frac{\partial}{\partial z}, \quad X_{2}(z)=\tan (z) \frac{\partial}{\partial z} \quad \text { on } M=\widehat{\mathbb{C}},
$$

centred at $q=\infty \in \widehat{\mathbb{C}}$, have an accumulation of poles and zeros.

Example 5.9 (Example 3.10 revisited). The class 2 essential singularity of the vector field

$$
X_{3}(z)=\mathrm{e}^{z} \mathrm{e}^{-\mathrm{e}^{z}} \frac{\partial}{\partial z}
$$

at $\infty \in \widehat{\mathbb{C}}$ which does not have an accumulation point of poles or zeros, determines a new type of angular sector $\mathscr{E}^{\mathscr{E}}$. Noticing that there is a reflection symmetry (of $\mathfrak{R e}(X))$ about the real axis, it is enough to consider the half-plane $\left(\overline{\mathbb{H}}_{+}, \infty\right) \subset \widehat{\mathbb{C}}_{z}$, which is in turn composed of an "infinite number of nested class 1 entire sectors" $\mathscr{E}_{k}, k \geq 1$. See Figures 2 and 5 .

For the vector fields of Remark 5.8 and Example 5.9, the finite decomposition, as sectors $H, E, P_{\nu}, \mathscr{E}$ (following the spirit of Theorem 5.1) on some disk $V_{j} \subset \widehat{\mathbb{C}}$ centerd at $q=\infty$, does not apply.

5.5. Angular sectors in the flat surfaces category. It will be very useful to think about angular sectors, $C_{2 \pi / \lambda_{2}}, H, E, P_{\nu}, \mathscr{E}$ of a germ $((\mathcal{A}, q), X)$ on $M$, as germs of singular flat surfaces. In order to describe this accurately, we assume Definition 5.7, whence the composition

$$
\Psi_{X}=\Psi_{X_{\iota}} \circ \Upsilon:\left((\mathcal{A}, q), g_{X}\right) \subset M \longrightarrow\left(\left(\mathcal{A}_{0}, q_{0}\right), g_{X_{\iota}}\right) \longrightarrow\left(\mathbb{C}_{t}, \delta\right)
$$

(here $X_{\iota}$ comes from $C_{2 \pi / \lambda_{2}}, H, E, P_{\nu}, \mathscr{E}$, see Definition [5.2) is an orientation preserving local isometry. We enrich the conditions of \$5.3, adding the following metric properties.

Remark 5.10 (Metric attributes of the angular sectors). Let $\left((\mathcal{A}, q), g_{X}\right) \subset M$ be an angular sector of kind $C_{2 \pi / \lambda_{2}}, H, E, P_{\nu}, \mathscr{E}$ as in Definition 5.7

1. The trajectories $\sigma_{1}, \sigma_{2}$ in the boundary $\partial \mathcal{A}=\{q\} \cup \sigma_{1} \cup \gamma \cup \sigma_{2}$ are geodesics in $\left(M, g_{X}\right)$, having well-defined finite or infinite $g_{X}$-length (which coincide with the real time in that $\sigma_{\iota}$ arrives at $q$, or escapes from $q$, recall equation (2.5)).

2. The path $\Gamma=\Psi_{X}(\gamma)$ in $\mathbb{C}_{t}$ is the image of $\gamma$. 


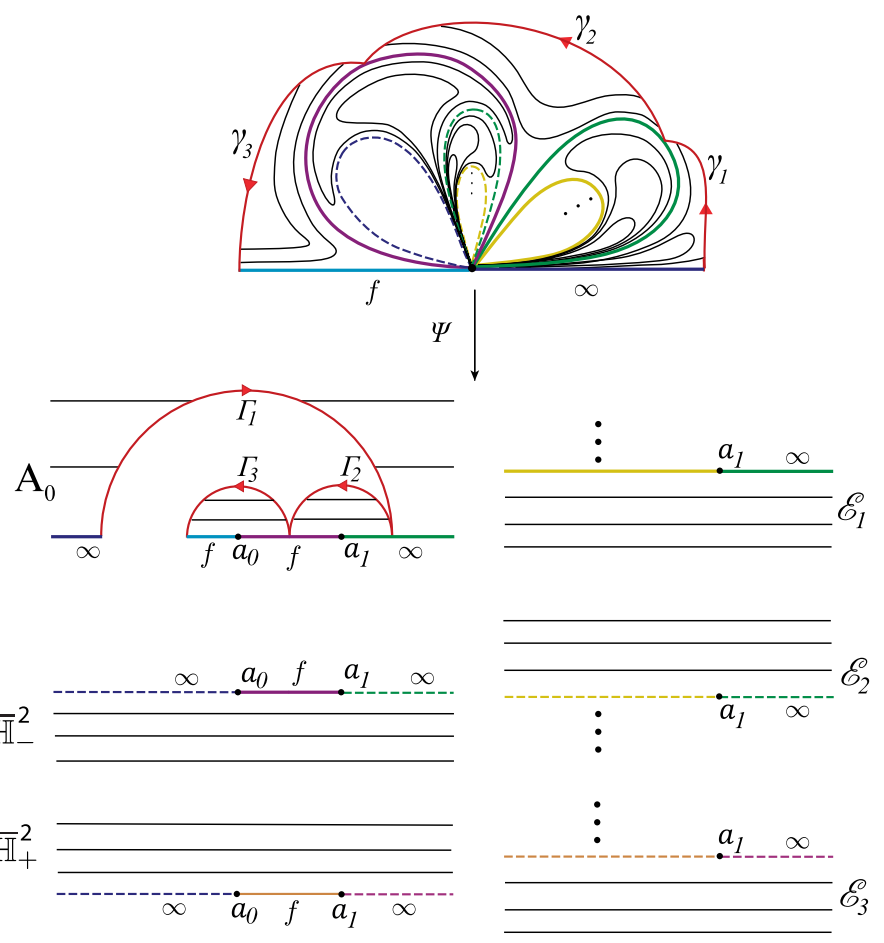

Figure 5. Class 2 entire sector. Top drawing shows the sector $\mathscr{E} \mathscr{E}=\left(\left(\overline{\mathbb{H}}^{2}, \infty\right), \mathrm{e}^{z} \mathrm{e}^{-\mathrm{e}^{z}} \frac{\partial}{\partial z}\right)$, while the bottom drawing describes the image $\Psi(\mathcal{A})$. The path $\gamma=\gamma_{1} \cup \gamma_{2} \cup \gamma_{3}$ is chosen so that its image $\Psi\left(\gamma_{1} \cup \gamma_{2} \cup \gamma_{3}\right)=\Gamma_{1} \cup \Gamma_{2} \cup \Gamma_{3}$ determines three semicircles and such that $\Gamma$ bounces off the horizontal trajectory that contains the finite asymptotic values $a_{0}$ and $a_{1}$. In $\mathcal{A}$ : the region between the boundary of the sector (dark blue, red and light blue trajectories) and the green and purple paths, correspond to the region denoted by $A_{0}$ in the image of $\Psi$. Furthermore, the region delimited by the purple path is itself a "copy" of the whole class 2 entire sector. In $\Psi(\mathcal{A})$ : like colored segments are glued together. The green and yellow segments enclose a half-plane, which determines a half-logarithmic spiral related to a class 1 entire sector associated to the asymptotic value $a_{1}$ (denoted by $\mathscr{E}_{1}$ ). The region below the purple segment corresponds to the half-plane $\overline{\mathbb{H}}_{-}^{2}$ that is glued to $A_{0}$ precisely by the purple segment. On the half-plane $\overline{\mathbb{H}}_{-}^{2}$ (and $\overline{\mathbb{H}}_{+}^{2}$ ) there is a copy of the asymptotic value $a_{1}$, giving rise to a half-logarithmic spiral related to a class 1 entire sectors associated to the copy of $a_{1}$ denoted by $\mathscr{E}_{2}\left(\mathscr{E}_{3}\right.$ for $\left.\overline{\mathbb{H}}_{+}^{2}\right)$. The whole construction is then repeated for $\overline{\mathbb{H}}_{+}^{2}$ by glueing the dotted dark blue segments of $\overline{\mathbb{H}}_{-}^{2}$ and $\overline{\mathbb{H}}_{+}^{2}$. This process is repeated ad infinitum $\left(\overline{\mathbb{H}}_{+}^{2}\right.$ and $\mathscr{E}_{3}$ are not drawn in $\left.\mathcal{A}\right)$. See Examples 5.9 and 5.12 . 
5.5.1. Flat metrics on centers $C_{2 \pi / \lambda_{2}}$. We assume that the vector field germ representative $((\mathcal{A}, q), X)$ on $M$ is a center $C_{2 \pi / \lambda_{2}}$. We describe the case $\lambda_{2}>0\left(\lambda_{2}<0\right.$ is analogous). Let $\sigma$ be a trajectory of $\mathfrak{I m}(X)$ in $\mathcal{A}$. The distinguished parameter

$$
\Psi_{X}: \mathcal{A} \backslash(\{q\} \cup \sigma) \subset M \longrightarrow\left(0,2 \pi /\left|\lambda_{2}\right|\right) \times i(0, \infty) \subset \mathbb{C}_{t}
$$

is a univalued map. Obviously, the strip on the right gives origin to a flat semiinfinite height cylinder $S_{2 \pi /\left|\lambda_{2}\right|}^{1} \times(0, \infty)$, of perimeter $2 \pi /\left|\lambda_{2}\right|$ (or semi-infinite height annulus flow 8$)$. The $\lim _{p \rightarrow q} \Psi_{X}(p)=\infty \in \widehat{\mathbb{C}}_{t}$ corresponds to the Riemannian end of the cylinder.

$2 \pi /\left|\lambda_{2}\right| \in \mathbb{R}^{+}$is a complete isometric invariant of $C_{2 \pi / \lambda_{2}}$.

5.5.2. Flat metrics on hyperbolic sectors $H$. The germ of vector field $X$ on $M$ has a hyperbolic sector $H$ at a singularity $q \in M$, when a map $\Psi_{X}: \mathcal{A} \subset M \rightarrow \mathbb{C}_{t}$ satisfying the following conditions exists:

$\Psi_{X}(q)=a \in \mathbb{C}_{t}$.

Both geodesics $\sigma_{1}, \sigma_{2} \subset \partial \mathcal{A}$ of $\mathfrak{R e}(X)$ arrive at or escape from $q$, in a finite real time $\tau>0$, this is noted in Figure 4 .

$\Psi_{X}(\mathcal{A})$ is an upper or lower half-disk, and $\Psi_{X}\left(\sigma_{1} \cup \sigma_{2}\right)$ is in a horizontal trajectory segment, Figure 4 shows the case of a upper half-disk.

The metric $g_{X}$ is real analytic at $q$ in $\mathcal{A}$.

5.5.3. Flat metrics on elliptic sectors $E$. The respective map $\Psi_{X}: \mathcal{A} \subset M \rightarrow \widehat{\mathbb{C}}_{t}$ satisfies:

$\Psi_{X}(q)=\infty \in \widehat{\mathbb{C}}_{t}$.

All the geodesics (including the characteristic trajectories $\left.\sigma_{1}, \sigma_{2} \subset \partial \mathcal{A}\right)$ of $\mathfrak{R e}(X)$ arrive at or escape (both possibilities occur in this sector) from $q$ having infinite $g_{X}$-length, this is noted in Figure 4.

$\Psi_{X}(\mathcal{A})$ is contained in an upper or lower half-plane, and $\Psi_{X}\left(\sigma_{1} \cup \sigma_{2}\right)$ are two semi-rays in a horizontal trajectory, Figure 4 shows the case of a upper half-plane minus a half-disk.

5.5.4. Flat metrics on parabolic sectors $P_{\nu}$. Recall that $\nu=\nu_{1}+i \nu_{2} \in \mathbb{C} \backslash \mathbb{R}$. The respective map $\Psi_{X}: \mathcal{A} \subset M \rightarrow \widehat{\mathbb{C}}_{t}$ satisfies:

$\Psi_{X}(q)=\infty \in \widehat{\mathbb{C}}_{t}$.

All the geodesics, $\sigma_{1}, \sigma_{2} \subset \partial \mathcal{A}$ of $\mathfrak{R e}(X)$ arrive at or escape (only one possibility occurs) from $q$, having infinite $g_{X}$-length, this is noted in Figure 4 .

$\Psi_{X}(\mathcal{A})$ is a left (or right) strip and $\Psi_{X}\left(\sigma_{2} \cup \sigma_{2}\right)$ are two horizontal half-rays, Figure 4 shows the case of a left strip.

The height $\mathfrak{I m}(\nu)=\nu_{2} \neq 0$ is an isometric invariant of the flat surface associated to $P_{\nu}$. Moreover, the complete $\nu \in \mathbb{C} \backslash \mathbb{R}$ will be useful for us (in Table 2).

5.5.5. Flat metrics on class 1 entire sectors $\mathscr{E}$. Let $((\mathcal{A}, q), X)$ be the a vector field germ of a class 1 entire sector on $M$. We assume that $\Upsilon(\mathcal{A})$ is $\mathscr{E}=\left(\left(\overline{\mathbb{H}}_{+}^{2}, \infty\right), \mathrm{e}^{z} \frac{\partial}{\partial z}\right)$, in the upper half-plane, for the lower half-plane, details change in an obvious way. The distinguished parameter $\Psi_{X}$ of $\mathscr{E}$ is chosen as in (5.1). The $\lim _{z \rightarrow q} \Psi_{X}(z)=$ $a, \infty \in \widehat{\mathbb{C}}_{t}$ are the two asymptotic values of $\Psi_{X}$, associated with $q$, recall (5.3).

The boundary geodesics $\sigma_{1}, \sigma_{2} \subset \partial \mathcal{A}$ of $\mathfrak{R e}(X)$ in $M$ have finite and infinite $g_{X}$-length (5.2), this is noted in Figure 3 .

\footnotetext{
${ }^{8}$ This notation follows the classical concepts of L. Markus; see 47 and Table 3 in 11 Appendix for more details.
} 
$\Psi_{X}(\mathcal{A})$ determines a half-logarithmic spiral and $\Psi_{X}\left(\sigma_{1} \cup \sigma_{2}\right)$ is in a horizontal trajectory, once again refer to Figure 3 .

5.6. Isometric glueing. In the flat surface category, surgery tools are widely used; e.g. 66 pp. 56 "welding of surfaces", 49], 44, or 69. Let us recall a concrete advantage of this point of view:

Corollary 5.11 (Isometric glueing). Let $\left(M^{0}, g_{X}\right),\left(N^{0}, g_{Y}\right)$ be two flat surfaces arising from two singular complex analytic vector fields $X$ and $Y$. Assume that both spaces $M^{0}, N^{0}$ have as geodesic boundary components of the same length: the trajectories $\sigma_{1}, \sigma_{2}$ of $X$ and $Y$. The isometric glueing of them along these geodesic boundary, is well defined, and provides a new flat surface on $M^{0} \cup N^{0}$ arising from a new complex analytic vector field.

Proof. The foliation by geodesics $\mathfrak{R e}(X)$ on $\left(M^{0}, g_{X}\right)$ is a translation structure (in the sense of geometric structures following W. Thurston 69] section 3.3, pp. 125); having $\left\{z \mapsto z+a_{\text {jk }}\right\}$ as a change of coordinates. The same applies for $\mathfrak{R e}(Y)$ on $\left(N^{0}, g_{Y}\right)$. The geodesic boundaries $\sigma_{1}, \sigma_{2}$ are glued via an orientation preserving isometry (the trajectory time orientations must match), which gives rise to a new flat surface $M^{0} \cup N^{0}$ having a translation structure. Using Lemma 2.6, the existence of a complex analytic vector field on $M^{0} \cup N^{0}$ extending $X$ and $Y$ follows.

Alternatively the proof may be obtained as a simple application of the reflection principle, 2] pp. 172 .

The isometric glueing Corollary 5.11 has already been applied several times for the construction of angular sectors, in particular, the class 1 entire sectors in $\$ 5.3 .1$ and class 2 entire sectors in Example 5.9.

Note that in general when glueing geodesic boundaries $\sigma_{1}, \sigma_{2}$ with endpoints there is no control as to what type of singularity one might have at the endpoints of the geodesic boundaries. However, in the case of angular sectors we will obtain a conformal puncture; see Proposition 5.20.

Example 5.12 (Example 3.10 revisited). Considering as starting point the class 2 entire sector $\mathscr{E}^{\mathscr{E}}$, note that the complexity of the flat metric attributes is greatly increased: it is possible to construct an infinite number of conformally different class 2 entire sectors. In Figure 5, on each nested copy, $\mathscr{E}_{k}$, of a class 1 entire sector, there is associated a finite asymptotic value $a_{k} \in \mathbb{C}_{t}$ and a copy of the asymptotic value $\infty$. Thus, the asymptotic value $\infty$ has infinite multiplicity. Moreover, the $a_{k} \in \mathbb{C}_{t}$ could all be different, so in fact each infinite sequence $\left(a_{0}, a_{1}, a_{2}, \ldots\right)$ of finite asymptotic values, with $a_{0} \neq a_{k}$ for $k \geq 1$, defines a conformally different class 2 entire angular sector. A deeper study of these cases is left as a subject of future work.

Example 5.13 (Differences between real and complex analytic angular sectors). Let $Z=x^{2} \frac{\partial}{\partial x}-y \frac{\partial}{\partial y}$ be a real analytic saddle-node on $\left(\mathbb{R}^{2}, \overline{0}\right)$. Following Theorem 5.1, at the origin, its singular planar real analytic foliation $\mathcal{F}$ has two hyperbolic sectors $Z_{h}$ and one parabolic sector $Z_{p}$.

The hyperbolic angular sectors of $Z$ at $q=0$ can not be recognized with our flat sectors $H$ (since the maximal interval of time definition for the characteristic trajectories of $Z$ assumes $\left(\tau_{0}, \infty\right)$ or $\left(\infty, \tau_{0}\right)$ at $\left.\overline{0}\right)$.

G. León-Gil et al. [45, show that there is no complex structure $J$, making the real analytic vector field germ $\left(\left(\mathbb{R}^{2}, \overline{0}\right), Z\right)$, the real part of the complex analytic 
vector field $X$. That is, for any choice of Riemann surface germ $\left(\left(\mathbb{R}^{2}, \overline{0}\right), J\right)$ and any choice of singular complex analytic vector field $X$ on each Riemann surface germ, the equation $\mathfrak{R e}(X)=Z$ is not satisfied.

5.7. Cyclic words $\mathcal{W}_{X}$ at poles and zeros. We recall the analytic normal forms at poles and zeros of germs $((\mathbb{C}, 0), X)$ whose complete analytic invariants are the order and the residue. See Table 2, proved in [39] ch. 3, 26], 27, 1] pp. 111, 14, [66] ch. III, 24]. Our goal is to recognize the cyclic words which are determined. We use as a main parameter $\lambda \in \mathbb{C}^{*}$ in the normal forms, from this we obtain the respective residue and the metric invariants $\left(2 \pi / \lambda_{2}, 2 \pi i / \lambda,-2 \pi \lambda_{2}\right.$ and $\left.2 \pi i \lambda\right)$.

Thus it will be convenient to introduce a new letter $E_{\nu}$, indicating a $\nu$ surgery, as will be explained in case 5 (note that $E=E_{0}$ so in fact $E_{\nu}$ replaces $E$ ). See also Remark 5.17] and Proposition 5.20.

TABle 2. Poles and zeros $\left(\lambda=\lambda_{1}+i \lambda_{2} \in \mathbb{C}^{*}\right)$

\begin{tabular}{|c|c|c|c|c|}
\hline $\begin{array}{l}\text { normal form } \\
\text { of } X\end{array}$ & $\begin{array}{l}\text { order } \\
\text { of } X\end{array}$ & $\begin{array}{l}\text { residue } \\
\text { of } \omega_{X}\end{array}$ & $\begin{array}{c}\text { metric } \\
g_{X} \\
\end{array}$ & $\begin{array}{l}\text { cyclic } \\
\text { word }\end{array}$ \\
\hline$\frac{1}{z^{k}} \frac{\partial}{\partial z}$ & $-k \leq-1$ & 0 & $\begin{array}{c}\text { cone angle } \\
(2 k+2) \pi\end{array}$ & $\underbrace{H \cdots H}_{2 k+2}$ \\
\hline$i \lambda_{2} z \frac{\partial}{\partial z}$ & \multirow{2}{*}{$s=1$} & $\frac{1}{i \lambda_{2}} \in i \mathbb{R}^{*}$ & $\begin{array}{c}\text { cylinder } \\
S_{2 \pi /\left|\lambda_{2}\right|}^{1} \times(0, \infty)\end{array}$ & $C_{2 \pi / \lambda_{2}}$ \\
\hline$\lambda z \frac{\partial}{\partial z}$ & & $\frac{1}{\lambda} \in \mathbb{C} \backslash i \mathbb{R}$ & $\begin{array}{c}\text { cylinder } \\
S_{2 \pi\left|\lambda_{1}\right| /|\lambda|}^{1} \times(0, \infty)\end{array}$ & $P_{2 \pi i / \lambda}$ \\
\hline$z^{s} \frac{\partial}{\partial z}$ & \multirow{3}{*}{$s \geq 2$} & 0 & $(2 s-2)$ copies $\left(\overline{\mathbb{H}}^{2}, \infty\right)$ & $\underbrace{E \cdots E}_{2 s-2}$ \\
\hline$\frac{z^{s}}{1+\lambda_{2} z^{s-1}} \frac{\partial}{\partial z}$ & & $i \lambda_{2} \in i \mathbb{R}^{*}$ & $\begin{array}{c}(2 s-2) \text { copies }\left(\overline{\mathbb{H}}^{2}, \infty\right) \\
\text { with }-2 \pi \lambda_{2} \text { surgery }\end{array}$ & $\underbrace{E \cdots E}_{2 s-3} E_{-2 \pi \lambda_{2}}$ \\
\hline$\frac{z^{s}}{1+\lambda z^{s-1}} \frac{\partial}{\partial z}$ & & $\lambda \in \mathbb{C} \backslash i \mathbb{R}$ & $\begin{array}{c}(2 s-2) \text { copies }\left(\overline{\mathbb{H}}^{2}, \infty\right) \\
\text { and a strip } \\
\text { with } 2 \pi i \lambda \text { surgery }\end{array}$ & $\underbrace{E \cdots E}_{2 s-2} P_{2 \pi i \lambda}$ \\
\hline
\end{tabular}

From the normal form $((\mathbb{C}, 0), X)$, first column Table 2 , to the flat metric $((\mathbb{C}, 0)$, $\left.g_{X}\right)$. We work in a open disk $D(0,2 \epsilon)$ having radius $2 \varepsilon>0$ small enough, thus

$$
\Psi(z)=\int_{\varepsilon}^{z} \omega_{X}: D(0, \varepsilon) \backslash \sigma \subset \mathbb{C}_{z} \longrightarrow \mathbb{C}_{t}
$$

and $\Psi(\epsilon)=0$. Moreover, in order to get a univalued $\Psi$, it is convenient to remove a path $\sigma$ between 0 and $\epsilon$ from $D(0, \epsilon)$. There are three possible choices.

a) Zeros of order $s=1$ and $\lambda_{1}=0$; the trace of $\sigma$ is inside the ray $\mathbb{R}^{+} \subset \mathbb{C}_{z}$; see Figure 6. a and d.

b) Zeros of order $s=1, \lambda_{1} \neq 0 ; \sigma$ is the trajectory of $X$ with initial condition $\epsilon=\sigma(0)$, it is a spiral trajectory; see Figure 6. b, c and e. 
c) Zeros of order $s \geq 2 ; \sigma$ is the trajectory of $X$ with initial condition $\epsilon=\sigma(0)$, the trace of $\sigma$ is inside the ray $\mathbb{R}^{+} \subset \mathbb{C}_{z}$; see Figure 7

In Table 2, for the case of zeros, the displacement of $\gamma$ or $2 \pi i$ times the residue of $\omega_{X}$ is

$$
\int_{\gamma} \omega_{X}= \begin{cases}\frac{2 \pi i}{\lambda}=\frac{2 \pi\left(\lambda_{2}+i \lambda_{1}\right)}{\lambda_{1}^{2}+\lambda_{2}^{2}} & s=1, \\ 2 \pi i \lambda=2 \pi\left(-\lambda_{2}+i \lambda_{1}\right) & s \geq 2,\end{cases}
$$

here $\gamma(\theta)=\varepsilon \mathrm{e}^{i \theta}:[0,2 \pi] \rightarrow(\mathbb{C}, 0)$ is an oriented circle, whose radius $\varepsilon$ is small enough. Note that (15.6) is also the complex time of $\gamma$, which is the natural generalization of (2.4). Hence the displacement makes sense for the paths $\gamma \subset \partial \mathcal{A}$, as in Remark 5.10. This displacement gives origin to the surgeries in Table 2, as we will show below.

Following ideas of quadratic differentials [66] ch. VII, the height of $\Gamma$ is defined as

$$
\left|\mathfrak{I m}\left(\int_{\gamma} \omega_{X}\right)\right|=\left\{\begin{array}{cl}
\frac{2 \pi\left|\lambda_{1}\right|}{|\lambda|^{2}} & s=1, \quad \lambda=\lambda_{1}+i \lambda_{2} \in \mathbb{C} \backslash i \mathbb{R}, \\
2 \pi\left|\lambda_{1}\right| & s \geq 2 .
\end{array}\right.
$$

Case 1. A pole.

Let $\left((\mathbb{C}, 0), \frac{1}{z^{k}} \frac{\partial}{\partial z}\right)$ be the vector field germ, then the distinguished parameter is

$$
\Psi(z)=\int_{0}^{z} \zeta^{k} d \zeta=\frac{z^{k+1}}{k+1}: D(0, \varepsilon) \longrightarrow \mathbb{C}_{t} .
$$

The associated word is $H \cdots H$, having $2+2 k$ letters.

The cone angle $(2+2 k) \pi$ of $g_{X}$ characterizes the order $-k$ of the pole9, thus the cone angle is a complete metric and analytic invariant of the germ; see 48, pp. 1028.

Case 2. A simple zero with pure imaginary linear part $i \lambda_{2} \in i \mathbb{R}^{*}$.

Let $\left((\mathbb{C}, 0), i \lambda_{2} z \frac{\partial}{\partial z}\right)$ be the vector field germ, then

$$
\Psi(z)=\int_{\epsilon} \frac{d \zeta}{i \lambda_{2} \zeta}: D(0, \epsilon) \backslash\{[0, \epsilon]\} \longrightarrow\left(0,2 \pi / \lambda_{2}\right) \times i(0, \infty) \subset \mathbb{C}_{t}, \quad \epsilon \in \mathbb{R}^{+} ;
$$

see Figure 6] for $\lambda_{2}>0$. The metric $g_{X}$ on $D(0, \epsilon)$ can be described as a semiinfinite height cylinder of perimeter $2 \pi /\left|\lambda_{2}\right|$; recall [5.5.1. The case $\lambda_{2}<0$ is analogous, Figure 6]. The associated word is $C_{2 \pi / \lambda_{2}}$.

Case 3. A simple zero with linear part $\lambda=\lambda_{1}+i \lambda_{2} \in \mathbb{C} \backslash i \mathbb{R}$.

Let $\left((\mathbb{C}, 0), \lambda z \frac{\partial}{\partial z}\right)$ be the vector field germ. Assume that $\lambda_{1}>0$, then 0 is a source for the trajectories of $X$. Moreover, the image $\Psi(D(0, \epsilon) \backslash \sigma)$ is the interior of a left horizontal strip $S_{2 \pi i / \lambda} \subset \mathbb{C}_{t}$ having vertical height $2 \pi\left|\lambda_{1}\right| /|\lambda|^{2}$; see Figure 6], b, c for two examples. $S_{2 \pi i \lambda}$ has boundary the straight line segment $\overline{02 \pi i / \lambda}$ and two horizontal left half-rays.

If $\lambda_{1}<0$, then 0 is a sink for the trajectories of $X$. We use a right horizontal strip $S_{2 \pi i / \lambda}$ in a manner analogous as above, Figure 6. provides an example.

\footnotetext{
${ }^{9} \mathrm{We}$ convene that the order (or multiplicity) of a pole is to be negative.
} 
a)

$$
i z \frac{\partial}{\partial z}
$$
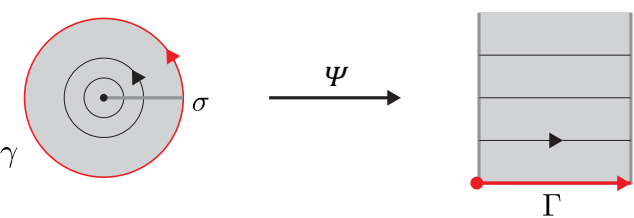

b)

$$
\begin{gathered}
\left(\lambda_{1}+i \lambda_{2}\right) z \frac{\partial}{\partial z} \\
\lambda_{1}, \lambda_{2}>0
\end{gathered}
$$
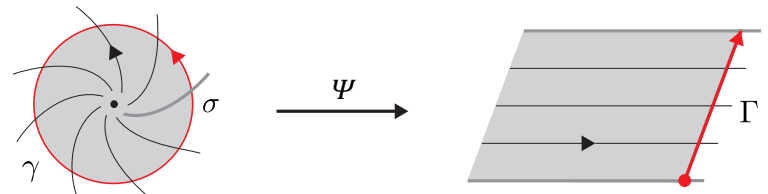

c)

$$
z \frac{\partial}{\partial z}
$$
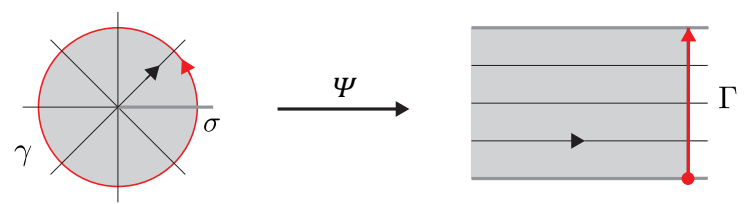

d)

$$
-i z \frac{\partial}{\partial z}
$$
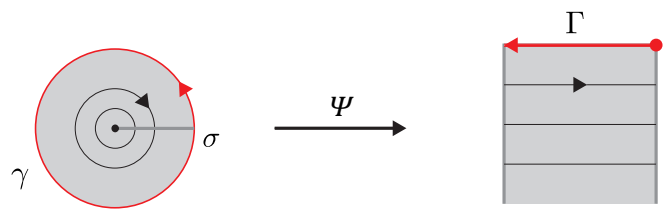

e)

$$
-z \frac{\partial}{\partial z}
$$
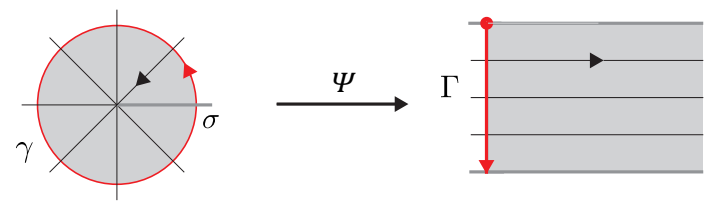

FiguRE 6 . Let $X(z)=\lambda z \frac{\partial}{\partial z}$ be a vector field having a simple zero, $\lambda \in \mathbb{C}^{*}$, we consider the displacement $\int_{\gamma} \omega_{X}=2 \pi i / \lambda$ as the red arrow in right drawings. In all the cases, the metric $g_{X}$ determines a semi-infinite cylinder $S_{\rho}^{1} \times(0, \infty)$, where $\rho$ is the perimeter (see Table 2), which can be obtained by suitable identification of a strip in $\mathbb{C}_{t}$. In (b), (c) and (e), $\sigma \subset \mathbb{R}^{+}$is a characteristic trajectory, having well-defined slope at 0 only for $\lambda_{2}=0$. See $\$ 5.7$.

A characteristic trajectory $\sigma$ of $X$ with well-defined slope exists if and only if $\lambda_{2}=0$. When $\lambda_{2} \neq 0$, we use a trajectory $\sigma$ of $X$ in order to cut the disk $D(0, \epsilon)$, even if this trajectory $\sigma$ behaves as a spiral at 0 .

Using the strip $S_{2 \pi i / \lambda} \subset \mathbb{C}_{t}$ we can recover a semi-infinite height cylinder as a flat surface $g_{X}$; by glueing between them the two horizontal boundary components using the isometric glueing $t \leftrightarrow t+(2 \pi i / \lambda)$. The perimeter of the cylinder is $2 \pi\left|\lambda_{1}\right| /|\lambda|^{2}$. The associated word is $P_{2 \pi i / \lambda}$. 


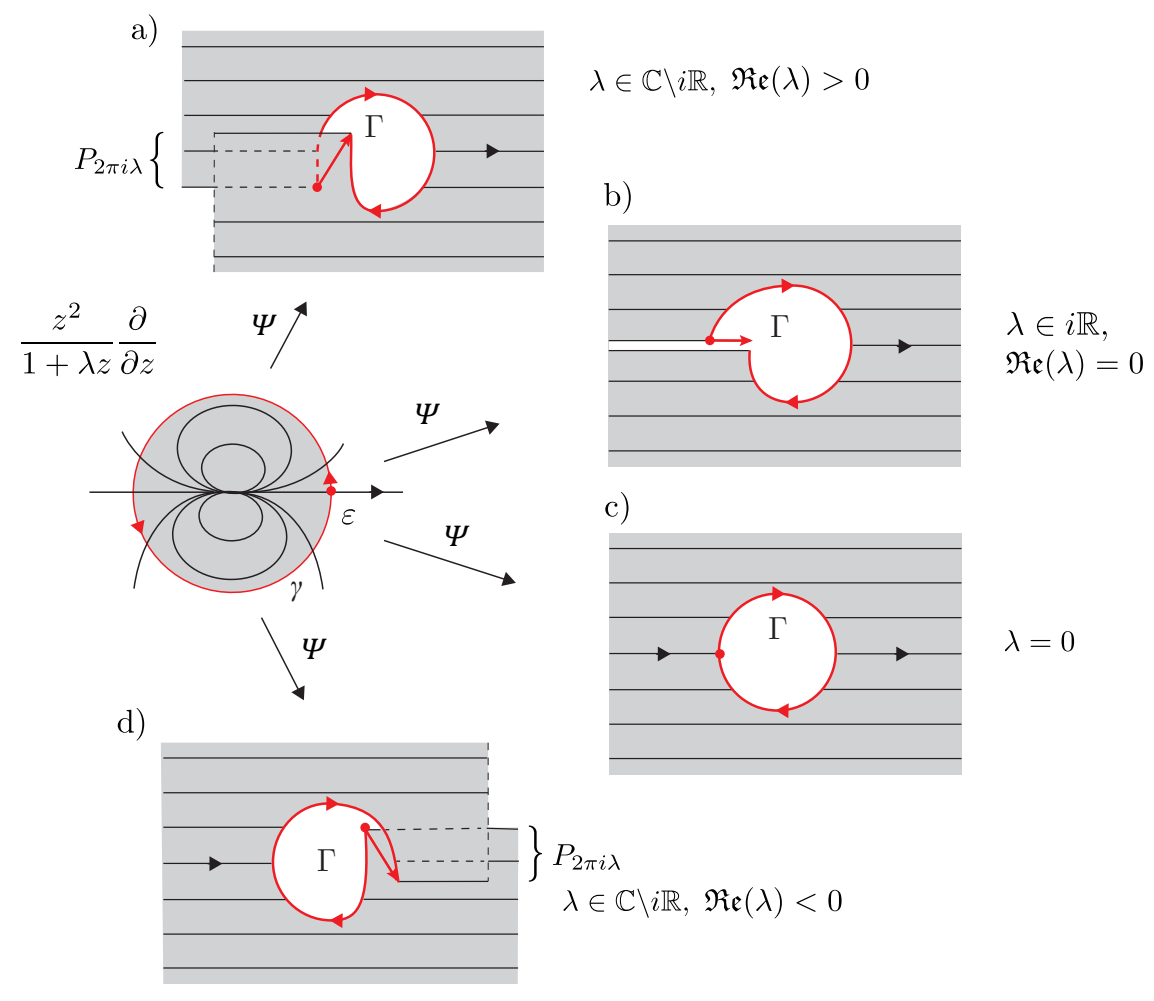

Figure 7. Let $X(z)=\frac{z^{2}}{1+\lambda z} \frac{\partial}{\partial z}$ be a vector field having a double zero, $\lambda \in \mathbb{C}$. When $\lambda \neq 0$, we consider the displacement $\int_{\gamma} \omega_{X}=2 \pi i \lambda$ as the red arrow on the right drawings. a) $\mathfrak{I m}(2 \pi i \lambda)>0$ produces two elliptic sectors and one parabolic sector. b) $\mathfrak{I m}\left(2 \pi i \lambda_{2}\right)=0$ produces two elliptic sectors, the $-2 \pi \lambda_{2}$ surgery is a glueing along the horizontal boundary, it is given by an euclidean translation $\left\{t \leftrightarrow t-2 \pi \lambda_{2}\right\}$ as in Corollary 5.11, c) $\lambda=0$ produces two elliptic sectors. d) $\mathfrak{I m}(2 \pi i \lambda)<0$ produces two elliptic sectors and one parabolic sector. In all cases, $\sigma=[0, \epsilon] \subset \mathbb{R}^{+}$ in $\mathbb{C}_{z}$ is a characteristic trajectory of $X$. See $\$ 5.7$.

Case 4. A zero of order $s \geq 2$ having zero residue.

Let $\left((\mathbb{C}, 0), z^{s} \frac{\partial}{\partial z}\right)$ be the vector field germ. Assume by simplicity $s=2$, let

$$
\Psi(z)=\int_{\epsilon}^{z} \frac{d \zeta}{\zeta^{2}}=-\frac{1}{z}+\frac{1}{\epsilon}: D(0, \epsilon) \backslash \sigma \longrightarrow \widehat{\mathbb{C}}_{t}, \quad \epsilon \in \mathbb{R}^{+},
$$

be the global distinguished parameter, satisfying $\Psi(\epsilon)=0$. Since the path $\gamma$ is a small circle enclosing $z=0$, the path $\Gamma$ closes itself having clockwise orientation. In case $s=2$; see Figure 7.c. Two elliptic sectors $E$ are defined by the intersection of horizontal trajectory in $\widehat{\mathbb{C}}_{t}$ and the image of $\Psi . g_{X}$ is isometric to the euclidean metric of $\left(\widehat{\mathbb{C}}_{t}, \delta\right)$ at $\infty$. The associated word is $E E$. 
If $\lambda=0$ and $s \geq 3$, the obvious glueing applies for $t^{2} \frac{\partial}{\partial t}$ arising as the pullback via $t: z \mapsto z^{s-1}$. The resulting $g_{X}$ is a ramified covering of the euclidean metric of $\left(\widehat{\mathbb{C}}_{t}, \delta\right)$ at $\infty$ with $(s-1)$ ramification index. In this case $2 s-2$ elliptic sectors $E$ are present. The path $\Gamma$ closes itself having clockwise orientation. We obtain the associated word $E \cdots E$, having $2 s-2$ letters.

Case 5. A zero of order $s \geq 2$ having residue $i \lambda_{2} \in i \mathbb{R}^{*}$.

Let $\left((\mathbb{C}, 0), \frac{z^{s}}{1+\lambda_{2} z^{s-1}} \frac{\partial}{\partial z}\right)$ be the vector field germ. We take a characteristic trajectory $\sigma$ of $z^{s} \frac{\partial}{\partial z}$ that connects 0 to $\epsilon \in \mathbb{R}$ in $\left((\mathbb{C}, 0), z^{s} \frac{\partial}{\partial z}\right)$, as in case 4 . Then one performs the following $2 \pi \lambda_{2}$ surgery on said trajectory: cut along the trajectory $\overline{0 \epsilon}$ and glue it back together with a horizontal shift by $2 \pi \lambda_{2}$ in $g_{X}$-units. This adds a real residue to the singularity at $z=0$, we obtain the germ $\left((\mathbb{C}, 0),\left(z^{s} /\left(1+i \lambda_{2}^{s-1}\right)\right) \frac{\partial}{\partial z}\right)$. The respective $\Psi: D(0, \epsilon) \backslash \sigma \subset \mathbb{C}_{z} \longrightarrow \mathbb{C}_{t}$ is in Figure [7.b. No parabolic sectors appear. Furthermore, the path $\Gamma$ does not close itself: it is missing a strictly real segment corresponding to the displacement $-2 \pi \lambda_{2} \in \mathbb{R}^{*}$. The associated word is

$$
E \cdots E E_{\nu}
$$

having $2 s-3$ letters $E$ with the introduction of the new letter $E_{\nu}$, where we recall that we have a $\nu=-2 \pi \lambda_{2} \in \mathbb{R}^{*}$ surgery on $E$ to obtain this new letter $E_{\nu}$.

Case 6 . A zero of order $s \geq 2$ having residue $\lambda=\lambda_{1}+i \lambda_{2} \in \mathbb{C} \backslash i \mathbb{R}$.

Let $\left((\mathbb{C}, 0), \frac{z^{s}}{1+\lambda z^{s-1}} \frac{\partial}{\partial z}\right)$ be the vector field germ. For pure imaginary $i \lambda_{2}$, we take an imaginary trajectory of $\left((\mathbb{C}, 0), z^{s} \frac{\partial}{\partial z}\right)$ that connects $i \epsilon$ and 0 . Then one performs the surgery with a displacement of $2 \pi i \lambda$ in $g_{X}$-units. Again this adds an imaginary residue to $z=0$. Once again $2 s-2$ elliptic sectors $E$ appear, and a parabolic sector $P_{2 \pi i \lambda}$, appears. See Figure 7. (a) and (d). The path $\Gamma$ does not close itself: the displacement corresponds to $2 \pi i$ times the residue $\lambda$. The associated word is

$$
E \cdots E P_{2 \pi i \lambda}
$$

having $2 s-2$ letters $E$ and one letter $P_{2 \pi i \lambda}$.

5.8. Angular sectors of vector fields in the combinatorial category. We now re-examine vector fields from quite a different perspective. Let $\left(\left(\mathbb{R}^{2}, \overline{0}\right), Z\right)$ be a real analytic vector field germ as in Theorem 5.1 then we have a correspondence from vector fields to words

$$
Z \longmapsto \mathcal{W}_{Z}=W_{1} \cdots W_{k}, \quad W_{\iota} \text { in the } Z_{h}, Z_{e}, Z_{p} \text { alphabet. }
$$

The three real analytic angular sectors in the alphabet on the right-hand side of (5.10), are defined in \$5.1. By simplicity, we are looking at these angular sectors as objects in the $C^{\infty}$ smooth category or even simpler in the combinatorial category. Thus, from now on, we shall also consider

$$
C_{2 \pi / \lambda_{2}}, H, E_{\nu}, P_{\nu}, \mathscr{E}_{\nu}
$$

as "letters in an alphabet".

Here, $\mathscr{E}_{\nu}$ means that, for $\nu \in \mathbb{R}^{*}$ we have a $\nu$ surgery on $\mathscr{E}$ analogous to surgery in equation (5.8). If $\nu=0$, the sub-index $\nu$ will generally be omitted.

In the complex analytic category our goal is twofold:

- to establish the analogous correspondence of (5.10),

- to examine the way that the "letters in an alphabet" (combinatorial pieces $W_{\iota}$ ) can fit together (in the metric category) to reconstruct a vector field $X$ on $(\mathbb{C}, 0)$. 
Definition 5.14. A singular analytic vector field germ representative $((\mathbb{C}, 0), X)$ determines a cyclic word at the singularity 0 , when in some punctured neighborhood of 0 , the germ admits a finite cyclic decomposition

$$
\mathcal{W}_{X}=W_{1} \cdots W_{k}, \quad W_{\iota} \quad \text { in the } C_{2 \pi / \lambda_{2}}, H, E_{\nu}, P_{\nu}, \mathscr{E}_{\nu} \text { alphabet }
$$

where the letters $W_{\iota}$ are read in a counterclockwise direction.

Example 5.15. (Motivational examples of how a vector field germ determines cyclic words).

1. Recalling the fifth column of Table 2, we see that zeros and poles give rise to natural cyclic words.

2. For slightly more complicated examples that include transcendental vector fields, first notice that the exponential vector field (Example 3.9) determines the cyclic word

$$
\left(\left(\widehat{\mathbb{C}}_{z}, \infty\right), X(z)=\mathrm{e}^{z} \frac{\partial}{\partial z}\right) \quad \longmapsto \quad \mathcal{W}_{X}=\mathscr{E} \mathscr{E}
$$

where we use the second representative for the letter $\mathscr{E}$ as in (5.5). Second, by considering the pullback via $z^{d}$, for $d \geq 2$, of $\mathrm{e}^{t} \frac{\partial}{\partial t}$, the new germ determines another cyclic word

$$
\left(\left(\widehat{\mathbb{C}}_{z}, \infty\right), X(z)=\frac{\mathrm{e}^{z^{d}}}{d z^{d-1}} \frac{\partial}{\partial z}\right) \longmapsto \mathcal{W}_{X}=\underbrace{\mathscr{E} \cdots \mathscr{E}}_{2 d} .
$$

3. In Example 8.32 consider Figure 15, upper drawing; the germ determines a cyclic word

$$
\left(\left(\widehat{\mathbb{C}}_{z}, \infty\right), X(z)=\mathrm{e}^{i \frac{\pi}{4}} \mathrm{e}^{z^{2}} \frac{\partial}{\partial z}\right) \quad \longmapsto \quad \mathcal{W}_{X}=H \mathscr{E} P_{\nu} \mathscr{E} H \mathscr{E} P_{-\nu} \mathscr{E}
$$

where clearly the residue of $\omega_{X}$ at $\infty \in \widehat{\mathbb{C}}_{z}$ is zero, hence the displacements $\nu$ associated to the parabolic sectors must cancel out. On the other hand, in Figure 16, upper drawing; the germ determines a cyclic word

$$
\left(\left(\widehat{\mathbb{C}}_{z}, \infty\right), X(z)=\mathrm{e}^{z^{2}} \frac{\partial}{\partial z}\right) \quad \longmapsto \quad \mathcal{W}_{X}=H \mathscr{E} \mathscr{E} H \mathscr{E} \mathscr{E} .
$$

It is to be noted that in this example the letters $H$ and $P_{\nu}$ fit together with the letter $\mathscr{E}$, forming nontrivial cyclic words. In this section we seek a better understanding of this phenomenon.

For other cyclic words arising from vector field germs see for instance Examples 11.5 and 11.6 in 11 .

Corollary 5.16. Let $((\mathbb{C}, 0), Y)$ be a singular analytic vector field germ. $Y$ determines a cyclic word $\mathcal{W}_{Y}$ at 0 as in fifth column of Table 2 if and only if $Y$ assumes the respective normal form.

Proof. Note that the letters $C_{2 \pi / \lambda_{2}}, H, E_{\nu}, P_{2 \pi i \lambda}$ in $\mathcal{W}_{Y}$ mean the (respective) angular sectors, in the flat surface category.

Given $Y$, we consider a representative of it on $D(0, \varepsilon)$ and a point $z_{0} \in D(0, \varepsilon)$. Thus,

$$
\Psi_{\text {uni }, Y}(z)=\int_{z_{0}}^{z} \omega_{Y}: D(0, \varepsilon) \backslash[0, \varepsilon] \longrightarrow \mathbb{C}_{t}
$$

is a univalued distinguished parameter of $(D(0, \varepsilon) \backslash[0, \varepsilon], Y)$. Moreover, $\Psi_{u n i, Y}$ is an isometry. 
The map $\Psi_{X}^{-1} \circ \Psi_{u n i, Y}: D(0, \varepsilon) \backslash[0, \varepsilon] \rightarrow(\mathbb{C}, 0)$ is well defined; see [69] pp. 139 for this order of ideas. Moreover, we can recognize an isometric glueing in the closure of its image (taking into account the displacement); whence by the above composition the map extends to a local isometry in the punctured disk $D(0, \varepsilon) \backslash\{0\}$. Using Remark 3.2 applied to $\Psi_{X}^{-1} \circ \Psi_{u n i, Y}$ the assertion follows.

It is clear that, if in $\mathcal{W}_{X}$ there appears $C_{2 \pi / \lambda_{2}}$, then necessarily $\mathcal{W}_{X}=C_{2 \pi / \lambda_{2}}$. Further note that, if in the $\mathcal{W}_{X}$ there appears $\mathscr{E}$, then the cyclic word will depend on the choice of germ representative, as will be explained in Remark 5.18 .

Remark 5.17. In all that follows, we recognize and use the equivalence between:

1. A letter $C_{2 \pi / \lambda_{2}}, H, E_{\nu}, P_{\nu}, \mathscr{E}_{\nu}$ in the alphabet.

2. The respective vector field germ $((\mathcal{A}, q), X)$; see Definition 5.2 .

3. The respective flat angular sector $\left((\mathcal{A}, q), g_{X}\right)$, provided with $\mathfrak{R e}(X)$.

5.9. Admissible words $\mathcal{W}$ at isolated essential singularities. Our departure point is an abstract cyclic word

$$
\mathcal{W}=W_{1} \cdots W_{k}, \quad W_{\iota} \text { in the } C_{2 \pi / \lambda_{2}}, H, E_{\nu}, P_{\nu}, \mathscr{E}_{\nu} \text { alphabet. }
$$

Notational conventions and clarifications.

1. A priori, an abstract cyclic word $\mathcal{W}$ does not arise from a vector field $X$, even though by construction each letter $W_{\iota}$ of the word does come from a vector field germ. In these cases we shall agree to not include $X$ as a sub-index of $\mathcal{W}$.

2. When in fact the cyclic word arises from a vector field we will include the sub-index $X$.

3. On the other hand when not needed we will omit the sub-indices in $C_{2 \pi / \lambda_{2}}$, $E_{\nu}, P_{\nu}, \mathscr{E}_{\nu}$

The following conditions are satisfied by $\mathcal{W}$.

- $W_{k+1}:=W_{1}$.

- Each $W_{\iota}=\left(\left(\mathcal{A}_{\iota}, q_{\iota}\right), X_{\iota}\right)$ can be interpreted also as a flat surface and an angular sector vector field germ, following Definition 5.7

- The geodesic boundary of $W_{\iota}$ coincides with the characteristic trajectories $\sigma_{\iota, 1}, \sigma_{\iota, 2}$ $\subset W_{\iota}$ as in $\$ 5.3$ and labels in the sub-index 1,2 are well determined by the counterclockwise orientation from the complex structure in $W_{\iota}$.

In order to perform the isometric glueing of the angular sectors of $\mathcal{W}$ in (5.12), we require the following additional rules (A)-(C), in accordance to Corollary [5.11] (A) There must be an even number (or zero) of angular sectors of each of the following types: hyperbolic, elliptic and entire sectors.

This follows from the fact that the characteristic trajectories of the angular sectors considered in the construction either arrive or escape the singularity $q_{\iota}$ of $W_{\iota}$, and when gluing them together the boundary orientations must match. The number of parabolic sectors can be arbitrary (including zero).

Rules (B) and (C) requires us to go back and examine the class 1 entire sector in more detail.

Remark 5.18 (A relation on words originating from choice of germ representative of class 1 entire sectors). In Figure 3 , the left and right strips

$$
\begin{aligned}
& \left\{z \mid 0 \leq \mathfrak{I m}(z) \leq \pi, \mathfrak{R e}(z) \leq \text { the respective } \mathfrak{R e}\left(\gamma_{1}\right)\right\} \cup\{\infty\}, \\
& \left\{z \mid 0 \leq \mathfrak{I m}(z) \leq \pi, \mathfrak{R e}(z) \geq \text { the respective } \mathfrak{R e}\left(\gamma_{2}\right)\right\} \cup\{\infty\}
\end{aligned}
$$


are such that $\mathrm{e}^{z} \frac{\partial}{\partial z}$ defines an elliptic $E$ and a hyperbolic sector $H$ on these. Using the language of letters and words, this geometric fact can be written as the abstract relations

$$
\mathscr{E} \sim E \mathscr{E} H \quad \text { or } \quad \mathscr{E} \sim H \mathscr{E} E,
$$

depending on the presence of $\mathscr{E}$ on upper or lower half-planes.

Furthermore, recalling from $\$ 5.3 .1$ the two different representatives for the class 1 entire sector $\mathscr{E}$, we observe that:

1) The half-logarithmic spiral (second representative) "should be" the definition for the class 1 entire sector $\mathscr{E}$. This is suggested by (5.13).

2) However, the half-logarithmic spiral is very nongeneric with respect to the choice of $\gamma=\gamma_{1} \cup \gamma_{2} \subset(\mathbb{C}, 0)$; see Figure 3. This follows by recalling that the border of the half-logarithmic spiral is a horizontal homoclinic trajectory of $X$ that starts and ends at the isolated essential singularity. So, assuming that $\gamma$ is an arc of circle of radius $r$ with centre the isolated essential singularity, then only a discrete set of radii $\left\{r_{\ell}\right\}$ will intersect the border of the half-logarithmic spiral (at the place where $\gamma$ bounces off). Thus any perturbation of the radius will give rise to a pair of sectors $E$ and $H$ as explained above.

Now, note that two characteristic trajectories $\sigma_{\iota, 2} \subset \partial W_{\iota}$ and $\sigma_{\iota+1,1} \subset \partial W_{\iota+1}$ can be glued together (see Corollary 5.11) if and only if both have finite or infinite $g_{X}$-length.

For example, an elliptic sector $E$ cannot be glued to a hyperbolic sector $H$. Since hyperbolic sectors have finite length boundaries while elliptic sectors have infinite length boundaries; the syllable $E H$ is forbidden.

In the context of Remark 5.18, this implies that there are two different types of class 1 entire sectors centred at $q$, depending on whether the left/right boundaries of the sector have finite length, or an infinite length. When needed we will denote these two types of class 1 entire sectors as

$$
{ }_{f} \mathscr{E}_{\infty} \text { and } \infty \mathscr{E}_{f}
$$

respectively (otherwise we will just denote class 1 entire sectors by $\mathscr{E}$ ); $\infty \mathscr{E}_{f}$ means that in the angular sector $\mathscr{E}$, the length of the characteristics trajectory $\sigma_{1}$ is infinite and the length of $\sigma_{2}$ is finite; see Figure 3 .

Thus, entire sectors $\mathscr{E}$ can be glued to entire, elliptic, hyperbolic or parabolic sectors.

(B) The syllables of angular sectors that can be constructed are

$$
\begin{gathered}
C, H H, E E, E P, P E, P_{\infty} \mathscr{E}_{f}, f_{f} \mathscr{E}_{\infty} P, E_{\infty} \mathscr{E}_{f}, f_{f} \mathscr{E}_{\infty} E, \\
H_{f} \mathscr{E}_{\infty}, \mathscr{E}_{f} H,{ }_{f} \mathscr{E}_{\infty} \mathscr{E}_{f}, \mathscr{E}_{f f} \mathscr{E}_{\infty} .
\end{gathered}
$$

(C) Following Remark 5.18, words admit the following replacement laws:

$$
\infty \mathscr{E}_{f} \sim E_{\infty} \mathscr{E}_{f} H, \quad{ }_{f} \mathscr{E}_{\infty} \sim H_{f} \mathscr{E}_{\infty} E .
$$

The first replacement means that, if we reduce the radius of an arc of circle $\gamma$ in 5.3 .1 used to bound class 1 entire sectors; we need to add sectors $E$ and $H$; to get $E_{\infty} \mathscr{E}_{f} H$. It is a reversible process for $X$ on a small enough disk $D(0, r)$. This reflects the auto-similar nature of class 1 entire sectors $\mathscr{E}$.

As a direct consequence of (C) the two abstract relations follow:

$$
P_{\infty} \mathscr{E}_{f} H \sim P E_{\infty} \mathscr{E}_{f} H H, \quad H_{f} \mathscr{E}_{\infty} P \sim H H_{f} \mathscr{E}_{\infty} E P .
$$


Definition 5.19. A cyclic word $\mathcal{W}$ is admissible if it obeys $(\mathrm{A})-(\mathrm{C})$.

We have therefore extended correspondence (5.10) to the complex analytic category

$$
((\mathbb{C}, 0), X) \longmapsto \mathcal{W}_{X}=W_{1} W_{2} \cdots W_{k}, \quad W_{\iota} \in\left\{C_{2 \pi / \lambda_{2}}, H, E_{\nu}, P_{\nu}, \mathscr{E}_{\nu}\right\}
$$

A natural question is to ask which germs $((\mathbb{C}, 0), X)$ give rise to admissible words. A first observation is that poles and zeros trivially determine admissible words; see Corollary 5.16. The complete answer to the above question and the recognition of analytic invariants arising from admissible words containing $\mathscr{E}$, will have to be postponed to 99, specifically Theorem 9.8. However, we can prove a natural result in this direction.

Proposition 5.20. Let $\mathcal{W}$ be an admissible word in the alphabet $C_{2 \pi / \lambda_{2}}, H, E_{\nu}$, $P_{\nu}$ and $\mathscr{E}_{\nu}$.

1) $\mathcal{W}$ comes from a germ $((\mathbb{C}, 0), X)$ of an isolated singularity of a complex analytic vector field.

2) Furthermore the sum of the displacements $\{\nu\}$ of $\mathcal{W}$ is $2 \pi i$ times the residue of $X$ at 0 .

3) If two germs $((\mathbb{C}, 0), X)$ and $((\mathbb{C}, 0), Y)$ give rise to the same admissible word, then they are holomorphically equivalent.

Proof. For (1), assume that the admissible word $\mathcal{W}$ has $k$ letters $\left\{W_{\iota}\right\}$. We glue, by isometries as in Corollary [5.11, the elliptic, hyperbolic, parabolic and entire angular sectors centred at $z=0$ following the anticlockwise cyclic order of the word $\mathcal{W}$. One needs to take into account the displacement $\nu$ of each letter $P_{\nu}, E_{\nu}$ and $\mathscr{E}_{\nu}$. In such a way, we obtain a Riemann surface, by abuse of notation $(\mathcal{W} \backslash\{0\}, J)$, it is homeomorphic to a topological annulus. Here $J$ is the conformal structure obtained from the flat metrics $W_{\iota}=\left(\mathcal{A}_{\iota} \backslash\left\{q_{\iota}\right\}, g_{X_{\iota}}\right)$ so, recalling the basic correspondence in Lemma 2.6. there is a unitary geodesic vector field on $(\mathcal{W} \backslash\{0\}, J)$.

We need to recognize that the ideal boundary 0 of $(\mathcal{W} \backslash\{0\}, J)$ (coming from the vertex points $\left.q_{\iota} \in W_{\iota}\right)$ is a conformal puncture of the conformal structure $J$.

Note that each angular sector $W_{\iota}$ determines in $(\mathcal{W} \backslash\{0\}, J)$ an angular sector of conformal type $\left\{z \in \mathbb{C}|0<| z \mid<\varepsilon_{\iota}, 0 \leq \arg (z) \leq \theta_{\iota}\right\}$. There exists a conformal map of type $\left\{z \mapsto z^{\beta}=w\right\}, \beta \in \mathbb{R}^{+}$, such that it sends the union of the angular sectors to one angular sector of argument $2 \pi$, i.e. to a conformal punctured disk. Thus, 0 is a conformal puncture of $J$.

Applying Lemma 2.6, to $\left(\mathcal{W} \backslash\{0\}, g_{X}, \mathfrak{R e}(X)\right)$, there is a germ $((\mathbb{C}, 0), X)$ of a singular analytic vector field that determines the admissible word $\mathcal{W}$ on $(\mathcal{W}, J)$.

For $(2)$, let $\mathcal{W}_{X}$ be an admissible word which comes from a germ $((\mathbb{C}, 0), X)$ of an isolated singularity having residue $\operatorname{Res}(X, 0)$. We define the residue of $\mathcal{W}_{X}$ as

$$
\operatorname{Res}\left(\mathcal{W}_{X}\right):=\operatorname{Res}(X, 0)
$$

For each syllable $W_{\iota} W_{\iota+1} \subset \mathcal{W}$, chosen from one of the following $E E, \mathcal{E} \mathcal{E}, \mathcal{E} E, \mathcal{E} P$, and the respective number $\nu \in \mathbb{C}^{*}$, we can perform a $\nu$-surgery that modifies the 
syllables as follows:

$$
\begin{aligned}
E E & \longmapsto\left\{\begin{array} { l l } 
{ E P _ { \nu } E } & { \nu \in \mathbb { C } \backslash \mathbb { R } , } \\
{ E _ { \nu } E } & { \nu \in \mathbb { R } , }
\end{array} \quad \mathcal { E } \mathcal { E } \longmapsto \left\{\begin{array}{ll}
\mathcal{E} P_{\nu} \mathcal{E} & \nu \in \mathbb{C} \backslash \mathbb{R}, \\
\mathcal{E}_{\nu} \mathcal{E} & \nu \in \mathbb{R},
\end{array}\right.\right. \\
\mathcal{E} E & \longmapsto\left\{\begin{array}{ll}
\mathcal{E} P_{\nu} E & \nu \in \mathbb{C} \backslash \mathbb{R}, \\
\mathcal{E}_{\nu} E & \nu \in \mathbb{R},
\end{array} \quad \mathcal{E} P_{\nu_{0}} \longmapsto \mathcal{E} P_{\nu_{0}+\nu} .\right.
\end{aligned}
$$

For the syllable $E E$, this was performed in case 5 of 98.5 . The other syllables admit the analogous surgery, it is required that the involved characteristic trajectories have infinite $g_{X}$-length in order to perform the isometric glueing. Using that the displacement is $2 \pi i$ times the residue (see (5.6) ), (2) follows.

For assertion (3), taking into account the displacements $\{\nu\}$ of $\mathcal{W}_{X}, \mathcal{W}_{Y}$, we observe that the flat metrics in $\left(\mathcal{W}_{X} \backslash\{0\}, g_{X}, \mathfrak{R e}(X)\right)$ and $\left(\mathcal{W}_{Y} \backslash\{0\}, g_{Y}, \mathfrak{R e}(Y)\right)$ are isometric. Using the ideas in the proof of Corollary [5.16 the result follows.

Example 5.21. 1. Displacements and residues in words $E \cdots E$ and $\mathscr{E} \cdots \mathscr{E}$, with $2 s$ letters. The word $E \cdots E$ with $-2 \pi \lambda_{2} \in \mathbb{R}$ surgery (see line five of Table 2), now can be written adding suitable parabolic sectors as

$$
P_{\nu_{1}} \underbrace{E \cdots E}_{2 s-3} P_{\nu_{2}} E \text {, satisfying }(\mathrm{A}-\mathrm{C}) \text { and } \nu_{1}+\nu_{2}=-2 \pi \lambda_{2} .
$$

The residue $\operatorname{Res}\left(\omega_{X}, 0\right)$ of the associated vector field germ $((\mathbb{C}, 0), X)$ of $P_{\nu_{1}} E \cdots E$ $P_{\nu_{2}} E$ at the singularity $z=0$ assumes the value $\left(\nu_{1}+\nu_{2}\right) / 2 \pi i \in \mathbb{C}^{*}$.

Similarly, the word $\mathscr{E} \mathscr{E}$, obtained from $\mathrm{e}^{z} \frac{\partial}{\partial z}$ at $\infty \in \widehat{\mathbb{C}}$ and having residue zero, gives origin to a family of new words $P_{\nu_{1}} \mathscr{E} P_{\nu_{2}} \mathscr{E}$, and new germs of vector fields, having residue $\left(\nu_{1}+\nu_{2}\right) / 2 \pi i \in \mathbb{C}^{*}$.

The analogous holds for $\mathscr{E} \cdots \mathscr{E}$, with $2 s \geq 4$ letters.

2. Let $X(z)=z \mathrm{e}^{z} \frac{\partial}{\partial z}$ be a singular analytic vector field on $\widehat{\mathbb{C}}$. Note that $X$ has singularities at 0 and $\infty$. The respective germs provide us with two words:

$$
H \mathcal{E} P_{2 \pi i} \mathcal{E} H \text { for }((\widehat{\mathbb{C}}, \infty), X), \quad P_{-2 \pi i} \text { for }((\widehat{\mathbb{C}}, 0), X) .
$$

\section{Poincaré-Hopf INDEX ThEORY}

Let us recall that the Poincaré-Hopf index $P H(X, 0) \in \mathbb{Z}$ of a singular analytic vector field germ $((\mathbb{C}, 0), X)$ having a zero at 0 , is the winding number of the Gauss map

$$
\mathfrak{G}: S_{\varepsilon}^{1} \longrightarrow S^{1}, \quad \gamma(\theta) \longmapsto \frac{\mathfrak{R e}(X(\gamma(\theta)))}{|\mathfrak{R e}(X(\gamma(\theta)))|},
$$

where $\gamma(\theta)=\varepsilon \mathrm{e}^{i \theta}:[0,2 \pi] \rightarrow(\mathbb{C}, 0)$ is an oriented circle having small enough radius $\varepsilon>0$ and $S^{1}$ is the unitary circle. Our starting point is the following.

Lemma 6.1. Let $\left((\mathbb{C}, 0), X(z)=f(z) \frac{\partial}{\partial z}\right)$ be singular analytic vector field germ having an isolated singularity at 0 .

1) The Poincaré-Hopf index, PH(X,0), as the winding number of the Gauss map is well defined.

2) As a consequence of the usual argument principle,

$$
P H(X, 0)=\frac{1}{2 \pi i} \int_{\gamma} \frac{f^{\prime}(z)}{f(z)} d z \quad \in \mathbb{Z} .
$$


Proof. The first assertion uses topological arguments. Let $\mathfrak{R e}(X)$ be the respective real analytic vector field (representative of the germ) on a punctured disk $D(0,2 \varepsilon) \backslash\{0\}=\{0<|z|<2 \varepsilon\}$.

We compute the winding number $\mathrm{w} \in \mathbb{Z}$ of the Gauss map of $\mathfrak{R e}(X)$, using a particular $\gamma(\theta)=\varepsilon \mathrm{e}^{i \theta}$.

Second, we note that the winding number w does not change under continuous deformations $\gamma(\theta, s):[0,2 \pi] \times[0,1] \rightarrow D(0,2 \varepsilon) \backslash\{0\}$ of $\gamma(\theta):=\gamma(\theta, 0)$, as an element in the fundamental group of the punctured disk; as long as each path $\gamma\left(\theta, s_{0}\right)$ does not go through any singularities of $\mathfrak{R e}(X)$. In the punctured disk, the vector field $\mathfrak{R e}(X) /|\mathfrak{R e}(X)|$ is $C^{0}$ (in fact real analytic) and $\gamma(\theta, s)$ is a continuous deformation.

Example 6.2. 1. If $X$ is meromorphic at 0, then

$$
P H(X, 0)=\left\{\begin{array}{cl}
-k \leq-1 & \text { for } 0 \text { a pole of multiplicity }-k, \\
0 & \text { for } 0 \text { a regular point } \\
s \geq 1 & \text { for } 0 \text { a zero of multiplicity } s
\end{array}\right.
$$

2. Moreover, let $X$ be a meromorphic vector field on a compact Riemann surface $M$ of genus $\mathfrak{g}$. Then

$$
\sum_{q \in M} P H(X, q)=2-2 \mathfrak{g}=c_{1}\left(T^{\prime} M\right)
$$

here, the last equality is the Chern class $c_{1}$ of the holomorphic tangent line bundle $T^{\prime} M$.

In the case of poles, (6.3) is proved as follows. There exists $\rho:(\mathbb{C}, 0) \rightarrow \mathbb{R}^{+} \cup\{0\}$ a function germ, such that $\rho \mathfrak{R e}(X)$ is a $C^{1}$ vector field on $(\mathbb{C}, 0)$, hence $P H(X, 0):=$ $P H(\rho \mathfrak{R e}(X), 0)$ is well defined.

In fact, for $X(z)=\frac{1}{z^{k}} \frac{\partial}{\partial z}$, the required function is $\rho(z)=|z|^{2 k}$.

Using Table 2 and the $C^{1}$ Poincaré-Hopf index theory (see for example W. Thurston's exposition 69, $\$ 1.3)$, the proof of $(6.3)$ is complete.

Let $((\mathbb{C}, 0), X)$ be a singular analytic vector field germ having an isolated singularity at 0 determining an admissible word $\mathcal{W}_{X}$, let us denote: $h, e, \epsilon$ the number of hyperbolic, elliptic or class 1 entire sectors in $\mathcal{W}_{X}$, respectively.

Remark 6.3. Note that since $h, e, \epsilon$ are obtained from the cyclic word $\mathcal{W}_{X}$, these numbers are in fact associated to a germ representative. Thus even though they depend individually on the germ representative, the sum $e-h+\epsilon$ does not, as a consequence of Rule (C) in $\$ 5.9$.

The assertion Theorem (A.1) below, shows that one can in fact calculate the Poincaré-Hopf index of a vector field at a singularity 0 by knowing the type of sectors centred at 0 that occur. It generalizes the classical formula of I. Bendixon; see [6] appendix $\S 10$.

Theorem (A) (Local and global Poincaré-Hopf theory). 1) Let ((C, 0), X) be a germ of singular analytic vector field with an isolated singularity at 0 and further suppose that $X$ determines an admissible word $\mathcal{W}_{X}$. Then the Poincaré-Hopf index of $X$ at 0 is

$$
P H(X, 0)=1+\frac{e-h+\epsilon}{2} .
$$


2) Let $X$ be a singular analytic vector field on a compact $M$ having a discrete set of poles, zeros and essential singularities determining admissible words at each singularity, then

$$
\chi(M)=\sum_{q \in M} P H(X, q) .
$$

In order to compute the winding number of $(X, 0)$, our main tool is the argument principle on angular sectors.

Let $X(z)=f(z) \frac{\partial}{\partial z}$ be a nonvanishing holomorphic vector field on an angular sector $\left\{z|0<| z \mid<2 \varepsilon, 0 \leq \arg (z) \leq \theta_{0}\right\}$ (note that $z=0$ is removed) such that the two boundary rays $\left\{\arg (z)=0, \theta_{0}\right\}$ are trajectories of $\pm \mathfrak{R e}(X)$, and consider the path $\gamma(\theta)=\varepsilon \mathrm{e}^{i \theta}:\left[0, \theta_{0}\right] \rightarrow \mathbb{C}$ in the sector.

The winding argument of $X$ in the angular sector is

$$
\mathfrak{G}\left(\gamma\left(\theta_{0}\right)\right)-\mathfrak{G}(\gamma(0))=\frac{1}{i} \int_{\gamma(0)}^{\gamma\left(\theta_{0}\right)} \frac{f^{\prime}(z)}{f(z)} d z \quad \in \mathbb{R} .
$$

The result must be interpreted using the endpoints of the trajectory $\mathfrak{G} \circ \gamma$ in $S^{1}$, in particular, this winding number can be greater than $2 \pi$ or less than $-2 \pi$.

Two important facts:

i) The boundary values of $\mathfrak{G}$ are independent of the points in the corresponding boundary rays, more precisely,

$$
\begin{gathered}
\mathfrak{G}(\rho \gamma(0))=\mathfrak{G}(\gamma(0))= \pm 1 \in S^{1}, \\
\mathfrak{G}\left(\rho \gamma\left(\theta_{0}\right)\right)=\mathfrak{G}\left(\gamma\left(\theta_{0}\right)\right)
\end{gathered}
$$

the signs \pm depend on whether $\mathfrak{R e}(X)$ points to 0 or not, along the boundary ray $\{\arg (z)=0\}$. Whence, the integral (6.7) is independent of every simple path, having extreme points in the boundary rays, required to compute it (using an argument analogous to the one in Lemma 6.1,1).

ii) The integral (6.7) does not require that $X$ assume a continuous value at the vertex $z=0$ of the angular sector.

Proof of Theorem (A.1). The case $W=C$ is obvious. If the letters $W \in\{H, E, P, \mathscr{E}\}$ describe the kind of sectors in $\mathcal{W}_{X}$ and letting $j$ run through the number of sectors of each kind (for instance $j \in\{1, \ldots, h\}$ in the case of the hyperbolic sectors; $e, p, \epsilon$ respectively for $E, P$, and $\mathscr{E})$, then we decompose the path $\gamma(\theta)=\varepsilon \mathrm{e}^{i \theta}:[0,2 \pi] \rightarrow$ $(\mathbb{C}, 0)$ in closed connected arcs of argument $\left\{\theta_{L, j}\right\}$ coming from the decomposition of $\mathcal{W}_{X}$ in angular sectors. Thus, by construction

$$
\sum_{j=1}^{h} \theta_{H, j}+\sum_{j=1}^{e} \theta_{E, j}+\sum_{j=1}^{p} \theta_{P, j}+\sum_{j=1}^{\epsilon} \theta_{\mathscr{E}, j}=2 \pi
$$

are the (unordered) arguments of the angular sectors. In order to simplify the notation, we apply suitable coordinate changes on each angular sector in $(\mathbb{C}, 0)$, and recognize $\gamma\left(\left[0, \theta_{L, j}\right]\right)$ as the arc of $\gamma$ in an angular sector having a boundary in $\mathbb{R}^{+}$, similar to what was done for the sector in (6.7) and (6.8).

Lemma 6.4. In each angular sector determined by $\gamma\left(\left[0, \theta_{L, j}\right]\right)$, the winding argument of its Gauss map $\mathfrak{G} \circ \gamma$ behaves as follows.

1) In a hyperbolic sector it is $\theta_{H, j}-\pi$.

2) In an elliptic sector it is $\theta_{E, j}+\pi$. 
3) In a parabolic sector it is $\theta_{P, j}$.

4) In an entire 1 sector it is $\theta_{\mathscr{E}, j}+\pi$.

Following in some places the original ideas of I. Bendixon and $C^{1}$ arguments a proof of Lemma 6.4 $1-3$, is given in [6], appendix $\S 10$. We use the argument principle 6.7).

Proof of Lemma 6.4.1.

Step 1. Computation in a particular hyperbolic sector.

We assume that $\theta_{H, j}=2 \pi /(2 k+2)$, for $-k \leq-1$. Let $X(z)=\frac{1}{z^{k}} \frac{\partial}{\partial z}$ be the vector field on the sector $\left\{z \mid 0 \leq \arg (z) \leq \theta_{H, j}\right\}$, it follows that

$$
\frac{1}{i} \int_{\gamma(0)}^{\gamma\left(\theta_{H, j}\right)} \frac{f^{\prime}(z)}{f(z)} d z=\frac{1}{i} \int_{\gamma(0)}^{\gamma\left(\theta_{H, j}\right)} \frac{-k}{z} d z=\frac{-k 2 \pi}{2 k+2}=\frac{2 \pi}{2 k+2}-\pi .
$$

Step 2. Computation in any hyperbolic sector.

a)

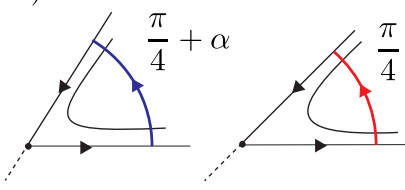

b)

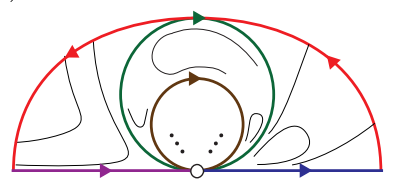

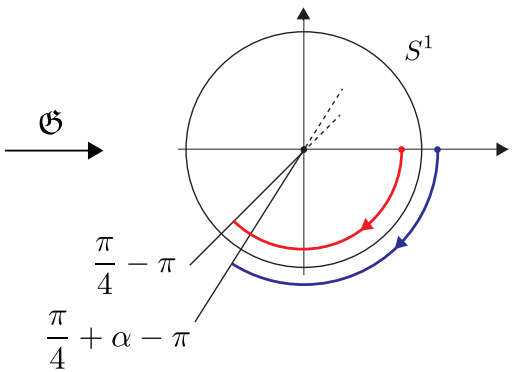
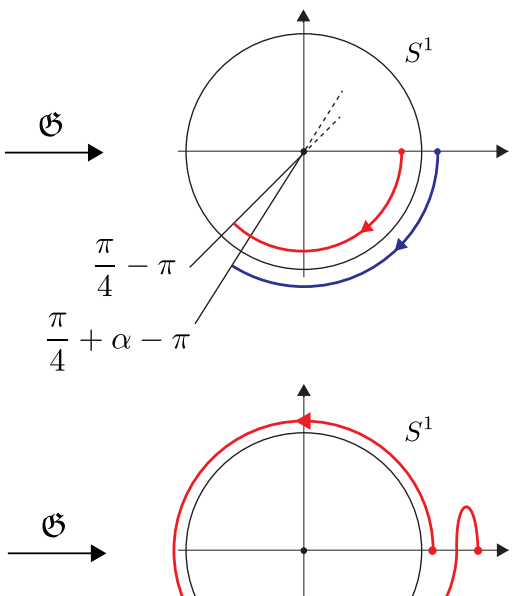

Figure 8. a) The winding argument of a hyperbolic sector $H$ of angle $\theta_{H, j}$ is $\theta_{H, j}-\pi$. b) The winding argument of a entire sector $\mathscr{E}$ of angle $\theta_{\mathscr{E}, j}:=\pi$ is $\pi+\theta_{\mathscr{E}, j}$. Furthermore, both results persist under small perturbations of $\alpha$. See proof of Lemma 6.4

We assume $\left\{\theta_{H, j}<2 \pi\right\}$, i.e. not necessarily of the form $2 \pi /(2 k+2)$.

Consider the map $\left\{w \mapsto w^{\beta}=z\right\}$, for suitable $\beta \in(0,2 \pi)$, sending the angular sector $\left\{0 \leq w \leq \theta_{H, j}\right\}$ to a sector as the one that appears in (6.10), and compute the respective integral of type (6.7).

A more geometric argument is as follows. Assume (6.10) and consider a small enough perturbation of the angle $\theta_{H, j}+\alpha$ say, for example, $|\alpha|<2 \pi /(2 k+2)$.

Let $\mathfrak{G}$ be the Gauss map of the original vector field (6.10), sending the counterclockwise oriented arc $\gamma\left(\left[0, \frac{2 \pi}{2 k+2}\right]\right) \subset S_{\varepsilon}^{1}$ to the clockwise oriented arc in $S^{1}$ having extremes (similarly as in equation (6.8)): 
$\mathfrak{G}(\gamma(0))=1$, i.e. the ray $\{\arg (z)=0\}$ is a trajectory of $\mathfrak{R e}(X)$ that does not point to 0 ;

$\mathfrak{G}\left(\gamma\left(\frac{2 \pi}{2 k+2}\right)\right)=\frac{2 \pi}{2 k+2}-\pi$.

See Figure 8 a. for $k=3$.

Now, we increase the original argument by $\alpha$; translate the vector field $X$ to the new angular sector of angle $(2 \pi /(2 k+2))+\alpha$, obtaining a new vector field $X_{\alpha}$ (this can be done with a map of type $\left.\left\{w \mapsto w^{\beta}=z\right\}\right)$.

The Gauss map of the vector field $X_{\alpha}$, sends the arc $\gamma\left(\left[0, \frac{2 \pi}{2 k+2}+\alpha\right]\right) \subset S_{\varepsilon}^{1}$ to the clockwise oriented arc in $S^{1}$ having extremes, similarly as in equation (6.8):

$\mathfrak{G}(\gamma(0))=1$;

$\mathfrak{G}\left(\gamma\left(\frac{2 \pi}{2 k+2}+\alpha\right)\right)=\frac{2 \pi}{2 k+2}+\alpha-\pi$, i.e. the ray $\left\{\arg (z)=\frac{2 \pi}{2 k+2}+\alpha\right\} \subset \mathbb{C}^{*}$ is a trajectory of $\mathfrak{R e}\left(X_{\alpha}\right)$ that points to 0 .

See Figure 8 a. The proof of Lemma 6.4.1 is done.

Example 6.5 (Coherence with previous results). 1. If $\theta_{H, j}=\pi$, then we can use $X(z)=\frac{\partial}{\partial z}$ and

$$
\frac{1}{i} \int_{\gamma(0)}^{\gamma(\pi)} \frac{f^{\prime}(z)}{f(z)} d z=0=\theta_{H, j}-\pi .
$$

2. An alternative proof of (6.3) follows (see [31, 52]), since the total winding argument of a pole of order $-k$ is

$$
\frac{1}{i} \int_{\gamma(0)}^{\gamma(2 \pi)} \frac{-k}{z} d z=-k 2 \pi .
$$

The proof of Lemma 6.4, $2-3$ follows the same ideas. If the particular elliptic sector describes argument $\theta_{E, j}=\pi$, then we can use $\left((\mathbb{C}, 0), z^{2} \frac{\partial}{\partial z}\right)$ and

$$
\frac{1}{i} \int_{\gamma(0)}^{\gamma(\pi)} \frac{f^{\prime}(z)}{f(z)} d z=\frac{1}{i} \int_{\gamma(0)}^{\gamma(\pi)} \frac{2}{z} d z=2 \pi=\theta_{E, j}+\pi .
$$

Proof of Lemma 6.4.4.

Step 1. Computation in a particular class 1 entire sector.

We assume that $\theta_{\mathscr{E}, j}=\pi$. Let $X(z)=-z^{2} \mathrm{e}^{1 / z} \frac{\partial}{\partial z}$ be the vector field on $\{z \mid 0 \leq$ $\arg (z) \leq \pi\} \backslash\{0\}$, we get

$$
\frac{1}{i} \int_{\gamma(0)}^{\gamma(\pi)} \frac{f^{\prime}(z)}{f(z)} d z=\frac{1}{i} \int_{\gamma(0)}^{\gamma(\pi)}\left(\frac{2}{z}+\frac{1}{z^{2}}\right) d z=2 \pi=\theta_{\mathscr{E}, j}+\pi .
$$

Note that, the Gauss map has a critical point at some $\gamma\left(\theta_{0}\right) \in S_{\varepsilon}^{1}$ in the second quadrant; see Figure 8,b.

Step 2. The computation in any class 1 entire sector describing an argument $\pi+\alpha$, is analogous as in the case of hyperbolic sectors.

The proof of Lemma 6.4.4 is done.

Finally, by Lemma 6.4 the winding number of $((\mathbb{C}, 0), X)$ is

$$
\frac{1}{2 \pi} \mathfrak{G} \circ \gamma\left(\coprod_{j=1}^{h} \theta_{H, j} \coprod_{j=1}^{e} \theta_{E, j} \coprod_{j=1}^{p} \theta_{P, j} \coprod_{j=1}^{\epsilon} \theta_{\mathscr{E}, j}\right)=-\frac{h}{2}+\frac{e}{2}+1+\frac{\epsilon}{2},
$$


where by abuse of notation, the union means the decomposition of $\gamma:[0,2 \pi] \rightarrow$ $(\mathbb{C}, 0)$ in intervals from the angular sectors of $X$, as seen in (6.9).

The proof of Theorem (A.1) is done.

For the second part in Theorem (A), it will be suitable to remember a deep result of C. Gutierrez [30] and to do a digression on the differentiability required to develop Poincaré-Hopf theory.

Theorem 6.6 (C. Gutierrez's smoothing of singular continuous flows and foliations). Let $\mathcal{M}$ be a $C^{\infty}$ compact, orientable two manifold without boundary.

1) Let $\varphi: \mathbb{R} \times \mathcal{M} \rightarrow \mathcal{M}$ be a continuous flow. Then there exists a $C^{1}$ flow on $\mathcal{M}$ which is topologically equivalent to $\varphi$. Furthermore, if all minimal sets of $\varphi$ are trivial, then $\varphi$ is topologically equivalent to a $C^{\infty}$ flow.

2) Let $\mathcal{F}$ be a continuous one dimensional orientable foliation with singularities on $\mathcal{M}$. If the set of singularities of $\mathcal{F}$ is compact, then there exists a $C^{1}$ flow on $\mathcal{M}$ which is topologically equivalent with $\varphi$.

Thus, for a continuous one dimensional orientable foliation with singularities, $\mathcal{F}$ on $\mathcal{M}$ as in Theorem 6.6, 2, the Poincaré-Hopf formula

$$
\chi(\mathcal{M})=\sum_{q \in \mathcal{M}} P H(\mathcal{F}, q)
$$

holds, particularly if $P H(\mathcal{F}, q)$ is well defined.

If in addition $\mathcal{F}$ does not have exceptional minimal sets in $\mathcal{M}$, then $\varphi$ can be smoothed to a $C^{\infty}$ flow.

Proof of Theorem (A.2). We look at the singular analytic vector field $X$ on $M$ on a disk centred at a singularity, as usual we denote it by $((D(0, \varepsilon), 0), X)$.

Let $\rho: D(0, \varepsilon) \rightarrow \mathbb{R}^{+} \cup\{0\}$ be a suitable $C^{\infty}$ bump function germ vanishing at infinite order at the origin 0 , i.e. $\rho(\overline{0})=0$ exactly at the origin 0 and whose Taylor series is identically zero.

Note that $\rho \mathfrak{R e}(X) /|\mathfrak{R e}(X)|$ is a $C^{\infty}$ real vector field on a punctured vicinity $D(0, \varepsilon) \backslash\{0\}$ and it is a $C^{0}$ vector field on a whole disk $D(0, \varepsilon)$, with an isolated zero at 0 .

Let $g$ be any $C^{\infty}$ Riemannian metric on the compact Riemann surface $M$. Using partitions of the unity and the above trick; there exists a global $C^{\infty}$ function $\rho_{1}: M \rightarrow \mathbb{R}^{+} \cup\{\infty\}$ such that $X_{1}:=\rho_{1} \mathfrak{R e}(X) /|\mathfrak{R e}(X)|_{g}$ is $C^{0}$ on $M$ and $C^{\infty}$ on $M \backslash \mathcal{S}_{X}$

We can apply Gutierrez's Theorem 6.6 to the foliation $\mathcal{F}$ defined by $X_{1}$ on $M$. There exists an associated $C^{1}$ flow and one $C^{1}$ vector field, say $X_{2}$. Now, we apply the usual $C^{1}$ Poincaré-Hopf index theory.

The proof of Theorem (A.2) is done.

Example 6.7 (Index for admissible words of singular analytic vector fields on $\widehat{\mathbb{C}}$ ).

1. The vector field $X(z)=\mathrm{e}^{z} \frac{\partial}{\partial z}$ has associated the word

$\mathscr{E} \mathscr{E}$.

2. Let $X(z)=\mathrm{e}^{z^{2}} \frac{\partial}{\partial z}$ be an entire vector field, its unique singularity is at $\infty$ having associated word (see Figure 16) 
Comparing with Example 5.15,2, we see that the cyclic word representative is not unique, as is explained in Remark 5.18.

3. On the other hand, let $X(z)=\left(d z^{d-1}\right)^{-1} \mathrm{e}^{z^{d}} \frac{\partial}{\partial z}=\left(z^{d}\right)^{*}\left(\mathrm{e}^{t} \frac{\partial}{\partial t}\right)$, for $d \geq 2$, be a singular analytic vector field with a pole at $z=0$ and an isolated essential singularity at $\infty$. So by using that $X$ is a pullback, as in $\$ 3$,

$$
\underbrace{H \ldots H}_{2 d}, \quad \underbrace{\mathscr{E} \ldots \mathscr{E}}_{2 d}
$$

are the associated words, respectively.

In the three cases,

$$
\sum_{q \in \widehat{\mathbb{C}}} P H(X, q)=2 .
$$

4. Furthermore, the vector field $X(z)=\mathrm{e}^{z} \mathrm{e}^{-\mathrm{e}^{z}} \frac{\partial}{\partial z}$ has a unique singularity at $\infty$, having associated word (see Figures 2, 5)

$$
\mathscr{E} \mathscr{E} \mathscr{E} \mathscr{E} \text {. }
$$

Whence, using Theorem 6.6 and the above ideas, it is possible to show that in this case, once again $P H(X, \infty)=2$.

Remark 6.8. Notice that rule (A) of the construction of admissible words has an equivalent statement in terms of the Poncaré-Hopf index:

$\left(\mathrm{A}^{\prime}\right)$ A vector field $X$ arising from an admissible word $\mathcal{W}$ must have Poincaré-Hopf index an integer.

We define the Poincaré-Hopf index of an admissible word $P H\left(\mathcal{W}_{X}\right)$ using (6.5). Of course $P H\left(\mathcal{W}_{X}\right)=P H(X, 0)$.

\section{Extending Vector Field germs to compact Riemann surfaces}

In this section we shall explore two results on the extension of germs. The first result is more general in the sense that it does not require that the singularity be isolated, as is required in the second.

Theorem 7.1 (Extension to some compact Riemann surface). Let ((C, 0$), X)$ be a germ of a singular analytic vector field having a nonnecessarily isolated singularity at 0 . There exists an extended singular analytic vector field $\widetilde{X}$ on a compact Riemann surface $M_{\mathfrak{g}}$, for each genus $\mathfrak{g} \geq 0$, such that the germ of $\widetilde{X}$ at some $p \in M_{\mathfrak{g}}$ is holomorphically equivalent to the germ $X$, with $\tilde{X}$ having an additional finite number of zeros and poles.

Proof. Let $\gamma:[0,1] \rightarrow(\mathbb{C}, 0)$ be a simple closed path enclosing 0 , without loss of generality we assume that $\gamma$ avoids the singularities of a germ representative of $X$ on $(\mathbb{C} \backslash\{0\}, 0)$.

Let $\Gamma=\Psi \circ \gamma \subset \mathbb{C}_{t}$ be the image path under the distinguished parameter. $\Gamma$ is closed if and only if $\int_{\gamma} \omega_{X}$ is zero.

By a small enough perturbation of $\gamma$ we can assume the following conditions.

1) $\Gamma$ is a polygonal (i.e. piecewise linear) path having edges $\Gamma_{\iota}$ and vertices $v_{\iota}$,

$$
\Gamma=\left\{v_{0}\right\} \cup \Gamma_{1} \cup\left\{v_{1}\right\} \cup \ldots \Gamma_{s} \cup\left\{v_{s}\right\} \subset \mathbb{C}_{t},
$$

with exactly $s+1$ different vertices if and only if $\int_{\gamma} \omega_{X} \neq 0$, or exactly $s$ different vertices (in which case $v_{0}=v_{s}$ ) if $\int_{\gamma} \omega_{X}=0$. 
2) If $\iota \neq j$, then $\Gamma_{\iota} \cap \Gamma_{j}$ is empty or at most one point.

3) A half $\epsilon$-tubular neighborhood $\mathcal{T} \subset \mathbb{C}_{t}$ of $\Gamma$ is well defined, for $\varepsilon>0$ small enough, such that $\Psi^{-1}(\mathcal{T})$ is a topological annulus inside $\gamma \subset(\mathbb{C}, 0)$, homeomorphic to $S^{1} \times(-\varepsilon, 0]$.

Let $\sigma$ be a piece of the trajectory of $\mathfrak{R e}(X)$ in $(\mathbb{C}, 0)$ having initial condition $\gamma(0)=v_{0}$. $\mathcal{T}$.

If the residue is zero, then $\Sigma=\Psi(\sigma) \subset \mathbb{C}_{t}$ is exactly a horizontal trajectory in

If the residue is nonzero, then there are $\Sigma_{1}=\Psi(\sigma), \Sigma_{2}=\Psi(\sigma) \subset \mathbb{C}_{t}$ two horizontal trajectories in its image (by considering for $\Sigma_{2}$, the analytic extension of $\Psi$ along $\gamma$ ). Moreover, $\Sigma_{1}, \Sigma_{2}$ are in the boundary of $\mathcal{T}$; see central drawings in Figure 9 a-b.

As first stage we want to recover $M_{\mathfrak{g}}=\widehat{\mathbb{C}}$.

Case 1. $\Gamma \subset \mathbb{C}_{t}$ does not have self-intersections and is closed.

Assume that $\mathcal{T}$ is outside of $\Gamma$. The obvious solution is by gluing the closed polygonal region $\mathcal{Q} \subset\left(\mathbb{C}, \frac{\partial}{\partial t}\right)$ which is bounded by $\Gamma$, using Corollary [5.11, No additional singularities of $\widetilde{X}$ appear. In this case, the original germ necessarily satisfies $P H(X, 0)=2$.

On the other hand, assume that $\mathcal{T}$ is inside of $\Gamma$, i.e. $\mathcal{T}$ determines the interior half $\varepsilon$-tubular neighborhood of a closed polygonal region $\mathcal{Q} \subset\left(\mathbb{C}, \frac{\partial}{\partial t}\right)$. The obvious solution is by glueing the closed complement $\overline{\mathcal{Q}^{c}} \subset\left(\widehat{\mathbb{C}}, \frac{\partial}{\partial t}\right)$ in the Riemann sphere. An additional double zero of $\widetilde{X}$ appears. In this case, the original germ necessarily satisfies $P H(X, 0)=0$.

Moreover, a second solution will be illustrative in the other cases.

Example 7.2. Let $((\mathbb{C}, 0), X)$ be a germ as in the case 1 above, and assume that $\mathcal{T}$ determines the interior half $\varepsilon$-tubular neighborhood of a closed polygonal region $\mathcal{K} \subset \mathbb{C}_{z}$ having $s \geq 3$ edges $\left\{\Gamma_{\iota}\right\}$.

$\Gamma$ is a polygonal, having $s \geq 3$ edges $\left\{\Gamma_{\iota}\right\}$ (as vectors in $\mathbb{C}$ using the counterclockwise orientation in the boundary $\partial \mathcal{T}$ ). The sum of the internal angles $\left\{\theta_{\iota} \mid \iota=1, \ldots, s\right\}$ of $\mathcal{T}$ is $(s-2) \pi$. We identify all the vertices of $\mathcal{T}$ to the same point $q$; obtaining a topological sphere $S^{2}$ minus a bouquet of $s$ open disks. Applying Corollary [5.11, we glue to each closed geodesic $\Gamma_{\iota} \cup\{q\}$ a semi-infinite height cylinder $S_{\left|\Gamma_{\iota}\right|}^{1} \times[0, \infty)$, where the sub-index $\left|\Gamma_{\iota}\right|$ denotes the perimeter.

We can recognize that every cylinder contributes with cone angle $\pi$ around $q$ and the point $q$ has a cone angle $(2 s-2) \pi$. Hence $q$ is a pole of order $2-s$ of a new vector field $\tilde{X}$. The riemannian end of each cylinder is a simple zero of $\widetilde{X}$, recalling that each cylinder comes from $\left(\overline{D(0,1)}, \pm i\left(2 \pi /\left|\Gamma_{\iota}\right|\right) z \frac{\partial}{\partial z}\right)$, as in Table 2 .

By the uniformization theorem, the resulting Riemann surface is $\widehat{\mathbb{C}}$. The resulting vector field $\widetilde{X}$ on $\widehat{\mathbb{C}}$ has the original singularity germ $X$ at some $p \in \widehat{\mathbb{C}}, s$ simple zeros, and one pole at $q$ of order $-(s-2)$.

(The above generalizes the following: if $X$ is nonsingular in the interior of $\mathcal{T}$, then $\tilde{X}$ is a polynomial vector field $P(z) \frac{\partial}{\partial z}$ on $\widehat{\mathbb{C}}_{z}$ with $s$ simple zeros having residues $\Gamma_{\iota} / 2 \pi i$. See Figures 2-4 in [50] and Figure 1 in [49].)

Case 2. $\Gamma \subset \mathbb{C}_{t}$ satisfies $s=2$ in (17.1), self-intersections are possible. 
We consider two sub-cases depending on the position of the half $\varepsilon$-tubular neighborhood $\mathcal{T}$ with respect to $\Gamma=\left\{v_{0}\right\} \cup \Gamma_{1} \cup\left\{v_{1}\right\} \cup \Gamma_{2} \cup\left\{v_{2}\right\}$; see Figure9a- $\mathrm{a}$. Without loss of generality, we assume $\mathfrak{I m}\left(v_{0}\right)<\mathfrak{I m}\left(v_{2}\right)$ and $\mathfrak{R e}\left(v_{0}\right)<\mathfrak{R e}\left(v_{1}\right) . \Psi(\sigma)$ has two associated horizontal trajectories $\Sigma_{1}, \Sigma_{2}$ in $\mathbb{C}_{t}$, arriving at $v_{0}$ and $v_{2}$.

- In Figure 9, a we identify the vertices $v_{0}, v_{1}, v_{2}$ to a point $q$. The cone angle of $q$, determined by $\mathcal{T}$, is

$$
\angle\left(\Gamma_{1},-\mathfrak{R e}\left(\frac{\partial}{\partial t}\right)\right)+\angle\left(\Gamma_{2},-\Gamma_{1}\right)+\angle\left(-\mathfrak{R e}\left(\frac{\partial}{\partial t}\right),-\Gamma_{2}\right)=4 \pi .
$$

We glue to each geodesic $\Gamma_{j} \cup\{q\}$ a semi-infinite height cylinder $S_{\left|\Gamma_{j}\right|}^{1} \times[0, \infty)$. The point $q$ has a total cone angle $6 \pi$, hence it determines a pole of order -2 ; see Table 2. The riemannian end of each cylinder gives origin to a simple zero in $\tilde{X}$; see Table 2, $\widetilde{X}$ on $\widehat{\mathbb{C}}$ has additionally a double pole and two simple zeros.

Let us make two remarks.

i) Equation (7.2) is independent of the assumptions $\mathfrak{I m}\left(v_{0}\right)<\mathfrak{I m}\left(v_{2}\right)$ and $\mathfrak{R e}\left(v_{0}\right)<\mathfrak{R e}\left(v_{1}\right)$. The angle $4 \pi$ remains constant under continuous deformation of $\Gamma$.

ii) The choice of the cone angle determined by $\mathcal{T}$ at the extreme vertices $v_{0}$ and $v_{s}$ in the drawings (a)-(f), is due to the fact that the glueing of $\mathcal{T}$ must be an oriented annulus (we must glue the geodesics of the vectors $\mathfrak{R e}\left(\frac{\partial}{\partial t}\right)$ at $v_{\iota}$, as in Corollary 5.11).

- In Figure 9, b we identify the vertices $v_{0}, v_{1}, v_{2}$ to a point $q$. The cone angle of $q$ determined by $\mathcal{T}$ is $2 \pi$. We glue to each geodesic $\Gamma_{j} \cup\{q\}$ a semi-infinite height cylinder. The point $q$ has a total cone angle $4 \pi$ and gives origin to a pole of order -1 of $\tilde{X}$; see Table 2. The riemannian end of each cylinder is a simple zero, see Table 2 Hence $\widetilde{X}$ on $\widehat{\mathbb{C}}$ has additionally a simple pole and two simple zeros.

Case 3. $\Gamma \subset \mathbb{C}_{t}$ satisfies $s=3$ in (7.1), self-intersections are possible.

We consider four sub-cases depending on the position of $\mathcal{T}$ respect to $\Gamma=$ $\left\{v_{0}\right\} \cup \Gamma_{1} \cup\left\{v_{1}\right\} \cup \Gamma_{2} \cup\left\{v_{2}\right\} \cup \Gamma_{3} \cup\left\{v_{3}\right\}$ and the possible self-intersections of $\Gamma$; see Figure 9, $\mathrm{c}-\mathrm{f}$. We assume without loss of generality that $\mathfrak{I m}\left(v_{0}\right)<\mathfrak{I m}\left(v_{2}\right)$ and $\mathfrak{R e}\left(v_{0}\right)<\mathfrak{R e}\left(v_{1}\right)$. The two horizontal edges at the left of each figure are the images of the trajectories of $\mathfrak{R e}\left(\frac{\partial}{\partial t}\right)$ at $v_{0}$ and $v_{3}$.

- Considering Figure 9c we identify the four vertices to the same point $q$. The cone angle at $q$ determined by $\mathcal{T}$ is $5 \pi$. We glue to each circle $\Gamma_{j} \cup\{q\}$ a semi-infinite height cylinder. The point $q$ has a total cone angle of $8 \pi$ and is a pole of order -3 of $\tilde{X}$. The Riemannian end of each cylinder is a simple zero of $\widetilde{X}$. Thus the resulting riemann surface is $\widehat{\mathbb{C}}$.

The computations for drawings (d), (e), (f) are similar, we indicate in Figure 9 the cone angle determined by $\mathcal{T}$ at the new singularity $q$.

Case 4 . $\Gamma \subset \mathbb{C}_{t}$ satisfies $s \geq 4$ in (7.1), self-intersections are possible.

We use an inductive construction process with the above ideas. We leave for the reader the details of the computation of the cone angle for any $s \geq 4$.

The case of $\widetilde{X}$ on $M_{\mathfrak{g}}=\widehat{\mathbb{C}}$ is done.

The second stage is $M_{\mathfrak{g}}$, for $\mathfrak{g} \geq 1$.

We perform the isometric connected sum of the flat surfaces $\left(\widehat{\mathbb{C}}, \widetilde{X_{1}}\right)$ and suitable compact Riemann surface $\left(N, X_{2}\right)$; cutting $\mathbb{C}$ along one segment $\Sigma$ of trajectory of 
a)

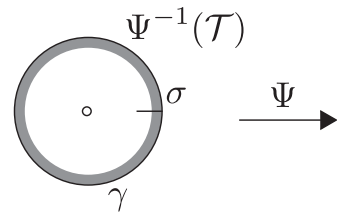

b)

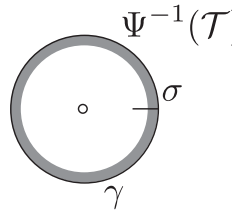

c)

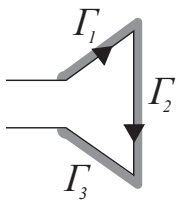

$5 \pi$
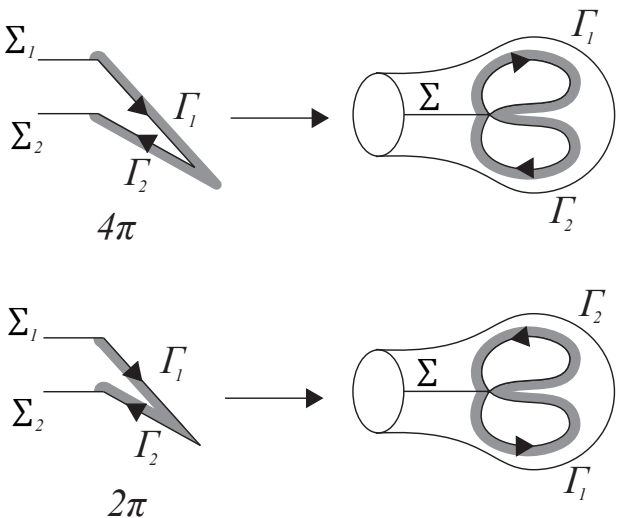

e)

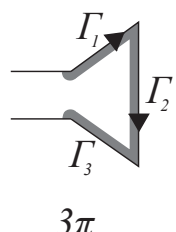

f)

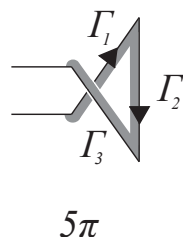

Figure 9. Half $\varepsilon$-tubular neighborhood $\mathcal{T}$ and cone angle. Drawings (a) and (b) are examples of $\Gamma \subset \mathbb{C}_{t}$ with 2 edges, having the same trace but with different half $\varepsilon$-tubular neighborhoods $\mathcal{T}$. After identification, we get a sphere minus a bouquet of two open disks. In (a) a double pole and two zeros are required (total cone angle $4 \pi+\pi+\pi$ ). In (b) a simple pole and two zeros are required (total cone angle $2 \pi+\pi+\pi$ ). Drawings (c)-(f) present $\Gamma \subset \mathbb{C}_{t}$ with 3 edges, the respective cone angle, determined by $\mathcal{T}$, is the odd multiple of $\pi$ indicated below. Hence the total cone angle is always an even multiple of $\pi$. See proof of Theorem 7.1 .

finite length with ends points at regular points $p_{1}, p_{2}$ of $\widetilde{X_{1}}$, and cutting $N$ along a segment of trajectory of $X_{2}$ with the same characteristics, use [49] construction 1 , pp. 242 or [66] 12.3 , pp. 57 . The connected sum $\widehat{\mathbb{C}} \cup N=M_{\mathfrak{g}}$ supports a new singular analytic vector field $\widetilde{X}$, having germs holomorphically equivalent to the original germs of $\widetilde{X}_{1}, X$ and at least two new simple poles $p_{1}$ and $p_{2}$.

Note that, the complex structure of $M_{\mathfrak{g}}$ in the above constructions, varies for one fixed initial germ $((\mathbb{C}, 0), X)$. However, this variation is hard to describe, in particular, we do not know if given $X$, all the complex structures for genus $\mathfrak{g} \geq 1$ can be attained by this construction.

Isolated singularities having admissible words is a natural case to study, we provide a simple statement.

Corollary 7.3. Let $((\mathbb{C}, 0), X)$ be a germ of a singular analytic vector field having an isolated singularity at 0 and determining an admissible word $\mathcal{W}_{X}$. There exists an extended singular analytic vector field $\widetilde{X}$ on $\widehat{\mathbb{C}}$, such that the germ $\widetilde{X}$ at $0 \in \widehat{\mathbb{C}}$ is 
holomorphically equivalent to the germ of $X$, with $\widetilde{X}$ having at most an additional pole and a finite number of simple zeros.

Proof. Recall that $e, h, p, \epsilon$ denote the number of elliptic $E$, hyperbolic $H$, parabolic $P$ and entire $\mathscr{E}$ letters (sectors) in $\mathcal{W}_{X}$. In fact, $\epsilon=0$ implies that $((\mathbb{C}, 0), X)$ is a zero or pole as in Table 2, in this case the result is elementary. By rule (A) of the admissible words the case $\epsilon=1$ is discarded. Hence we assume $\epsilon \geq 2$.

For each letter $H, E, P, \mathscr{E}$, we consider a pair $\left(S_{\iota}, X_{\iota}\right)$; Riemann surface (with boundary) and singular analytic vector field. The four pairs, a priori germs, are extended (see Figure 10) as follows.

i) $\left(S_{1}, X_{1}\right)$ is $\left(\overline{D(0,1)}, i \lambda_{2} z \frac{\partial}{\partial z}\right)$ giving rise to a semi-infinite height cylinder $S_{2 \pi /\left|\lambda_{2}\right|}^{1} \times(0, \infty)$; see Figure 10] a.

ii) $\left(S_{2}, X_{2}\right)$ is $\left(\overline{\mathbb{H}}^{2}, \frac{\partial}{\partial z}\right)$ giving rise to a half-plane; see Figure $10, \mathrm{~b}$.

iii) $\left(S_{3}, X_{3}\right)$ is $\left(\widehat{\mathbb{C}}, z \frac{\partial}{\partial z}\right)$ with a horizontal geodesic cut along $[1, \infty) \subset \widehat{\mathbb{C}}$ giving origin to two boundary geodesics of a cylinder $S_{2 \pi}^{1} \times \mathbb{R}$; see Figure [10]c and recall Table 2 ,

iv) $\left(S_{4}, X_{4}\right)$ is $\left(\overline{\mathbb{H}}^{2}, \mathrm{e}^{z} \frac{\partial}{\partial z}\right)$ giving rise to a half-logarithmic spiral; see Figures $10 \mathrm{~d}$ and 3 .

a)

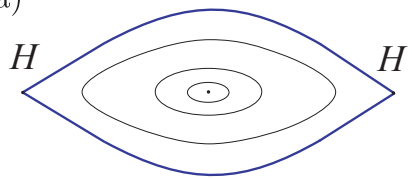

c)

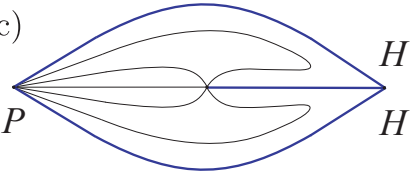

b)

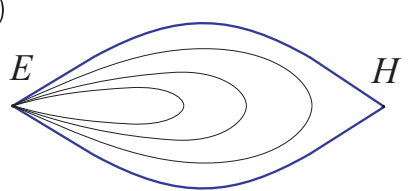

d)

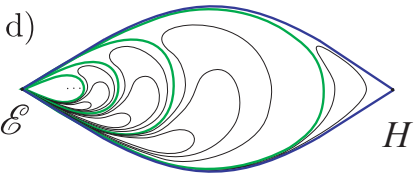

Figure 10. Word dualities. Four pairs $\left(S_{\iota}, X_{\iota}\right)$; a Riemann surface and a singular analytic vector field, provided with two geodesic boundary components. The left letters describe the original angular sectors of a germ $((\mathbb{C}, 0), X)$. The right letters describe the angular sectors at the corner point $q$. In cases (a), (c), simple zeros of $X_{\iota}$ appear inside of the respective $S_{\iota}$. See proof of Corollary 7.3.

Each $\left(S_{\iota}, X_{\iota}\right)$ has two marked points in their boundary, it will be convenient to rename these points as follows:

$0 \in \partial S_{\iota}$ as the left angular sector in Figure 10, $q \in \partial S_{\iota}$ as the right angular sector.

Summing up, we recognize that germs of (angular sectors or) letters $W_{\iota} \in$ $\{H, E, P, \mathscr{E}\}$ of $\mathcal{W}_{X}$ are realized at the marked points $0 \in \partial S_{\iota}$.

Moreover, in all four cases, the angular sectors $H$ at the marked points $q \in \partial S_{\iota}$ are well defined. 
We can identify $S_{\iota}$ topologically as one copy of $\left\{z \mid 0 \leq \arg (z) \leq \frac{2 \pi}{\ell}\right\} \subset \widehat{\mathbb{C}}$, where $\ell=(e+\epsilon+h+p)$.

More precisely, in the flat surface category, we perform the glueing of regions $\left(S_{\iota}, X_{\iota}\right)$, as in Corollary [5.11, following the cyclic pattern in $\mathcal{W}_{X}$, to recover at 0 the original germ of $((\mathbb{C}, 0), X)$.

In order to show that the isometric glueing is well defined, consider a germ representative of $X$ on a disk $D(0, \varepsilon)$. Let $l_{0} / 2>0$ be the minimal $g_{X}$-length of the collection of boundary geodesic trajectories $\left\{\sigma_{j}\right\}$ of the angular sectors of $\left\{W_{\iota}\right\}$ inside $D(0, \varepsilon)$ which have finite $g_{X}$-length. We can reduce the domain to a neighborhood $V \subset D(0, \varepsilon)$ such that new representative of $X$ satisfies that, the $g_{X}$-length of all the geodesic trajectories $\left\{\sigma_{j}\right\}$ in $V$ is exactly $l_{0}$.

Without loss of generality, we require that $\left(S_{\iota}, g_{X_{\iota}}\right)$ have finite boundary pieces of $g_{X_{L}}$-length $l_{0}$ in Figures 10,a and d, thus the isometric glueing is well defined.

The point $\infty \in S^{2}$ which originates from the marked right points $q \in \partial S_{\iota}$, defines a new word and additional zeros, following the rules:

$$
\begin{aligned}
& H \longleftrightarrow H \text { at } \infty \text { and an additional center } C \text { inside } S_{1}, \\
& E \longleftrightarrow H \text { at } \infty, \\
& P \longleftrightarrow H \text { at } \infty \text { and an additional source-sink } P \text { inside } S_{3}, \\
& \mathscr{E} \longleftrightarrow H \text { at } \infty .
\end{aligned}
$$

Where the left column means the original letters of $\mathcal{W}_{X}$, and the right one comes from considering the words $S_{\iota}$ at the new point $\infty \in S^{2}$.

Whence $\widetilde{X}$ on a new Riemann surface $M$ is obtained. The singularities of $\widetilde{X}$ at $\infty$ and at the zeros inside $S_{1}, S_{3}$ (Figure 10 a and c), determine conformal punctures in $M$, by the uniformization theorem we can recognize $M$ as the Riemann sphere $\widehat{\mathbb{C}}$. This produces the desired vector field $\widetilde{X}$ on $\widehat{\mathbb{C}}$ having $p+h$ simple zeros and a pole of multiplicity $1-(e+\epsilon+h+p) / 2 \leq-1$ (=0 means a nonsingularity), recalling that $(e+\epsilon+h+p) \geq 2$.

Remark 7.4 (The extended vector field $\widetilde{X}$ on $\widehat{\mathbb{C}}$ depends on the choice of the germ representative). Note that the numbers of sectors $e, \epsilon, h, p$ depend on the representative of the germ on $D(0, \varepsilon)$; in particular, recall Remark 5.18 where class 1 entire sectors "produce" new elliptic and hyperbolic sectors as the radius $\varepsilon \rightarrow 0$ in $(\mathbb{C}, 0)$.

The above construction is far from being optimal with respect to the number of additional zeros and poles; see Corollary 10.3 .

\section{Entire Structurally finite 1 -ORder $d$ VeCtor Fields $\mathcal{E}(d)$}

In $1994 \mathrm{~K}$. Hockett and S. Ramamurti studied the dynamics of vector fields of the form $\exp (P(z)) \frac{\partial}{\partial z}$ with $P(z)=z^{d}$ or $P(z)=a z^{2}+b z+c$, finding (verbatim [34]) "that the corresponding real flow has a 'bouquet' of $d$ flowers with a common root at the point at infinity and directions $k \pi / d, k$ odd". Furthermore, they conjectured that "the dynamics of the corresponding case when $P(z)$ is a general polynomial of degree $d$ is, up to conformal change of coordinates in the punctured plane, essentially the same as that of $P(z)=z^{d "}$.

We study the families of vector fields of the form $X(z)=\exp (P(z)) \frac{\partial}{\partial z}$ with $P(z)$ an arbitrary polynomial of degree $d$, obtaining a complete analytical description of these families. With this classification, we then show (amongst other things) that 
there are an infinite number of different topologies for $\mathfrak{R e}(X)$ in each case $d \geq 3$, a clear answer (in the negative) to the conjecture of K. Hockett and S. Ramamurti.

In order to do this we use the basic correspondence between vector fields $X$ and the global distinguished parameter $\Psi_{X}$ given by Lemma 2.6. thus the vector fields $X$ have a global flow-box (see $₫ 3.2$ and the commutative diagram (1.1)).

On the other hand structurally finite entire functions of type $(r, d)$,

$$
\Psi_{X}(z)=\int^{z} R(\zeta) \mathrm{e}^{P(\zeta)} d \zeta, \quad P, R \in \mathbb{C}[z],
$$

where $r=\operatorname{degree}(R), d=\operatorname{degree}(P)$, and their classification, are classically well known; see R. Nevanlinna [53], and M. Taniguchi's seminal work on structurally finite entire functions [67, 68]. With the above in mind, and using M. Taniguchi's notation, consider the following.

Definition 8.1. For $d \geq 1$, the family of entire structurally finite 1 -order $d$ vector fields is

$$
\mathcal{E}(d)=\left\{X(z)=\lambda \mathrm{e}^{P(z)} \frac{\partial}{\partial z} \mid P(z)=b_{0} z^{d}+b_{1} z^{d-1}+\ldots+b_{d}, \lambda b_{0} \neq 0\right\} .
$$

Certainly the parameters $\lambda$ and $b_{0}$ are redundant for $\mathcal{E}(d)$, but leaving both will simplify the computations needed for the proof of Theorem 8.24. Note that the group $A u t(\mathbb{C})$ is the largest 10 complex Lie group that acts holomorphically by pullback on $\mathcal{E}(d)$.

8.1. Revisiting the Riemann surface $\mathcal{R}_{X}$ associated to $X \in \mathcal{E}(d)$. In the following sections, sufficient elements that completely describe $\left(\mathcal{R}_{X}, \pi_{X, 2}^{*}\left(\frac{\partial}{\partial t}\right)\right)$ when we restrict ourselves to $X \in \mathcal{E}(d)$ are presented.

Remark 8.2. Recall diagram

(2.6)

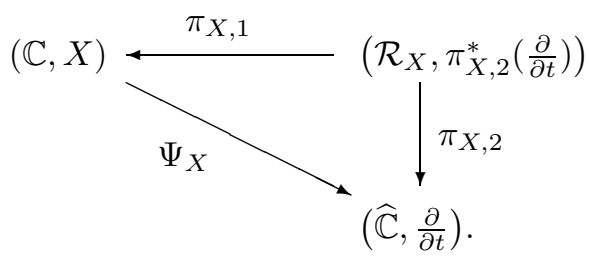

Using Lemma 2.7 for $X \in \mathcal{E}(d)$, it follows that $\pi_{X, 1}$ is a biholomorphism and $\pi_{X, 2}$ a ramified covering (with only logarithmic branch points, as will be shown shortly). In what follows, we shall use the abbreviated form $\mathcal{R}_{X}$ instead of the more cumbersome $\left(\mathcal{R}_{X}, \pi_{X, 2}^{*}\left(\frac{\partial}{\partial t}\right)\right)$.

As usual (see for instance [53, [58, 66], 67], 68]), once the branch points $\left\{b \in \mathcal{R}_{X}\right\}$ under $\pi_{X, 2}$ are determined, the Riemann surface $\mathcal{R}_{X}$ will be described as sheets $\mathbb{C}_{t} \backslash$ s suitable branch cuts $\}$ glued together in very specific ways. Since the sheets are elementary blocks, an accurate description is pertinent.

Definition 8.3. Let $\left\{a_{\mathrm{k}}\right\}_{\mathrm{k}=1}^{\mathrm{r}} \subset \mathbb{C}_{t}$ be a finite set of different points with $\mathrm{r} \geq 1$. A sheet is a copy of $\mathbb{C}_{t}$ with $r$ branch cuts $L_{\mathrm{k}}$; i.e. $\mathbb{C}_{t}$ is cut along horizontal segments

${ }^{10} \operatorname{Aut}(\mathbb{C})$ correspond to those $\operatorname{Aut}(\widehat{\mathbb{C}}) \cong P S L(2, \mathbb{C})$ that fix $\infty \in \widehat{\mathbb{C}}$. Furthermore, $X \in \mathcal{E}(d)$ are global objects on $\widehat{\mathbb{C}}$ so $A u t(\mathbb{C})$ is in fact the largest natural Lie group acting faithfully on $\mathcal{E}(d)$. 
$L_{\mathrm{k}}=\left[a_{\mathrm{k}}, \infty\right)$, remaining connected, but with $2 \mathrm{r}$ horizontal borders left there for further isometric glueing

$$
\mathbb{C}_{t} \backslash\left\{L_{\mathrm{k}}\right\}_{\mathrm{k}=1}^{\mathrm{r}} \cong\left[\mathbb{C}_{t} \backslash\left(\bigcup_{\mathrm{k}=1}^{\mathrm{r}}\left[a_{\mathrm{k}}, \infty\right)\right)\right] \bigcup_{\mathrm{k}=1}^{\mathrm{r}}\left\{\left[a_{\mathrm{k}}, \infty\right)_{+},\left[a_{\mathrm{k}}, \infty\right)_{-}\right\},
$$

where the sub-indices \pm refer to the obvious upper or lower boundary using $\mathfrak{I m}(t)$. We say that the height of $L_{\mathrm{k}}$ is $\mathfrak{I m}\left(a_{\mathrm{k}}\right)$. Left cuts and left borders $\left(\infty, a_{\mathrm{k}}\right]_{ \pm}$are also allowed, by notational simplicity the above equation is written using right cuts.

A diagonal of the sheet is

$$
\Delta_{\sigma \rho}=\overline{a_{\sigma} a_{\rho}} \subset \mathbb{C}_{t} \backslash\left\{L_{\mathrm{k}}\right\}_{\mathrm{k}=1}^{\mathrm{r}},
$$

i.e. it is the oriented straight line segment, in the sheet, starting at $a_{\sigma}$ and ending at $a_{\rho}$, here $\rho, \sigma \in\{1, \ldots, r\}$.

The upper drawings in Figure 11 provide examples of sheets and diagonals.

Since $X \in \mathcal{E}(d)$ has no poles or zeros, then there are no finitely ramified branch points in $\mathcal{R}_{X}$. Furthermore, logarithmic branch points project, via $\pi_{X, 2}$ of diagram (2.6), to the asymptotic values of $\Psi_{X}$; see 4.3 for the appropriate definitions.

Given an asymptotic value $a \in \widehat{\mathbb{C}}_{t}$, its preimages (under $\pi_{X, 2}$ ) in $\mathcal{R}_{X}$ can be any of the following: a regular point of $\mathcal{R}_{X}$ or a logarithmic branch point of $\mathcal{R}_{X}$. Both can coexist in the sense that $\pi_{X, 2}^{-1}(a)$ can contain regular points and logarithmic branch points on $\mathcal{R}_{X}$ all of which project via $\pi_{X, 2}$ to $a$.

The following lemma follows from M. Taniguchi's results; see [67] and 68].

Lemma 8.4 (Existence of logarithmic branch points). Let $\Psi: \mathbb{C}_{z} \rightarrow \widehat{\mathbb{C}}_{t}$ be a structurally finite entire function of type $(r, d)$. Then $\Psi^{-1}$ has $2 d$ direct singularities, no indirect singularities and $r$ critical values.

Moreover, the direct singularities correspond to d logarithmic branch points over $d$ finite asymptotic values $\left\{a_{\sigma}\right\} \subset \widehat{\mathbb{C}}_{t}$, and d logarithmic branch points over $\infty \in \widehat{\mathbb{C}}_{t}$.

Note that for $X \in \mathcal{E}(d), \Psi_{X}$ is a structurally finite entire function of type $(0, d)$. Further note that $\Psi_{X}$ is of 1 -order $d$. In fact, the hypothesis that $\Psi_{X}^{-1}$ has $d$ logarithmic branch points over $d$ finite asymptotic values, and $d$ logarithmic branch points over $\infty$, implies that $\Psi_{X}$ is of 1 -order $d$.

Proof of Lemma 8.4. Since $\infty \in \widehat{\mathbb{C}}_{z}$ is an isolated essential singularity of $\Psi$, a consequence of the theorem of Denjoy-Carleman-Ahlfors (see corollaries 1 and 3 in [12]) is that $\Psi$ has (at most) $2 d$ asymptotic values, and all the transcendental singularities $U_{\iota}$ of $\Psi^{-1}$ over each asymptotic value are logarithmic.

Moreover, since $\Psi$ is a structurally finite entire function of type $(r, d)$, then by Taniguchi's representation theorem (see theorem 1 of [67] or theorem 2.14 of [68]), it follows that:

1) $\Psi(z)=\int^{z} R(\zeta) \mathrm{e}^{P(\zeta)} d \zeta$ for $R, P \in \mathbb{C}[z]$ of degree $r$ and $d$, respectively.

2) The Riemann surface of $\Psi$ is constructed from $r$ quadratic blocks and $d$ expblocks by Maskit surgeries (corresponding to our isometric glueing Corollary 5.11).

Hence $\Psi$ has $r$ critical values (one for each quadratic block) and $\Psi^{-1}$ has $2 d$ logarithmic branch points (two for each exp-block). 
It follows immediately that $\Psi$ has: 1 -order $d, d$ finite asymptotic values (one for each exp-block), $d$ infinite asymptotic values (again one for each exp-block), and $r$ critical values (one for each quadratic block), all counted with multiplicity.

The last result tells us that for $X \in \mathcal{E}(d)$, the Riemann surface $\mathcal{R}_{X}$ has at least two logarithmic branch points that do not lie over the same asymptotic value in $\widehat{\mathbb{C}}_{t}$. In fact, as was pointed out by R. Nevanlinna ([53] pp. 291) "any many-sheeted surface has at least two logarithmic branch points that do not lie over one another". However, in the particular case of $X \in \mathcal{E}(d)$ for $d \geq 2$ the following result extends this: $\mathcal{R}_{X}$ has at least three logarithmic branch points that do not lie over the same asymptotic value in $\widehat{\mathbb{C}}_{t}$.

Lemma 8.5. Let $X \in \mathcal{E}(d)$, then $\Psi_{X}^{-1}$ has d logarithmic branch points over a single finite asymptotic value $a_{1} \in \mathbb{C}_{t}$ if and only if $d=1$.

Proof. $(\Leftarrow)$ When $d=1, \Psi_{X}^{-1}$ has only one logarithmic branch point over a finite asymptotic value. For a direct calculation, see (8.19).

$(\Rightarrow)$ We proceed by contrapositive. Suppose that $d \geq 2$, then $\Psi_{X}^{-1}$ has $d$ logarithmic branch points over $d$ finite asymptotic values (counted with multiplicity). If we assume that there is a single finite asymptotic value $a_{1} \in \mathbb{C}_{t}$, then it has multiplicity $d$. Thus $\mathcal{R}_{X}$ has $d \geq 2$ logarithmic branch points over the same finite asymptotic value $a_{1} \in \mathbb{C}_{t}$ and $d$ logarithmic branch points over $\infty \in \widehat{\mathbb{C}}_{t}$ and no other singular points. This space has $d$ connected components, while on the other hand, by Remark $8.2, \mathcal{R}_{X}$ is biholomorphic to $\mathbb{C}_{z}$, which of course consists of only one connected component.

Hence for $d \geq 2$ the number of distinct finite asymptotic values is $m \geq 2$.

8.1.1. Logarithmic branch points of $\mathcal{R}_{X}$. Given $X \in \mathcal{E}(d)$, the distinguished parameter $\Psi_{X}$ has exactly $d$ finite asymptotic values (counted with multiplicity). If we denote them by

$$
\left\{a_{j}\right\}_{j=1}^{m} \subset \mathbb{C}_{t} \text {, with multiplicities }\left\{\nu_{j}\right\}_{j=1}^{m} \text { and } \sum_{j=1}^{m} \nu_{j}=d,
$$

then $\pi_{X, 2}^{-1}\left(a_{j}\right)$ has one logarithmic branch point of $\mathcal{R}_{X}$ for each exponential tract associated to the finite asymptotic value $a_{j}$; see first and third lines in (8.8).

Moreover, $\pi_{X, 2}^{-1}(\infty)$ has $d$ logarithmic branch points since the asymptotic value $\infty$ has multiplicity $d$.

In other words, if $\alpha(\tau):[0, \infty) \rightarrow \mathbb{C}_{z}$ is an asymptotic path tending to $\infty \in \widehat{\mathbb{C}}_{z}$ associated to the asymptotic value $a \in\left\{a_{1}, \ldots, a_{m}, \infty\right\} \subset \widehat{\mathbb{C}}_{t}$, then we may assume that $\alpha(\tau)$ is contained in one exponential tract and

$$
\lim _{\tau \rightarrow \infty} \Psi_{X}(\alpha(\tau))=a .
$$

The exponential tracts will be in bijection with classes of asymptotic paths. With this in mind we shall identify each exponential tract simply by $\alpha$.

Thus, asymptotic values are actually pairs; $(\alpha, a)$, comprised of a value $a \in \widehat{\mathbb{C}}_{t}$ and the corresponding exponential tract $\alpha$. Since logarithmic branch points lie over asymptotic values, they are in correspondence with these pairs $(\alpha, a)$. In particular, the asymptotic value $a=\infty$ has $d$ different exponential tracts; each one corresponding to a logarithmic branch point over $\infty$. 
Remark 8.6. The logarithmic branch points associated to the isolated singularity at $\infty \in \widehat{\mathbb{C}}_{z}$, are not in fact in

$$
\mathcal{R}_{X} \subset \mathbb{C}_{z} \times \widehat{\mathbb{C}}_{t}
$$

Instead, see for instance [12], they lie on the non-Hausdorff closure $\overline{\mathbb{C}}_{z} \times \widehat{\mathbb{C}}_{t}$ of $\mathbb{C}_{z} \times \widehat{\mathbb{C}}_{t}$. Here,

$$
\overline{\mathbb{C}}_{z}:=((\widehat{\mathbb{C}} \times\{1\}) \sqcup(\widehat{\mathbb{C}} \times\{2\}) \sqcup \cdots \sqcup(\widehat{\mathbb{C}} \times\{2 d\})) / \sim
$$

is the sphere with $2 d$ infinities, that is the disjoint union of $2 d$ copies of the Riemann sphere $\widehat{\mathbb{C}}$ with the equivalence relation $\sim$, given by $(z, \sigma) \sim(z, \rho)$ for all $\sigma, \rho \in$ $\{1, \ldots, 2 d\}$ if $z \neq \infty$.

Recalling Lemma 8.4, we will denote the $2 d$ different infinities by $\left\{\infty_{\varsigma}\right\}_{\varsigma=1}^{2 d} \subset \overline{\mathbb{C}}_{z}$.

Remark 8.7. Numerical computations suggest (and the analytical computations found in $\$ 8.6$ for the cases $d=1,2,3$ show it in these cases), that $\lim _{\tau \rightarrow \infty} \Psi_{X}(\alpha(\tau))$ is either finite or infinite when we allow $\alpha(\tau)$ to stay in one of $2 d$ equally spaced angular sectors about $\infty \in \widehat{\mathbb{C}}_{z}$. In fact the limit alternates from finite to infinite to finite again as we go from one contiguous angular sector to another around $\infty$. This is in agreement with the above, in particular, that there are $2 d$ exponential tracts arising from the $2 d$ (counted with multiplicity) asymptotic values of $\Psi_{X}$ associated to the essential singularity at $\infty \in \widehat{\mathbb{C}}_{z}$.

Without loss of generality, by a renumbering in (8.5) if necessary, we may assume that the first $d$ indices of $\infty_{\varsigma} \in \overline{\mathbb{C}}_{z}$ are the ones related to the finite asymptotic values.

Given $a \in\left\{a_{1}, \ldots, a_{m}, \infty\right\} \subset \widehat{\mathbb{C}}_{t}$, we will denote by $\left(\infty_{\alpha}, a\right)$ the corresponding logarithmic branch point over a with associated exponential tract $\alpha$.

Since $a_{j}$ has multiplicity $\nu_{j}$, there will be $\nu_{j}$ asymptotic tracts for each distinct $a_{j}$. Moreover, the $d$ logarithmic branch points over the finite asymptotic values lie in $\overline{\mathbb{C}}_{z} \times \widehat{\mathbb{C}}_{t}$, however, slightly abusing notation they are to be thought in $\mathcal{R}_{X}$. We will denote them by

$$
b_{\sigma}=\left(\infty_{\sigma}, a_{\sigma}\right) \in \mathcal{R}_{X}, \text { for } \sigma \in\{1, \ldots, d\} .
$$

The corresponding $d$ logarithmic branch points over $\infty \in \widehat{\mathbb{C}}_{t}$ will be denoted by

$$
\left(\infty_{d+\sigma}, \infty\right) \in \mathcal{R}_{X}, \text { for } \sigma \in\{1, \ldots, d\} .
$$

In summary, for finite asymptotic values, the correspondence between indices is

$$
j \in \underbrace{1, \ldots, \nu_{1}}_{1}, \underbrace{\nu_{1}+1, \ldots, \nu_{1}+\nu_{2}}_{2}, \ldots, \underbrace{d-\nu_{m}+1, \ldots, d}_{m},
$$

where $\sigma$ enumerates the logarithmic branch points $b_{\sigma} \in \mathcal{R}_{X}$, thus $j=j(\sigma)$ enumerates the distinct finite asymptotic values $a_{j} \in \mathbb{C}_{t}$, and $\alpha=\alpha(\sigma)=\sigma$ enumerates the exponential tract:11 associated to the finite asymptotic values.

\footnotetext{
${ }^{11}$ We have opted to use a very simple enumeration of the exponential tracts suggested by Remark 8.7 but it is clear that a priori the enumeration is arbitrary.
} 
8.1.2. Global placement data. We also require information on where the different ramified branch points of $\pi_{X, 2}$ are in $\mathcal{R}_{X}$ and their interconnection on the surface.

Definition 8.8. Let $a_{\sigma}, a_{\rho} \in\left\{a_{1}, \ldots, a_{m}\right\} \subset \mathbb{C}_{t}$ be two distinct (finite) asymptotic values of $\Psi_{X}$ and consider the oriented straight line segment $\overline{a_{\sigma} a_{\rho}} \subset \mathbb{C}_{t}$. The inverse image $\pi_{X, 2}^{-1}\left(\overline{a_{\sigma} a_{\rho}}\right)=\left\{\Delta_{\vartheta \sigma \rho}\right\} \subset \mathcal{R}_{X}$ is a set consisting of an infinite number of copies of $\overline{a_{\sigma} a_{\rho}}$. For each segment $\Delta_{\vartheta \sigma \rho}$, let $\delta_{\vartheta \sigma \rho}=\pi_{X, 1}\left(\Delta_{\vartheta \sigma \rho}\right) \subset \overline{\mathbb{C}}_{z}$. We shall say that $\Delta_{\vartheta \sigma \rho} \subset \mathcal{R}_{X}$ is a diagonal, and that $a_{\sigma}$, $a_{\rho}$ share the same sheet $\mathbb{C}_{\Delta_{\vartheta \sigma \rho}} \backslash\{$ suitable branch cuts $\}$ in $\mathcal{R}_{X}$, when the interior 12 of $\delta_{\vartheta \sigma \rho}$ is in $\mathbb{C}_{z}$ and $\delta_{\vartheta \sigma \rho}$ has as its endpoints $\infty_{\sigma}, \infty_{\rho} \in \overline{\mathbb{C}}_{z}$.

The above coincides with Definition 8.3. Explicit examples of the use of diagonals appear in Examples 8.12 through 8.15 , and numerical computations in Theorem 8.24, equation (8.29).

Abusing notation, we will usually drop the index $\vartheta$ so a diagonal associated to $a_{\sigma}$ and $a_{\rho}$ will be denoted by $\Delta_{\sigma \rho}$, and sometimes by the segment $\overline{b_{\sigma} b_{\rho}}$, with $\pi_{X, 2}\left(b_{\sigma}\right)=a_{\sigma}$ and $\pi_{X, 2}\left(b_{\rho}\right)=a_{\rho}$. In this case the sheet shared by $a_{\sigma}$ and $a_{\rho}$ will be denoted by $\mathbb{C}_{\Delta_{\sigma \rho}} \backslash\{$ suitable branch cuts $\}$ in $\mathcal{R}_{X}$.

There is a relationship between the diagonals $\Delta_{\sigma \rho}$ of $\mathcal{R}_{X}$ and the semi-residues of $X$ (Definition 4.8). For $\Delta_{\sigma \rho}$ a diagonal associated to the finite asymptotic values $a_{\sigma}$ and $a_{\rho}$, note that $\delta_{\sigma \rho}=\pi_{X, 1}\left(\Delta_{\sigma \rho}\right)$ has as its endpoints $\infty_{\sigma}, \infty_{\rho} \in \overline{\mathbb{C}}_{z}$ and since $b_{\rho}, b_{\sigma} \in \mathcal{R}_{X}$, it follows that

$$
S\left(\omega_{X}, \infty_{\sigma}, \infty_{\rho}, \delta_{\sigma \rho}\right)=\int_{\delta_{\sigma \rho}} \omega_{X}=b_{\rho}-b_{\sigma}=\Delta_{\sigma \rho} .
$$

Lemma 8.9 (Existence of diagonals in $\mathcal{R}_{X}$ ). Assume that $d \geq 2$ and let $a_{\sigma}$ be any finite asymptotic value.

1) There exists at least a diagonal $\Delta_{\sigma \rho}$ associated to it.

2) By fixing a second (different) finite asymptotic value, $a_{\rho}$, the possible number of diagonals in the collection $\left\{\Delta_{\vartheta \sigma \rho}\right\} \subset \pi_{X, 2}^{-1}\left(\overline{a_{\sigma} a_{\rho}}\right)$ ranges from 0 to $d-1$.

Proof. Both assertions use that $\mathcal{R}_{X}$ is biholomorphic to $\mathbb{C}_{z}$; see Remark 8.2 .

Consider the logarithmic branch point $\left(\infty_{\sigma}, a_{\sigma}\right)$.

Suppose that there is no diagonal associated to it. This implies that $a_{\sigma}$ does not share a sheet, $\mathbb{C}_{t} \backslash\{$ suitable branch cuts $\}$, with any other finite asymptotic value. In other words, the only sheets, $\mathbb{C}_{t} \backslash\{$ suitable branch cuts $\}$, of $\mathcal{R}_{X}$ containing the branch point $\left(\infty_{\sigma}, a_{\sigma}\right)$ are of the form $\mathbb{C}_{t} \backslash\left\{L_{\sigma}\right\}$, for $L_{\sigma}=\left[a_{\sigma}, \infty\right)$; hence by the same arguments as in Lemma 8.5, $\mathcal{R}_{X}$ will have at least 2 connected components, leading to a contradiction.

Thus, there exists at least a diagonal associated to $\left(\infty_{\sigma}, a_{\sigma}\right)$, that is,

$$
\begin{aligned}
& \Delta_{\sigma \rho}=\overline{\left(\infty_{\sigma}, a_{\sigma}\right)\left(\infty_{\rho}, a_{\rho}\right)}, \\
& \quad \pi_{X, 2}\left(\left(\infty_{\sigma}, a_{\sigma}\right)\right)=a_{\sigma}, \pi_{X, 2}\left(\left(\infty_{\rho}, a_{\rho}\right)\right)=a_{\rho},
\end{aligned}
$$

for some $a_{\rho} \neq a_{\sigma}$.

\footnotetext{
${ }^{12}$ Since $\delta_{\vartheta \sigma \rho}$ is a path homeomorphic to $[a, b] \subset \mathbb{R}$, by the interior of $\delta_{\vartheta \sigma \rho}$ we mean the preimage, under the homeomorphism, of $(a, b)$.
} 
Moreover, the multiplicity $\nu_{j}=\nu_{j(\sigma)}$ of $a_{j}=a_{j(\sigma)}$ (see equation (8.4)), is the number of distinct preimages $\pi_{X, 2}^{-1}\left(a_{j}\right)=\left\{\left(\infty_{\sigma_{0}}, a_{j}\right), \ldots,\left(\infty_{\sigma_{0}+\nu_{j}-1}, a_{j}\right)\right\}$, for some $\sigma_{0} \in\left\{1, \nu_{1}+1, \ldots, d-\nu_{m}+1\right\}$, which are endpoints of a diagonal.

For assertion (2), the upper bound follows by considering a finite asymptotic value $a_{\sigma}$ of multiplicity one and a finite asymptotic value $a_{\rho}$ with multiplicity $d-1$, then the segment $\overline{a_{\sigma} a_{\rho}}$ lifts via $\pi_{X, 2}^{-1}$ to at most $d-1$ diagonals. See Examples 8.14, 8.15

8.2. Why is the description of $\mathcal{R}_{X}$, for $X \in \mathcal{E}(d)$ difficult? For $X$ be in $\mathcal{E}(d)$, we must completely describe, analytically, the Riemann surface $\mathcal{R}_{X}$. In order to do so, two combinatorial implicit obstacles are the following ones.

D.1. No canonical order can be given to $\left\{a_{\sigma}\right\}$ the asymptotic values of $\Psi_{X}$.

D.2. There is no preferred/canonical horizontal level 0 ,

$$
\mathbb{C}_{\Delta_{\sigma \rho}} \backslash\{\text { suitable branch cuts }\} \subset \mathcal{R}_{X},
$$

that is to be chosen to start the description of $\mathcal{R}_{X}$ as a combinatorial object.

8.3. $d$-configuration trees. We now introduce axiomatically certain trees capable of describing $\mathcal{R}_{X}$ from $X \in \mathcal{E}(d)$.

We will denote by $\widetilde{\mathbb{C}^{*}}=\left\{|z| \mathrm{e}^{i \arg (z)}\right\}$, the universal cover of $\mathbb{C}^{*}$, where $\arg (z)$ is the multivalued argument.

Definition 8.10. A $d$-configuration tree is a graph tree $\Lambda=\{V ; E\}$ with $d$ vertices

$$
V=\left\{\sigma:=\left(\infty_{\sigma}, a_{\sigma}\right)\right\}_{\sigma=1}^{d}
$$

where $\infty_{\sigma} \in \overline{\mathbb{C}}_{z}, a_{\sigma} \in \mathbb{C}_{t}$, and $d-1$ weighted edges

$$
E=\left\{\left(e_{\sigma \rho}, \tilde{\lambda}_{\sigma \rho}\right) \mid e_{\sigma \rho} \text { starts at }\left(\sigma \text { and ends at } \rho, \tilde{\lambda}_{\sigma \rho} \in \widetilde{\mathbb{C}^{*}}\right\} .\right.
$$

The following conditions must be satisfied.

For $d=1$ :

The 1 -configuration trees are $\left\{1=\left(\infty_{1}, a_{1}\right) ; \varnothing\right\}$.

For $d \geq 2$ :

1) (Existence of edges) There are no edges between vertices $\left(\sigma\right.$ and $\left(\rho\right.$ if $a_{\sigma}=a_{\rho}$.

2) (Weight of an edge) When an edge $e_{\sigma \rho}$ exists its associated weight is

$$
\widetilde{\lambda}_{\sigma \rho}=\left(a_{\rho}-a_{\sigma}\right) \mathrm{e}^{i 2 \pi K(\sigma, \rho)}=\left|a_{\rho}-a_{\sigma}\right| \mathrm{e}^{i \arg _{0}\left(a_{\rho}-a_{\sigma}\right)+i 2 \pi K(\sigma, \rho)} \in \widetilde{\mathbb{C}^{*}},
$$

where $K(\sigma, \rho) \in \mathbb{Z}$.

3) (Minimality condition) There are at least two vertices, say $(1)=\left(\infty_{1}, a_{1}\right)$ and $(=)\left(\infty_{\varrho}, a_{\varrho}\right)$ with $a_{1} \neq a_{\varrho}$, such that there is an edge $e_{1 \varrho}$ connecting them. The respective weight 13 satisfies

$$
\lambda_{1 \varrho}=a_{\varrho}-a_{1} \in \mathbb{C}^{*}, \quad \text { i.e. } K(1, \varrho)=0 .
$$

Several remarks regarding the complexity of Definition 8.10 are in order:

Remark 8.11. 1. The vertices $\odot$ will carry the information of asymptotic values including their exponential tracts. An advantage of the $\sigma$ notation is that it simplifies the $d$-configuration trees and their representation as in Figures 11 and 13. The enumeration by the index $\sigma$ is not canonical; see D.1 in 8.2

\footnotetext{
${ }^{13}$ In order to make it easier to describe the geometry of the Riemann surfaces $\mathcal{R}_{X}$, it will be convenient to sometimes use $\lambda_{\sigma \rho}$ instead of $\tilde{\lambda}_{\sigma \rho}$ to emphasize when the argument lies in $[0,2 \pi)$.
} 
2. A $d$-configuration tree can be thought of as a graph tree embedded in $\overline{\mathbb{C}}_{z} \times \widehat{\mathbb{C}}_{t}$.

3. The minimality condition (3) is not strictly required; it is a reflection of the fact that $\widetilde{\mathbb{C}^{*}}$ (and hence $\mathcal{R}_{X}$ ) has a logarithmic branch point over $0 \in \mathbb{C}_{t}$. This condition identifies a suitable copy of $\mathbb{C}^{*}$ in $\widetilde{\mathbb{C}^{*}}$ as the horizontal level 0 . The use of the minimality condition simplifies the notation of the $d$-configuration trees, in particular their $d$-skeleton (see Example 8.13 and Definition 8.19).

Example 8.12 (Examples of $d$-configuration trees, $d \leq 3$ ). 1 . For $d=1 ; \quad \Lambda=$ $\left\{1=\left(\infty_{1}, a_{1}\right) ; \varnothing\right\}$ as in Figure 11,a.

2. For $d=2 ; \quad \Lambda=\left\{(1)=\left(\infty_{1}, a_{1}\right)\right.$, (2) $\left.=\left(\infty_{2}, a_{2}\right) ;\left(\mathrm{e}_{12}, \lambda_{12}\right)\right\}$ as in Figure 11.b.

3. For $d=3$; let $a_{2}=1, a_{1}=\mathrm{e}^{i 2 \pi / 3}$ and $a_{3}=\mathrm{e}^{i 4 \pi / 3}$ be the cube roots of unity, $\lambda_{21}=a_{1}-a_{2}, \lambda_{13}=a_{3}-a_{1}$, and $\widetilde{\lambda}_{13}=\lambda_{13} \mathrm{e}^{i 4 \pi}$ (so $\left.K(1,3)=2\right)$. By agreeing that (1) $=\left(\infty_{1}, a_{1}\right),(2)=\left(\infty_{2}, a_{2}\right),(3)=\left(\infty_{3}, a_{3}\right)$, then the following

$$
\begin{aligned}
& \Lambda=\left\{(1),(2),(3) ;\left(e_{21}, \lambda_{21}\right),\left(e_{13}, \lambda_{13}\right)\right\}, \\
& \widetilde{\Lambda}=\left\{(1),(2),(3) ;\left(e_{21}, \lambda_{21}\right),\left(e_{13}, \widetilde{\lambda}_{13}\right)\right\}
\end{aligned}
$$

are two different 3-configuration trees; see Figure 11.c, d. Note that we have chosen an order for this example where the starting vertex for the enumeration is (2) and not (1) (see 8.2 D.1). Obviously, the minimality condition remains true with $\lambda_{21} \in \mathbb{C}^{*}$.

Example 8.13 (Usefulness of the minimality condition). Consider a $d$-configuration tree $\Lambda=\{V ; E\}, d \geq 2$, with vertices

$$
V=\left\{\left(\infty_{\sigma}, a_{\sigma}\right)\right\}_{\sigma=1}^{d}=\left\{\left(\infty_{1}, a_{1}\right),\left(\infty_{2}, a_{2}\right),\left(\infty_{3}, a_{3}\right), \ldots,\left(\infty_{d}, a_{d}\right)\right\}
$$

where the asymptotic values $\left\{a_{\sigma}\right\}_{\sigma=1}^{d} \subset \mathbb{C}_{t}$, have distinct imaginary parts, say $\mathfrak{I m}\left(a_{1}\right)<\cdots<\mathfrak{I m}\left(a_{d}\right)$, and edges given by

$$
E=\left\{\left(e_{\sigma \sigma+1}, \lambda_{\sigma \sigma+1}\right) \mid \lambda_{\sigma \sigma+1}=\left(a_{\sigma+1}-a_{\sigma}\right), \sigma \in\{1, \ldots, d-1\}\right\} .
$$

This $d$-configuration tree will correspond (as will be seen shortly) to a Riemann surface $\mathcal{R}_{X}$ with a logarithmic branch point over each $a_{\sigma}$.

For each $\sigma \in\{1, \ldots, d-1\}$, the collection of segments $\left\{\Delta_{\vartheta \sigma \sigma+1}\right\} \subset \pi_{X, 2}^{-1}\left(\overline{a_{\sigma} a_{\sigma+1}}\right)$ $\subset \mathcal{R}_{X}$ consists of exactly one diagonal and an infinite number of segments that are not diagonals.

Furthermore, since the weights $\lambda_{\sigma \sigma+1} \in \mathbb{C}^{*}$, all the $d-1$ diagonals share the same sheet $\mathbb{C}_{t} \backslash\left\{L_{\mathrm{k}}\right\}_{\mathrm{k}=1}^{d}$ in $\mathcal{R}_{X}$, (i.e. they all are on the same horizontal level of the $d$-skeleton, see Definition 8.19). See Figure 12, a.

Note that the minimality condition is trivially satisfied since all $\lambda_{\sigma \sigma+1} \in \mathbb{C}^{*}$.

On the other hand, if the minimality condition was not present in the definition, this example would change as follows.

A priori the horizontal level 0 (the starting sheet on $\mathcal{R}_{X}$ for the description of the $d$-skeleton) could contain the logarithmic branch point $\left(\infty_{1}, a_{1}\right)$ corresponding to the vertex (1) but not the diagonal (1)(2). However, since all the logarithmic branch points on $\mathcal{R}_{X}$ (corresponding to the vertices $\{(1), \ldots$, (d) $\}$ ) lie on the same sheet, then the weight $\lambda_{12}$ would have to include the information as to how many sheets in $\mathcal{R}_{X}$ are needed to go up or down around the logarithmic branch point $\left(\infty_{1}, a_{1}\right)$ before arriving at the sheet containing the other logarithmic branch points. 
a)

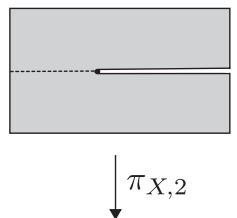

(1)

c)

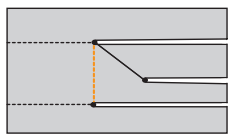

b)
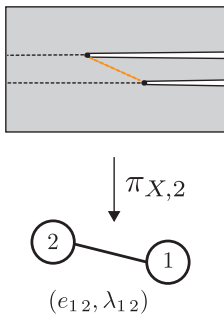

d)
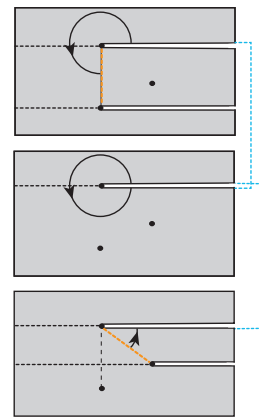

$\downarrow \pi_{X_{2}, 2}$

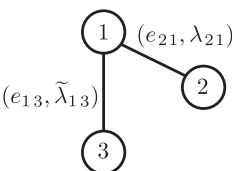

FiguRE 11. $d$-configuration trees $(d \leq 3)$ and the corresponding sheets in $\mathcal{R}_{X}$. (a) corresponds to Example 8.12.1. (b) corresponds to Example 8.12,2; here there is only one diagonal (1)(2) in a sheet $\mathbb{C}_{t} \backslash\left\{L_{\mathrm{k}}\right\}_{\mathrm{k}=1}^{2}$. (c) and (d) correspond to the two 3-configuration trees of Example 8.12 , 3, equations (8.12) and (8.13), respectively. Note that $\lambda_{13} \neq \widetilde{\lambda}_{13}$, since they differ in the argument by $4 \pi$. (c) has all three diagonals (denoted by the orange dotted lines) sharing one sheet $\mathbb{C}_{t} \backslash\left\{L_{\mathrm{k}}\right\}_{\mathrm{k}=1}^{3}$, even though the diagonal associated to (2)(3) is not used in the construction of the 3-configuration tree. (d) has only two diagonals $(\overline{(2)(3)}$ is not a diagonal) and each diagonal lies on its own sheet. The gray shaded regions correspond to the sheets $\mathbb{C}_{t} \backslash\{$ suitable branch cuts\} necessary to construct the soul of the $d$-configuration tree (see Definition 8.21 in text) for each example. Finite determination trajectories are denoted by black dotted lines. As will be seen in the proof of Theorem 8.16, cases (c) and (d) will each represent a Riemann surface: $\mathcal{R}_{X_{1}} \not \mathcal{R}_{X_{2}}$, for $X_{1}, X_{2} \in \mathcal{E}(3)$, with their associated $\pi_{X_{1}, 2}$ and $\pi_{X_{2}, 2}$ as in diagram (2.6). 
Thus $\lambda_{12}=\left(a_{2}-a_{1}\right) \mathrm{e}^{i 2 \pi K(1,2)}$ would in general have an argument not in $[0,2 \pi)$, that is, $K(1,2)$ assumes a value in $\mathbb{Z} \backslash\{0\}$. The $d$-skeleton would then have to include $K(1,2)$ vertical edges (with corresponding vertices) above or below the vertex associated to (1) of the horizontal subtree.

As was mentioned immediately after Definition 8.8 in $\$ 8.1$ for two fixed asymptotic values the number of corresponding diagonals that can occur as preimages of $\pi_{X, 2}$, ranges between 0 to $d-1$. It is now very simple to show this with $d-$ configuration trees.

Example 8.14. Consider a $d$-configuration tree $\Lambda=\{V ; E\}$, with vertices

$$
V=\left\{\left(\infty_{\sigma}, a_{\sigma}\right)\right\}_{\sigma=1}^{d}=\left\{\left(\infty_{1}, a_{1}\right),\left(\infty_{2}, a_{2}\right),\left(\infty_{3}, a_{3}\right), \ldots,\left(\infty_{d}, a_{d}\right)\right\}
$$

where the $d \geq 2$ distinct asymptotic values are $\left\{a_{\sigma}\right\}_{\sigma=1}^{d} \subset \mathbb{C}_{t}$, each with its corresponding exponential tract (note that each pair $\left(\infty_{\alpha(\sigma)}, a_{\sigma}\right)$ determines a distinct point in $\overline{\mathbb{C}}_{z} \times \widehat{\mathbb{C}}_{t}$ ), and edges given by

$$
E=\left\{\left(e_{1 \sigma}, \tilde{\lambda}_{1 \sigma}\right) \mid \tilde{\lambda}_{1 \sigma}=\left(a_{\sigma}-a_{1}\right) \mathrm{e}^{i 2 \pi(\sigma-2)}, \sigma \in\{2, \ldots, d\}\right\} .
$$

The corresponding $\mathcal{R}_{X}$ should have a logarithmic branch point over each $a_{\sigma}$.

For each $\sigma \in\{2, \ldots, d\}$, the collection of segments $\left\{\Delta_{\vartheta 1 \sigma}\right\} \subset \pi_{X, 2}^{-1}\left(\overline{a_{1} a_{\sigma}}\right) \subset \mathcal{R}_{X}$ consists of exactly one diagonal and an infinite number of segments that are not diagonals.

Furthermore, given $\sigma, \rho \in\{2, \ldots, d\}, \sigma \neq \rho$, there are no diagonals in the collection of segments $\left\{\Delta_{\vartheta \sigma \rho}\right\} \subset \pi_{X, 2}^{-1}\left(\overline{a_{\sigma} a_{\rho}}\right) \subset \mathcal{R}_{X}$.

In other words, there are exactly $d-1$ diagonals in $\mathcal{R}_{X}$. See Figure 12, b.

Hence, $\mathbb{C}_{t} \backslash\left\{a_{\sigma}\right\}_{\sigma=1}^{d}$ in Examples 8.13 and 8.14 are topologically equivalent; illustrating the obstacle considered in $98.2 \mathrm{D} .1$.

Example 8.15. Consider a $d$-configuration tree $\Lambda=\{V ; E\}$, with vertices

$$
V=\left\{\left(\infty_{\sigma}, a_{\sigma}\right)\right\}_{\sigma=1}^{d}=\left\{\left(\infty_{1}, a_{1}\right),\left(\infty_{2}, a_{2}\right),\left(\infty_{3}, a_{2}\right), \ldots,\left(\infty_{d}, a_{2}\right)\right\},
$$

with $d \geq 2$ and two distinct finite asymptotic values $a_{\sigma}=a_{2} \neq a_{1}$ for $\sigma \in\{2, \ldots, d\}$, the corresponding exponential tracts (once again each pair $\left(\infty_{\alpha(\sigma)}, a_{\sigma}\right)$ determines a distinct point in $\overline{\mathbb{C}}_{z} \times \widehat{\mathbb{C}}_{t}$ ), and edges given by

$$
E=\left\{\left(e_{1 \sigma}, \widetilde{\lambda}_{1 \sigma}\right) \mid \widetilde{\lambda}_{1 \sigma}=\left(a_{2}-a_{1}\right) \mathrm{e}^{i 2 \pi(\sigma-2)}, \sigma \in\{2, \ldots, d\}\right\} .
$$

The corresponding $\mathcal{R}_{X}$ should have a logarithmic branch point over $a_{1}$ and $d-1$ logarithmic branch points over $a_{2}$; the finite asymptotic values $a_{1}, a_{2}$ have multiplicities one and $d-1$.

The collection of segments $\left\{\Delta_{\vartheta} 1_{2}\right\} \subset \pi_{X, 2}^{-1}\left(\overline{a_{1} a_{2}}\right) \subset \mathcal{R}_{X}$ consists of exactly $d-1$ diagonals and an infinite number of segments that are not diagonals.

Once again there are exactly $d-1$ diagonals in $\mathcal{R}_{X}$. See Figure 12, c.

8.4. Natural equivalences in $\mathcal{E}(d)$. As a guide to the rest of this section, we remind the reader of the different categories that we study, and their natural equivalences in the family of vector fields $\mathcal{E}(d)$.

Combinatorial category. In 8.5 , the correspondence between classes of $d$-configuration trees $\left[\Lambda_{X}\right]$ and vector fields $X \in \mathcal{E}(d)$ is proved. 

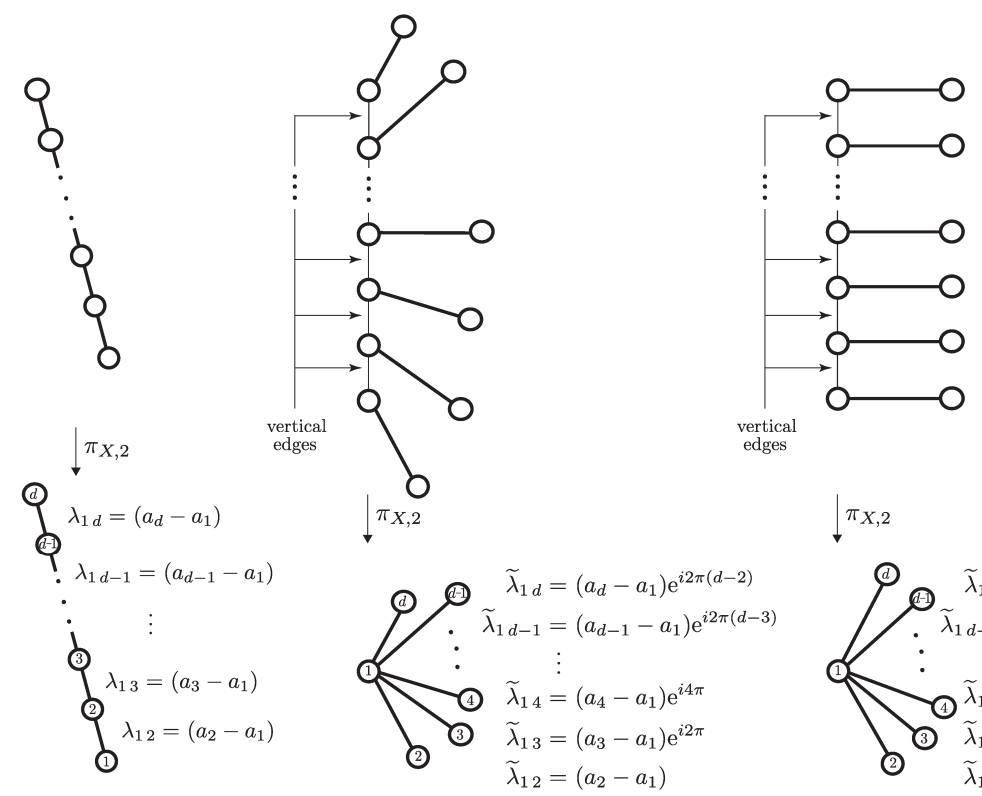

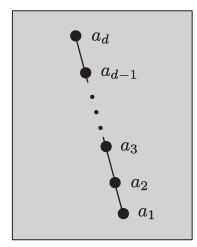

(a)
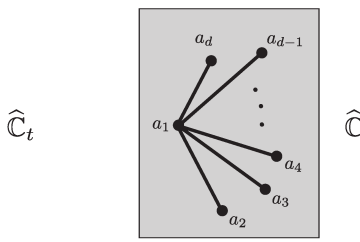

(b)
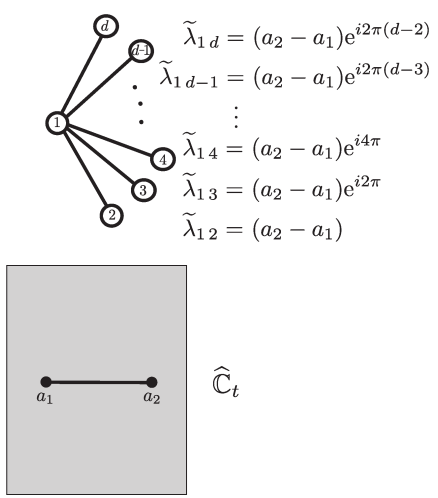

(c)

FIGURE 12. $d$-skeletons, $d$-configuration trees and the corresponding configurations $\mathbb{C}_{t} \backslash\left\{a_{\sigma}\right\}_{\sigma=1}^{d}$. Bottom row shows $\mathbb{C}_{t}$ with the finite asymptotic values and segments $\overline{a_{\sigma} a_{\rho}}$ (corresponding to the projection under $\pi_{X, 2}$ of the diagonals); the middle row shows the $d$-configuration trees with the weights $\widetilde{\lambda}_{\sigma \rho}$; the top row shows the $d$-skeleton (see Definition 8.19). (a) corresponds to Example 8.13: there is only one horizontal subtree, since the weights $\left\{\lambda_{\sigma \rho}\right\} \subset$ $\mathbb{C}^{*}$. (b) corresponds to Example 8.14; there are $d-1$ horizontal subtrees, each one consists of two vertices and a diagonal shown in black bold lines; by starting the traverse of the $d$-configuration tree from (2), each horizontal subtree is one level upwards of the previous one, as can be appreciated in the corresponding weights $\left\{\widetilde{\lambda}_{1 \sigma}\right\}$; since there are $d$ distinct finite asymptotic values the $d-1$ diagonals project onto $d-1$ segments in $\mathbb{C}_{t}$. (c) corresponds to Example 8.15 once again there are $d-1$ horizontal subtrees as in (b); the distinction is that there are only two finite asymptotic values $a_{1}, a_{2}$ with multiplicities one and $d-1$; thus the $d-1$ diagonals all project down to the segment $\overline{a_{1} a_{2}} \subset \mathbb{C}_{t}$. 
Complex analytic category. Since $\mathcal{E}(d)$ is a complex manifold and $A u t(\mathbb{C})$ is the largest complex Lie group that acts holomorphically by pullback on $\mathcal{E}(d)$, there will be a natural projection map

$$
\pi_{1}: \mathcal{E}(d) \longrightarrow \frac{\mathcal{E}(d)}{\operatorname{Aut}(\mathbb{C})}
$$

Note that a priori the quotient space $\mathcal{E}(d) / A u t(\mathbb{C})$ need not be a complex manifold. However, Theorem 8.16 provides an affirmative answer. This is the subject of 88.6 and 8.7

Flat singular surfaces category. The circle $S^{1}$ acts by $X \mapsto \mathrm{e}^{i \theta} X$ leaving invariant the respective flat metric $g_{X}$, hence we have another natural projection map

$$
\pi_{3}: \frac{\mathcal{E}(d)}{\operatorname{Aut}(\mathbb{C})} \longrightarrow \frac{\mathcal{E}(d)}{\operatorname{Aut}(\mathbb{C}) \times S^{1}}
$$

In this case the quotient space turns out to be a real analytic manifold, we examine this in 98.7

Topological category. The orientation preserving homeomorphism: 14 Homeo $(\mathbb{C})^{+}$ also act on $\mathcal{E}(d)$ by pullback on the singular foliations of the vector fields $\mathfrak{R e}(X)$, thus there is another natural projection map

$$
\pi_{2}: \frac{\mathcal{E}(d)}{\operatorname{Aut}(\mathbb{C})} \longrightarrow \frac{\mathcal{E}(d)}{\text { Homeo }(\mathbb{C})^{+}}
$$

The results concerning the image of $\pi_{2}$ are presented in 8.8

As a summary, the combinatorial, analytical, geometrical and topological classification of $\mathcal{E}(d)$ is expressed in diagram (1.2).

\subsection{Description of the family $\mathcal{E}(d)$ via combinatorial scheme.}

Theorem 8.16 ( $d$-configuration trees as parameters for $\mathcal{E}(d))$.

1) Entire structurally finite 1-order d vector fields are in one-to-one correspondence with classes of $d$-configuration trees, i.e.

$$
\mathcal{E}(d) \cong\left\{\left[\Lambda_{X}\right] \mid \Lambda_{X} \text { is a d-configuration tree }\right\} .
$$

2) The family $\mathcal{E}(d)$ of entire structurally finite 1 -order $d$ vector fields is a complex manifold of dimension $d+1$.

The equivalence classes $\left[\Lambda_{X}\right]$ of $d$-configuration trees will be described in Remark 8.5 .3 .

Proof. Recalling Lemma 2.6 to prove (1) of the theorem, it is enough to show that the classes of $d$-configuration trees are in one-to-one correspondence with the Riemann surfaces $\mathcal{R}_{X}$ for $X \in \mathcal{E}(d)$.

\footnotetext{
${ }^{14}$ Once again $H$ omeo $(\mathbb{C})^{+}$correspond to those orientation preserving homeomorphisms of $\widehat{\mathbb{C}}$, $\operatorname{Homeo}(\widehat{\mathbb{C}})^{+}$, that fix $\infty \in \widehat{\mathbb{C}}$.
} 
8.5.1. First part of the proof: Given $X \in \mathcal{E}(d)$ and its Riemann surface $\mathcal{R}_{X}$, we construct a $d$-configuration tree $\Lambda_{X}$.

Case $d=1$. Let $b_{1} \in \mathcal{R}_{X}$ be such that $\pi_{X, 2}\left(b_{1}\right)=a_{1}$ (see Remark 8.6), then $\left\{\left(\infty_{1}, a_{1}\right) ; \varnothing\right\}$ is the 1 -configuration tree determined by the finite asymptotic value $a_{1}$. For the explicit analytic expression of $X$ see Theorem 8.24, and Figures 1 and 3.

Case $d \geq 2$. From $\mathcal{R}_{X}$, we shall identify the elements that will enable us to describe it as a $d$-configuration tree.

1. Vertices of $\Lambda_{X}$. Following equations $(8.6)$, (8.8), let the vertices be

$$
V=\left\{\sigma=\left(\infty_{\sigma}, a_{\sigma}\right)\right\}_{\sigma=1}^{d} .
$$

2. Edges of $\Lambda_{X}$. It is natural to consider that the diagonals (Definition 8.8) should be the edges, however there is a caveat (see (b)).

a) Let the edge $e_{\sigma \rho}$ between $(\sigma$ and $(\rho$ exist whenever the segment

$$
\overline{\left(\infty_{\sigma}, a_{\sigma}\right)\left(\infty_{\rho}, a_{\rho}\right)} \text { is a diagonal } \Delta_{\sigma \rho} .
$$

By Lemma 8.9, there is always at least one edge associated to every vertex and there is always a path of edges that connects any two vertices.

b) If by considering (a) we obtain a cycle in the corresponding graph, choose any one edge of said cycle and remove it; no information is lost. See for example Figure 11. c where the edge (1)(3) is removed to avoid a cycle.

c) Thus the edges will be in 1-1 correspondence with the set of diagonals described in (a) and (b).

This provides us with a (nonweighted) tree that has $d$ vertices and $d-1$ edges.

3. Weights of $\Lambda_{X}$. In order to assign the corresponding weights to the edges, we will need to traverse the tree. However, there is no canonical way of doing so (recall 8.2.D.1), hence the notation will get inherently complicated. An explicit example, 8.17 to help understand the assignment of the weights is provided; the reader may follow along using Figure 13 .

Example 8.17 (Combinatorial aspects from sheets of an $\mathcal{R}_{X}$ to its 6 -configuration tree $\left.\Lambda_{X}\right)$. Let $\mathcal{R}_{X}$ be a simply connected Riemann which is described as sheets (Definition 8.3) glued together in the following very specific way (see Figure 13):

There are six logarithmic branch points $\left\{\sigma=\left(\infty_{\sigma}, a_{\sigma}\right)\right\}$ over six distinct finite asymptotic values $\left\{a_{\sigma}\right\}_{\sigma=1}^{6}$ of multiplicity one.

In addition, we assume that for $\{\sigma\}:=\{1,2,4\} \subset\{1, \ldots, 6\}$, a sheet

$$
\mathbb{C}_{t} \backslash\left\{L_{\sigma}=\left[a_{\sigma}, \infty\right)\right\}_{\sigma=1,2,4} \subset \mathcal{R}_{X} \text { exists. }
$$

The sheet determines two edges and diagonals $\Delta_{12}, \Delta_{24}$ whose extremes are the corresponding vertices (1), (2), (4) of $\Lambda_{X}$. Since the vertices share the same sheet, we define the weights as

$$
\lambda_{12}=a_{2}-a_{1}, \quad \lambda_{24}=a_{4}-a_{2} \quad \in \mathbb{C}^{*} .
$$

It is natural to say that the sheet (8.14) determines a horizontal subtree of $\Lambda_{X}$, corresponding to the horizontal level 0 (since it contains the starting vertex of the description of $\mathcal{R}_{X}$ as a combinatorial object). 
We assume the analogous in $\mathcal{R}_{X}$ for a second set $\{\sigma\}:=\{2,3,5,6\}$, a sheet

$$
\mathbb{C}_{t} \backslash\left\{L_{\sigma}=\left[a_{\sigma}, \infty\right)\right\}_{\sigma=2,3,5,6} \subset \mathcal{R}_{X} \text { exists. }
$$

It determines the respective vertices, edges and weights in $\Lambda_{X}$, as well as another horizontal subtree of $\Lambda_{X}$.

Furthermore, $\mathcal{R}_{X}$ contains three sheets of type $\mathbb{C}_{t} \backslash\left\{L_{2}=\left[a_{2}, \infty\right)\right\}$, forming a finite helicoid (Definition 5.4), and glued together to the sheets (8.14) and (8.15) in such way that

$$
\widetilde{\lambda}_{23}=\left(a_{3}-a_{2}\right) \mathrm{e}^{i 2 \pi K(2,3)} \in \widetilde{\mathbb{C}^{*}},
$$

where $2 \pi K(2,3)=-6 \pi$ is the argument (measured inside $\mathcal{R}_{X}$ ) between the sheets containing the diagonals $\Delta_{12}$ and $\Delta_{23}$ in the sheets (8.14), 8.15), respectively. In other words, the sheet (8.15) containing $\Delta_{23}$ is $K(2,3)=-3$ levels (downwards) from the sheet (8.14) containing $\Delta_{12}$.

This finishes the construction of $\Lambda_{X}$ from $\mathcal{R}_{X}$.

Note that the sheets (8.14), 8.15) also have half-logarithmic spirals (see Definition 5.5) glued to them. The sheets of $\mathcal{R}_{X}$ inside these spirals (red vertices in Figure 13) do not concern $\Lambda_{X}$. In the second part of the proof of Theorem 8.16. recognizing $\mathcal{R}_{X}$, for $X \in \mathcal{E}(6)$, will become clear.

Assignment of the weights.

a) We start to traverse the tree at a leaf (a vertex of degree 1), without loss of generality we will assume that said leaf is (1), and the edge corresponding to the leaf is $e_{1 \varrho}$. That is $e_{1 \varrho}$ connects the vertex (1) and @). We define the weight

$$
\lambda_{1 \varrho}=a_{\varrho}-a_{1} \in \mathbb{C}^{*} \text { i.e. } K(1, \varrho)=0,
$$

hence (1),@ share the same sheet in $\mathcal{R}_{X}$, thus achieving the minimality condition. This exhausts the edges containing the leaf (1).

b) Now standing at the vertex (@), we can choose a vertex $\sigma$, with $\sigma \neq 1, \varrho$, such that the edge $e_{\varrho \sigma}$ exists. The associated weight is defined as in (8.11) by

$$
\tilde{\lambda}_{\varrho \sigma}=\left(a_{\sigma}-a_{\varrho}\right) \mathrm{e}^{i 2 \pi K(\varrho, \sigma)}=\left|a_{\sigma}-a_{\varrho}\right| \mathrm{e}^{i \arg _{0}\left(a_{\sigma}-a_{\varrho}\right)+i 2 \pi K(\varrho, \sigma)},
$$

where $2 \pi K(\varrho, \sigma)$ is the argument (measured inside $\mathcal{R}_{X}$ ) between the sheets containing the diagonals $\Delta_{1 \varrho}$ and $\Delta_{\varrho \sigma}$.

Note that geometrically $K(\varrho, \sigma) \in \mathbb{Z}$ corresponds to the number of sheets in $\mathcal{R}_{X}$ that separate $\Delta_{1 \varrho}$ and $\Delta_{\varrho \sigma}$.

As is usual (for instance on the Riemann surface of the logarithm), going around a ramified branch point counterclockwise makes us go "upwards" on the ramified surface and hence the number that separates the sheets is positive. Similarly, going around the ramified branch point clockwise makes us go "downwards" on the ramified surface.

c) Continue the construction as in (b) for all the edges that contain the vertex (@), assigning the corresponding weight just as before. This exhausts the edges containing vertex @.

d) Standing at the vertex $\left(\sigma\right.$ of the edge $e_{\varrho} \sigma$, we can now choose a vertex $(\rho)$, not equal to the ones already traversed, and such that the edge $e_{\sigma \rho}$ exists. Once again, define the weight, as in (8.11) by

$$
\tilde{\lambda}_{\sigma \rho}=\left(a_{\rho}-a_{\sigma}\right) \mathrm{e}^{i 2 \pi K(\sigma, \rho)}=\left|a_{\rho}-a_{\sigma}\right| \mathrm{e}^{i \arg _{0}\left(a_{\rho}-a_{\sigma}\right)+i 2 \pi K(\sigma, \rho)},
$$

where $2 \pi K(\sigma, \rho)$ is the argument between the sheets containing $\Delta_{\varrho \sigma}$ and $\Delta_{\sigma \rho}$. 


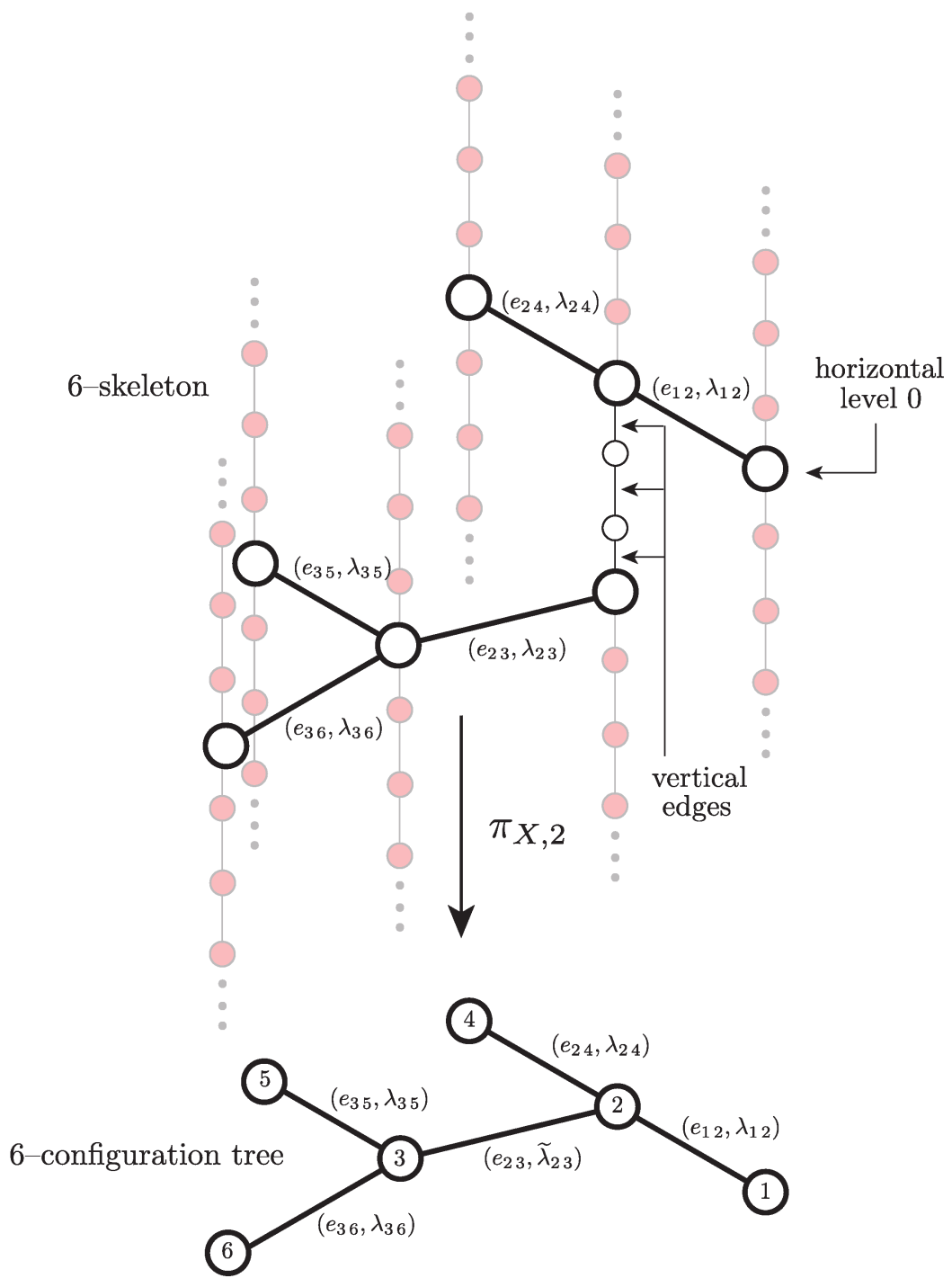

Figure 13. A 6-configuration tree $\Lambda_{X}$ and its 6-skeleton (corresponding to Example 8.17). In the case pictured, the weights $\lambda_{12}, \lambda_{24} \in \mathbb{C}^{*}$, i.e. have arguments in $[0,2 \pi)$ so the vertices (1), (2), and (4) together with the edges $\left(e_{12}, \lambda_{12}\right)$ and $\left(e_{24}, \lambda_{24}\right)$ give rise to the horizontal level 0 . On the other hand, $\widetilde{\lambda}_{23} \in \widetilde{\mathbb{C}}^{*}$ has argument in $[-6 \pi,-4 \pi)$, while $\lambda_{35}, \lambda_{36} \in \mathbb{C}^{*}$, hence the subtree, in the 6-skeleton, formed by the vertices (2), (3), (5) and (6) is 3 levels downwards from the horizontal level 0 . Note that the weight $\widetilde{\lambda}_{23}$ gives origin to $\lambda_{23} \in \mathbb{C}^{*}$, while $\lambda_{12}, \lambda_{24}, \lambda_{35}$ and $\lambda_{36}$ all lie in $\mathbb{C}^{*}$. The red shaded elements in the figure are a representation of the semi-infinite helicoids that are glued to the soul of $\Lambda_{X}$ to obtain the surface $\mathcal{R}_{X}$. 
e) Continuing in this way until all the vertices are exhausted, we assign weights to all the edges.

We have then constructed a $d-$-configuration tree $\Lambda_{X}$ associated to $X$.

Remark 8.18. Note that there might be other $d$-configuration trees associated to $X$ since in 8.5 .12 .b there could be a choice when assigning the edges. This will be addressed in 88.5 .3 .

8.5.2. Second part of the proof: Given a d-configuration tree $\Lambda_{X}$, we construct a simply connected Riemann surface $\mathcal{R}_{X}$ with $d$ logarithmic branch points, i.e. $X \in \mathcal{E}(d)$.

The construction will proceed in two steps, we will first construct an auxiliary tree, the $d$-skeleton (see definition below), describing the embedding of $\Lambda_{X}$ in $\overline{\mathbb{C}}_{z} \times \widehat{\mathbb{C}}_{t}$. As a second step, from the $d$-skeleton we will construct a simply connected Riemann surface $\mathcal{R}_{X}$ with $d$ logarithmic branch points.

The $d$-skeleton will contain the same information as $\Lambda_{X}$, with the disadvantage that it is more cumbersome to express, but with the advantage that it will allow us to clearly identify the equivalence classes of $\Lambda_{X}$.

1. Construction of the $d$-skeleton. We extend a $d$-configuration tree

$$
\Lambda_{X}=\left\{\left\{\sigma=\left(\infty_{\sigma}, a_{\sigma}\right)\right\}_{\sigma=1}^{d} ;\left\{\left(e_{\sigma \rho}, \tilde{\lambda}_{\sigma \rho}\right)\right\}\right\},
$$

by the following procedure (see Figure 13).

First recall that for each weighted edge, $\left(e_{\sigma \rho}, \widetilde{\lambda}_{\sigma \rho}\right)$, that starts at $\odot$ and ends at ( ) , the weight can be expressed as

$$
\widetilde{\lambda}_{\sigma \rho}=\lambda_{\sigma \rho} \mathrm{e}^{i 2 \pi K(\sigma, \rho)},
$$

with $\lambda_{\sigma \rho} \in \mathbb{C}^{*}$ and $K(\sigma, \rho) \in \mathbb{Z}$.

I) For each vertex $\sigma=\left(\infty_{\sigma}, a_{\sigma}, \infty\right)$, of the original $\Lambda_{X}$ :

a) Let

$$
K_{\max }=\max _{\rho}\{0, K(\sigma, \rho)\} \quad \text { and } \quad K_{\min }=\min _{\rho}\{0, K(\sigma, \rho)\},
$$

where the maximum and minimum are taken over all the edges that start at $(\sigma$ and end at $(\rho$ ).

b) Construct a vertical tower associated to $\sigma$ consisting of exactly $\left(K_{\max }-\right.$ $\left.K_{\min }+1\right)$ copies of the vertex $\sigma$ joined by $\left(K_{\max }-K_{\min }\right)$ vertical edges (without weights). We shall assign, consecutively, to each vertex of the vertical tower a level: an integer starting at $K_{\min }$ and ending at $K_{\max }$. Call the increasing direction $u p$ and the decreasing direction down.

At this point the vertical tower will have vertices of degree 2, except for the vertices at levels $K_{\min }$ and $K_{\max }$ which have degree 1.

II) We proceed with the construction.

a) Replace all the vertices with their associated vertical tower.

b) The edges require a little more finesse: The edge, $\left(e_{\sigma \rho}, \widetilde{\lambda}_{\sigma \rho}\right)$, is to end at the level 0 vertex of the vertical tower associated to $(\rho$ ). Furthermore, it should start at the level $K(\sigma, \rho)$ vertex of the vertical tower associated to $\sigma$.

c) Finally, replace the weights $\tilde{\lambda}_{\sigma \rho}$ by $\lambda_{\sigma \rho}$.

Definition 8.19. We shall call this tree the $d$-skeleton of $\Lambda_{X}$. 
The $d$-skeleton has the following properties (see Figure 13):

A) All edges that are not vertical are of the form $\left(e_{\sigma \rho}, \lambda_{\sigma \rho}\right)$ with $\lambda_{\sigma \rho} \in \mathbb{C}^{*}$. We shall call these horizontal edges.

B) Consider two horizontal edges $\left(e_{\sigma \rho}, \lambda_{\sigma \rho}\right)$ and $\left(e_{\rho \tau}, \lambda_{\rho \tau}\right)$ on the $d$-skeleton that share the vertex $\left(\rho\right.$ in the original $d$-configuration tree $\Lambda_{X}$. We shall say that the horizontal edge $\left(e_{\rho \tau}, \lambda_{\rho \tau}\right)$ is $K(\rho, \tau)$ levels upwards or downwards of the edge $\left(e_{\sigma \rho}, \lambda_{\sigma \rho}\right)$ in the $d$-skeleton depending on whether $K(\rho, \tau)$ is positive or negative, respectively. In case that $K(\rho, \tau)=0$ we shall say that the edges share the same horizontal level.

C) The $d$-skeleton has horizontal subtrees formed by the horizontal edges on the same horizontal level. From the minimality condition the weight $\lambda_{12} \in \mathbb{C}^{*}$, hence the subtree containing $\left(e_{12}, \lambda_{12}\right)$ will correspond to the horizontal level 0 subtree of the $d$-skeleton.

D) By collapsing the vertical edges of the $d$-skeleton we recover the original $d-$ configuration tree, losing the information of the argument of the original weights $\widetilde{\lambda}_{\sigma \rho}$. This collapsing of the $d$-skeleton can also be thought off as the projection $\pi_{X, 2}$ to the original $d$-configuration tree; see Figure 13 .

2. Construction of $\mathcal{R}_{X}$ from the $d$-skeleton. For simplicity, let us first assume that the $m$ distinct values $\left\{a_{j(\sigma)}\right\}_{j(\sigma)=1}^{m} \subset\left(\mathbb{C}, \frac{\partial}{\partial t}\right)$ arising from the vertices $\sigma=$ $\left(\infty_{\sigma}, a_{\sigma}\right)$, lie on $m$ different horizontal trajectories, i.e. $\mathfrak{I m}\left(a_{1}\right)<\cdots<\mathfrak{I m}\left(a_{m}\right)$.

There are two types of vertices on the $d$-skeleton; those that do not share horizontal edges (these are the vertices with vertical edges, i.e. on the vertical towers) and those that share horizontal edges (the vertices that belong to a horizontal subtree).

Remark 8.20. Combinatorial aspects of a sheet, from the $d$-skeleton to $\mathcal{R}_{X}$. We recall Definition 8.3 .

Case 1. If we have a vertex $(\sigma$ with only vertical edges attached to it (there are only two such vertical edges), then we obtain a sheet $\mathbb{C}_{t} \backslash\left\{L_{\sigma}\right\}$ with only one $L_{\sigma}$. Note that the two boundaries $\left[a_{\sigma}, \infty\right)_{ \pm}$correspond to the vertical edges.

Case 2. If we have a horizontal subtree in the $d$-skeleton, say with vertices $\sigma_{\ell}$, then we obtain a sheet $\mathbb{C}_{t} \backslash\left\{L_{\sigma_{\ell}}\right\}_{\ell}$. Once again the edges $e_{\sigma \rho}$ correspond to the diagonals $\Delta_{\sigma \rho} \subset \mathbb{C}_{t} \backslash\left\{L_{\sigma_{\ell}}\right\}_{\ell}$.

We proceed with the construction.

a) Replace each vertex 15 that does not share a horizontal edge with a copy of $\mathbb{C}_{t} \backslash L_{\sigma}$.

b) Given a horizontal subtree with $s$ vertices, let $v_{\ell}$, for $\ell \in\{1, \ldots, s\}$ be its vertices. Denote by $\sigma_{\ell}$ the vertex, of the original $d$-configuration tree, to which the vertex $v_{\ell}$ projects down to. Replace the given horizontal subtree with a copy of

$$
\mathbb{C}_{t} \backslash\left\{L_{\sigma_{\ell}}\right\}_{\ell=1}^{s},
$$

\footnotetext{
${ }^{15}$ Recall that all the vertices of the $d$-skeleton are either the original vertices $\sigma$ of the original $d$-configuration tree, or copies of them. Thus any vertex in the $d$-skeleton projects to a unique vertex on $\Lambda_{X}$.
} 
where each $L_{\sigma_{\ell}}$ is the horizontal branch cut associated to the vertex $\sigma_{\ell \ell}$. Since all the values $\left\{a_{\sigma_{\ell}}\right\}$ lie on different horizontal trajectories of $\frac{\partial}{\partial t}$, then none of the horizontal branch cuts $L_{\sigma_{\ell}}$ intersect in $\mathbb{C}_{t}$.

c) Continue as in (b) for every horizontal subtree.

d) Note that we obtain stacked copies of $\mathbb{C}_{t} \backslash L_{\sigma}$ and $\mathbb{C}_{t} \backslash\left\{L_{\sigma_{\ell}}\right\}_{\ell=1}^{s}$, but they retain their relative position with respect to the $d$-skeleton, by the fact that we still have not removed the vertical edges of the $d$-skeleton.

e) On each vertical tower, say the one associated to the vertex $(\sigma$, glue together, using Corollary 5.11, the horizontal branch cuts by alternating the boundaries of $\mathbb{C}_{t} \backslash L_{\sigma}$, so as to form finite helicoids (recall Definition 5.4) glued together over the vertex $(\sigma)$, making sure that all the finite helicoids go upwards when turning counterclockwise around the vertex. Note that these finite helicoids replace the vertical towers in the $d$-skeleton. Erase the vertical edges of the $d$-skeleton.

We obtain a simply connected surface constructed from the $d$-skeleton that has as boundary horizontal branch cuts $\left[a_{\sigma}, \infty\right)_{-} \cup\left[a_{\sigma}, \infty\right)_{+}$associated to the values $\left\{a_{\sigma}\right\}_{\sigma=1}^{d} \subset \mathbb{C}_{t}$.

If some of the values $\left\{a_{\sigma}\right\}_{\sigma=1}^{d} \subset\left(\mathbb{C}, \frac{\partial}{\partial t}\right)$ arising from the vertices $\sigma$, lie on the same horizontal trajectory of $\frac{\partial}{\partial t}$, then by Sard's theorem there is a small enough angle $\theta>0$ such that the set of values $\left\{a_{\sigma}\right\} \subset\left(\mathbb{C}, \mathrm{e}^{i \theta} \frac{\partial}{\partial t}\right)$ lie on $m$ different trajectories of $\mathrm{e}^{i \theta} \frac{\partial}{\partial t}$ (in fact any small enough angle $\theta \neq 0$ will suffice). Proceed with the construction (a)-(e) as above but using $\mathrm{e}^{i \theta} L_{\sigma}$ instead of $L_{\sigma}$ for the construction. Note that for small enough $\theta>0$ all the surfaces obtained are homeomorphic. Finally, let $\theta \rightarrow 0^{+}$and consider the limiting surface 16 .

Definition 8.21. We shall call this surface the soul of the $d$-configuration tree $\Lambda_{X}$.

In Figure 11 we can observe the soul of $d$-configuration trees for $d=1,2,3$. An example of the soul of a 4-configuration tree is in Figure 19. Applying the construction (a)-(e) to the 6-skeleton of Figure 13 one obtains the soul of the 6-configuration of Example 8.17

Finally, glue a semi-infinite helicoid to each of the $2 d$ boundaries of the soul of $\Lambda_{X}$ to obtain a simply connected Riemann surface $\mathcal{R}_{X}$ that is many sheeted with $d$ logarithmic branch points over finite asymptotic values. In fact, this surface is realized via Maskit surgeries with $d$ exp-blocks, so, by [67, 68, it corresponds to a function

$$
\Psi_{X} \in S F_{r, d}=\left\{\int_{z_{0}}^{z} R(\zeta) \mathrm{e}^{P(\zeta)} d \zeta+b \mid P, R \in \mathbb{C}[z], \operatorname{deg} R=r, \operatorname{deg} P=d\right\},
$$

with $r=0$, that is, $\Psi_{X}(z)=\int_{z_{0}}^{z} \lambda^{-1} \mathrm{e}^{-P(\zeta)} d \zeta$ with $P(z)$ a polynomial of degree $d$. Finally, assign to $\mathcal{R}_{X}$ a flat metric $\left(\mathcal{R}_{X}, \pi_{X, 2}^{*}\left(\frac{\partial}{\partial t}\right)\right)$ induced by $\pi_{X, 2}$. By Proposition 3.5, our sought after vector field is

$$
X(z)=\Psi_{X}^{*}\left(\frac{\partial}{\partial t}\right)(z)=\lambda \mathrm{e}^{P(z)} \frac{\partial}{\partial z} \in \mathcal{E}(d)
$$

as required.

\footnotetext{
${ }^{16}$ This construction need only be applied if at stage (b) of the construction, some of the vertices $v_{l}$ on a given horizontal level have their corresponding values $\left\{a_{\sigma_{l}}\right\}$ on the same trajectory of $\frac{\partial}{\partial t}$.
} 
Remark 8.22. Each semi-infinite helicoid (associated to the finite asymptotic values $\left.a_{\sigma}\right)$ provided with the flat metric $\pi_{X, 2}^{*}\left(\frac{\partial}{\partial t}\right)$ is biholomorphic to a half-logarithmic spiral associated to the asymptotic values $a_{\sigma}$ and $\infty$.

8.5.3. Third part of the proof: The equivalence relation on $d$-configuration trees. Let us summarize the parameters that define the $d$-configuration trees:

1) We have $d-1$ parameters $\left\{\tilde{\lambda}_{\sigma \rho}\right\} \subset \widetilde{\mathbb{C}^{*}}$.

2) The choice of starting vertex (1) $=\left(\infty_{1}, a_{1}\right), a_{1} \in \mathbb{C}_{t}$, to traverse the $d$-configuration tree, which in fact is a choice of an asymptotic value (together with its exponential tract). Hence we are also determining a horizontal level 0 in the $d$-skeleton.

3) The choice of the lower limit of integration $z_{0}$ for the distinguished parameter $\Psi(z)=\int_{z_{0}}^{z} \omega_{X}$. Note that this choice is equivalent to choosing an "origin" $b_{0}=\left(z_{0}, 0\right) \in \mathcal{R}_{X}$.

Even though (2) and (3) above are related, they are not equivalent.

From the above, Remarks 8.2 and 8.18 , it is clear that there is a hidden equivalence relation amongst the $d$-configuration trees. In fact, given a $d$-configuration tree there are many choices for the starting vertex for the construction of $\mathcal{R}_{X}$ and for how to traverse the $d$-configuration tree. Moreover, recalling that there is a choice of the edge that needs to be removed when a horizontal cycle of diagonals appears on a horizontal subtree (see $88.5 .12 . \mathrm{b}$ ), it is clear that the $d$-skeletons obtained from any of the above choices are equivalent in the sense that they give rise to the same surface $\mathcal{R}_{X}$.

So we have:

Definition 8.23. Two $d$-configuration trees are equivalent,

$$
\Lambda_{1} \sim \Lambda_{2},
$$

if their corresponding $d$-skeletons are the same up to:

1. Relabelling of the vertices.

2. Choice of the horizontal level 0 .

3. Choice of which edge to remove when a horizontal cycle occurs (see 8.5.12.b where the edges of $\Lambda_{X}$ are assigned).

Note that the $d+1$ complex parameters, $\left\{z_{0},\left(\infty_{1}, a_{1}\right),\left\{\tilde{\lambda}_{\sigma \rho}\right\}_{1}^{d-1}\right\}$ defining $\Lambda_{X}$ are continuous, and they form local charts for $\mathcal{E}(d)$ as a complex manifold of dimension $d+1$.

The proof of Theorem 8.16 is done.

We now have three compatible atlases for $\mathcal{E}(d)$ as a complex manifold:

- The one given by $\left\{z_{0},\left(\infty_{1}, a_{1}\right),\left\{\tilde{\lambda}_{\sigma \rho}\right\}_{1}^{d-1}\right\}$ defining $\Lambda_{X}$.

- The more "natural" charts that arise from the $d+1$ coefficients $\left\{\left(b_{0}, \ldots, b_{d}\right)\right\} \subset$ $\mathbb{C}^{d+1}$ of the polynomial $P(z)$ defining $X \in \mathcal{E}(d)$.

- The final one given by the $d$ roots of $P(z)$ and the coefficient $\lambda$, as in Definition 8.1 .

8.6. Normal forms for $X \in \mathcal{E}(d)$, when $d=1,2,3$. We examine low degree cases for $X \in \mathcal{E}(d)$, where one can actually come up with explicit expressions for $\pi_{1}: \mathcal{E}(d) \rightarrow \mathcal{E}(d) / A u t(\mathbb{C})$ in diagram (1.2) and for the finite asymptotic values 
$\left\{a_{\sigma}\right\}$. A normal form is a representative of an equivalence class in $\mathcal{E}(d) / A u t(\mathbb{C})$, in general it is not possible to choose a canonical representative.

Theorem 8.24. The normal forms in $\mathcal{E}(d) / A u t(\mathbb{C}), d \leq 3$, can be given as follows:

For $d=1$,

$$
e^{z} \frac{\partial}{\partial z}
$$

For $d=2, \quad \mu e^{z^{2}} \frac{\partial}{\partial z}, \quad \mu \in \mathbb{C}^{*}$.

For $d=3, \quad \mu e^{\left(-\frac{1}{3} z^{3}+p z\right)} \frac{\partial}{\partial z}, \quad \mu \in \mathbb{C}^{*}, p \in \mathbb{C}$.

Furthermore, explicit expressions for the finite asymptotic values are given by (8.19), (8.21), (8.28), for $d=1,2,3$, respectively.

Proof.

Case $d=1$ (Normal form). Given a singular analytic vector field

$$
X(z)=\mathrm{e}^{\left(b_{0} z+b_{1}\right)} \frac{\partial}{\partial z}, \quad b_{0} \neq 0,
$$

then by considering the affine transformation $T(w)=\frac{1}{b_{0}} w-\frac{b_{1}+\log b_{0}}{b_{0}}$, the pullback vector field is

$$
\tilde{X}(w)=\left(T^{*} X\right)(w)=\mathrm{e}^{w} \frac{\partial}{\partial w} .
$$

Finite asymptotic value. If $\Psi(z)=\int_{z_{0}}^{z} \mathrm{e}^{-b_{0} \zeta-b_{1}} d \zeta$, then a straightforward calculation shows that

$$
\Psi(z)=b_{0}^{-1}\left(\mathrm{e}^{-\left(b_{0} z_{0}+b_{1}\right)}-\mathrm{e}^{-\left(b_{0} z+b_{1}\right)}\right),
$$

hence letting $b_{0} z=r \mathrm{e}^{i \theta}$ one has that

$$
\lim _{r \rightarrow \infty} \mathrm{e}^{-\left(r \mathrm{e}^{i \theta}+b_{1}\right)}= \begin{cases}0 & \theta \in\left(-\frac{\pi}{2}, \frac{\pi}{2}\right), \\ \infty & \theta \in\left(\frac{\pi}{2}, \frac{3 \pi}{2}\right), \\ \text { DNE } & \theta=\frac{\pi}{2}, \frac{3 \pi}{2} .\end{cases}
$$

So the finite asymptotic value of $\Psi(z)$ is

$$
b_{0}{ }^{-1} \mathrm{e}^{-\left(b_{0} z_{0}+b_{1}\right)},
$$

with finite determination paths being those paths $\gamma$ that tend to $\infty \in \widehat{\mathbb{C}}_{z}$ with argument in $\left(-\frac{\pi}{2}-\arg \left(b_{0}\right), \frac{\pi}{2}-\arg \left(b_{0}\right)\right)$. See Example 8.12,1 and Figure 11, a for the 1-configuration tree $\Lambda$ and its soul.

Case $d=2$ (Normal form). Given a singular analytic vector field of the form

$$
X(z)=\mathrm{e}^{\left(b_{0} z^{2}+b_{1} z+b_{2}\right)} \frac{\partial}{\partial z}, \quad b_{0} \neq 0,
$$

then by considering the affine transformations $T_{j}(w)=\frac{1}{\sqrt{b_{0}}} w-\frac{b_{1}}{2 b_{0}}$, where $j=1,2$ indicates the choice of square root, the pullback vector field is

$$
\tilde{X}(w)=\left(T_{j}^{*} X\right)(w)=\mu_{j} \mathrm{e}^{w^{2}} \frac{\partial}{\partial w}, \text { with } \mu_{j}=\sqrt{b_{0}} \exp \left(-\frac{b_{1}^{2}}{4 b_{0}}+b_{2}\right) .
$$

Hence $\mu_{j} \in \mathbb{C}^{*}$ is the complex parameter that completely determines the class of $X \in \mathcal{E}(2) / A u t(\mathbb{C})$. See Figures [15 and [16 for the generic examples of the phase portraits. 
Finite asymptotic values. If $\Psi(z)=\int_{z_{0}}^{z} \mathrm{e}^{-b_{0} \zeta^{2}-b_{1} \zeta-b_{2}} d \zeta$, then there are exactly two finite asymptotic values of $\Psi(z)$ given by 17

$$
\begin{aligned}
& a_{1}=\frac{\sqrt{\pi}}{2 \mu_{j}}\left(-\operatorname{erf}\left(\sqrt{b_{0}} z_{0}+\frac{b_{1}}{2 \sqrt{b_{0}}}\right)+1\right), \\
& a_{2}=\frac{\sqrt{\pi}}{2 \mu_{j}}\left(-\operatorname{erf}\left(\sqrt{b_{0}} z_{0}+\frac{b_{1}}{2 \sqrt{b_{0}}}\right)-1\right),
\end{aligned}
$$

with finite determination paths being those paths $\gamma$ that tend to $\infty$ with argument in $\left[-\frac{\pi}{4}-\arg \sqrt{b_{0}}, \frac{\pi}{4}-\arg \sqrt{b_{0}}\right]$ or $\left[\frac{3 \pi}{4}-\arg \sqrt{b_{0}}, \frac{5 \pi}{4}-\arg \sqrt{b_{0}}\right]$.

Let $\gamma_{z}$ be a path starting at $z_{0}$ and ending at $z$ in $\mathbb{C}$. From 8.20)

$$
\Psi(z)=\int_{z_{0}}^{z} \mathrm{e}^{-b_{0} \eta^{2}-b_{1} \eta-b_{2}} d \eta=\int_{T_{j}^{-1}\left(z_{0}\right)}^{T_{j}^{-1}(z)} \frac{1}{\mu_{j}} \mathrm{e}^{-\zeta^{2}} d \zeta,
$$

and letting $w_{0}=T_{j}^{-1}\left(z_{0}\right)$ and $w=T_{j}^{-1}(z)$, we have that

$$
\int_{w_{0}}^{w} \frac{1}{\mu_{j}} \mathrm{e}^{-\zeta^{2}} d \zeta=\left(\int_{w_{0}}^{0}+\int_{0}^{w}\right) \frac{1}{\mu_{j}} \mathrm{e}^{-\zeta^{2}} d \zeta
$$

Now noticing that

$$
\frac{1}{\mu_{j}} \int_{w_{0}}^{0} \mathrm{e}^{-\zeta^{2}} d \zeta=\frac{1}{\mu_{j}} \int_{0}^{-w_{0}} \mathrm{e}^{-\zeta^{2}} d \zeta=\frac{\sqrt{\pi}}{2 \mu_{j}} \operatorname{erf}\left(-w_{0}\right)=-\frac{\sqrt{\pi}}{2 \mu_{j}} \operatorname{erf}\left(w_{0}\right),
$$

and since

$$
\lim _{\substack{z \rightarrow \infty \\ z \in \gamma_{z}}} \operatorname{erf}\left(T_{j}^{-1}(z)\right)= \begin{cases}1 & \text { when } \arg \left(T_{j}^{-1}(z)\right) \in\left[-\frac{\pi}{4}, \frac{\pi}{4}\right], \\ -1 & \text { when } \arg \left(T_{j}^{-1}(z)\right) \in\left[\frac{3 \pi}{4}, \frac{5 \pi}{4}\right], \\ \infty & \text { when } \arg \left(T_{j}^{-1}(z)\right) \in\left(\frac{\pi}{4}, \frac{3 \pi}{4}\right) \cup\left(\frac{5 \pi}{4}, \frac{7 \pi}{4}\right),\end{cases}
$$

equation (8.21) follows. Note that the closed intervals where the limit is finite is not a typographical mistake, but a characteristic behaviour of the error function.

From Corollary 4.18, the configuration of finite asymptotic values modulo translations is the parameter that determines the class

$$
\left[\left[a_{1}, a_{2}\right]\right]= \pm\left(a_{2}-a_{1}\right)=\frac{\sqrt{\pi}}{\mu_{j}}=\lambda_{12}, \quad \mu_{j}= \pm \frac{\sqrt{\pi}}{a_{1}-a_{2}} .
$$

We have an orbifold structure for

$$
\frac{\mathcal{E}(2)}{\operatorname{Aut}(\mathbb{C})} \cong \frac{\mathbb{C}^{*}}{\mathbb{Z}_{2}}=\frac{\left\{\left[\left[a_{1}, a_{2}\right]\right]\right\}}{\mathbb{Z}_{2}},
$$

where the $\mathbb{Z}_{2}$ action identifies $\mu_{j}$ with $-\mu_{j}$. It is convenient to note that in this case the asymptotic values $a_{1}$ and $a_{2}$ always share a sheet on $\mathcal{R}_{X}$ and, in fact, the segment $\overline{b_{1} b_{2}} \in \pi_{X, 1}^{-1}\left(\overline{a_{1} a_{2}}\right)$ is the unique diagonal $\Delta_{12}$. See Example [8.12, 2 and Figure 11,b for the corresponding 2-configuration tree $\Lambda_{X}$ and its soul.

Case $d=3$ (Normal form). Given a singular analytic vector field of the form

$$
X(z)=\exp \left(b_{0} z^{3}+b_{1} z^{2}+b_{2} z+b_{3}\right) \frac{\partial}{\partial z}, \quad b_{0} \neq 0
$$

\footnotetext{
${ }^{17}$ Here erf is the usual error function originating from the integral of the exponential of $z^{2}$.
} 
there exists 3 affine transformations $T_{j}, j=1,2,3$, such that the pullback vector field is

$$
\widetilde{X}(w)=\left(T_{j}^{*} X\right)(w)=\mu \exp \left(-\frac{1}{3} w^{3}+p w\right) \frac{\partial}{\partial w} .
$$

In order to show this consider the affine transformations $T_{j}(w)=\alpha_{j} w+\beta_{j}$ with $\alpha_{j}=\left(-\frac{1}{3 b_{0}}\right)^{1 / 3}$ and $\beta_{j}=-\frac{b_{1}}{3 b_{0}}$, where $j=1,2,3$ indicates a choice of the cube root, then the pullback vector field is (8.24) with

$$
\mu=\left(-3 b_{0}\right)^{1 / 3} \exp \left(\frac{2 b_{1}^{3}-9 b_{0} b_{1} b_{2}+27 b_{0}^{2} b_{3}}{27 b_{0}^{2}}\right) \quad \text { and } \quad p=\frac{b_{1}^{2}-3 b_{0} b_{2}}{\left(-3 b_{0}\right)^{4 / 3}}
$$

where we omit the sub-index $j$ indicating the choice of cube root in $\mu$ and $p$.

Thus showing that in case $d=3$ the local geometry of the normal form is completely understood by the local parameters $\mu \neq 0$ and $p$. In fact, $\mathcal{E}(3) / \operatorname{Aut}(\mathbb{C})$ is a complex analytic variety of (complex) dimension 2 .

\section{Finite asymptotic values.}

Lemma 8.25. Let

$$
\mathcal{A} i(p, z)=\frac{1}{2 \pi i} \int_{\mathcal{L}(z)} e^{\frac{1}{3} \zeta^{3}-p \zeta} d \zeta
$$

be the Airy integral, where $A=\{z \in \mathbb{C} \mid \arg (z) \in(\pi / 6,3 \pi / 6)\}, \mathcal{L}(z):=\mathcal{L}(z, \tau)$ : $[0,1] \longrightarrow A$ is a simple $C^{1}$ path starting at 0 and ending at $z$, then:

1) $\mathcal{A} i(p, z)$ is an entire function of $p$ and is analytic in $A$ as a function of $z$.

2) Furthermore, $\mathcal{A} i(p, z)$ is in fact analytic in $\widehat{A}=A \cup e^{i 2 \pi / 3} A \cup e^{-i 2 \pi / 3} A$ and $\mathcal{A} i\left(p, e^{ \pm i 2 \pi / 3} z\right)=e^{ \pm i 2 \pi / 3} \mathcal{A} i\left(e^{ \pm i 2 \pi / 3} p, z\right)$.

Proof. Let $\mathcal{L}(z)$ be a path as above and let $\overline{\mathcal{L}(z)}$ be the conjugate path traversed in the opposite direction (i.e. starting at $\bar{z}$ and ending at 0 ). Then $\widehat{\mathcal{L}}(z)=\mathcal{L}(z) \cup \overline{\mathcal{L}(z)}$ is a piecewise $C^{1}$ simple path symmetrical about the real axis in $\mathbb{C}$ that starts at $\bar{z}$ and ends at $z$. Denote by $\widehat{\mathcal{L}}$ the limit path obtained from $\widehat{\mathcal{L}}(z)$ by letting $z$ tend to infinity along $A$. Then

$$
\operatorname{Ai}(p)=\frac{1}{2 \pi i} \int_{\widehat{\mathcal{L}}} \mathrm{e}^{\frac{1}{3} \zeta^{3}-p \zeta} d \zeta
$$

is the usual Airy integral which is in fact an entire function of $p$ (see [56], pp. 53). Moreover,

$$
\begin{aligned}
\operatorname{Ai}(p) & =\frac{1}{2 \pi i} \int_{\widehat{\mathcal{L}}} \mathrm{e}^{\frac{1}{3} \zeta^{3}-p \zeta} d \zeta \\
& =\lim _{\substack{z \rightarrow \infty \\
z \in A}}\left\{\frac{1}{2 \pi i} \int_{\mathcal{L}(z)} \mathrm{e}^{\frac{1}{3} \zeta^{3}-p \zeta} d \zeta+\frac{1}{2 \pi i} \int_{\overline{\mathcal{L}(z)}} \mathrm{e}^{\frac{1}{3} \zeta^{3}-p \zeta} d \zeta\right\} \\
& =\lim _{\substack{z \rightarrow \infty \\
z \in A}}\left\{\mathcal{A} i(p, z)+\frac{1}{2 \pi i} \int \frac{}{\mathcal{L}(z)} \mathrm{e}^{\frac{1}{3} \zeta^{3}-p \zeta} d \zeta\right\}
\end{aligned}
$$

hence in fact $\mathcal{A} i(p, z)$ is an entire function of $p$, and is analytic as a function of $z \in A$, as stated. 
For the proof of statement (2) of the lemma, consider that

$$
\begin{array}{rl}
\mathrm{e}^{ \pm i 2 \pi / 3} & \mathcal{A} i\left(\mathrm{e}^{ \pm i 2 \pi / 3} p, z\right) \\
& =\frac{e^{ \pm i 2 \pi / 3}}{2 \pi i} \int_{\mathcal{L}(z)} \mathrm{e}^{\frac{1}{3} \zeta^{3}-\left(\mathrm{e}^{ \pm i 2 \pi / 3} p\right) \zeta} d \zeta \\
& \left.=\frac{1}{2 \pi i} \int_{\mathcal{L}(z)} \mathrm{e}^{\frac{1}{3}\left(\mathrm{e}^{ \pm i 2 \pi / 3} \zeta\right)^{3}-p\left(\mathrm{e}^{ \pm i 2 \pi / 3} \zeta\right.}\right) \mathrm{e}^{ \pm i 2 \pi / 3} d \zeta \\
& =\frac{1}{2 \pi i} \int_{\mathrm{e}^{ \pm i 2 \pi / 3} \mathcal{L}(z)} \mathrm{e}^{\frac{1}{3} \zeta^{3}-p \zeta} d \zeta=\mathcal{A} i\left(p, \mathrm{e}^{ \pm i 2 \pi / 3} z\right) .
\end{array}
$$

Furthermore, note that the last integral appearing in (8.25) is in fact equal to

$$
\begin{array}{r}
\frac{1}{2 \pi i} \int_{\overline{\mathcal{L}(z)}} \mathrm{e}^{\frac{1}{3} \zeta^{3}-p \zeta} d \zeta=-\frac{1}{2 \pi i} \int_{0}^{\bar{z}} \mathrm{e}^{\frac{1}{3} \zeta^{3}-p \zeta} d \zeta=-\frac{1}{2 \pi i} \int_{0}^{e^{-i 2 \pi / 3} z} \mathrm{e}^{\frac{1}{3} \zeta^{3}-p \zeta} d \zeta \\
=-\mathcal{A} i\left(p, \mathrm{e}^{-i 2 \pi / 3} z\right)=-\mathrm{e}^{-i 2 \pi / 3} \mathcal{A} i\left(\mathrm{e}^{-i 2 \pi / 3} p, z\right),
\end{array}
$$

for $z \in A$, so in fact, we have

$$
\operatorname{Ai}(p)=\mathcal{A} i(p)-\mathrm{e}^{-i 2 \pi / 3} \mathcal{A} i\left(\mathrm{e}^{-i 2 \pi / 3} p\right), \quad \mathcal{A} i(p)=\lim _{\substack{z \rightarrow \infty \\ z \in A}} \mathcal{A} i(p, z) .
$$

Corollary 8.26. Let $\Psi(z)=\int_{w_{0}}^{w} \frac{1}{\mu} e^{\frac{1}{3} \zeta^{3}-p \zeta} d \zeta$, where $w=T_{j}^{-1}(z)$ and $w_{0}=$ $T_{j}^{-1}\left(z_{0}\right)$, then there are exactly 3 finite asymptotic values of $\Psi(z)$ given by

$$
a_{j+1}(\mu, p)=\frac{2 \pi i}{\mu}\left(\eta^{j} \mathcal{A} i\left(\eta^{j} p\right)-\mathcal{A} i\left(p, w_{0}\right)\right), j=0,1,2, \quad \eta=e^{i 2 \pi / 3},
$$

with finite determination paths ending in $A=\{z \in \mathbb{C} \mid \arg (z) \in(\pi / 6,3 \pi / 6)\}$, $e^{i 2 \pi / 3} A$ and $e^{-i 2 \pi / 3} A$ for $j=0,1$ and 2 , respectively.

Proof. It is a straightforward calculation using the previous lemma. The three finite asymptotic values depend on the specific finite determination path $\gamma$ chosen:

- if $\gamma$ ends in $A=\{z \in \mathbb{C} \mid \arg (z) \in(\pi / 6,3 \pi / 6)\}$, then $j=0$ so $\eta^{j}=1$,

- if $\gamma$ ends in $\mathrm{e}^{i 2 \pi / 3} A$, then $j=1$ so $\eta^{j}=\mathrm{e}^{i 2 \pi / 3}$,

- if $\gamma$ ends in $\mathrm{e}^{-i 2 \pi / 3} A$, then $j=2$ so $\eta^{j}=\mathrm{e}^{-i 2 \pi / 3}$.

In the case of $\mathcal{E}(3)$, the configuration of finite asymptotic values modulo translations (see once again Corollary 4.18) are the classes

$$
\left[\left[a_{1}, a_{2}, a_{3}\right]\right]
$$

consisting of the three finite asymptotic values modulo translations, which are in 1-1 correspondence with the triangles having vertices $a_{1}, a_{2}, a_{3}$. Using (8.27), it follows that the sides of the triangle $\left[\left[a_{1}, a_{2}, a_{3}\right]\right]$ are given by the absolute values of

$$
\begin{aligned}
& \widetilde{\lambda}_{13}=a_{3}-a_{1}=-\frac{2 \pi i}{\mu} \operatorname{Ai}(p), \\
& \widetilde{\lambda}_{21}=a_{1}-a_{2}=-\mathrm{e}^{i 2 \pi / 3} \frac{2 \pi i}{\mu} \operatorname{Ai}\left(\mathrm{e}^{i 2 \pi / 3} p\right), \\
& \widetilde{\lambda}_{32}=a_{2}-a_{3}=\frac{2 \pi i}{\mu}\left[\operatorname{Ai}(p)-\mathrm{e}^{i 2 \pi / 3} \operatorname{Ai}\left(\mathrm{e}^{i 2 \pi / 3} p\right)\right],
\end{aligned}
$$

where $\tilde{\lambda}_{j k}=\lambda_{j k} \mathrm{e}^{i 2 \pi K(j, k)}$, with $\lambda_{j k} \in \mathbb{C}^{*}$ and $K(j, k) \in \mathbb{Z}$, as in (8.11). As seen in the explicit construction of the vertices of the triangle (also see [53], pp. 291), it is not possible to have a fully degenerate triangle (all finite asymptotic values of $\Psi$ being the same). This finishes the proof of Theorem 8.24,

With the above conventions, we have proved the following. 
Corollary 8.27. The 3 -configuration tree $\Lambda_{X}$, for $X$ given by (8.24), is

$$
\Lambda_{X}=\left\{(1)=\left(\infty_{1}, a_{1}\right), 2=\left(\infty_{2}, a_{2}\right),(3)=\left(\infty_{3}, a_{3}\right) ;\left(e_{21}, \tilde{\lambda}_{21}\right),\left(e_{13}, \tilde{\lambda}_{13}\right)\right\},
$$

where we choose (2) to be the starting vertex, hence $\widetilde{\lambda}_{21} \in \mathbb{C}^{*}$.

Remark 8.28. This relates to Example 8.123 and Figure 11.

The 3-configuration tree and its soul in Figure 11. correspond to the case when the diagonals all share the same sheet $\mathbb{C} \backslash\left\{L_{a_{1}} \cup L_{a_{2}} \cup L_{a_{3}}\right\}$.

On the other hand, Figure 11, d corresponds to the case when the diagonal $\overline{(2)(1)}$ lies in the sheet $\mathbb{C} \backslash\left\{L_{a_{1}} \cup L_{a_{2}}\right\}$ and the diagonal (1)(3) lies in the sheet $\mathbb{C} \backslash\left\{L_{a_{1}} \cup L_{a_{3}}\right\}$ forming two horizontal subtrees separated by a vertical edge on the 3 -skeleton.

Note that $\infty_{1}, \infty_{2}, \infty_{3}$ corresponds to the exponential tract denoted by $A, \mathrm{e}^{i 2 \pi / 3} A$, $\mathrm{e}^{-i 2 \pi / 3} A$, respectively, in Corollary 8.26. Moreover, these 3 equi-angular sectors at $\infty \in \widehat{\mathbb{C}}_{z}$ have 3 other sectors interspersed where $\Psi$ has $\infty$ as asymptotic value, this in accordance with Remark 8.7

8.6.1. Global geometry of $\mathcal{E}(3) / A u t(\mathbb{C})$. Contrary to the case $d=2($ see $(\underline{8.23})$ ), for $d=3$ we need more than just the triangles $\left[\left[a_{1}, a_{2}, a_{3}\right]\right]$ to determine $X$. In what follows we examine numerically the holomorphic mono-parametric family

$$
\mathbb{C}_{p} \longrightarrow \mathcal{E}(3), \quad p \longmapsto X(z)=\mathrm{e}^{-\frac{1}{3} z^{3}+p z} \frac{\partial}{\partial z},
$$

where we have fixed $\mu=1$ in (8.24), since $\mu$ acts as a homothety of the triangle $\left[\left[a_{1}, a_{2}, a_{3}\right]\right]$ of asymptotic values.

For each $p \in \mathbb{C}_{p}$, in Figure [14, a, corresponds a triangle $\left[\left[a_{1}, a_{2}, a_{3}\right]\right]$ in Figure 14. $\mathrm{b}$, where without loss of generality we assume that $a_{1}=0$. With the above assumptions, and considering (8.29) together with the notation of Remark 8.28, we can now observe the following (a Mathematica CDF file, corresponding to Figure 14, is available as electronic supplemental material).

1. The blue curves in Figure 14, a indicate where $\mathfrak{R e}\left(a_{3}\right)=0$, so in the corresponding triangle the side $\overline{a_{1} a_{3}}$ (depicted red in Figure 14, b) is vertical; the red curves in Figure 14, a are where $\mathfrak{I m}\left(a_{3}\right)=0$, so the side $\overline{a_{1} a_{3}}$ is horizontal. The green and orange curves indicate where $\mathfrak{R e}\left(a_{2}\right)$ and $\mathfrak{I m}\left(a_{2}\right)$ are zero, respectively (the corresponding triangle will have the blue side $\overline{a_{2} a_{1}}$ being vertical or horizontal, respectively).

2. The blue dots correspond to the case of the degenerate triangle when $a_{3}=a_{1}=0$ (exactly on the zeros of the Airy function $\operatorname{Ai}(p)$ ). The black dots correspond to $a_{2}=a_{1}=0$, and the brown dots to $a_{3}=a_{2}$.

3. The green dots correspond to two particular choices of parameter $p$ that give rise to the two triangles depicted in Figure 14, b. The origin in $\mathbb{C}_{p}$ corresponds to the equilateral triangle depicted in Figure 14. b, while the other green dot (lying on the intersection of the dotted brown curve and one of the red curves) corresponds to the isosceles triangle.

4. In general for $p \in \mathbb{C}_{p}$ the segments (1)(3) $\overline{(2)(1)}$ and (2)(3) in $\mathcal{R}_{X}$, are not all diagonals (recall that $\pi_{X, 2}\left(b_{j}\right)=a_{j}$ ); in fact, the three segments are all diagonals if and only if $a_{1}, a_{2}, a_{3}$ are all distinct and share the same sheet on $\mathcal{R}_{X}$. Furthermore, since we have fixed $a_{1}=0 \in \mathbb{C}_{t}$ we may assume without loss of generality that the side $\overline{a_{2} a_{3}}$ (colored purple) is never a projection of a diagonal (except in the case just considered). See Definition 8.8, Figure 11, c-d. 
5. By moving $p$ along one of the dotted lines (or along the negative real axis) the associated triangle changes and eventually repeats itself (modulo scale). We shall examine in detail three scenarios by considering the triangle $\left[\left[a_{1}, a_{2}, a_{3}\right]\right]$ on $\mathbb{C}_{t}$, and the diagonals on $\mathcal{R}_{X}$.

a) Moving $p$ along the negative axis (away from the origin) makes the side $\overline{a_{2} a_{1}}$ (colored blue) go around the origin counterclockwise, thus on $\mathcal{R}_{X}$ the corresponding diagonal (2)(1) changes sheet every time $p$ crosses two orange curves.

b) Moving $p$ along the dotted black lines (away from the origin) makes the side $\overline{a_{1} a_{3}}$ (colored red) go around the origin clockwise, thus on $\mathcal{R}_{X}$ the corresponding diagonal (1)(3) changes sheet every time $p$ crosses two red curves.

c) Moving $p$ along the dotted brown lines (away from the origin) makes the side $\overline{a_{1} a_{3}}$ (colored red) go around the origin counterclockwise and the side $\overline{a_{2} a_{1}}$ (colored blue) go around the origin clockwise, thus on $\mathcal{R}_{X}$ the corresponding diagonal (1)(3) changes sheet every time $p$ crosses two red curves and the corresponding diagonal (2)(1) changes sheet every time $p$ crosses two orange curves.

6. Relating the configuration tree $\Lambda_{X}$ to (5) above, the argument of the weight $\lambda_{13}$ changes by $\pm \pi$ every time that $p$ crosses either an orange or a red curve.

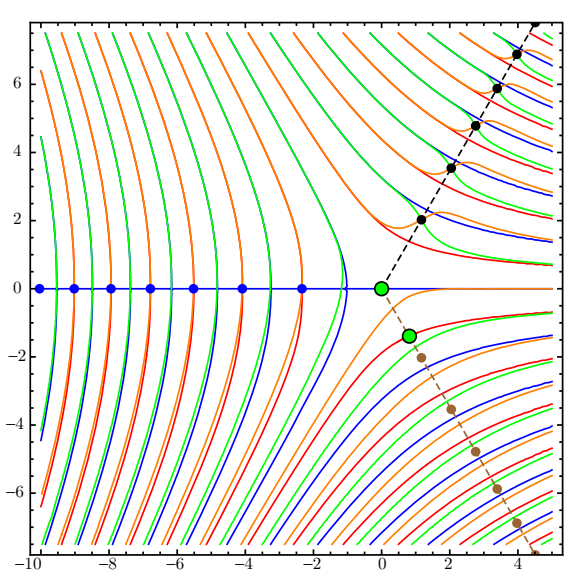

(a) $\mathbb{C}_{p}$ for fixed $\mu=1$.

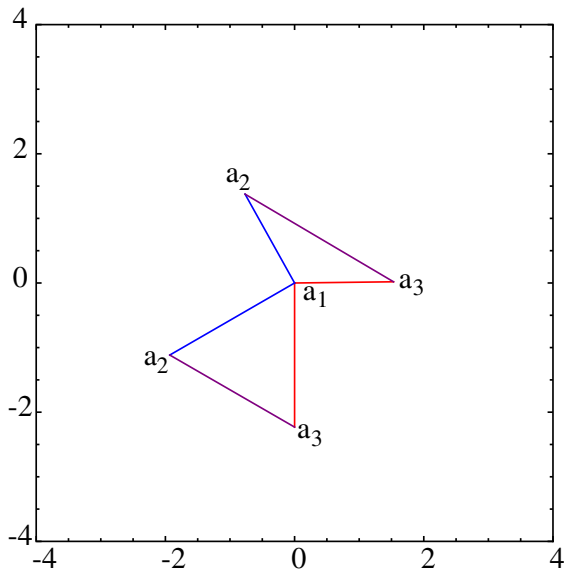

(b) Triangles $\left[\left[a_{1}, a_{2}, a_{3}\right]\right]$.

Figure 14. We depict in (a) the $p$-parameter plane $\mathbb{C}_{p}$ for $\mathcal{E}(3) /$ Aut $(\mathbb{C})$ fixing $\mu=1$, and (b) the corresponding triangles $\left[\left[a_{1}, a_{2}, a_{3}\right]\right]$, with $a_{1}=0$, in $\mathbb{C}_{t}$. The green dot at the origin in (a) corresponds to the equilateral triangle, the other green dot corresponds to the isosceles triangle. Moving the parameter $p$ in (a) deforms the corresponding triangle in (b). See 8.6 .1 for further details.

8.7. The quotient space $\mathcal{E}(d) / A u t(\mathbb{C})$ and flat metrics. Continuing with the study, started in 8.6 , of the normal forms $\mathcal{E}(d) / A u t(\mathbb{C})$ for general $d \geq 2$, and 
including the flat metrics $\mathcal{E}(d) /\left(\operatorname{Aut}(\mathbb{C}) \times S^{1}\right)$ alluded to in diagram (1.2), we can now state and prove the following result.

Corollary 8.29 (Normal forms and flat metrics up to isometry, $d \geq 2$ ).

1) Normal forms: $\frac{\mathcal{E}(d)}{\operatorname{Aut}(\mathbb{C})}$ is a complex manifold of dimension $d-1$.

2) Flat metrics: $\frac{\mathcal{E}(d)}{\operatorname{Aut}(\mathbb{C}) \times S^{1}}$ is a real analytic manifold of dimension $2 d-3$.

Note that the complex dimension alluded to in (1) of the corollary has been explicitly calculated, in the previous section, for the cases $d=1,2,3$.

Proof. The space of polynomials can be parametrized by the roots up to the action of the symmetric group of order $d, S(d)$, hence we get the diagram

$$
\begin{array}{ccccc}
\left(\mathbb{C}^{*}\right)^{2} \times\left(\frac{\left.\mathbb{C}_{\text {roots }}^{d}\right)}{\mathcal{S}(d)}\right) & \rightarrow & \mathbb{C}^{*} \times \mathbb{C}_{\text {coef }}^{d+1} & \rightarrow & \mathcal{E}(d) \\
\left(\lambda, b_{0},\left[p_{1}, \ldots, p_{d}\right]\right) & \mapsto & \left(\lambda, b_{0}, \ldots, b_{d}\right) & \mapsto & \lambda \exp \left(b_{0} z^{d}+\ldots+b_{d}\right) \frac{\partial}{\partial z},
\end{array}
$$

here $\left[p_{1}, \ldots, p_{d}\right]$ means the unordered roots and

$$
\left(z-p_{1}\right) \cdots\left(z-p_{d}\right)=z^{d}+\left(b_{1} / b_{0}\right) z^{d-1}+\ldots+\left(b_{d} / b_{0}\right) .
$$

A virtue of the parametrization using the roots (8.30) and the redundant parameter $\lambda$, is as follows. The action of the affine group $\operatorname{Aut}(\mathbb{C})=\{w \mapsto \mathrm{a} w+\mathrm{b}=z\}$ by pullback is

$$
\begin{array}{ccc}
\operatorname{Aut}(\mathbb{C}) \times \mathcal{E}(d) & \longrightarrow & \mathcal{E}(d) \\
\left(\mathrm{a} w+\mathrm{b},\left(\lambda, b_{0},\left[p_{1}, \ldots, p_{d}\right]\right)\right) & \longmapsto & \left(\lambda \mathrm{a}^{-1}, b_{0} \mathrm{a}^{d},\left[T^{-1}\left(p_{1}\right), \ldots, T^{-1}\left(p_{d}\right)\right]\right) .
\end{array}
$$

In fact, given $\left(\lambda, b_{0},\left[p_{1}, \ldots, p_{d}\right]\right)$ and $T(w)=\mathrm{a} w+\mathrm{b}=z$, we can compute

$$
\begin{gathered}
T^{*}\left(\lambda \exp \left(b_{0}\left(z-p_{1}\right) \cdots\left(z-p_{d}\right)\right) \frac{\partial}{\partial z}\right)=\lambda \exp \left(b_{0}\left(\mathrm{a} w+\mathrm{b}-p_{1}\right) \cdots\left(\mathrm{a} w+\mathrm{b}-p_{d}\right)\right) \frac{\partial}{\partial z} \\
=\frac{\lambda}{\mathrm{a}} \exp \left(b_{0} \mathrm{a}^{d}\left(w-T^{-1}\left(p_{1}\right)\right) \cdots\left(w-T^{-1}\left(p_{d}\right)\right)\right) \frac{\partial}{\partial w}
\end{gathered}
$$

Clearly, for $d \geq 2$ this is a proper action and its isotropy group is trivial, so it is free. Considering also the action of $S^{1}$ by rotations $X \mapsto \mathrm{e}^{i \theta} X$, it is clear that this is also a proper and free action.

As is usual the triviality of the isotropy group of $X \in \mathcal{E}(d)$ has geometric implications on the quotient spaces. Following [20], pp. 53: the action of $A u t(\mathbb{C})$ on $\mathcal{E}(d)$ is proper with isotropy group the identity, hence the quotient space is a manifold of dimension $\operatorname{dim}(\mathcal{E}(d))-\operatorname{dim}(\operatorname{Aut}(\mathbb{C}))$. The analogous fact holds for the action of $\operatorname{Aut}(\mathbb{C}) \times S^{1}$.

8.8. On the cardinality of the topological classes of $\mathfrak{R e}(X)$, for $X \in \mathcal{E}(d)$. The previous section shows that the analytical and metric classification are very fine. In order to have a coarser classification, we will consider the notion (see Definition 3.1.1) of topological equivalence of singular analytic vector fields under the action of

$$
\text { Homeo }(\mathbb{C})^{+}=\left\{h: \widehat{\mathbb{C}}_{z} \rightarrow \widehat{\mathbb{C}}_{z} \text { | orientation preserving homeomorphism fixing } \infty\right\} .
$$

In particular, we will be considering $\mathcal{E}(d) / \mathrm{Homeo}(\mathbb{C})^{+}$, i.e. the topological classes of phase portraits of $\mathfrak{R e}(X)$, for $X \in \mathcal{E}(d)$.

Recalling Definition 8.3 of a sheet of $\mathcal{R}_{X}$, Definition 8.8 of a diagonal associated to a pair of asymptotic values and the description of $d$-configuration trees, we now 
present a first step towards the topological classification of $\mathfrak{R e}(X)$, for $X \in \mathcal{E}(d)$, identifying the canonical regions 18 in a sheet, hence the bifurcation mechanisms.

Remark 8.30 (Dynamical aspects of a sheet of $\mathcal{R}_{X}$, for $X \in \mathcal{E}(d)$ ).

Case 1. A sheet $\mathbb{C}_{t} \backslash\left\{L_{\mathrm{k}}\right\}$ with only one branch cut $L_{\mathrm{k}}$, determines two half-planes $\left(\overline{\mathbb{H}}_{ \pm}^{2}, \frac{\partial}{\partial t}\right)$ in the phase portrait of $\mathfrak{R e}(X)$ on $\mathbb{C}_{z}$.

Case 2. A sheet $\mathbb{C}_{t} \backslash\left\{L_{\mathrm{k}}\right\}_{\mathrm{k}=1}^{\mathrm{r}}$ having $r$ branch cuts with different heights (recall Definition 8.3) $\mathfrak{I m}\left(a_{1}\right)<\cdots<\mathfrak{I m}\left(a_{\mathrm{r}}\right)$ determines two half-planes $\left(\overline{\mathbb{H}}_{ \pm}^{2}, \frac{\partial}{\partial t}\right)$ and $r-1$ finite height strips flow $\left(\left\{\mathfrak{I m}\left(a_{\mathrm{k}}\right)<\mathfrak{I m}(z)<\mathfrak{I m}\left(a_{\mathrm{k}+1}\right)\right\}, \frac{\partial}{\partial t}\right)$ in the phase portrait of $\mathfrak{R e}(X)$ on $\mathbb{C}_{z}$.

Case 3. A sheet $\mathbb{C}_{t} \backslash\left\{L_{\mathrm{k}}\right\}_{\mathrm{k}=1,2}$ having two branch cuts in the same horizontal trajectory, say $(\infty, a] \cup\left[a+\tau_{0}, \infty\right)$ for $\tau_{0} \in \mathbb{R}^{+}$, determines two half-planes $\left(\mathbb{H}^{2}, \frac{\partial}{\partial t}\right)$, and one single finite homoclinic trajectory of $\mathfrak{R e}(X)$, thus isometric to a closed interval $\left[a, a+\tau_{0}\right]$ in the phase portrait. Recall that homoclinic means it is a trajectory which joins the singularity $\infty$ to itself in the phase portrait of $\mathfrak{R e}(X)$ on $\widehat{\mathbb{C}_{z}}$.

Case 4. Is the mixing of cases 2 and 3. If the sheet $\mathbb{C}_{t} \backslash\left\{L_{\mathrm{k}}\right\}_{\mathrm{k}=1}^{r}$ has (horizontal) branch cuts with at least two different imaginary values then at least one finite height strip flow is determined; moreover, if there are two or more branch cuts in the same horizontal trajectory these determine homoclinic trajectories. Of course the branch cuts with the smallest and the biggest heights determine two halfplanes. Whence this type of sheet determines half-planes, finite height strip flows and homoclinic trajectories for the phase portrait of $\mathfrak{R e}(X)$ on $\widehat{\mathbb{C}_{z}}$.

Theorem 8.31 (Cardinality of the topological classes $\mathcal{E}(d) /$ Homeo $(\mathbb{C})^{+}$).

For $d=1,2$, there are $d$ topological classes of $\mathfrak{R e}(X)$ for $X \in \mathcal{E}(d)$.

For each $d \geq 3$, there are infinite (numerable) topological classes of $\mathfrak{R e}(X)$ for $X \in \mathcal{E}(d)$.

Proof. Case $d=1$. Follows directly from Theorem 8.24,

Case $d=2$. We shall show that there are two distinct topologies for the real vector fields. Start by noticing that $X$ will have two distinct finite asymptotic values $a_{1}$ and $a_{2}$ whose corresponding diagonal $\Delta_{12}$ (associated to $\lambda_{12}$ in $\Lambda_{X}$ ) lies on the same sheet in $\mathcal{R}_{X}$. We now have two cases:

1) The diagonal $\Delta_{12}$ is not horizontal, we apply Remark 8.30, case 2 . That is, if $\mathfrak{I m}\left(a_{1}\right) \neq \mathfrak{I m}\left(a_{2}\right)$, then $\mathfrak{R e}(X)$ has a horizontal finite height strip flow

$$
S=\pi_{X, 1}\left(\left\{(z, t) \in \mathbb{C}_{\Delta_{12}} \subset \mathcal{R}_{X} \mid \mathfrak{I m}\left(a_{1}\right)<\mathfrak{I m}(t)<\mathfrak{I m}\left(a_{2}\right)\right\}\right),
$$

of height $\left|\mathfrak{I m}\left(a_{2}\right)-\mathfrak{I m}\left(a_{1}\right)\right|$, foliated by trajectories of $\mathfrak{R e}(X)$, or the analogous case when $\mathfrak{I m}\left(a_{2}\right)<\mathfrak{I m}(t)<\mathfrak{I m}\left(a_{1}\right)$; where $\mathbb{C}_{\Delta_{12}}$ denotes the sheet in $\mathcal{R}_{X}$ where the diagonal $\Delta_{12}$ lies (see the last paragraph of Definition 8.8).

2) The diagonal $\Delta_{12}$ is horizontal, we apply Remark 8.30, case 3 , hence a finite homoclinic trajectory.

\footnotetext{
${ }^{18}$ This notation for the phase portrait of $\mathfrak{R e}(X)$ on $\mathbb{C}_{z}$ follows the classical concepts of L. Markus; see [47] and Table 3 in 11 Appendix.
} 
Example 8.32 (Two phase portraits for $\left.\left\{\mathfrak{R e}\left(\lambda \mathrm{e}^{z^{2}} \frac{\partial}{\partial z}\right) \mid \lambda \in \mathbb{C}^{*}\right\}\right)$. Figure 15 shows the finite height strip flow in the phase portrait, generated by the nonhorizontal diagonal. Figure [16] shows the case when the diagonal is horizontal, thus degenerating the strip flow to a finite homoclinic trajectory.

Case $d \geq 3$. When $d \geq 3$, recall that by Theorem 8.16 there is a correspondence between $X \in \mathcal{E}(d)$ and $d$-configuration trees (and therefore its corresponding $d-$ skeleton), clearly the four cases in Remark 8.30 can appear. Moreover, from the description of the $d$-skeleton, notice that for $d \geq 3$ there will be at least 2, and generically $d-1$ different horizontal subtrees. On the other hand, horizontal subtrees are vertically separated from other horizontal subtrees by an integer number $|K(\sigma, \rho)|$ of vertical edges of the $d$-skeleton. By changing the integer $K(\sigma, \rho)$ we change the topology of $\mathfrak{R e}(X)$ since we are adding (or eliminating) $2 K(\sigma, \rho)$ halfplanes to (from) $\mathcal{R}_{X}$.

Example 8.33. 1. $d=3$ Numerical argument. Consider 88.6.1,6, Figure 14, By allowing $p \in \mathbb{C}_{p}$ to move, every time time $p$ crosses two consecutive orange curves or two consecutive red curves, $K(1,3)$ changes by an integer.

2. $d=3$ Combinatorial argument. This case can be appreciated in Figure 11.c where $K(1,3)=0$, and Figure 11, d where $K(1,3)=1$; in particular by adding copies of $\mathbb{C}_{t} \backslash\left\{L_{k}\right\}$ in between the sheets that contain the diagonals (2)(1) and (1)(3) one obtains an infinite number of different surfaces $\mathcal{R}_{X}$, and hence an infinite number of different topologies for $\mathfrak{R e}(X)$.

The interested reader can compare $\sqrt[8]{8}$ in the case $d=3$, with [53], pp. 292.

\section{From Germs to ADMisSible WORDS AND BACK}

In this section we shall explore the relationship between germs with an isolated essential singularity, that satisfy certain conditions on the logarithmic branch points and admissible words that have a specific number of sectors of type $\mathscr{E}$.

\subsection{Admissible words from germs $((\mathbb{C}, 0), X)$.}

Theorem 9.1 (Recognition of admissible words from germs). Let $((\mathbb{C}, 0), X)$ be a singular analytic vector field germ such that

1) 0 is an isolated essential singularity of $X$, and

2) the inverse $\Psi_{X}^{-1}$ of $\Psi_{X}:(\mathbb{C}, 0) \rightarrow \widehat{\mathbb{C}}_{t}$ has as unique singularities $d \geq 2$ logarithmic branch points over $d$ finite asymptotic values $\left\{a_{j}\right\} \subset \mathbb{C}_{t}$ and $d$ logarithmic branch points over $\infty \in \widehat{\mathbb{C}}_{t}$.

If $\gamma:[0,2 \pi] \rightarrow(\mathbb{C}, 0)$ is a simple path enclosing 0 , then there exists a deformation of $\gamma$, which determines an admissible word $\mathcal{W}_{X}$ at 0 , composed of sectors of type $H, E, P$ and $\mathscr{E}$. In particular, there are $\epsilon=2 d$ sectors of type $\mathscr{E}$.

Recall that the definitions in $\$ 4.3$, of asymptotic values, transcendental singularities, and logarithmic branch points make sense for an isolated essential singularity $e \in M$ or $(\mathbb{C}, 0)$ of $\Psi_{X}$. Moreover, the Definition 8.8 of diagonal also makes sense by replacing $\infty_{\sigma}, \infty_{\rho} \in \overline{\mathbb{C}}_{z}$ with the appropriate $e_{\sigma}, e_{\rho} \in \bar{M}$, where $\bar{M}$ is a suitable non-Hausdorff closure of $M \backslash\{e\}$ or ( $\mathbb{C}, 0) \backslash\{0\}$; see Remark 8.6.

To illustrate the difficulties and their solution, we consider the following. 

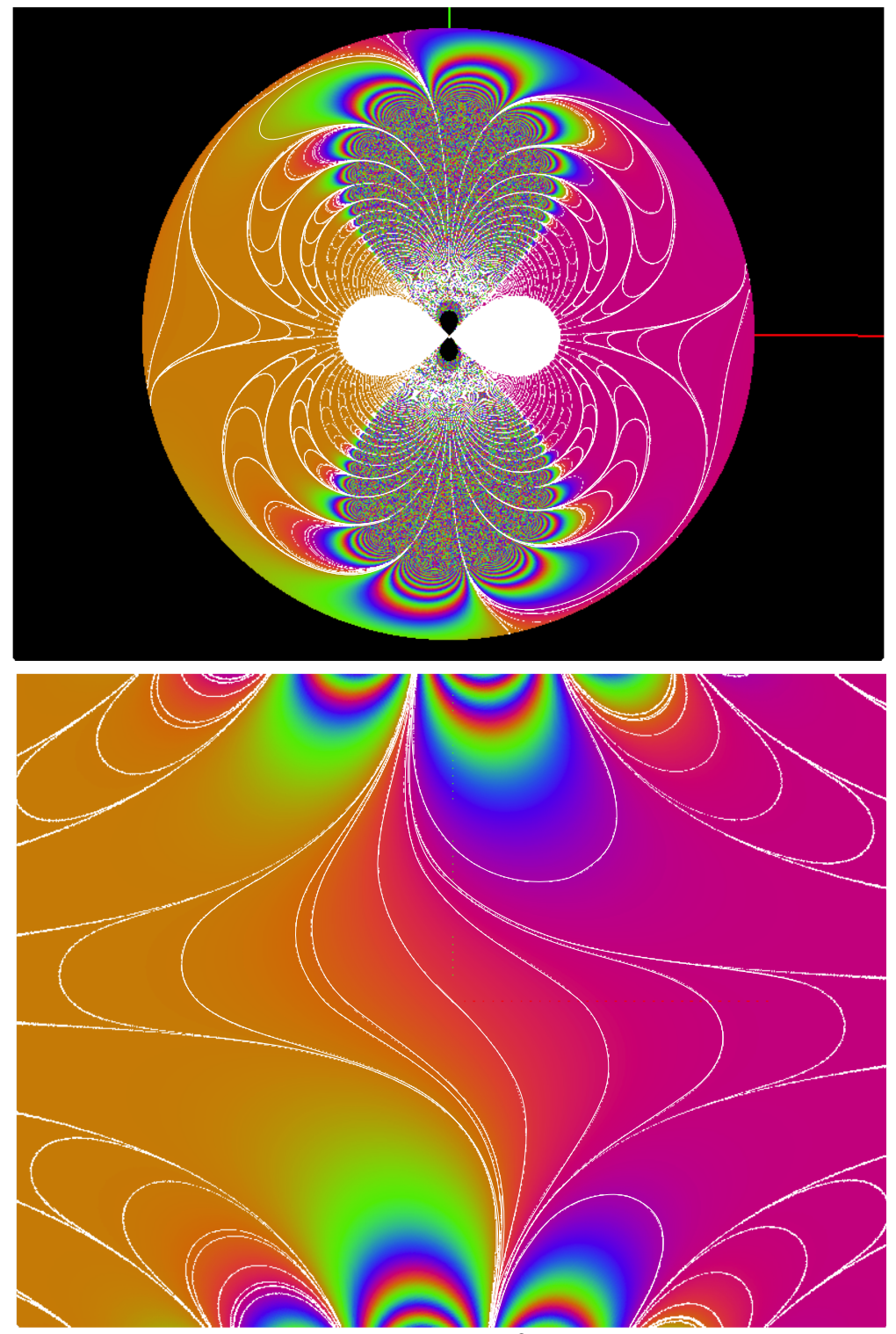

$$
X(z)=\mathrm{e}^{i \frac{\pi}{4}} \mathrm{e}^{z^{2}} \frac{\partial}{\partial z}
$$

Figure 15. One of the two different topologies for the phase portrait of $\mathfrak{R e}(X)$, when $d=2$. Note the appearance of a finite height strip flow in this figure, while in Figure 16] this degenerates to a finite homoclinic trajectory. This bifurcation of the topology of $\mathfrak{R e}(X)$ occurs when the diagonal $\Delta_{12} \subset \mathcal{R}_{X}$ is horizontal (i.e. $\lambda_{12}$ is strictly real). See Theorem 8.31 . 

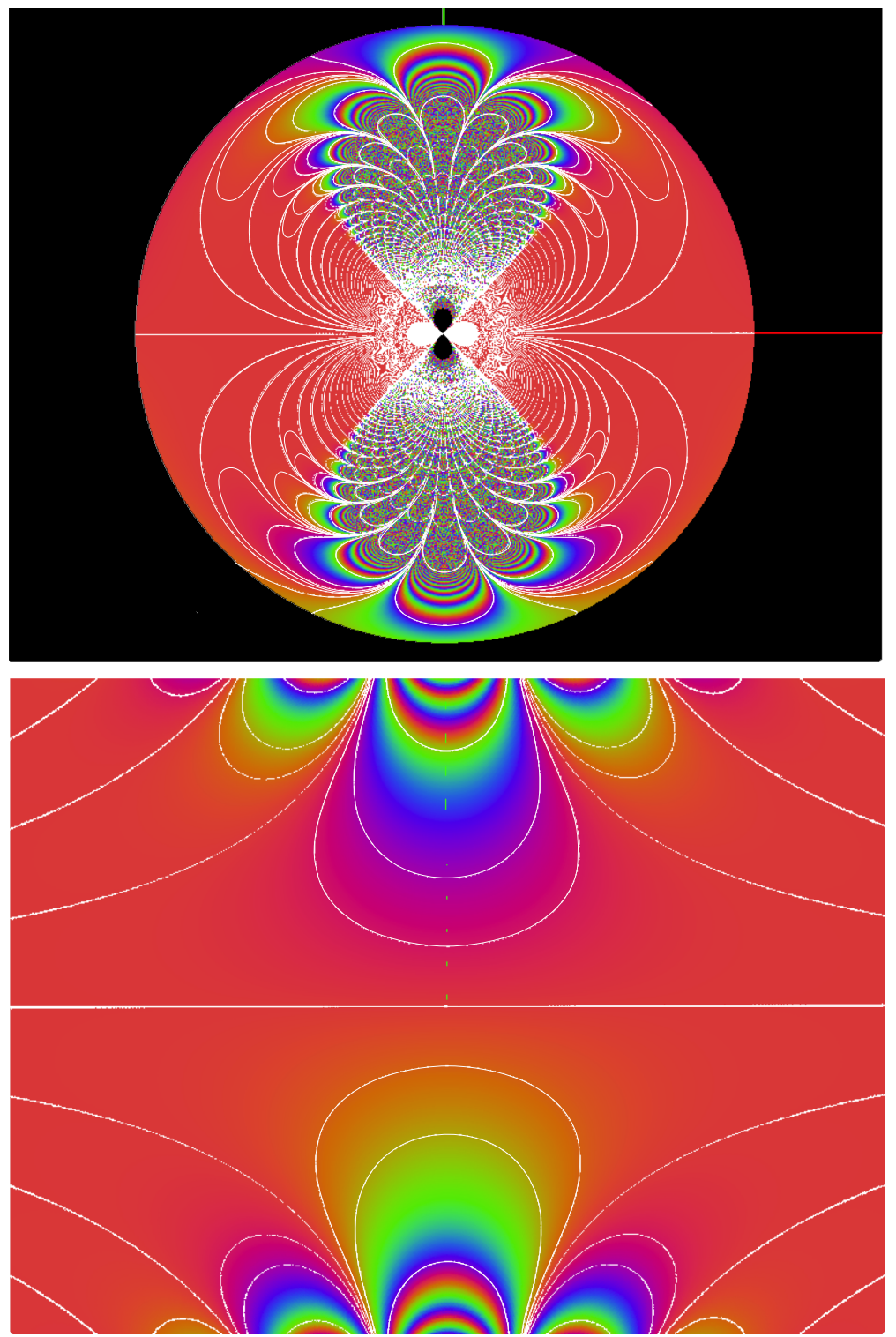

$$
X(z)=\mathrm{e}^{z^{2}} \frac{\partial}{\partial z}
$$

Figure 16. One of the two different topologies for the phase portrait of $\mathfrak{R e}(X)$, when $d=2$. In this figure the finite height strip flow, pictured in Figure 15, degenerates to a finite homoclinic trajectory. This bifurcation of the topology of $\mathfrak{R e}(X)$ occurs when the diagonal $\Delta_{12} \subset \mathcal{R}_{X}$ is horizontal (i.e. $\lambda_{12}$ is strictly real). See Theorem 8.31 
Example 9.2. Let $\left((\widehat{\mathbb{C}}, \infty), \mathrm{e}^{z} \frac{\partial}{\partial z}\right)$ be the germ, provided with $\gamma$ a simple path enclosing $\infty \in \widehat{\mathbb{C}}$. There is only one finite asymptotic value, say $a_{1}$. We assume that $\gamma$ is such that $\widetilde{\Gamma}(\tau):=(\gamma(\tau), \Gamma(\tau))$, for $\Gamma(\tau)=\left(\Psi_{X} \circ \gamma\right)(\tau)$, is contained in a copy of an upper half-plane $\overline{\mathbb{H}}_{+}^{2} \subset \mathcal{R}_{X}$. Recalling \$5.3.1 $X$ determines two half-logarithmic spirals. The soul of the Riemann surface $\mathcal{R}_{X} \backslash\{$ two half-logarithmic spirals $\}$, obtained from the graph of $\Psi_{X}$ over $\bar{U} \backslash\{\infty\}$, is the gray shaded region in Figure [7] a, having as boundary, the path $\widetilde{\Gamma}$ and two horizontal branch cuts $\left[a_{1}, \infty\right)_{+} \cup\left[a_{1}, \infty\right)_{-}$ similarly as in (8.2) and (8.3).

The original path $\gamma$, or $\widetilde{\Gamma}$, does not define a cyclic word for the germ of $X$.

However, there is a deformation of $\gamma$ towards its interior in $\mathcal{R}_{X}$ such that the respective deformation of $\widetilde{\Gamma}$ bounces off (recall Definition 5.3) the boundary of the half-logarithmic spiral of $\mathcal{R}_{X}$ determined by $\left[a_{1}, \infty\right)_{+}$. The deformed path is illustrated in Figure 17. b, it determines an entire sector flanked by a hyperbolic sector and an elliptic sector, by abuse of notation $H \mathscr{E} E$.

Figure 17. c shows a taut deformation $\widetilde{\Gamma}_{1} \cup \widetilde{\Gamma}_{2}$, where the path bounces off the halflogarithmic spirals associated to $\left[a_{1}, \infty\right)_{-}$and $\left[a_{1}, \infty\right)_{+}$. In turn this determines a word

$$
\underbrace{E \mathscr{E} H}_{\widetilde{\Gamma}_{1}} \underbrace{H \mathscr{E} E}_{\widetilde{\Gamma}_{2}}
$$

for the germ $X$. From $\widetilde{\Gamma}_{1} \cup \widetilde{\Gamma}_{2}$, we obtain the desired deformation of $\gamma$.

Proof. Step 1. For simplicity, let us start off by assuming that:

i) the asymptotic values $\left\{a_{j}\right\}_{j=1}^{d}$ lie on $d$ different horizontal trajectories of $\left(\mathbb{C}, \frac{\partial}{\partial t}\right)$, ii) $\operatorname{Res}\left(\omega_{X}, 0\right)=0$.

Let $\bar{U} \subset\left(\mathbb{C}_{z}, 0\right)$ be the closed region, whose boundary is a path $\gamma=\gamma(\tau)$, as in the theorem, and let $X$ be a germ representative. The associated Riemann surface $\mathcal{R}_{X} \subset \overline{\mathbb{C}}_{z} \times \widehat{\mathbb{C}}_{t}$ is determined by the graph of $\Psi_{X}$ over $\bar{U} \backslash\{0\}$.

$\mathcal{R}_{X}$ is connected, non compact and contains the path

$$
\widetilde{\Gamma}(\tau)=(\gamma(\tau), \Gamma(\tau)), \Gamma(\tau)=\left(\Psi_{X} \circ \gamma\right)(\tau) .
$$

To each deformation of $\gamma(\tau)$ in $\left(\mathbb{C}_{z}, 0\right)$ corresponds a deformation of $\widetilde{\Gamma}(\tau)$ in $\mathcal{R}_{X}$, and vice versa, since $\bar{U} \backslash\{0\}$ is biholomorphic to $\mathcal{R}_{X}$, similarly as in Remark 8.2 ,

The fundamental group of $\mathcal{R}_{X}$ is $\mathbb{Z}$, whose generator is $\widetilde{\Gamma}$.

From hypothesis (2), the boundary of $\mathcal{R}_{X} \subset \overline{\mathbb{C}}_{z} \times \widehat{\mathbb{C}}_{t}$ is exactly

$$
\widetilde{\Gamma} \cup\left\{\left(\infty_{j}, a_{j}\right)\right\}_{j=1}^{d} \cup\left\{\left(\infty_{j}, \infty\right)\right\}_{j=d+1}^{2 d},
$$

following the enumeration convened in Remarks 8.6 and 8.7 (a priori $X$ does not belong to $\mathcal{E}(d))$.

As a consequence, the analytic extension of $\Psi^{-1}$ along paths $\beta(\tau):[0, \varepsilon) \longrightarrow \mathbb{C}_{t}$ which avoid $\left\{a_{j}\right\}_{j=1}^{d}$ is possible. The boundary of $\mathcal{R}_{X}$ projects under $\pi_{X, 2}$ to $\Gamma \cup\left\{a_{j}\right\}_{j=1}^{d} \subset \mathbb{C}_{t}$.

Let $((\mathbb{C}, 0), X)$ be a singular analytic vector field germ, as in the theorem, the soul of the Riemann surface $\mathcal{R}_{X}$ is a connected surface with open interior

$$
\mathcal{R}_{X} \backslash\{\text { the } 2 d \text { half-logarithmic spirals of } X\} \text {. }
$$

For example, in Figure 17] a and Figure 19, the shaded regions determine the soul of $\mathcal{R}_{X}$, respectively. 
a)

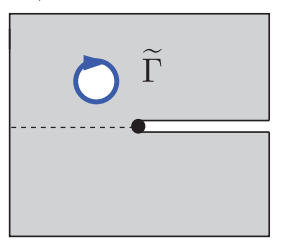

b)

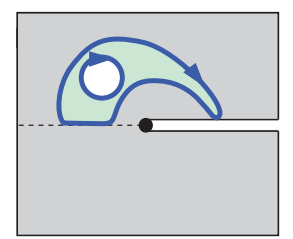

c)

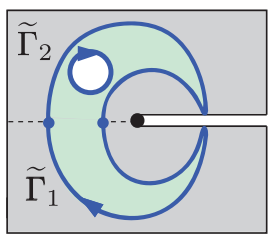

d)

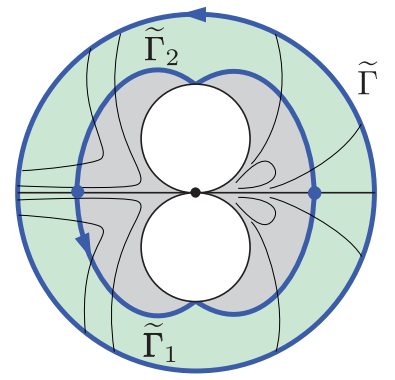

FIGURE 17. For $X(z)=\mathrm{e}^{z} \frac{\partial}{\partial z}$, a representation of the Riemann surface $\mathcal{R}_{X} \backslash\{2$ half-logarithmic spirals of $X\}$ is given in (a). A deformed path that bounces off the half-logarithmic spiral determined by the branch cut is illustrated in (b). As a result, in (c) and (d) the deformed taut path $\widetilde{\Gamma}_{1} \cup \widetilde{\Gamma}_{2}$, recognizes the admissible word enclosed by the original $\gamma$ (or equivalently $\widetilde{\Gamma}$ ). See Example 9.2 .

Note that by definition the soul is a surface having $\widetilde{\Gamma}$ and the horizontal branch cuts $L_{j}=\left[a_{j}, \infty\right)_{+} \cup\left[a_{j}, \infty\right)_{-} \subset \mathcal{R}_{X}$, contained in its boundary.

Remark 9.3. In fact, if $X \in \mathcal{E}(d)$, it is clear that the soul of the $d$-configuration tree $\Lambda_{X}$ (see Definition 8.21) and the soul of the Riemann surface $\mathcal{R}_{X}$ agree up to a finite number of half-planes, which can be incorporated into the half-logarithmic spirals of $X$. This is again a reflection of the self-similar nature of the class 1 entire sectors $\mathscr{E}$ (as in the relation $E \mathscr{E} H \sim \mathscr{E}$, see (5.13) ).

Since each half-logarithmic spiral is homeomorphic to an open disk in $\mathcal{R}_{X}$ (see Figure 17. d for an example), we can assume under suitable deformation that; $\widetilde{\Gamma}$ is inside the soul of $\mathcal{R}_{X}$.

Consider the restriction to $\Gamma \subset \mathcal{R}_{X}$ of a height function

$$
\mathfrak{I m}\left(\Psi_{X}\right): \widetilde{\Gamma} \longrightarrow \mathbb{R} \text {. }
$$

After a small perturbation of the $\widetilde{\Gamma}$ in the soul of $\mathcal{R}_{X}$, the height function assumes only a finite number of local maxima and minima $\left\{p_{\alpha}\right\} \subset \widetilde{\Gamma}$, and a finite number of horizontal segments $\left\{\Sigma_{\beta}\right\} \subset \widetilde{\Gamma}$.

Let $p_{\varsigma} \in \widetilde{\Gamma}$ be a local maximum or minimum of the height function (9.1), it is an extreme point of $\widetilde{\Gamma}$ when in addition it belongs to a branch cut $L_{j}=\left[a_{j}, \infty\right)_{-} \cup$ $\left[a_{j}, \infty\right)_{+}$of the soul of $\mathcal{R}_{X}$. A bounded horizontal segment $\Sigma_{\varsigma} \subset \widetilde{\Gamma}$ is an extreme 
segment of $\widetilde{\Gamma}$ when it is contained in a branch cut $L_{j}$ of the soul of $\mathcal{R}_{X}$ and it is a maxima or minima of the height function (restricted to $\widetilde{\Gamma}$ as above).

Example 9.4. 1. In Figure 17, c, $\widetilde{\Gamma}_{1} \cup \widetilde{\Gamma}_{2}$ contains two extreme points. In Figure 18 (a), (b), (c) the respective $\widetilde{\Gamma}$ contain two extreme points. Moreover, (c) contains one extreme segment; (d) contains one extreme point and one extreme segment. In Figure 19, the arc $\widetilde{\Gamma}_{1}$ contains three extreme points, but the arc $\widetilde{\Gamma}_{3}$ does not contain any (certainly $\widetilde{\Gamma}_{3}$ intersects a branch cut, but these points are not maxima or minima for the height function).

2. Note that $\widetilde{\Gamma}_{1} \cup \widetilde{\Gamma}_{2}$ in Figure $17 \mathrm{c}-\mathrm{d}$ bounces off and assumes the minimum number of extreme points.

a)

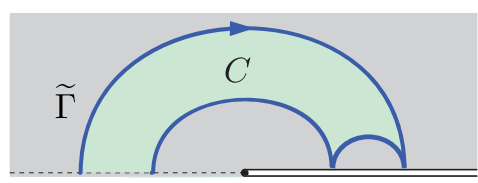

c)

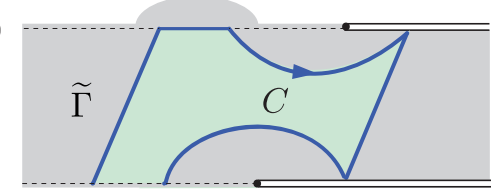

b)

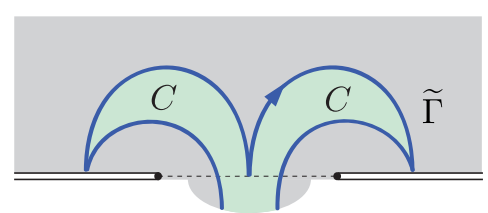

d)

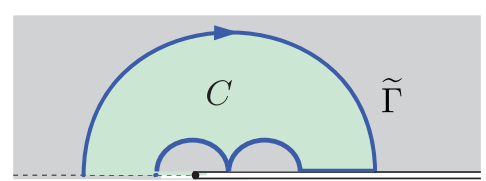

Figure 18. Four examples of $\widetilde{\Gamma} \subset \mathcal{R}_{X}$ containing extreme points and/or extreme segments. The arcs in (c) are in a finite height strip flow, the others are in half-planes. In all the cases a deformation of $\widetilde{\Gamma}$ allows a bounce off in exactly one point of each branch cut. Moreover, the extension $\mathcal{R}_{X} \cup C$ is obviously a Riemann surface. See Example 9.4 and Corollary 10.1 .

Step 2 (of proof of Theorem 9.1). In order to recognize sectors of type $\mathscr{E}$ we use the following.

Remark 9.5 (Bounce off and words aspects of a sheet of $\mathcal{R}_{X}$, for $((\mathbb{C}, 0), X)$ ). We recall Definition 8.3. Consider a sheet $\mathbb{C}_{t} \backslash\left\{L_{j}\right\}_{j=1}^{r}$ in the soul of $\mathcal{R}_{X}$ and assume that the sheet is glued to a half-logarithmic spiral along a branch cut $\left[a_{j}, \infty\right)_{ \pm}$ (resp. a left branch cut $\left.\left(\infty, a_{j}\right]_{ \pm}\right)$.

If $\widetilde{\Gamma}$ bounces off a branch cut $\left[a_{\mathrm{k}}, \infty\right)_{ \pm}$(resp. a left branch cut $\left.\left(\infty, a_{\mathrm{k}}\right]_{ \pm}\right)$, then $\widetilde{\Gamma}$ determines an entire sector $\mathscr{E}$.

Our goal is to perform a taut deformation of $\widetilde{\Gamma}$ in the soul of $\mathcal{R}_{X}$. This means the following.

i) The deformed $\widetilde{\Gamma}$ bounces off exactly once on each of the two boundaries $\left[a_{j}, \infty\right)_{ \pm}$arising from every branch cut $\left\{L_{j}\right\}_{j=1}^{d}$ in the soul of $\mathcal{R}_{X}$.

ii) The deformed $\widetilde{\Gamma}$ attains the minimum possible number of critical points of the height function $\mathfrak{I m}\left(\Psi_{X}\right)$ inside $\mathcal{R}_{X} \backslash\{2 d$ half-logarithmic spirals of $X\}$, see Figure 17 
iii) In addition, if not all the horizontal branch cuts lie on the same sheet (due to the existence of finite helicoids, recall Definition [5.4, in the soul of $\mathcal{R}_{X}$ ), then it might be necessary to keep deforming $\widetilde{\Gamma}$, so that it wraps around the finite helicoids in order to reach the sheets where the deformed $\widetilde{\Gamma}$ bounces off the (two) half-logarithmic spirals corresponding to each horizontal branch cut associated to each finite asymptotic value. When this happens, we will be able to recognize a sequence of elliptic $E_{\nu}$, or hyperbolic $H$, sectors. See Example 9.6 .

iv) It might be necessary to deform $\widetilde{\Gamma}$ across some of the finite height strip flows of the soul of $\mathcal{R}_{X}$. When this occurs we will be able to recognize parabolic sectors $P_{\nu}$ with $\nu$ corresponding to the displacement (the value of the integral of $\omega_{X}$ ) along the deformed $\gamma$ as it crosses the finite height strip flow.

The taut deformation of $\widetilde{\Gamma}$, can always be performed, since $\widetilde{\Gamma}$ is a generator of the fundamental group of $\mathcal{R}_{X} \backslash\{2 d$ half-logarithmic spirals $\}$.

By (anticlockwise respect to $\left(\mathbb{C}_{z}, 0\right)$ ) traveling around the taut deformed $\widetilde{\Gamma}$ we can read off the words associated to each possible sector: $E_{\nu}, H, P_{\nu}, \mathscr{E}_{\nu}$, according to their description given in 95 . In this way $\mathcal{W}_{X}$ is determined by the deformed $\gamma$ arising from the projection, under $\pi_{X, 1}$ in (2.6), of the taut deformation of $\widetilde{\Gamma}$.

This finishes the proof when all the $\left\{a_{j}\right\}$ are distinct and none share a horizontal and $\operatorname{Res}\left(\omega_{X}, 0\right)=0$.

Step 3 (of proof of Theorem 9.1). Consider now the case when two asymptotic values, say $a_{\sigma}$ and $a_{\rho}$, are on the same horizontal and there is a diagonal between them in $\mathcal{R}_{X}$. Recall that $\mathbb{C}_{t} \backslash L_{\sigma}$ are really copies of $\mathbb{C}_{t}$ cut along $L_{\sigma}$ but with the borders left there for further identification, then $\mathbb{C}_{t} \backslash\left(L_{\sigma} \cup L_{\rho}\right)$ presents no problem for the proof: having made the leftmost branch cut, there will be a choice of border (top or bottom) on which to make the other branch cut. The original $\widetilde{\Gamma}$ is in the soul of $\mathcal{R}_{X}$.

This leaves the case when some of the asymptotic values have multiplicity. Once again this poses no problem: there cannot be a diagonal between the preimages, $\pi_{X, 2}^{-1}\left(a_{j}\right)$, of an asymptotic value $a_{j}$ with multiplicity $\nu_{j}>1$.

Finally, if we assume that $\operatorname{Res}\left(\omega_{X}, 0\right) \neq 0$, then there exists a characteristic trajectory $\sigma$ of $\mathfrak{R e}(X)$ in $\bar{U}$ such that it starts at some point of $\gamma$ and has $\lim _{\tau \rightarrow \infty} \Psi_{X}(\sigma(\tau))=\infty$ (see footnote 6). We define $\mathcal{R}_{X}^{0}$ as the graph of $\Psi_{X}$ over $\bar{U} \backslash \sigma$. The boundary of $\mathcal{R}_{X}^{0}$ contains two copies of $\widetilde{\Sigma}=\Psi_{X}(\sigma)$ (as in Figure 9), we glue its boundary and get a new surface, say $\mathcal{R}_{X}$ (see Example 9.7). Note that, the fundamental group of $\mathcal{R}_{X}$ has the corresponding $\widetilde{\Gamma}$ as generator. Hence, all the above arguments can be repeated inside the soul of $\mathcal{R}_{X}$.

Theorem 9.1 is now exemplified for nontrivial objects: first in Example 9.6 the case when $\operatorname{Res}\left(\omega_{X}, 0\right)=0$ and secondly in Example 9.7 when $\operatorname{Res}\left(\omega_{X}, 0\right) \neq 0$.

Example 9.6 (Recognizing an admissible word on a Riemann surface with $d=4$ logarithmic branch points and $\left.\operatorname{Res}\left(\omega_{X}, 0\right)=0\right)$. In Figure 19 we have drawn the soul of a particular $\mathcal{R}_{X}$, together with the horizontal branch cuts (solid double lines) associated to the four distinct finite asymptotic values $\left\{a_{j}\right\}_{j=1}^{4}$. The four sheets shown are glued along the horizontal branch cuts (as in Corollary 5.11), according to the light blue dotted lines (all the glueings are made so that the solid dark blue path $\widetilde{\Gamma}_{1} \cup \cdots \cup \widetilde{\Gamma}_{6}$ is continuous). The segments $\overline{b_{1} b_{2}}, \overline{b_{1} b_{3}}$, and $\overline{b_{3} b_{4}}$ in the soul of 
$\mathcal{R}_{X}$ are the diagonals and appear as orange dotted lines (recall that $\pi_{X, 2}\left(b_{j}\right)=a_{j}$ ). However, none of the segments $\Delta_{\vartheta 14}, \Delta_{\vartheta 23}$, and $\Delta_{\vartheta 24}$ are diagonals. In order to complete the Riemann surface $\mathcal{R}_{X}$ one needs to glue to the soul a half-logarithmic spiral on each of the eight horizontal branch cuts that have not been identified.
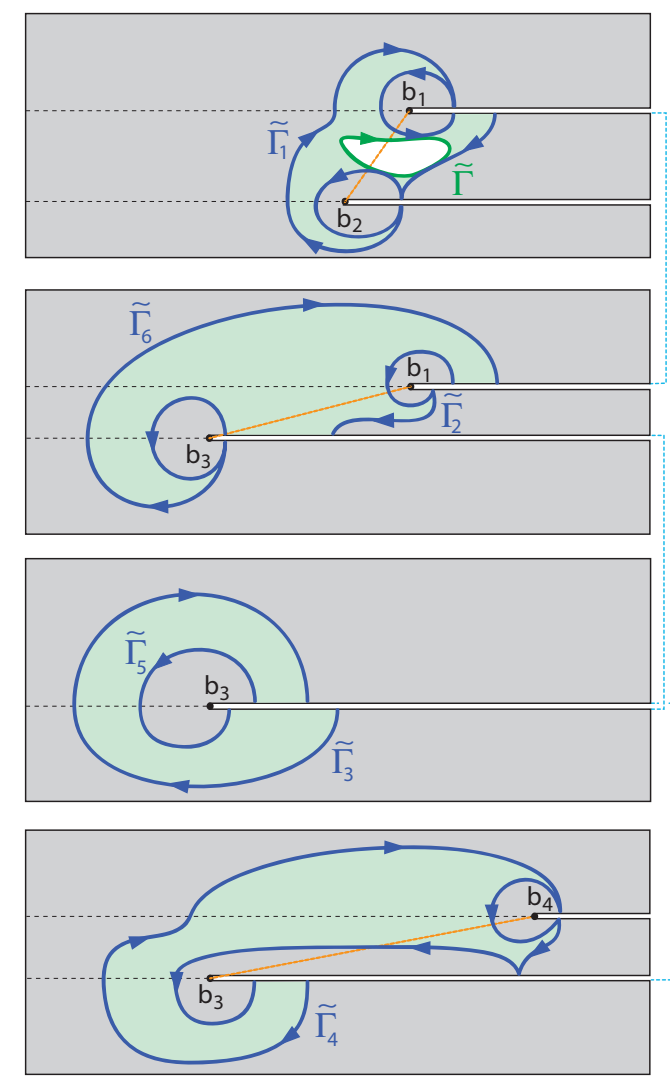

FiguRE 19. Soul of a Riemann surface $\mathcal{R}_{X}$ with $d=4$ logarithmic branch points and $\operatorname{Res}\left(\omega_{X}, 0\right)=0$. The green path $\widetilde{\Gamma}$ (corresponding to $\gamma)$ is deformed into the blue taut path $\widetilde{\Gamma}_{1} \cup \widetilde{\Gamma}_{2} \cup \cdots \cup \widetilde{\Gamma}_{6}$, which bounces off the corresponding branch cuts, in order to recognize the admissible word enclosed by $\gamma$. The exterior of $\widetilde{\Gamma}$ is colored white, while the exterior of the deformed $\widetilde{\Gamma}_{1} \cup \widetilde{\Gamma}_{2} \cup \cdots \cup \widetilde{\Gamma}_{6}$ is white and light green. In this case $X \in \mathcal{E}(4)$. See Example 9.6.

Note that the green path $\widetilde{\Gamma}$, corresponding to $\gamma$, lies completely on one sheet and that the interior of $\widetilde{\Gamma}$ encloses all the preimages (under $\pi_{X, 2}$ ) of the 4 finite asymptotic values. $\widetilde{\Gamma}$ does not define a cyclic word for the germ of the respective $X$. The path $\widetilde{\Gamma}$ is deformed into the taut path $\widetilde{\Gamma}_{1} \cup \widetilde{\Gamma}_{2} \cup \cdots \cup \widetilde{\Gamma}_{6}$. From it, we can now recognize the admissible word (we drop the sub-indices of the $P_{\nu}$ since they 
are not needed)

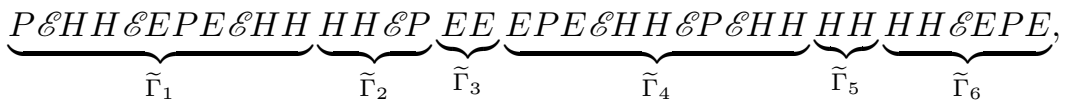

which in turn can be reduced (using (5.14) so that $H \mathscr{E} E \sim \mathscr{E}$ and $E \mathscr{E} H \sim \mathscr{E}$ ) to

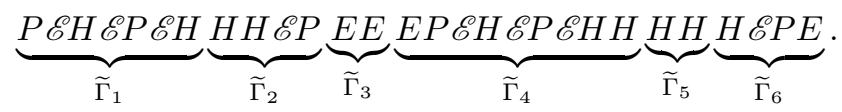

Example 9.7 (Recognizing an admissible word on a Riemann surface with $d=4$ logarithmic branch points and $\left.\operatorname{Res}\left(\omega_{Y}, 0\right) \neq 0\right)$. Consider once again Figure 19. but with the topmost sheet replaced with the one of Figure 20. This gives rise to the soul of Riemann surface $\mathcal{R}_{Y}$ as in the end of the proof of Theorem 9.1 .

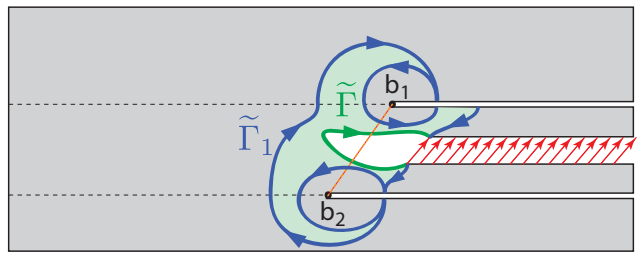

FiguRE 20. Soul of a Riemann surface $\mathcal{R}_{Y}$ with $d=4$ logarithmic branch points and $\operatorname{Res}\left(\omega_{Y}, 0\right) \neq 0$. Here we illustrate only the first sheet that replaces the topmost sheet shown in Figure 19, the rest remain the same. Once again, the green path $\widetilde{\Gamma}$ (corresponding to $\gamma)$ is deformed into the blue taut path $\widetilde{\Gamma}_{1} \cup \widetilde{\Gamma}_{2} \cup \cdots \cup \widetilde{\Gamma}_{6}$, which bounces off the corresponding branch cuts, in order to recognize the admissible word enclosed by $\gamma$. The exterior of $\widetilde{\Gamma}$ in $\mathcal{R}_{Y}$ is colored white, while the exterior of the deformed $\widetilde{\Gamma}_{1} \cup \widetilde{\Gamma}_{2} \cup \cdots \cup \widetilde{\Gamma}_{6}$ is white and light green. The nonzero residue corresponds to any of the red arrows (as in Figure 7), the red arrows also indicate that these boundaries should be identified to obtain $\mathcal{R}_{Y}$. See Example 9.7.

In this case, since $\operatorname{Res}\left(\omega_{Y}, 0\right) \neq 0$, the path $\widetilde{\Gamma}$ (corresponding to $\gamma$ ) is not closed. Hence the sheet containing $\widetilde{\Gamma}$ will have the right or left part of a finite height strip flow corresponding to the nonzero residue (see also Figure 7 for the analogous construction that corresponds to the residue). The boundaries of the added or missing part of the finite height strip flow can now be identified to obtain the soul of $\mathcal{R}_{Y}$. The admissible word can be read as before. In order to account for the residue, it is convenient to recall that letters $P_{\nu}$ in the admissible words have a sub-index $\nu_{j}$ indicating the displacement; see (5.6). Hence, once again taking into account the relations $H \mathscr{E} E \sim \mathscr{E}$ and $E \mathscr{E} H \sim \mathscr{E}$, we obtain the admissible word

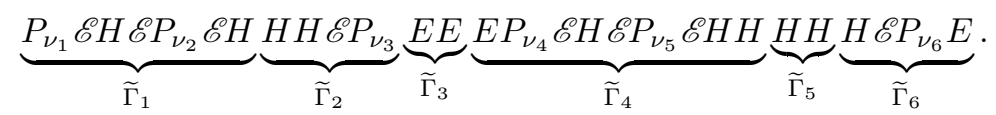

Note that each $\nu_{\iota} \in \mathbb{C}^{*}, \iota=1,2, \ldots, 6$ contributes to the residue; in the case at hand the contribution of $\nu_{3}=-\nu_{6}$, similarly $\nu_{4}=-\nu_{5}$, leaving the contributions of $\nu_{1}+\nu_{2}$ to be exactly the residue, i.e. $\operatorname{Res}\left(\omega_{Y}, 0\right)=\left(\nu_{1}+\nu_{2}\right) /(2 \pi i) \neq 0$. 
It is clear in Example 9.6 that since $\operatorname{Res}\left(\omega_{X}, \infty\right)=0, \mathcal{R}_{X}$ is the Riemann surface associated to some $X \in \mathcal{E}(4)$. On the other hand, Example 9.7 corresponds to an $Y \notin \mathcal{E}(4)$, because of Theorem 8.16 .

9.2. Germs from admissible words $\mathcal{W}_{X}$. With the results presented so far in \$5.9, particularly Proposition 5.20, and Theorem 9.1, we can now justify the term admissible for cyclic words comprised of the alphabet $H, E, P, \mathscr{E}$.

Recall that $\epsilon$ denotes the number of class 1 entire sectors $\mathscr{E}$ in an admissible word.

Theorem 9.8 (Recognition of analytical invariants from admissible words). Let $\mathcal{W}_{X}$ be an admissible word in the alphabet $H, E, P, \mathscr{E}$, with $\epsilon \geq 2$, coming from a germ $((\mathbb{C}, 0), X)$ of an isolated essential singularity of a complex analytic vector field. The distinguished parameter $\Psi_{X}$ satisfies that $\Psi_{X}^{-1}$ has $\epsilon / 2$ logarithmic branch points over $\epsilon / 2$ finite asymptotic values (up to translation) and $\epsilon / 2$ logarithmic branch points over $\infty$.

To illustrate the difficulties and the meaning of the assertion, we consider the following.

Example 9.9. Let $X(z)=i z \mathrm{e}^{z} \frac{\partial}{\partial z}$ be a singular analytic vector field on $\widehat{\mathbb{C}}$. There are singularities at 0 and $\infty$. Consider first the germ $((\widehat{\mathbb{C}}, \infty), X)$ and provide it with a simple path $\gamma$ enclosing $\infty \in \widehat{\mathbb{C}}$. It gives origin to the word

$$
\mathcal{W}_{X}=\mathscr{E} H H H \mathscr{E} E_{\nu},
$$

where $\nu=-2 \pi=\int_{\gamma} \omega_{X}$ is the complex time along $\gamma$ or displacement of $\mathcal{W}_{X}$. That is, the isometric glueing of $\mathscr{E}_{\nu}$ and $E$ requires a $-2 \pi$ surgery. Moreover, when we consider the germ $((\widehat{\mathbb{C}}, 0), X)$ provided with a simple path $-\gamma$ enclosing $0 \in \widehat{\mathbb{C}}$, it gives origin to the word

$$
\mathcal{W}_{X}=C_{i}
$$

where $-i=\operatorname{Res}\left(\omega_{X}, 0\right)$ and the displacement is $2 \pi=\int_{-\gamma} \omega_{X}$.

The global distinguished parameter

$$
\Psi_{X}(z)=\int_{z_{0}}^{z} \omega_{X}: \widehat{\mathbb{C}}_{z} \backslash\{\infty, 0\} \longrightarrow \mathbb{C}_{t},
$$

is multivalued.

A modification of the domain, will provide insight to the configuration of asymptotic values. We look at a punctured disk $D(\infty, r) \backslash\{\infty\}$ with the radius $r>0$ in the spherical metric, and cut the disk along $\mathbb{R}^{+} \cup\{\infty\}$. A univalued distinguished parameter is

$$
\Psi_{u n i, X}(z)=\int_{z_{0}}^{z} \omega_{X}: D(\infty, r) \backslash\left(\mathbb{R}^{+} \cup\{\infty\}\right) \longrightarrow \mathbb{C}_{t} .
$$

The inverse function $\Psi_{u n i, X}^{-1}$ has a logarithmic branch point over a finite asymptotic value $a \in \mathbb{C}_{t}$ and a logarithmic branch point over $\infty$. This is the assertion of Theorem 9.8 .

When gluing back the cut along $\left\{\mathbb{R}^{+} \cup \infty\right\}$ we obtain the multivalued $\Psi_{X} \mid D(\infty, r)$ that has an infinite configuration of asymptotic values $\{a+(-2 \pi) k \mid k \in \mathbb{Z}\}$, compare with Corollary 4.18 . 
Proof of Theorem 9.8. The admissible word $\mathcal{W}_{X}$ contains $\epsilon \geq 2$ class 1 entire sectors $\mathscr{E}$, whence 0 is an isolated essential singularity of $X$.

Furthermore, for the class 1 entire sectors that appear in $\mathcal{W}_{X}$, there is a natural pairing between letters $\infty_{\mathscr{E}_{f}}$ and ${ }_{f} \mathscr{E}_{\infty}$, recall (5.14), such that $\Psi_{\text {uni,X }}^{-1}$ has a logarithmic branch point over a finite asymptotic value.

In fact, class 1 entire sectors come in pairs; considering a word that contains class 1 entire sectors, say, for instance,

$$
\mathcal{W}_{X}=W_{1} \cdots \underbrace{\infty_{\mathscr{E}_{f}} H \cdots H_{f} \mathscr{E}_{\infty}}_{\text {no additional } \mathscr{E}} \cdots W_{n} .
$$

It is clear that both of the class 1 entire sectors will have the same finite asymptotic value associated to them. That is: the trajectories $\sigma_{1}, \sigma_{2}$ in $\infty_{\infty} \mathscr{E}_{f}$ and $f_{f} \mathscr{E}_{\infty}$ that arrive to the isolated essential singularity in finite time are asymptotic trajectories, and will be the ones that determine a finite asymptotic value (in particular these can also be characteristic trajectories).

Note that, $\Psi_{u n i, X}^{-1}$ has another logarithmic branch point over $\infty$ with multiplicity the number of pairs of class 1 entire sectors that appear in $\mathcal{W}_{X}$.

Thus,

$$
\Psi_{\text {uni }, X}(z)=\int_{z_{0}}^{z} \omega_{X}: D(\infty, r) \backslash\left\{\mathbb{R}^{+} \cup \infty\right\} \longrightarrow \mathbb{C}_{t}
$$

is a univalued function on this simply connected domain. $\Psi_{\text {uni, }}^{-1}$ has $d$ logarithmic branch points over the $d$ finite asymptotic values and $d$ logarithmic branch points over $\infty$, where $d=\epsilon / 2$ half the number of entire sectors in the admissible words.

This is the meaning of the assertion.

\section{A BetTer VeCtor Field GERM EXTENSION RESUlt}

Corollary 10.1 (Description of $X \in \mathcal{E}(d)$ as an admissible word). A germ $((\widehat{\mathbb{C}}, \infty)$, $X)$ is the restriction of an $X \in \mathcal{E}(d)$ if and only if $\infty \in \widehat{\mathbb{C}}$ is an isolated essential singularity and the admissible word $\mathcal{W}_{X}$ satisfies that

1) the residue of the word $\operatorname{Res}\left(\mathcal{W}_{X}\right)=0$,

2) the Poincaré-Hopf index of the word $\operatorname{PH}\left(\mathcal{W}_{X}\right)=2$,

3) it has exactly $\epsilon=2 d$ class 1 entire sectors $\mathscr{E}$.

Proof. $(\Rightarrow)(1)$ is obvious. (3) follows directly from Theorem 9.1

For $(2)$ consider that $X \in \mathcal{E}(d)$ is a nonvanishing analytic vector field on $\mathbb{C}$ with $\infty \in \widehat{\mathbb{C}}$ the unique singularity of $X$, then $\operatorname{PH}\left(\mathcal{W}_{X}\right)=P H(X, \infty)=\chi(\widehat{\mathbb{C}})=2$.

$(\Leftarrow)$ Considering the germ $((\widehat{\mathbb{C}}, \infty), X)$ and since $\operatorname{Res}(X, \infty)=\operatorname{Res}\left(\mathcal{W}_{X}\right)=0$ it follows that the distinguished parameter $\Psi_{X}:(\widehat{\mathbb{C}}, \infty) \rightarrow \mathbb{C}_{t}$ is univalued. From Theorem 9.8. $\Psi_{X}^{-1}$ has $\epsilon / 2$ logarithmic branch points over $\epsilon / 2$ finite asymptotic values and $\epsilon / 2$ logarithmic branch points over $\infty$.

Recall that $\mathcal{W}_{X}$ is an admissible word, hence there is a simple path $\gamma \subset\left(\widehat{\mathbb{C}}_{z}, \infty\right)$, enclosing $\infty$ once, that determines $\mathcal{W}_{X}$ (as in Theorem 9.1).

Notice that since $\operatorname{Res}(X, \infty)=0$, the path $\widetilde{\Gamma}(\tau)=\left(\gamma(\tau),\left(\Psi_{X} \circ \gamma\right)(\tau)\right) \subset \mathcal{R}_{X}$ is closed and because of Lemma 2.7, $\widetilde{\Gamma}$ has no self-intersections. Furthermore, $\widetilde{\Gamma}$ encloses the images, under $\pi_{X, 1}^{-1}$, of the angular sectors comprising $\mathcal{W}_{X}$ (as in Figures 17, 18 and 19, recall that the gray shaded area is the interior of $\widetilde{\Gamma})$. 
Step 1. Next, since our model of $\mathcal{R}_{X}$ is composed of copies of

$$
\mathbb{C}_{t} \backslash\{\text { suitable branch cuts }\} \quad \mathbb{C}_{t} \backslash(\{\text { suitable branch cuts }\} \cup\{C\}),
$$

where each $C$ is the missing region of $\mathbb{C}_{t}$ arising from the "exterior" of $\widetilde{\Gamma}$, we conclude that in fact $\partial C \subset \widetilde{\Gamma}$. See Figure 18 for examples. Each corresponding subset $C$ has at this moment indeterminate shape. We want to be able to recognize where the boundary of $C$ assumes a "bounce off like shape" (as in Figures 17 and 19), coming from the sectors $\mathscr{E} \in \mathcal{W}_{X}$. Using the local models for the angular sector $H, E, P, \mathscr{E}$ we can assume that $\widetilde{\Gamma}$ is a finite union of arcs of circle and horizontal segments (note that not necessarily all the critical points of horizontal segments of $\widetilde{\Gamma}$ are extreme).

In fact, consider a height function $\mathfrak{I m}\left(\Psi_{X}\right): \widetilde{\Gamma} \longrightarrow \mathbb{R}$; see (9.1).

Step 2. Let $p_{\varsigma}, \Sigma_{\varsigma}$ be an extreme point or an extreme segment of $\widetilde{\Gamma}$.

Since $p_{\varsigma}$ (resp. $\Sigma_{\varsigma}$ ) is a minima or maxima of the height function, an arc of $\widetilde{\Gamma}$ containing $p_{\varsigma}$ (resp. $\Sigma_{\varsigma}$ ) is contained in;

i) a half-plane $\overline{\mathbb{H}}^{2}$ in the soul of $\mathcal{R}_{X}$, or

ii) a finite height strip flow $S_{\nu}=\left\{\mathfrak{I m}\left(a_{j}\right) \leq \mathfrak{I m}(t) \leq \mathfrak{I m}\left(a_{k}\right)\right\}, \nu=a_{k}-a_{j}$, such that its two horizontal boundaries contain branch cuts (of asymptotic values $\left.a_{j}, a_{k}\right)$.

It is clear that $\overline{\mathbb{H}}^{2}$ (respectively $S_{\nu}$ ) in the soul of $\mathcal{R}_{X}$ is well defined. Hence, so is the missing region $C \subset \overline{\mathbb{H}}^{2}$ (respectively $S_{\nu}$ ); see Figure 18 .

Main Fact. By analytic extension of $\Psi_{X}^{-1}$, we can add to $\mathcal{R}_{X}$ the missing region $C$ as above, i.e. $\mathcal{R}_{X}$ can be extended to the Riemann surface, $\widetilde{\mathcal{R}}_{X}=\mathcal{R}_{X} \cup C$.

Let us consider some previous examples.

Example 10.2 (Examples 9.2 and 9.6 revisited). The extension is the inverse process in Figure 17. Considering the succession (c), (b), (a), both $\widetilde{\Gamma}_{1}$ and $\widetilde{\Gamma}_{2}$ contain one extreme point, the path in (b) contains one extreme point and one extreme segment, and finally in (a) by adding the topological disk bounded by $\widetilde{\Gamma}$, we obtain the desired $\widetilde{\mathcal{R}}_{X}$.

A slightly more complex example can be seen in Figure 19. starting with $\widetilde{\Gamma}=$ $\widetilde{\Gamma}_{1} \cup \cdots \cup \widetilde{\Gamma}_{6}$ we can deform $\widetilde{\Gamma}$ and add the corresponding $C$ 's to obtain half-planes and/or finite height strip flows. By doing this the original $\widetilde{\Gamma}$ is reduced to $\widetilde{\Gamma}_{0}$, and finally adding the topological disk bounded by $\widetilde{\Gamma}_{0}$, we obtain the desired $\widetilde{\mathcal{R}}_{X}$.

Note that other shapes for $C$ must be considered; see Figure 18 for some of them. However, in all cases the extension is well defined and $\mathcal{R}_{X} \cup C$ is a Riemann surface.

Step 3. In order to perform the construction of $\widetilde{\mathcal{R}}_{X}$, we proceed as follows:

i) For each extreme point $p_{\varsigma}$, or extreme segment $\Sigma_{\varsigma}$, we can add the missing exterior region $C_{\varsigma}$ in the respective half-plane or finite height strip flow containing a bounce off. We obtain an extended Riemann surface $\mathcal{R}_{X} \cup C_{\varsigma}$ with a new $\widetilde{\Gamma}_{\varsigma}$ as boundary. Recall that $\widetilde{\Gamma}_{\varsigma}$ has at least one new extreme segment.

ii) Continue until the extended Riemann surface has its boundary $\widetilde{\Gamma}_{-k}$, completely contained in one copy of $\mathbb{C}_{t}$. Moreover, note that $\widetilde{\Gamma}_{-k}$ bounds a topological disk in the copy of $\mathbb{C}_{t}$. Finally, extend the surface by adding the topological disk bounded by $\widetilde{\Gamma}_{-k}$, thus obtaining the desired $\widetilde{\mathcal{R}}_{X}$. 
This $\widetilde{\mathcal{R}}_{X}$ can be recognized (since $P H(X, \infty)=P H\left(\mathcal{W}_{X}\right)=2$ ) as the Riemann sphere $\widehat{\mathbb{C}}$ by the uniformization theorem.

Also, by Theorem 8.5 the extended Riemann surface $\widetilde{\mathcal{R}}_{X}$ is the Riemann surface of a vector field $\widetilde{X} \in \mathcal{E}(d)$.

Of course, this $\widetilde{X}$ has the original germ as the singularity at $\infty$.

Note that the second part of the proof of the previous corollary can be interpreted as an extension result.

Corollary 10.3. Let $((\mathbb{C}, 0), X)$ be a germ of singular analytic vector field defining an admissible word $\mathcal{W}_{X}$ in $H, E, P, \mathscr{E}$, with $\operatorname{Res}\left(\omega_{X}, 0\right)=0$, and assume that the Poincaré-Hopf index $P H(X, 0)=2-2 \mathfrak{g}$ for $\mathfrak{g} \in \mathbb{N} \cup\{0\}$ a nonnegative integer. Then there exists an extended singular analytic vector field $\widetilde{X}$ on the compact Riemann surface $M_{\mathfrak{g}}$, of genus $\mathfrak{g}$, such that: $\widetilde{X}$ has the original germ $((\mathbb{C}, 0), X)$ as a singularity and

a) no other singularities on $M_{0} \cong \widehat{\mathbb{C}}$, when $\mathfrak{g}=0$,

b) two new simple poles and no other singularities on $M_{\mathfrak{g}}$, when $\mathfrak{g} \geq 1$.

Proof. As mentioned, the proof of Corollary 10.1 proves the case $M_{0} \cong \widehat{\mathbb{C}}$.

For the general case perform surgery, as in Step 2 of the proof of Theorem 7.1 . adding two simple poles and no other singularities.

\section{Appendix: Global decomposition into Canonical Regions, FOLlOWING L. MARKus AND H. E. BENZINGER}

According to L. Markus [47, see also [55, 21] pp. 33, the

- spiral $\left(\mathbb{R}^{2} \backslash\{0\}, x \frac{\partial}{\partial x}+y \frac{\partial}{\partial y}\right)$,

- annulus $\left(\mathbb{R}^{2} \backslash\{0\},-y \frac{\partial}{\partial x}+x \frac{\partial}{\partial y}\right)$ and

- $\operatorname{strip}\left(\mathbb{R}^{2}, \frac{\partial}{\partial x}\right)$

are the real models for the search of a topological decomposition of $C^{1}$ phase portraits of real vector fields $Z$ on a region $U \subset \mathbb{R}^{2}$. These models are sometimes denoted canonical regions. Philosophically, canonical regions are open connected subsets $V \subset U$, invariant under the flow of $Z$, where $Z$ is parallel and maximal respect to the above properties.

From our point of view, canonical regions $V^{\prime} \subset U$ are the largest domains where $Z$ admits a $C^{1}$ "flow box" having maximal domain $\Psi_{Z}$; i.e. $\Psi_{Z}$ sends $\left(V^{\prime}, Z\right)$ to a spiral, annulus or strip.

The list of canonical regions of L. Markus can be enriched in the complex analytic category, as in Table 3, where for a singular complex analytic vector field $(M, X)$ we consider $\mathfrak{R e}(X)$ on $M$.

With this in mind, a result of H. E. Benzinger (see [10], pp. 466) can be interpreted as follows.

Theorem 11.1 (H. E. Benzinger). A rational vector field $X$ on $\widehat{\mathbb{C}}$ admits a finite decomposition in strip flows, annulus flows and spiral flows.

Proof. Note that if $X$ admits a spiral flow, an infinite height strip flow, or an infinite height annulus flow, then $X$ is holomorphic on $\widehat{\mathbb{C}}$.

Assume that $X$ has at least one pole. A maximal trajectory $\left\{z_{j}(\tau):\left(\tau_{\min }, \tau_{\max }\right)\right.$ $\left.\subset \mathbb{R} \rightarrow \widehat{\mathbb{C}}_{z}\right\}$ of $X$ is a separatrix if its domain is a strict subset of $\mathbb{R}$. Equivalently, 
TABle 3. Complex analytic canonical regions of $X$ on $M$.

\begin{tabular}{|c|c|c|}
\hline dynamics & geometry & nomenclature \\
\hline \multicolumn{3}{|c|}{ spiral flow } \\
\hline$\left(\mathbb{C}^{*}, \lambda z \frac{\partial}{\partial z}\right)$ & $\mathbb{C}^{*}$ & spiral flow $\lambda \in \mathbb{C} \backslash i \mathbb{R}$ \\
\hline \multicolumn{3}{|c|}{ annulus flow $\left(i \lambda_{2} \in i \mathbb{R}^{*}\right)$} \\
\hline $\begin{array}{c}\left(\mathbb{C}^{*}, i \lambda_{2} \frac{\partial}{\partial z}\right) \\
\left(\{0<|z|<1\}, i \lambda_{2} \frac{\partial}{\partial z}\right) \\
\left(\{1<|z|<h\}, i \lambda_{2} \frac{\partial}{\partial z}\right)\end{array}$ & $\begin{array}{c}\mathbb{C}^{*} \\
D(0,1) \backslash\{0\} \\
\{1<|z|<h\}\end{array}$ & $\begin{array}{c}\text { infinite height } \\
\text { annulus flow (cylinder), } \\
\text { semi-infinite height } \\
\text { annulus flow (cylinder), } \\
\text { finite height } \\
\text { annulus flow (cylinder), }\end{array}$ \\
\hline \multicolumn{3}{|c|}{ strip flow } \\
\hline $\begin{array}{c}\left(\mathbb{C}, \frac{\partial}{\partial z}\right) \\
\left(\overline{\mathbb{H}}_{ \pm}^{2}, \frac{\partial}{\partial z}\right) \\
\left(\{0<\mathfrak{I m}(z)<h\}, \frac{\partial}{\partial z}\right)\end{array}$ & $\begin{array}{c}\mathbb{C} \\
\overline{\mathbb{H}}^{2} \\
\{0<\mathfrak{I m}(z)<h\}\end{array}$ & $\begin{array}{c}\text { infinite height } \\
\text { strip flow (plane) } \\
\text { semi-infinite height } \\
\text { strip flow (half-plane) } \\
\text { finite height strip flow }\end{array}$ \\
\hline \multicolumn{3}{|c|}{$\begin{array}{c}\text { union of } \\
\text { semi-infinite height strip flows }\end{array}$} \\
\hline $\begin{array}{c}\left(\overline{\mathbb{H}}_{ \pm}^{2}, \mathrm{e}^{z} \frac{\partial}{\partial z}\right) \\
\left(\{0<|\mathfrak{I m}(z)|<2 K \pi\}, \mathrm{e}^{z} \frac{\partial}{\partial z}\right)\end{array}$ & $\begin{array}{l}\overline{\mathbb{H}}_{ \pm}^{2} \cup \overline{\mathbb{H}}_{\mp}^{2} \cup \ldots \\
\overline{\mathbb{H}}_{ \pm}^{2} \cup \ldots \cup \overline{\mathbb{H}}_{ \pm}^{2}\end{array}$ & $\begin{array}{l}\text { half-logarithmic spiral } \\
\text { (semi-infinite helicoid) } \\
\text { finite helicoid } \\
\end{array}$ \\
\hline
\end{tabular}

the image of $z_{j}(\tau)$ accumulates at one pole; its flow is not analytic in at least one direction. Removing from $\widehat{\mathbb{C}}_{z}$ the zeros, the poles of $X$ and its separatrices we get a finite number of open sets $\left\{U_{\beta}\right\} \subset \widehat{\mathbb{C}}_{z}$ that are the desired strip flows, annulus flows and/or spiral flows.

Remark 11.2. Let $X$ be a singular analytic vector field on $M$, having singularities determining admissible words $\mathcal{W}_{X}$. There exist a one-to-one correspondence between:

- angular sectors $\mathscr{E}$ and

- semi-infinite helicoids (or equivalently half-logarithmic spirals) $\overline{\mathbb{H}}_{ \pm}^{2} \cup \overline{\mathbb{H}}_{\mp}^{2} \cup \ldots$.

Finally, by considering the singular analytic category with one isolated essential singularity, and introducing (for convenience of the reader) the two models based on the semi-infinite helicoid and the finite helicoid, we have:

Corollary 11.3 ( Global decomposition of meromorphic on $\mathbb{C}$, class 1 vector fields). $A$ vector field $X$, meromorphic on $\mathbb{C}$, with an isolated essential singularity at $\infty \in$ $\widehat{\mathbb{C}}$, with $\Psi_{X}^{-1}$ having d logarithmic branch points over $d$ finite asymptotic values and d logarithmic branch points over $\infty$, admits a finite decomposition in $2 d$ halflogarithmic spirals, semi-infinite height strip flows, finite height strip flows, annulus flows and spiral flows. 
Proof. Follows immediately from Benzinger's result (Theorem 11.1), the local realization (Theorem 9.1) and the recognition of analytical invariants from admissible words (Theorem 9.8).

Example 11.4. Every vector field $X \in \mathcal{E}(d)$ admits a finite decomposition as in the corollary (without annuli or spirals).

As a particular example, consider Figure 15, corresponding to $X(z)=\mathrm{e}^{i \frac{\pi}{4}} \mathrm{e}^{z^{2}} \frac{\partial}{\partial z}$; one can observe the appearance of a finite height strip flow and 4 half-logarithmic spirals.

In Examples 11.5 and 11.6, together with their corresponding figures, we can observe some of the decomposition pieces for the 1-order 3 meromorphic case. For instance in Figure 21, b finite height strip flows can be observed between the poles, in both Figures 21 a and 21, we can observe 6 half-logarithmic spirals.
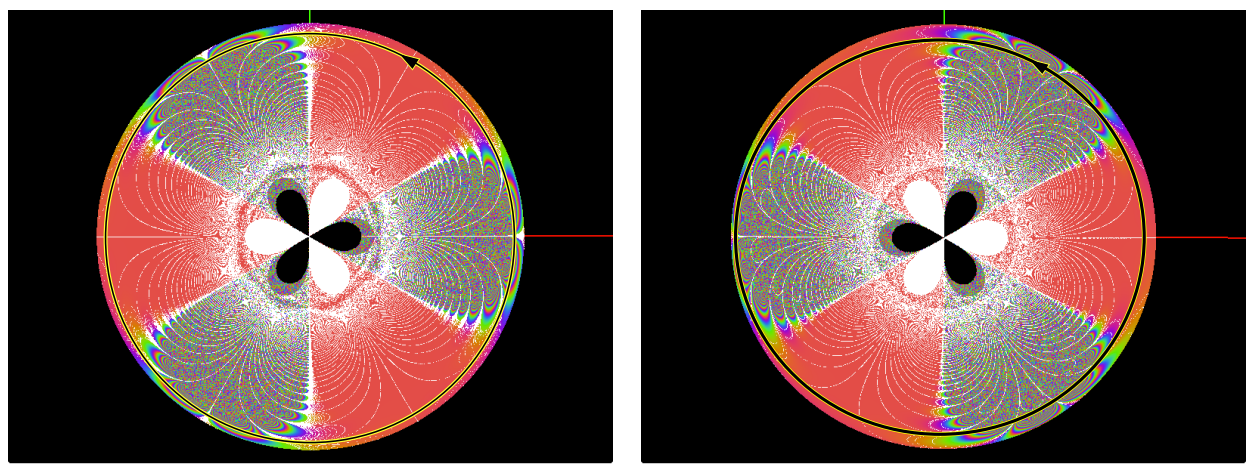

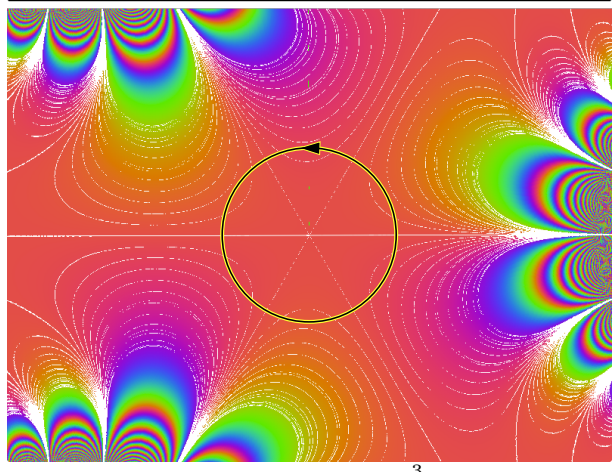

(a) $X(z)=-\frac{\mathrm{e}^{z^{3}}}{3 z^{2}} \frac{\partial}{\partial z}$

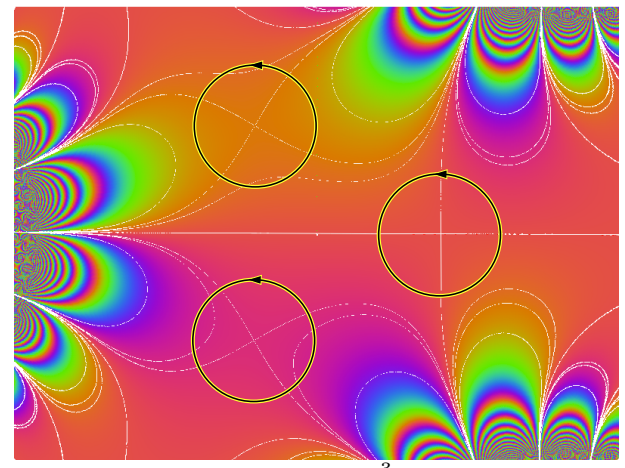

(b) $X(z)=\frac{\mathrm{e}^{z^{3}}}{3 z^{3}-1} \frac{\partial}{\partial z}$

Figure 21. Phase portraits of the vector fields of Examples 11.5 and 11.6 as seen from two different perspectives: in the vicinity of $\infty \in \widehat{\mathbb{C}}_{z}$ and on the plane near the origin. The black and green paths around the singularities, corresponding to the respective $\{\gamma\}$, are added to illustrate the different sectors that appear at each singularity.

Example 11.5. Consider the vector field $X(z)=-\frac{\mathrm{e}^{z^{3}}}{3 z^{2}} \frac{\partial}{\partial z}$; see Figure 21 (a). In this case $X$ has an isolated essential singularity at $\infty \in \widehat{\mathbb{C}}$ and a double pole at the 
origin. Furthermore, there are 6 entire $\mathscr{E}$ sectors in the vicinity of infinity, so the admissible word at $\infty$ is

\section{$E \mathscr{E} H H \mathscr{E} E E \mathscr{E} H H \mathscr{E} E E \mathscr{E} H H \mathscr{E} E$,}

hence the Poincaré-Hopf index of $X$ at $\infty$ is 4 , while the admissible word at 0 is $\mathrm{HHHHH}$, and its Poincaré-Hopf index is -2 , and of course

$$
\chi(\widehat{\mathbb{C}})=P H(X, \infty)+P H(X, 0)=4-2 .
$$

Example 11.6 (Example4.17 revisited). Consider the vector field $X(z)=\frac{\mathrm{e}^{z^{3}}}{3 z^{3}-1} \frac{\partial}{\partial z}$; see Figure 21 (b). Once again $\infty$ is an isolated essential singularity of $X$, but now $X$ has 3 simple poles located at $\frac{1}{\sqrt[3]{3}}, \frac{\mathrm{e}^{i 2 \pi / 3}}{\sqrt[3]{3}}$ and $\frac{\mathrm{e}^{-i 2 \pi / 3}}{\sqrt[3]{3}}$. The admissible word at $\infty$ is (starting from the top in Figure 21 (b)):

\section{$\mathscr{E} H H \mathscr{E} E E \mathscr{E} H H \mathscr{E} E E E \mathscr{E} H H \mathscr{E} E E E$,}

so the Poincaré-Hopf index of $X$ at $\infty$ is 5 , while the admissible word at each simple pole is $H H H H$, and the corresponding Poincaré-Hopf index is -1, and of course once again

$\chi(\widehat{\mathbb{C}})=P H(X, \infty)+P H\left(X, \frac{1}{\sqrt[3]{3}}\right)+P H\left(X, \frac{\mathrm{e}^{i 2 \pi / 3}}{\sqrt[3]{3}}\right)+P H\left(X, \frac{\mathrm{e}^{-i 2 \pi / 3}}{\sqrt[3]{3}}\right)=2$.

\section{REFERENCES}

[1] L. V. Ahlfors, Conformal invariants: topics in geometric function theory, McGraw-Hill Book Co., New York-Düsseldorf-Johannesburg, 1973. McGraw-Hill Series in Higher Mathematics. MR.0357743

[2] L. V. Ahlfors, Complex analysis: An introduction to the theory of analytic functions of one complex variable; International Series in Pure and Applied Mathematics, 3rd ed., McGrawHill Book Co., New York, 1978. MR.510197

[3] A. Alvarez-Parrilla, Complex analytic vector field visualization without numerical integration, Preprint (2009).

[4] A. Alvarez-Parrilla, A. Gómez-Arciga, and A. Riesgo-Tirado, Newton vector fields on the plane and on the torus, Complex Var. Elliptic Equ. 54 (2009), no. 5, 449-461, DOI 10.1080/17476930902755658. MR2524140

[5] A. Alvarez-Parrilla, M. E. Frías-Armenta, E. López-González, and C. Yee-Romero, On solving systems of autonomous ordinary differential equations by reduction to a variable of an algebra, Int. J. Math. Math. Sci. (2012), Art. ID 753916, 21. MR2974702

[6] A. A. Andronov, E. A. Leontovich, I. I. Gordon, and A. G. Maľer, Qualitative theory of second-order dynamic systems, Halsted Press (A division of John Wiley \& Sons), New YorkToronto, Ont.; Israel Program for Scientific Translations, Jerusalem-London, 1973. Translated from the Russian by D. Louvish. MR0350126

[7] E. Arbarello, M. Cornalba, and P. A. Griffiths, Geometry of algebraic curves. Volume II, Grundlehren der Mathematischen Wissenschaften [Fundamental Principles of Mathematical Sciences], vol. 268, Springer, Heidelberg, 2011. With a contribution by Joseph Daniel Harris. MR.2807457

[8] L. Arnold, C. Jones, K. Mischaikow, and G. Raugel, Dynamical systems, Lecture Notes in Mathematics, vol. 1609, Springer-Verlag, Berlin, 1995. Lectures given at the Second C.I.M.E. Session held in Montecatini Terme, June 13-22, 1994; Edited by R. Johnson. MR1374106

[9] G. V. Belyŭ, Galois extensions of a maximal cyclotomic field (Russian), Izv. Akad. Nauk SSSR Ser. Mat. 43 (1979), no. 2, 267-276, 479. MR.534593

[10] H. E. Benzinger, Plane autonomous systems with rational vector fields, Trans. Amer. Math. Soc. 326 (1991), no. 2, 465-483, DOI 10.2307/2001769. MR992604

[11] C. A. Berenstein and R. Gay, Complex variables: An introduction, Graduate Texts in Mathematics, vol. 125, Springer-Verlag, New York, 1991. MR.1107514 
[12] W. Bergweiler and A. Eremenko, On the singularities of the inverse to a meromorphic function of finite order, Rev. Mat. Iberoamericana 11 (1995), no. 2, 355-373, DOI 10.4171/RMI/176. MR 1344897

[13] B. Branner and K. Dias, Classification of complex polynomial vector fields in one complex variable, J. Difference Equ. Appl. 16 (2010), no. 5-6, 463-517, DOI 10.1080/10236190903251746. MR2642463

[14] L. Brickman and E. S. Thomas, Conformal equivalence of analytic flows, J. Differential Equations 25 (1977), no. 3, 310-324, DOI 10.1016/0022-0396(77)90047-X. MR0447674

[15] J. P. Bowman and F. Valdez, Wild singularities of flat surfaces, Israel J. Math. 197 (2013), no. 1, 69-97, DOI 10.1007/s11856-013-0022-y. MR3096607

[16] X. Buff and L. Tan, Dynamical convergence and polynomial vector fields, J. Differential Geom. 77 (2007), no. 1, 1-41. MR2344353

[17] A. Bustinduy, L. Giraldo, and J. Muciño-Raymundo, Jacobian mates for non-singular polynomial maps in $\mathbb{C}^{n}$ with one-dimensional fibers, J. Singul. 9 (2014), 27-42. MR 3249045

[18] A. Bustinduy, L. Giraldo, and J. Muciño-Raymundo, Vector fields from locally invertible polynomial maps in $\mathbb{C}^{n}$, Colloq. Math. 140 (2015), no. 2, 205-220, DOI 10.4064/cm140-2-4. MR 3371776

[19] A. Douady, F. Estrada, P. Sentenac, Champs de vecteurs polynomiaux sur $\mathbb{C}$, Preprint 2005.

[20] J. J. Duistermaat and J. A. C. Kolk, Lie groups, Universitext, Springer-Verlag, Berlin, 2000. MR.1738431

[21] F. Dumortier, J. Llibre, and J. C. Artés, Qualitative theory of planar differential systems, Universitext, Springer-Verlag, Berlin, 2006. MR2256001

[22] G. Elfving, Über eine Klasse von Riemannschen Flächen und ihre Uniformisierung, Acta Soc. Sci. Fennicae, N.S. 2, Nr. 3 (1934), 1-60.

[23] M.-E. Frías-Armenta and J. Muciño-Raymundo, Topological and analytical classification of vector fields with only isochronous centres, J. Difference Equ. Appl. 19 (2013), no. 10, 16941728, DOI 10.1080/10236198.2013.772598. MR3173513

[24] A. Garijo, A. Gasull, and X. Jarque, Normal forms for singularities of one dimensional holomorphic vector fields, Electron. J. Differential Equations (2004), No. 122, 7. MR2108893

[25] A. Garijo, A. Gasull, and X. Jarque, Local and global phase portrait of equation $\dot{z}=f(z)$, Discrete Contin. Dyn. Syst. 17 (2007), no. 2, 309-329. MR2257435

[26] J. Gregor, Dynamické systémy s regulární pravou stranou I, Pokroky Mat. Fyz. Astron. 3 (1958), 153-160.

[27] J. Gregor, Dynamické systémy s regulární pravou stranou II, Pokroky Mat. Fyz. Astron. 3 (1958), 266-270.

[28] P. Griffiths and J. Harris, Principles of algebraic geometry, Wiley-Interscience [John Wiley \& Sons], New York, 1978. Pure and Applied Mathematics. MR.507725

[29] W. Groß, Über die Singularitäten analytischer Funktionen (German), Monatsh. Math. Phys. 29 (1918), no. 1, 3-47, DOI 10.1007/BF01700480. MR.1548975

[30] C. Gutiérrez, Smoothing continuous flows on two-manifolds and recurrences, Ergodic Theory Dynam. Systems 6 (1986), no. 1, 17-44, DOI 10.1017/S0143385700003278. MR837974

[31] O. Hájek, Notes on meromorphic dynamical systems. I (English, with Russian summary), Czechoslovak Math. J. 16 (91) (1966), 14-27. MR.0194661

[32] O. Hájek, Notes on meromorphic dynamical systems. II (English, with Russian summary), Czechoslovak Math. J. 16 (91) (1966), 28-35. MR0194662

[33] O. Hájek, Notes on meromorphic dynamical systems. III (English, with Russian summary), Czechoslovak Math. J. 16 (91) (1966), 36-40. MR.0194663

[34] K. Hockett and S. Ramamurti, Dynamics near the essential singularity of a class of entire vector fields, Trans. Amer. Math. Soc. 345 (1994), no. 2, 693-703, DOI 10.2307/2154994. MR 1270665

[35] X.-H. Hua and C.-C. Yang, Dynamics of transcendental functions, Asian Mathematics Series, vol. 1, Gordon and Breach Science Publishers, Amsterdam, 1998. MR1652248

[36] A. Hurwitz, Sur les points critiques des fonctions inverses, Comptes Rendus 143 (1906), 877-879; Math. Werke, Bd. 1, S. 655-656.

[37] Y. Ilyashenko and S. Yakovenko, Lectures on analytic differential equations, Graduate Studies in Mathematics, vol. 86, American Mathematical Society, Providence, RI, 2008. MR2363178

[38] F. Iversen, Recherches sur les fonctions inverses des fonctions méromorphes, Thèse, Helsingfors, 1914. 
[39] J. A. Jenkins, Univalent functions and conformal mapping, Ergebnisse der Mathematik und ihrer Grenzgebiete. Neue Folge, Heft 18. Reihe: Moderne Funktionentheorie, Springer-Verlag, Berlin-Göttingen-Heidelberg, 1958. MR0096806

[40] H. Th. Jongen, P. Jonker, and F. Twilt, On the classification of plane graphs representing structurally stable rational Newton flows, J. Combin. Theory Ser. B 51 (1991), no. 2, 256-270, DOI 10.1016/0095-8956(91)90041-H. MR1099075

[41] S. Kobayashi, Transformation groups in differential geometry, Springer-Verlag, New YorkHeidelberg, 1972. Ergebnisse der Mathematik und ihrer Grenzgebiete, Band 70. MR0355886

[42] S. Kobayashi, K. Nomizu, Foundations of Differential Geometry Vol. 2., John Wiley \& Sons, New York, 1969.

[43] F. Klein, On Riemann's Theory of Algebraic Functions and Their Integrals, Dover Publications Inc., New York, 1963.

[44] M. Kontsevich and A. Zorich, Connected components of the moduli spaces of Abelian differentials with prescribed singularities, Invent. Math. 153 (2003), no. 3, 631-678, DOI 10.1007/s00222-003-0303-x. MR2000471

[45] J. Muciño-Raymundo, Complex structures adapted to smooth vector fields, Math. Ann. 322 (2002), no. 2, 229-265, DOI 10.1007/s002080100206. MR1893915

[46] N. A. Lukashevich, Isochronicity of a center for certain systems of differential equations, Differ. Uravn. 1 (1965), 295-302.

[47] L. Markus, Global structure of ordinary differential equations in the plane, Trans. Amer. Math. Soc. 76 (1954), 127-148, DOI 10.2307/1990747. MR.0060657

[48] H. Masur and S. Tabachnikov, Rational billiards and flat structures, Handbook of dynamical systems, Vol. 1A, North-Holland, Amsterdam, 2002, pp. 1015-1089, DOI 10.1016/S1874575X(02)80015-7. MR 1928530

[49] J. Muciño-Raymundo, Complex structures adapted to smooth vector fields, Math. Ann. 322 (2002), no. 2, 229-265, DOI 10.1007/s002080100206. MR1893915

[50] J. Muciño-Raymundo and C. Valero-Valdés, Bifurcations of meromorphic vector fields on the Riemann sphere, Ergodic Theory Dynam. Systems 15 (1995), no. 6, 1211-1222, DOI 10.1017/S0143385700009883. MR1366317

[51] D. Mumford, Curves and their Jacobians, The University of Michigan Press, Ann Arbor, Mich., 1975. MR0419430

[52] D. J. Needham and A. C. King, On meromorphic complex differential equations, Dynam. Stability Systems 9 (1994), no. 2, 99-122, DOI 10.1080/02681119408806171. MR 1287510

[53] R. Nevanlinna, Analytic functions, Translated from the second German edition by Phillip Emig. Die Grundlehren der mathematischen Wissenschaften, Band 162, Springer-Verlag, New York-Berlin, 1970. MR0279280

[54] R. Nevanlinna, Über Riemannsche Flächen mit endlich vielen Windungspunkten (German), Acta Math. 58 (1932), no. 1, 295-373, DOI 10.1007/BF02547780. MR1555350

[55] D. A. Neumann, Classification of continuous flows on 2-manifolds, Proc. Amer. Math. Soc. 48 (1975), 73-81, DOI 10.2307/2040695. MR0356138

[56] F. W. J. Olver, Asymptotics and special functions, AKP Classics, A K Peters, Ltd., Wellesley, MA, 1997. Reprint of the 1974 original [Academic Press, New York; MR0435697 (55 \#8655)]. MR 1429619

[57] R. Peretz, Maximal domains for entire functions, J. Anal. Math. 61 (1993), 1-28, DOI 10.1007/BF02788836. MR1253436

[58] B. Riemann, Collected papers, Kendrick Press, Heber City, UT, 2004. Translated from the 1892 German edition by Roger Baker, Charles Christenson and Henry Orde. MR 2121437

[59] M. Sabatini, Characterizing isochronous centres by Lie brackets, Differential Equations Dynam. Systems 5 (1997), no. 1, 91-99. MR1656001

[60] S. L. Segal, Nine introductions in complex analysis, Revised edition, North-Holland Mathematics Studies, vol. 208, Elsevier Science B.V., Amsterdam, 2008. MR2376066

[61] M. Shub, D. Tischler, and R. F. Williams, The Newtonian graph of a complex polynomial, SIAM J. Math. Anal. 19 (1988), no. 1, 246-256, DOI 10.1137/0519018. MR924558

[62] S. Smale, A convergent process of price adjustment and global Newton methods, J. Math. Econom. 3 (1976), no. 2, 107-120, DOI 10.1016/0304-4068(76)90019-7. MR0411577

[63] S. Smale, The fundamental theorem of algebra and complexity theory, Bull. Amer. Math. Soc. (N.S.) 4 (1981), no. 1, 1-36, DOI 10.1090/S0273-0979-1981-14858-8. MR590817 
[64] A. Speiser, Untersuchungen über konforme und quasikonforme Abbildung, Dtsch. Math. 3 (1938).

[65] A. Speiser, Ueber Riemannsche Flächen (German), Comment. Math. Helv. 2 (1930), no. 1, 284-293, DOI 10.1007/BF01214465. MR.1509419

[66] K. Strebel, Quadratic differentials, Ergebnisse der Mathematik und ihrer Grenzgebiete (3) [Results in Mathematics and Related Areas (3)], vol. 5, Springer-Verlag, Berlin, 1984. MR743423

[67] M. Taniguchi, Explicit representation of structurally finite entire functions, Proc. Japan Acad. Ser. A Math. Sci. 77 (2001), no. 4, 68-70. MR.1829377

[68] M. Taniguchi, Synthetic deformation space of an entire function, Value distribution theory and complex dynamics (Hong Kong, 2000), Contemp. Math., vol. 303, Amer. Math. Soc., Providence, RI, 2002, pp. 107-136, DOI 10.1090/conm/303/05238. MR.1943529

[69] W. P. Thurston, Three-dimensional geometry and topology. Vol. 1, Princeton Mathematical Series, vol. 35, Princeton University Press, Princeton, NJ, 1997. Edited by Silvio Levy. MR.1435975

[70] E. P. Volokitin and V. V. Ivanov, Isochronicity and commutability of polynomial vector fields (Russian, with Russian summary), Sibirsk. Mat. Zh. 40 (1999), no. 1, 30-48, i, DOI 10.1007/BF02674287; English transl., Siberian Math. J. 40 (1999), no. 1, 23-38. MR.1686982

[71] H. H. Wu, Function theory on noncompact Kähler manifolds, Complex differential geometry, DMV Sem., vol. 3, Birkhäuser, Basel, 1983, pp. 67-155, DOI 10.1007/978-3-0348-6566-1_2. MR.826253

Grupo Alximia SA de CV, Ensenada, Baja California, México

E-mail address: alvaro.uabc@gmail.com

Centro de Ciencias Matemáticas, Universidad Nacional Autónoma de México

E-mail address: muciray@matmor.unam.mx 